Copyright:The author, Sara Hamis, 2021.

\title{
Multiscale Modelling and Analysis of Tumour Growth and Treatment Strategies
}

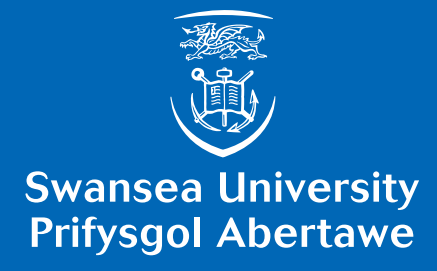

\author{
Sara Hamis \\ Department of Mathematics \\ Swansea University
}

Submitted to Swansea University in fulfilment of the requirements for the degree of

Doctor of Philosophy 



\begin{abstract}
A multiscale, agent-based mathematical framework is here used to capture the multiscale nature of solid tumours. Tumour dynamics and treatment responses are modelled and simulated in silico. Details regarding cell cycle progression, tumour growth and oxygen distribution are included in the mathematical framework. Treatment responses to conventional anti-cancer therapies, such as chemotherapy and radiotherapy, as well as to more novel drugs, such as hypoxia-activated prodrugs and DNA-damage repair inhibiting drugs, are studied. Uncertainty and sensitivity analyses techniques are discussed in order to mitigate model uncertainty and interpret model sensitivity to parameter perturbations. This thesis furthermore discusses the role of mathematical modelling in current cancer research.
\end{abstract}




\section{Declaration}

\section{Statement 1}

This work has not previously been accepted in substance for any degree and is not being concurrently submitted in candidature for any degree.

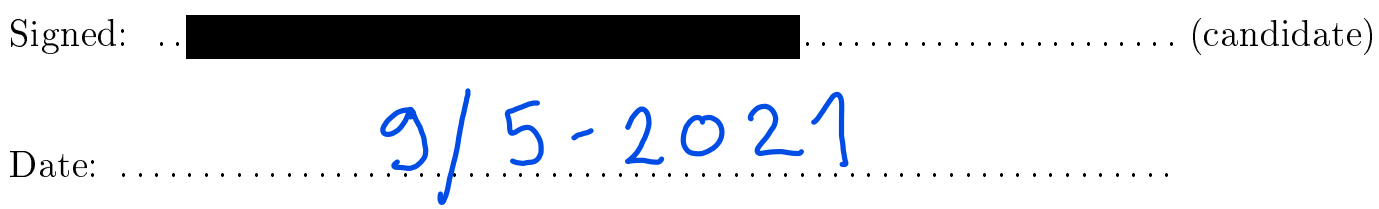

\section{Statement 2}

This thesis is the result of my own investigations, except where otherwise stated. Other sources are acknowledged by footnotes giving explicit references. A bibliography is appended.

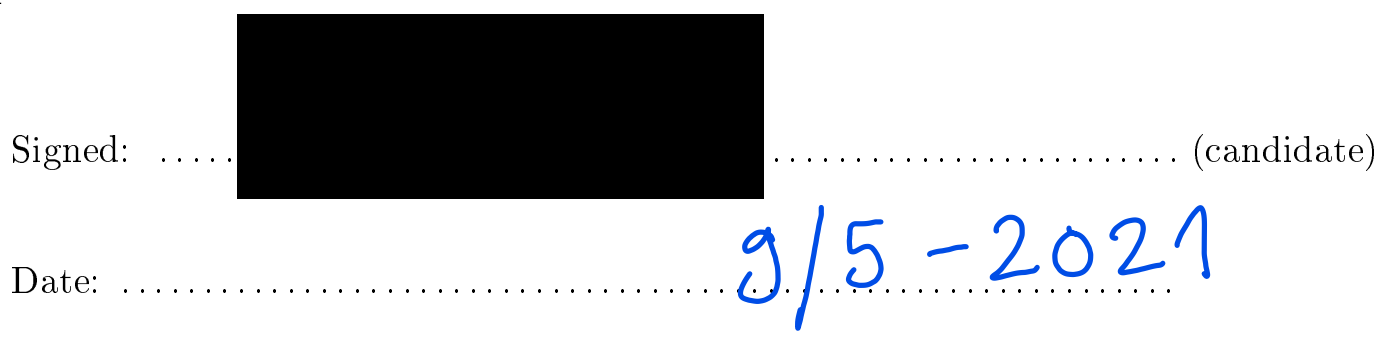

\section{Statement 3}

I give consent for the thesis, if accepted to be made available online in the University's Open Access Repository and for inter-library loan, and for the title and summary to be made available to outside organisations.

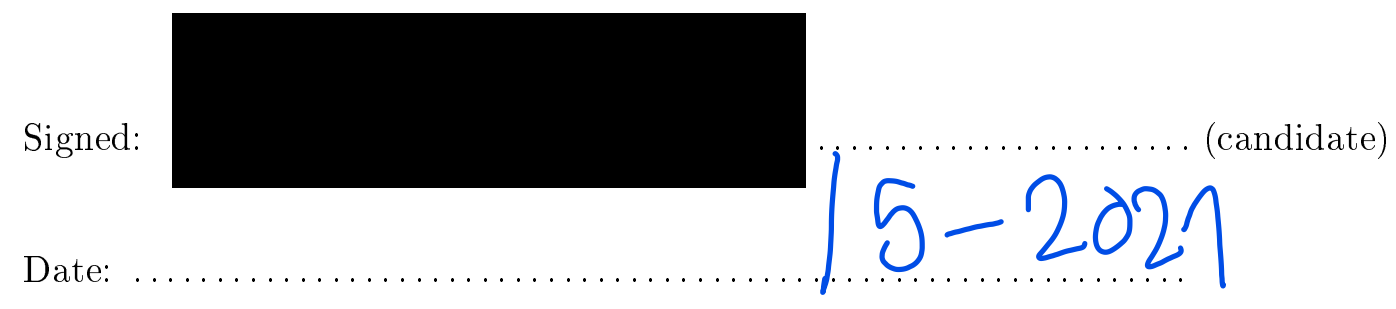




\section{Contents}

List of Abbreviations ix

List of Figures

List of Tables $\quad$ Xxvii

1 Introduction 1

1.1 Mathematical Oncology . . . . . . . . . . . . . . . . 1

1.2 Mathematics in Personalised Oncology . . . . . . . . . . . 2 2

1.3 Multiscale Models . . . . . . . . . . . . . . . . 5

2 Mathematical Framework 9

2.1 The Cellular Automaton . . . . . . . . . . . . . . . . . . 9

2.2 Cell Cycle Models . . . . . . . . . . . . . . . . . 10

2.2.1 A Mechanistic Cell Cycle Model . . . . . . . . . . . . . . 11

2.2.2 A Phenomenological Cell Cycle Clock Model . . . . . . . . . . 17

2.2.3 A Stochastic Cell Cycle Clock Model . . . . . . . . . . . . . . 18

2.3 Cell Division and Tumour Growth . . . . . . . . . . . . . . . . 19

2.4 Oxygen Dynamics . . . . . . . . . . . . . . . . 20 20

2.5 Drug Dynamics . . . . . . . . . . . . . . . . . . . 21

2.5.1 Some Basic Concepts in Pharmacology . . . . . . . . . . 21

2.5.2 Drug Binding and Drug Activation . . . . . . . . . 23

2.5.3 Cancer Drugs . . . . . . . . . . . . . . . 26

2.5.4 Drug Disposition . . . . . . . . . . . . . . 27

2.6 Radiotherapy . . . . . . . . . . . . . . . . . . . 28

2.6.1 Effects of Radiation in Biological Materials . . . . . . . . 28 
2.6.2 Influence of Oxygen on Radiotherapy Responses _ . . . . . . 2 29

2.6.3 The Linear-Quadratic Model . . . . . . . . . . . . . . . 31

3 Drug Resistance 37

3.1 Chapter Summary . . . . . . . . . . . . . . 37

3.2 Introduction . . . . . . . . . . . . . . . . 38

3.3 Model and in silico Framework . . . . . . . . . . . . . . 42

3.3 .1 Intratumoural Heterogeneity $\ldots \ldots \ldots \ldots$

$3.3 .2 \quad$ Intracellular Dynamics . . . . . . . . . . . . . . . . 45

3.3.3 Extracellular Dynamics . . . . . . . . . . . . . . . . 46

3.3.3.1 Parameters for Oxygen and Drug Distribution .... 47

3.3 .4 Drug Resistance . . . . . . . . . . . . . . . . 48

3.3.4.1 Primary Drug Resistance . . . . . . . . . . 50

3.3.4.2 Induced Drug Resistance . . . . . . . . . . 51

3.3.4.3 Communicated Drug Resistance via Exosomes . . . . 52

3.3.4.4 Cell Cycle Mediated Drug Resistance by Slow-Cycling Cells .................... 553

3.3.4.5 Parameters and Algorithms for Drug Resistance . . . . 54

3.3 .5 Implementation . . . . . . . . . . . . . . . 56

3.4 Results and Discussion . . . . . . . . . . . . . 58

3.4 .1 Results ...................... 58

3.4.1.1 No Drug Resistance . . . . . . . . . . . . . 58

3.4.1.2 Primary Drug Resistance . . . . . . . . . . . 60

3.4.1.3 Induced Drug Resistance . . . . . . . . . . 60 60

3.4.1.4 Communicated Drug Resistance via Exosomes .... . 62

3.4.1.5 Cell Cycle Mediated Drug Resistance by Slow-Cycling Cells .................... 63 63

3.4.1.6 Cell-Map Topology . . . . . . . . . . . . 63 63

3.4 .2 Discussion . . . . . . . . . . . . . . . . . . . 64

3.5 Conclusion .....................667 
4 Hypoxia-Activated Prodrugs 71

4.1 Chapter Summary . . . . . . . . . . . . . . . 71

4.2 Introduction . . . . . . . . . . . . . . . 72

4.3 Model ....................... 75

4.3.1 Mathematical Framework . . . . . . . . . . . . 76

4.3.2 Cell Cycle Progression . . . . . . . . . . . . . . 76 76

4.3 .3 Tumour Growth . . . . . . . . . . . . . . 78

4.3.4 Oxygen Distribution and Hypoxia . . . . . . . . . 78

4.3.5 Hypoxia Activated Prodrugs . . . . . . . . . . . . . . 80

4.3.5.1 Drug Parameters . . . . . . . . . . . . . 83

4.3 .6 Radiotherapy . . . . . . . . . . . . . . . . 85

4.3 .7 Parameters . . . . . . . . . . . . . . 85

4.3.8 Implementation and in silico Framework . . . . . . . . . . . 85

4.4 Results and Discussion . . . . . . . . . . . . . . . . . . . 87

4.4.1 HAP and IR Monotherapies Attack Tumours in Different Ways . 87

4.4.2 HAP-IR Treatment Scheduling Impacts HAP Efficacy in Sufficiently Hypoxic Tumours . . . . . . . . . . . . . . . . . . 89

4.4.3 HAPs Enhance Radiotherapy Effects in Sufficiently Hypoxic Tumours ....................... . . . . . .

4.4.4 The Intratumoural Oxygen Landscape Impacts HAP Efficacy . . 94

4.5 Conclusion . . . . . . . . . . . . . . . . 97

5 Bridging in vitro and in vivo research via agent-based modelling 101

5.1 Chapter Summary . . . . . . . . . . . . . . . . . . . . . 101

5.2 Introduction . . . . . . . . . . . . . . . . . . 102

5.2 .1 Bridging in vitro and in vivo research . . . . . . . . . . . . . . . 102

5.2 .2 DNA damage response inhibiting drugs . . . . . . . . . . . . 103

5.3 Model and Method . . . . . . . . . . . . . . . . . . . 104

5.3 .1 Cellular automaton lattice . . . . . . . . . . . . . . 105

5.3 .2 Cell cycle model . . . . . . . . . . . . . . . . . . . 106

5.3 .3 Cell proliferation . . . . . . . . . . . . . . . 108

5.3.4 Oxygen distribution and influence on cells . . . . . . . . . . 109

5.3.5 Drug distribution across the lattice . . . . . . . . . . . . 111 
5.3 .6 Drug responses . . . . . . . . . . . . . . . . . . 113

5.3 .7 Parameters . . . . . . . . . . . . . . . . . . 114

5.3.8 Differences between in vitro and in vivo modelling rules . . . . . 114

5.3 .9 Implementation . . . . . . . . . . . . . . . . . . . . . 115

5.4 Model Parameterisation . . . . . . . . . . . . . . . . . 115

5.4 .1 Cell doubling . . . . . . . . . . . . . . . . . 115

5.4 .2 Cell cycle progression . . . . . . . . . . . . . . 117

5.4 .3 Drug response . . . . . . . . . . . . . . . . . . . 118

5.4 .4 Cell death . . . . . . . . . . . . . . . . . . . 119

5.4 .5 In vivo calibration . . . . . . . . . . . . . . . . . . 119

5.5 Cross-Section to Tumour Spheroid Extrapolation . . . . . . . . . . . 119

5.6 Experimental Data . . . . . . . . . . . . . . . . . . . . . 120

5.7 Results . . . . . . . . . . . . . . . . . . . . 120

5.7 .1 Simulating in vitro experiments . . . . . . . . . . . . . 120

5.7 .2 Simulating in vivo experiments . . . . . . . . . . . . . 123

5.8 Discussion . . . . . . . . . . . . . . . . . . . 123

6 Uncertainty and Sensitivity Analyses

6.1 Chapter Summary . . . . . . . . . . . . . . . . . . . . 127

6.2 Introduction . . . . . . . . . . . . . . . . . 128

6.3 The Measure of Stochastic Superiority . . . . . . . . . . . . . . 130

6.3.1 The Common Language Statistic . . . . . . . . . . . . . . . . . 130

6.3.2 The A Measure of Stochastic Superiority . . . . . . . . . . . . . 130

6.4 Consistency Analysis _ . . . . . . . . . . . . . . . . 136

6.4.1 Quick Guide to Consistency Analysis . . . . . . . . . . . . 138

6.5 Robustness Analysis . . . . . . . . . . . . . . . . . 141

6.5.1 Quick Guide to Robustness Analysis . . . . . . . . . . . . . . . . 142

6.6 Latin Hypercube Sampling and Analysis . . . . . . . . . . . . . . 145

6.6.1 Latin Hypercube Sampling . . . . . . . . . . . . . . . . . 145

6.6.2 Latin Hypercube Analysis . . . . . . . . . . . . . . . . . . 148

6.6.3 Quick Guide to Latin Hypercube Sampling and Analysis . . . . 150

6.7 Case Study: Analysing the Results from Chapter 5 . . . . . . . . . . 153

6.7.1 Consistency Analysis . . . . . . . . . . . . . . . . . . . . 154 
6.7.2 Robustness Analysis . . . . . . . . . . . . . . . 155

6.7 .3 Latin Hypercube Analysis . . . . . . . . . . . . . . 156

7 Conclusion $\quad \mathbf{1 7 3}$

8 Appendix

\begin{tabular}{ll} 
References & 191 \\
\hline
\end{tabular} 


\section{List of Abbreviations}

- ABM - agent-based model

- AHAP - hypoxia activated prodrug in activated form

- APC - anaphase-promoting complex

- ATM - Ataxia telangiectasia mutated kinase

- ATR - Ataxia telangiectasia Rad3 related kinase

- BRF - bioreduction factor

- CA - cellular automaton

- $C d h 1$ - APC-Cdh1 complex

- Cdks - cyclin-dependent protein kinases

- CSC - cancer stem cell

- $C y c B$ - Cdk-cyclin B complex

- CL - common language statistics

- DNA - deoxyribonucleic acid

- DSB - double strand break

- DR - drug resistant

- ECM - extracellular matrix

- Gy - gray (1 gray = 1 Joule/kilogram $)$ 
- G0 - quiescent (non-cycling) cell state

- G1 - gap 1 part of cell cycle

- G1DF - G1-delay factor

- G2 - gap 2 part of cell cycle

- HAP - hypoxia activated prodrug

- HIF - hypoxia inducible transcription factor

- Hsps - heat schock proteins

- ICC - intercellular communication

- LQ - linear quadratic (radiotherapy response model)

- IR - ionising radiation

- M - mitosis part or cell cycle phase

- MDR - multi-drug resistance

- MCTS - multi-cellular tumour spheroid

- ODE - ordinary differential equation

- OER - oxygen enhancement ratio

- OMF - oxygen modification factor

- PDE - partial differential equation

- Plk1 - Plk1 protein in active form

- $\mathrm{pO}_{2}$ - partial oxygen pressure

- PI3K - phosphoinositide 3-kinase

- $p 55 c d c_{A}$ p 55 cdc-APC complex in active form

- $p 55 c d c_{T}$ p $55 c d c-A P C$ complex in total form 
- S - synthesis part or cell cycle phase (in Chapters 2,4,5)

- S - drug sensitive (in Chapter 3)

- SC - slow cycling (cell)

- Syn - synthesis part or cell cycle phase (in Chapter 3)

- SSB - single strand break 


\section{List of Figures}

1.1 Personalised cancer practice on various levels, namely Population, Patient, Tumour Profile. On a population macro-level, a population can be categorised into various subpopulations (for instance based on age, gender or smoking habits), which in turn can be evaluated and risk-assessed for preventative, detective and corrective oncology practice. On a central, patient-specific level, cancer care may be tailored to fit the needs, lifestyle and priorities of the patient, in pursuit of medicine that optimises both treatment outcome and the patient's quality of life. On a tumour profile level, tumour-specific data can provide information which may contribute towards disease prognoses and intelligent treatment decisions. Narrowing down cancer care personalisation to tumour level allows for a bottom-up approach to personalised tumour treatments. . . . . . . . .

1.2 'Bench-to-bedside' depicts the practice of transferring in vitro and in vivo findings from the laboratory to a clinical setting. The contemporary mathematician works on both blackboards and computer keyboards thus we therefore, analogously, present the term blackboard-to-bedside to describe the action of translating mathematical and computational intelligence to clinical application.

2.1 Time evolution of protein concentrations in one simulated cancer cell (measured in grams of protein per gram of total cell mass), where the cell cycle length is 25 hours. (a) The opposing dynamics between $[C y c B]$ and $[C d h 1]$, which is key in the used mathematical cell cycle model. (b) Concentrations of the auxiliary proteins $[P k l 1]$ and $\mathrm{p} 55 \mathrm{cdc}$ in its total form $\left[p 55 c d c_{T}\right]$, and its active form $\left[p 55 c d c_{A}\right]$. (c) Cell mass, [mass] over time, where [mass] is halved at the very start of each cell cycle, i.e. immediately after cell division. . . . . . . . . . 


\section{LIST OF FIGURES}

2.2 The G1 Delay Factor (G1DF) is incorporated in the model to achieve oxygendependent G1 arrest. The G1DF (dark line) is approximated using results (red crosses) from a previous mathematical study by Alarcon et al. [1]. . . . . . .

2.3 The 1st, 2nd and 3rd order neighbourhood (O.N.) of a parental agent using (a) the von Neumann convention and (b) the Moore convention. . 20

2.4 The target occupancy $\left(p_{A}\right)$ plotted over drug concentration $\left(x_{A}\right)$ when the drug concentration increases on a linear (left) and logarithmic (right) scale. Here $K_{A}$, i.e. the drug concentration that yields $50 \%$ target occupancy, is $x_{A}=100$

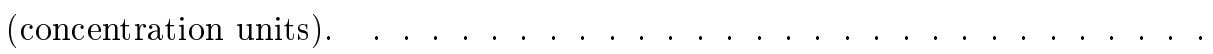

2.5 The oxygen enhancement ratio (OER) and the oxygen modification factor (OMF) plotted over oxygenation value. . . . . . . . . . . .

2.6 The probability $S(x, t)$ that a cell location $x$ at time $t$ in our mathematical framework survives a radiation dose of $2 \mathrm{~Gy}$, as a function of its cell cycle phase (see legend) and oxygenation value. The values for $\alpha$ and $\beta$ are gathered from a previous study by Kempf et al. [2] and are listed in Table 4.1 . . . . . 36

3.1 A schematic representation of the multiscale hybrid mathematical model used in this study. The model integrates extracellular, intracellular and intercellular dynamics. This is an on-lattice model and a lattice point may be occupied by a sensitive (S) or drug resistant (DR) cancer cell shown in black, a blood vessel cross-section or extracellular space. Various categories of drug resistance regarded on a cellular resolution are incorporated in the model, these categories are marked in red and listed in Table $3.4 \ldots \ldots \ldots$

3.2 Classification of drug resistance categories occurring in the model. Drug resistance may be independent of drug presence (orange labels), induced as a consequence of drug presence (purple labels), or either (orange and purple labels). Cell acquisition of any DR phenotypical trait (top half) is here modelled as irreversible and inheritable by any future daughter cells to the cell in question. . . . . . . . . . . . . . 50

3.3 Partial algorithm for determining primary drug resistance. . . . . 55

3.4 Partial algorithm for determining induced drug resistance. . . . . . 5 56

3.5 Partial algorithm for determining exosome production. . . . . . . 5 56 
3.6 Partial algorithm for determining the spontaneous conversion from a slow-cycling to a fast-cycling state. . . . . . . . . .

3.7 Growth of the cancer cell population over time in drug absence, showing total population (black graph), drug sensitive subpopulation (blue graph) and drug resistant subpopulation (red graph) incorporating control-case drug resistance categories (a0) No DR, (b0) Primary DR and (e0) SlowCycling DR. Cases (c0) Drug-induced DR and (d0) Communicated (ICC) DR are omitted since they produce the same results as (a0) in drug absence. Each graph shows the average number of cells based on 100 simulations, the standard deviation is demonstrated with error bars. . .

3.8 Growth of the cancer cell population over time when drugs are applied at 500 and 600 hours, showing total population (black graph), drug sensitive subpopulation (blue graph) and drug resistant subpopulation (red graph). Each row in the figure corresponds to a category of drug resistance (a) to (e) according to the labelling in Table 3.4 ((a) No DR, (b) Primary DR, (c0) Drug-induced DR, (d0) Communicated DR (e) Slowcycling DR), whilst each column corresponds do a specific drug dosage varying from low (leftmost column) to high (rightmost column), namely (1) 1C, (2) 2C, (3) 4C and (4) 8C according to the labelling system in Table 3.5. Each graph shows the average number of cells based on 100 simulations, the standard deviation is demonstrated with error bars. . .

3.9 The scaling efficiency, demonstrating the relationship between drug dosage and drug efficacy in terms of number of killed cancer cells. This is for the first drug administration at 500 hours for experiments (a1) to (a4) according to the labelling in Tables 3.4 and 3.5 where no drug resistant phenotypes are present. Results are based on average values for 100 tests. . . . . . . . . . . .

3.10 Cell-maps of the cancer cell populations at times $500 \mathrm{~h}$ (immediately before first drug dose), $510 \mathrm{~h} 600 \mathrm{~h}$ (immediately before second drug dose), $610 \mathrm{~h}$ and $700 \mathrm{~h}$ (end of simulation). Cases incorporating (a) No DR and (b) Primary DR are shown for drug dosages of (1) $1 \mathrm{C}$ and (4) 8C according to the labelling in Tables 3.4 and 3.5. White areas correspond to extracellular space. . . . . . . . . . . . . . . . 


\section{LIST OF FIGURES}

3.11 Cell-maps of the cancer cell populations at times $500 \mathrm{~h}$ (immediately before first drug dose), $510 \mathrm{~h} 600 \mathrm{~h}$ (immediately before second drug dose), $610 \mathrm{~h}$ and $700 \mathrm{~h}$ (end of simulation). Cases incorporating (c) Induced DR, (d) ICC DR and (e) SC DR are shown for drug dosages of (1) $1 \mathrm{C}$ and (4) $8 \mathrm{C}$ according to the labelling in Tables 3.4 and 3.5. White areas correspond to extracellular matrix. . . . . . . . . . . .

3.12 Diagram of the drug sensitive subpopulation (blue) and the drug resistant subpopulation. Various types of drug resistance are incorporated namely (a) No DR, (b) Primary DR, (c) Induced DR, (d) ICC DR, (e) SC DR. Results are shown at times $500 \mathrm{~h}$ (immediately before the first drug dose), $510 \mathrm{~h}, 600 \mathrm{~h}$ (immediately before the second drug dose), $610 \mathrm{~h}$ and $700 \mathrm{~h}$ (end of simulation) for low drug dosages, (1) 1C, and high drug dosages, (4) $8 \mathrm{C}$ according to the labelling in Table 3.5. Each diagram shows the

3.13 Diagram of the ratio between the drug sensitive subpopulation (blue) and the drug resistant sub-population. Various types of drug resistance are incorporated namely (a) No DR, (b) Primary DR, (c) Induced DR, (d) ICC DR, (e) SC DR. Results are shown at times $500 \mathrm{~h}$ (immediately before the first drug dose), $510 \mathrm{~h}, 600 \mathrm{~h}$ (immediately before the second drug dose), $610 \mathrm{~h}$ and $700 \mathrm{~h}$ (end of simulation) for low drug dosages, (1) 1C, and high drug dosage, (4) 8C according to the labelling in Table 3.5 Each diagram shows the average value based on 100 simulations. . . . .

3.14 Tumours exposed to chemotherapeutic drugs given in low (top) or high (bottom) dosages, the latter dosage option kills more cancer cells. One of the tumours (left) expresses drug resistance pre chemotherapy, the other one (right) does not. Generally in our in silico experiment, drug resistance that occurs independently of the drug, and thus may precede chemotherapy, is amplified by high drug dosages (left). Conversely, drug resistance that is induced by drug presence is accelerated by low to moderate drug dosages (right). . . . . . . . . . . . . . . . . 69 69 
4.1 Cell count over time for tumour spheroids. The in silico data is based on 10 simulations runs, the mean (black line) shows the average value and the gray ribbon shows standard deviation. In vitro data (red error bars) are extracted from plots produced by Voissiere et al. [3] using a Java

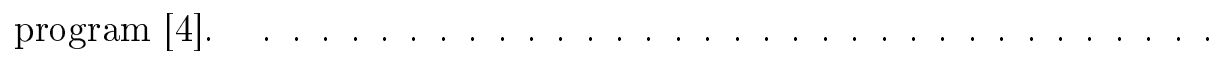

4.2 Top: Images from in vitro experiments performed by Voissiere et al. [3], in which cell nuclei are stained blue and furthermore proliferative cells are stained green by the proliferation marker Ki-67. Bottom: Images from in silico experiments performed in this study, where proliferative (cycling) cells are coloured green and quiescent cells are coloured blue. .

4.3 Top: Images from in vitro experiments performed by Voissiere et al. [3], in which hypoxic cells are stained green by pimonidazole and normoxic cells are stained blue. Bottom: Images from in silico experiments performed in this study, where hypoxic cells $\left(p_{\mathrm{O}_{2}} \leq 10 \mathrm{mmHg}\right)$ are coloured green and normoxic cells $\left(p_{\mathrm{O}_{2}}>10 \mathrm{mmHg}\right)$ are coloured blue. . . . . .

4.4 The bioreduction factor, $B R F$, expresses the fraction of HAP compound that reduces to AHAP compound within one hour as a function of oxygenation (measured in $\mathrm{mmHg}) \ldots \ldots \ldots \ldots$

4.5 The 'Small' (20 day old) MCTS and the 'Large' (30 day old) MCTS are used in Sections 4.4.1, 4.4.2, 4.4.3 to allow for comparisons in treatment responses between tumours with different oxygenation levels. Top: Simulation snapshots of the MCTSs at the time point $T_{0}$ when treatments commence (A1: Small MCTS, B1: Large MCTS). Hypoxic cells $\left(p_{\mathrm{O}_{2}} \leq 10 \mathrm{mmHg}\right)$ are green whilst normoxic cells are blue. Middle: Oxygen histograms at time $T_{0}$, in which hypoxic cell counts are shown in green and normoxic cell counts are shown in blue (A2: Small MCTS, B2: Large MCTS). Bottom: Cell-cycle phase histograms at time $T_{0}$ (A3: Small MCTS, B3: Large MCTS). . . . . . . . . . . . .

4.6 Treatment responses for HAPs (left) and IR (right) monotherapies for the 'Small' (top) and 'Large' (bottom) MCTS. The monotherapy is given at 0 hours. Graphs demonstrate cell-cycle specific cell count (i.e. number of viable, undamaged cells) over time. Solid lines show mean values, and ' + ' markers show standard deviations for 10 in silico runs. . . . . . . . . . 


\section{LIST OF FIGURES}

4.7 Treatment responses for HAPs (left) and IR (right) monotherapies for the 'Small' (top) and 'Large' (bottom) MCTS. The monotherapy is given at 0 hours. Graphs demonstrate cell-cycle specific composition (of viable, undamaged cells) over time. Solid lines show mean values for 10 in silico runs (standard deviations are negligible hence not shown) . . . . . . .

4.8 Treatment responses for HAPs (left) and IR (right) monotherapies for the 'Small' (top) and 'Large' (bottom) MCTS. Histograms over cellular oxygenation levels at time $T_{0}$ (monotherappy administration time) and 4 hours later are shown. Results are based on mean values from $10 \mathrm{in}$

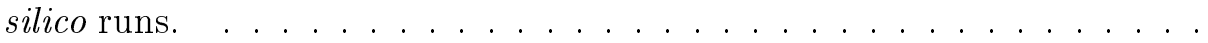

4.9 Treatment responses (in terms of cell count) for HAP-IR combination therapies in the 'Small' MCTS (left) and the 'Large' MCTS (right). One dose of HAPs and one dose of IR are administered at various schedules. Solid and dashed lines show mean values, and ' + ' markers show standard deviations for 10 in silico runs. . . . . . . . . . . . . . . . . . . .

4.10 Treatment responses of radiotherapy in various MCTSs when either (1) an IR monotherapy dose is administered at $T_{0}+48$ hours or (2) IR is given at $T_{0}+48$ hours following a prior HAP dose at time $T_{0}$. Note that only explicit IR responses (not HAP responses) are shown. The oxygen-levels of the 'Large' (left) and 'Small' (right) tumours are scaled by a factor of 1 (least hypoxic), 1/2 or $1 / 4$ (most hypoxic). The value calibrated from in vitro experiments [3] correspond to the scaling with factor 1. Yellow bars show number of viable cells (instantaneously) before IR administration, blue bars show the number of viable cells (instantaneously) post IR. Red bars show how many cells (as a fraction) survived the IR attack. . . . .

4.11 Top: MCTS A and B prior to treatment commencing. The MCTSs are visualised in both opaque and transparent formats. Bottom: Oxygen histograms for MCTS A and B prior to treatment commencing, hypoxic $\left(p_{\mathrm{O}_{2}} \leq 10 \mathrm{mmHg}\right)$ cell counts are shown in green and normoxic cell counts are shown in blue. . . . . . . . . . . . . . . 
4.12 Treatment responses in MCTS A and MCTS B when HAPs are administered at 0 hours. The number of viable (undamaged) cells are plotted over time for MCTS A and MCTS B tumour. Activator cells $\left(p_{\mathrm{O}_{2}}=0\right.$ $\mathrm{mmHg})$ are shown in dashed lines and bystander $\left(p_{\mathrm{O}_{2}}=100 \mathrm{mmHg}\right)$ cells shown in solid lines. Results show mean values for 10 in silico runs and '十' markers show standard deviations. . . . . . . . . . . . . . .

5.1 A schematic of the mathematical modelling approach used in this study. An agent-based mathematical model, that distinguishes between in vitro and in vivo modelling rules, is used to bridge the gap between in vitro and in vivo research. In this study, the mathematical model is first parameterised by in vitro data and is thereafter used to predict in vivo outcomes. . . . . . . . . . . . . . . . . . . . 103

5.2 Cell cycle model: An agent, i.e a cell (in vitro) or a group of cells (in vivo), progresses through various states of the cell cycle, where the states correspond to cell cycle phases and are shown as nodes in the graph. Viable (undamaged or damaged) states are shown in circles, whilst the dead state is shown as a cross. Paths illustrate transitions between states, and symbols next to the paths denote the probabilities that the paths will be taken. The dashed path can be inhibited by an ATR inhibiting drug, such as AZD6738. . . . . . . . . . . . . . . 107

5.3 A summary of the differences between the in vitro and in vivo rules used in the mathematical framework. . . . . . . . . . . . . . . . 116

5.4 Simulated in vitro drug response curves. LoVo cells are exposed to drug (AZD6738) at 0 hours. Left: The percentage of $\gamma \mathrm{H} 2 \mathrm{AX}$-positive (DNAdamaged) cells in the system over time. Right: Cell count over time. Simulated mean values and standard deviations for 100 in silico runs are shown with solid lines and shaded ribbons respectively. In vitro mean values and standard deviations are demonstrated with center points and

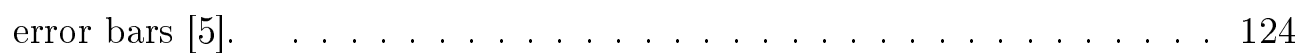




\section{LIST OF FIGURES}

5.5 Simulated in vivo drug response curves. LoVo xenografts are exposed to drug (AZD6738) once daily for 14 days. Left: The percentage of $\gamma \mathrm{H} 2 \mathrm{AX}$ positive (DNA-damaged) cells in the xenograft over time. Right: Tumour volume over time. Simulated mean values and standard deviations for 100 in silico runs are shown with solid lines and shaded ribbons respectively. In vivo mean values and standard errors are demonstrated with center points and error bars $[5] . \ldots \ldots \ldots$. . . . . . . . . . . . . . . . .

6.1 Using Equation 6.8 to compute the point estimate of the A-measure, i.e the Â-measure or $\hat{A}_{B, C}$, of the two distributions of data samples $B$ and $C$ with sizes $m$ and $n$ respectively. . . . . . . . . . . . . 134

6.2 The small (left), medium (centre) and large (right) threshold values for the scaled A measure of stochastic superiority $\left(\underline{\hat{A}}_{B C}\right)$ are based on Cohen's $\mathbf{d}$-values comparing two normal distributions $B$ and $C$ with the same variance. The higher the overlap between $B$ and $C$, the smaller the d-value, and the smaller the $\underline{\hat{A}}_{B C}$-measure $\left(\underline{\hat{A}}_{B C} \in[0.5,1]\right) . \ldots \ldots 136$

6.3 3-step quick guide on how to perform Consistency Analysis. . . . . . . . 141

6.43 -step quick guide on how to perform Robustness Analysis. . . . . . . . 144

6.5 Left: An $\ell \times \ell$ Latin Square in which each Latin symbol occurs $\ell$ times, exactly once in each row and exactly once in each column. Right (analogously): A two-dimensional parameter space spanned by the input parameters $p^{1}$ and $p^{2}$ that are both sectioned into $\ell$ intervals. Using Latin Hypercube sampling, $\ell$ parameter combinations $\left(p_{j}^{1}, p_{j}^{2}\right)$ are sampled where $j=1,2, . ., \ell$ and each $p^{1}$-interval is sampled from exactly once and each $p^{2}$-interval is sampled from exactly once. . . . . . . . . . . 146

6.6 3-step quick guide on how to perform Latin Hypercube Sampling and Analysis. . . . . . . . . . . . . . . . . . . . 153

6.7 Consistency Analysis. $\hat{A}$-values in initial (top) and scaled (bottom) form for distribution size $n=1 . \ldots \ldots \ldots \ldots$. . . . . . . . . . . . . . .

6.8 Consistency Analysis. $\hat{A}$-values in initial (top) and scaled (bottom) form

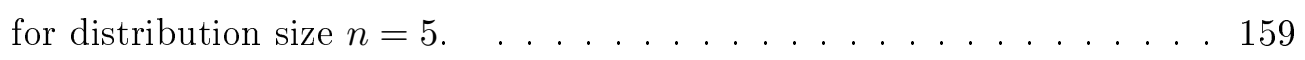

6.9 Consistency Analysis. $\hat{A}$-values in initial (top) and scaled (bottom) form for distribution size $n=50 \ldots \ldots \ldots \ldots \ldots \ldots$ 
6.10 Consistency Analysis. $\hat{A}$-values in initial (top) and scaled (bottom) form

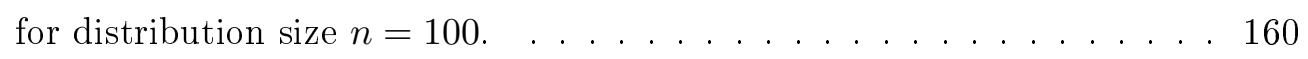

6.11 Consistency Analysis. $\hat{A}$-values in initial (top) and scaled (bottom) form

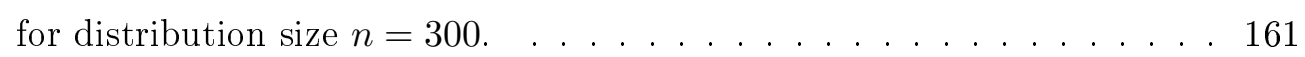

6.12 Consistency Analysis. Scaled $\hat{A}$-values for various distribution sizes tested in the Consistency Analysis. . . . . . . . . . . . . . . 161

6.13 Robustness Analysis. Left: Output responses, in terms of percentage of $\gamma \mathrm{H} 2 \mathrm{AX}$ positive (i.e. damaged) cells, and cell count as a result of perturbations to the input variable $\mu$. Right: Maximal $\hat{A}$-values resulting from comparisons between distributions with perturbed data and a distribution with calibrated (unperturbed) data. . . . . . . . . . . . 162

6.14 Robustness Analysis. Left: Output responses, in terms of percentage of $\gamma \mathrm{H} 2 \mathrm{AX}$ positive (i.e. damaged) cells, and cell count as a result of perturbations to the input variable $\sigma$. Right: Maximal $\hat{A}$-values resulting from comparisons between distributions with perturbed data and a distribution with calibrated (unperturbed) data. . . . . . . . . . . 163

6.15 Robustness Analysis. Left: Output responses, in terms of percentage of $\gamma \mathrm{H} 2 \mathrm{AX}$ positive (i.e. damaged) cells, and cell count as a result of perturbations to the input variable $\Pi_{D-s}$. Right: Maximal $\hat{A}$-values resulting from comparisons between distributions with perturbed data and a distribution with calibrated (unperturbed) data. . . . . . . 164

6.16 Robustness Analysis. Left: Output responses, in terms of percentage of $\gamma \mathrm{H} 2 \mathrm{AX}$ positive (i.e. damaged) cells, and cell count as a result of perturbations to the input variable $\Theta_{D-S}$. Right: Maximal $\hat{A}$-values resulting from comparisons between distributions with perturbed data and a distribution with calibrated (unperturbed) data. . . . . . . 165

6.17 Robustness Analysis. Left: Output responses, in terms of percentage of $\gamma \mathrm{H} 2 \mathrm{AX}$ positive (i.e. damaged) cells, and cell count as a result of perturbations to the input variable $E C_{50}$. Right: Maximal $\hat{A}$-values resulting from comparisons between distributions with perturbed data and a distribution with calibrated (unperturbed) data. . . . . . . . 166 


\section{LIST OF FIGURES}

6.18 Robustness Analysis. Left: Output responses, in terms of percentage of $\gamma \mathrm{H} 2 \mathrm{AX}$ positive (i.e. damaged) cells, and cell count as a result of perturbations to the input variable $\gamma$. Right: Maximal $\hat{A}$-values resulting from comparisons between distributions with perturbed data and a distribution with calibrated (unperturbed) data. . . . . . . . . 167

6.19 Robustness Analysis. Left: Output responses, in terms of percentage of $\gamma \mathrm{H} 2 \mathrm{AX}$ positive (i.e. damaged) cells, and cell count as a result of perturbations to the input variable $T_{D \rightarrow L}$. Right: Maximal $\hat{A}$-values resulting from comparisons between distributions with perturbed data and a distribution with calibrated (unperturbed) data. . . . . . . 168

6.20 Latin Hypercube Analysis. Outputs in terms of $\gamma \mathrm{H} 2 \mathrm{AX}$ positive cells (left) and number of viable cells (right) when global parameter perturbations are performed. The scatter-plots show the correlation between outputs and the input variable $\mu \ldots \ldots \ldots \ldots$

6.21 Latin Hypercube Analysis. Outputs in terms of $\gamma \mathrm{H} 2 \mathrm{AX}$ positive cells (left) and number of viable cells (right) when global parameter perturbations are performed. The scatter-plots show the correlation between outputs and the input variable $\sigma . \ldots \ldots \ldots \ldots \ldots$

6.22 Latin Hypercube Analysis. Outputs in terms of $\gamma \mathrm{H} 2 \mathrm{AX}$ positive cells (left) and number of viable cells (right) when global parameter perturbations are performed. The scatter-plots show the correlation between outputs and the input variable $\Pi_{D-S} . \ldots \ldots \ldots \ldots \ldots$

6.23 Latin Hypercube Analysis. Outputs in terms of $\gamma \mathrm{H} 2 \mathrm{AX}$ positive cells (left) and number of viable cells (right) when global parameter perturbations are performed. The scatter-plots show the correlation between outputs and the input variable $\Theta_{D-S} . \ldots \ldots \ldots \ldots \ldots$

6.24 Latin Hypercube Analysis. Outputs in terms of $\gamma \mathrm{H} 2 \mathrm{AX}$ positive cells (left) and number of viable cells (right) when global parameter perturbations are performed. The scatter-plots show the correlation between outputs and the input variable $E C_{50} \ldots \ldots \ldots \ldots \ldots$ 
6.25 Latin Hypercube Analysis. Outputs in terms of $\gamma \mathrm{H} 2 \mathrm{AX}$ positive cells (left) and number of viable cells (right) when global parameter perturbations are performed. The scatter-plots show the correlation between outputs and the input variable $\gamma . \ldots \ldots \ldots \ldots$. . . . . . . . . . .

6.26 Latin Hypercube Analysis. Outputs in terms of $\gamma \mathrm{H} 2 \mathrm{AX}$ positive cells (left) and number of viable cells (right) when global parameter perturbations are performed. The scatter-plots show the correlation between outputs and the input variable $T_{L \rightarrow D} \ldots \ldots \ldots \ldots \ldots \ldots$

8.1 Cell-maps (top) and oxygen-maps (bottom) at certain times pre chemotherapy administration. The oxygen-maps demonstrate the spatial oxygen distribution in terms of scaled, nondimensionalised oxygen values ranging between 0 and 1 at each grid point. . . . . . . . . . . . 178

8.2 Sensitivity analysis, showing the number of sensitive (blue) and drug resistant (red) cells at three time points when low (left) and high (right) drug dosages are administered, namely $1 \mathrm{C}$ and $8 \mathrm{C}$ respectively. Each test is performed 100 times and the parameters used in each test are listed in Table 8.2 Thus for Primary DR, only the parameter $\alpha_{p r i}$ is varied. For Induced DR, $\chi_{\text {ind }}$ and $\tau$ are both varied, one at a time, according to Table 8.2. For ICC DR only $a_{e x}$ is varied and similarly for SC DR only $a_{S C}$ is varied.

8.3 A flowchart of the code used in Chapter 3. . . . . . . . . . . . . 183

8.4 Scheduling of HAP-IR combination treatments, Complement to Figure 4.9. Cells are removed from the lattice instantaneously after the lethal event occurred. . . . . . . . . . . . . . . . . . . . . 184

8.5 Scheduling of HAP-IR combination treatments, Complement to Figure 4.9. Cells are removed from the lattice after a time corresponding to their doubling time $\left(\tau_{i}\right)$ post the lethal event occurred. . . . . . . . . . . 185

8.6 Treatment responses of radiotherapy in various MCTSs when either (1) an IR monotherapy dose is administered at $T_{0}+48$ hours or (2) IR is given at $T_{0}+48$ hours following a prior HAP dose at time $T_{0}$. Complement to Figure 4.9. Cells are removed from the lattice instantaneously after the lethal event occurred. . . . . . . . . . . . . . . 186 


\section{LIST OF FIGURES}

8.7 Treatment responses of radiotherapy in various MCTSs when either (1) an IR monotherapy dose is administered at $T_{0}+48$ hours or (2) IR is given at $T_{0}+48$ hours following a prior HAP dose at time $T_{0}$. Complement to Figure 4.10. Cells are removed from the lattice after a time corresponding to their doubling time $\left(\tau_{i}\right)$ post the lethal event occurred. . . . . . . . 187

8.8 A flowchart of the code used in Chapter 4. . . . . . . . . . . 188

8.9 A flowchart of the code used in Chapter 5 simulating in vitro scenarios. 189

8.10 A flowchart of the code used in Chapter 5 simulating in vivo scenarios. 190 


\section{List of Tables}

2.1 Parameters for the nondimensionalised form of Equation 1. As described in Section $2.4 \hat{K}$ denotes oxygenation value. . . . . . . . . . . 12

3.1 A table of mathematical models (referenced in Chapter 3.2) that include various aspects of cancer growth. A check mark $(\checkmark)$ indicates that the referenced model uses/includes/is what is specified in the respective

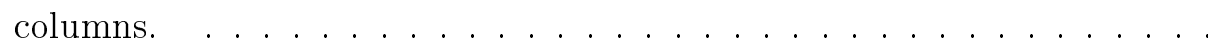

3.2 Parameters for Equations 3.1 and 3.2 (1) $\phi_{k}$ is computed using the relationship $L=\sqrt{D / \phi}$ [6]. (2) $D_{c}$ is computed using the relationship $D_{c} / D_{k}=\sqrt{\text { molecular weight of } \mathrm{O}_{2}} / \sqrt{\text { molecular weight of drug }}$ [7], where molecular weight are gathered from the PubChem data base [8].

3.3 Parameters concerning drug resistance used in the in silico framework. $\alpha_{p r i}$ denotes the probability that a cell that is placed on the lattice is drug resistant (when simulating scenarios involving primary drug resistance). $\chi_{\text {ind }}$ and $\tau$ respectively denote the drug concentration and time required to induce drug resistance in a cell (when simulation drug induced drug resistance). $\alpha_{e x}$ denotes the probability that a drug resistant cell will produce an exosome that induces drug resistance in a nearby cell (when simulating communicated drug resistance). $\alpha_{S C}$ denotes the probability that a cell will convert to a slow-cycling state (when simulating slowcycling mediated drug resistance) . . . . . . . . . . . . . 5 54

3.4 The labelling of drug resistance categories occurring in the model. . . . 5 58

3.5 The labelling of drug dosages used in the implementation. For non-zero drug dosages the labelling system is such that label $\ell$ corresponds to drug dosage $2^{\ell-1} \ldots \ldots \ldots \ldots \ldots \ldots \ldots \ldots$ 
4.1 A summary of model parameters used in the mathematical framework. . 86

5.1 In vitro calibrated parameters. . . . . . . . . . . . . . . . . . . 114

5.2 In vitro data gathered from a previous study by Checkley et al. [5]. . . . 121

5.3 In vivo data for DNA damage gathered and adapted from a previous study published by Checkley et al. [5]. . . . . . . . . . . . . 122

5.4 In vivo data for tumour volume gathered and adapted from a previous study published by Checkley et al. 5 . . . . . . . . . . . . 122

6.1 Suggested descriptor threshold values for the magnitude of the correlation coefficient, $|r|$, reported in the literature. . . . . . . . . . . 150

6.2 Maximal scaled $\hat{A}$-values produced in the Consistency Analysis for various distribution sixes $n$. The output variables are $X_{1}$, corresponding to the percentage of $\gamma \mathrm{H} 2 \mathrm{AX}$ positive (i.e. damaged) cells, and $X_{2}$, corresponding to the cell count. . . . . . . . . . . . . . 154

6.3 Pearson Product Moment Correlation Coefficients between input and output variables obtained in the Latin Hypercube Analysis. . . . . . . . 158

8.1 Nondimensionalised oxygen values of the system at certain time points, in both absolute form and scaled form (hat notation). . . . . . . . . 179

8.2 Parameters used in testing the robustness of the model. . . . . . . . . 181 


\section{Chapter 1}

\section{Introduction}

Mathematical oncology is the exciting research field in which mathematics meets traditional cancer research. This chapter motivates the use and development of mathematical tumour models, and highlights their current and potential contributions

to oncology.

\subsection{Mathematical Oncology}

Mathematical and computational approaches may constitute a fantastic complement to classical cancer research, traditionally performed in wet labs and clinics. Due to recent advances in imaging techniques, the vast accumulation of experimental and clinical data and available computational power, in silico studies have gradually been entering the stage of medical research over the last decades [9]. Cancer is a highly complex disease, and whilst this complexity presents difficulties in model formulation, parametrisation and implementation for the mathematician, this complexity also infers that there is possible biomedical insight to be gained from mathematical models and their corresponding in silico experiments. Modelling may unveil new, important information regarding biological cancer systems and their sub-mechanisms and thus elucidate underlying tumour processes [10]. The advantages of mathematical and computational oncology are multifold. Compared to other types of experiments, in silico experiments are both cheap and quick to perform, highly adaptable and associated with few direct ethical concerns [11. Theories formulated in laboratories or clinics can be tested in silico on simulated, virtual tumours prior to, or in parallel with, classical bench experiments in order to 


\section{INTRODUCTION}

validate or guide in vitro and in vivo experiments.

Today, there exists a wide array of mathematical models that are able to capture various phases of tumour progression and associated mechanisms such as tumour growth, invasion and metastasis [12-17], angiogenesis [18-21] and treatment responses [22,-28]. A comprehensive overview of the field may be found in a review article by Lowengrub et al. [29]. Some of these models have successfully conferred with both in vitro and in vivo experiments or clinical observations [5, 30-32, and consequently mathematical tumour modelling is steadily gaining acceptance in the medical community.

\subsection{Mathematics in Personalised Oncology}

Personalised medicine is becoming an increasing part of modern cancer care [33, 34]. Patient specific metrics advise contemporary clinical procedure in terms of vaccine recommendations, screening practice [35] and treatment planning [36]. The aim of personalised medicine is to tailor health care specifically to the individual patient, in pursuit of optimal treatment outcome and quality of life. As a strategy, personalised medicine can be highly beneficial in cancer care, as cancer is a disease presenting with high variability across incidences. It is indeed well established that a "one to fit all" strategy to prevent, diagnose and treat cancer is a sub-par approach [37]. Ideally, in line with concepts of personalised medicine, patients should instead be individually evaluated and matched with appropriate cancer care strategies. The personalisation of medicine can occur on various levels, as is pictorially illustrated in Figure 1.1. Patient and tumour metrics gathered from macro-level population data, down to micro-level molecular tumour data, may aid anti-cancer decision making in clinical settings.

On a population level, a population can be categorised and divided into various subpopulations, which in turn can be evaluated and risk-assessed. Certain subpopulations express elevated risks of developing particular cancer types, and likewise certain subpopulations have a predisposition to aggressive disease. This categorisation can be determined by inflexible parameters such as age [35, 36, 38, 39], genetics [38, 40], race [38] and gender [35, 39] as well as by flexible parameters such as smoking habits [41], 


\subsection{Mathematics in Personalised Oncology}

hormone exposure [40], obesity levels [42] and socio-economic factors. The combination of wide population data and gathered clinical experience can be used to determine suitable, personalised treatment strategies post tumour detection, and also to evaluate the need for cancer screening and vaccine administration [43]. For the individual patient to benefit from screenings, screening scheduling should be personalised in order to enable optimised cancer intervention [41]. By deploying mathematical models, which incorporate biological knowledge and evolutionary concepts, optimised and personalised screening recommendations can be achieved [41. In order to more competently consider the concerns, quality of life and well-being of a patient, cancer care can be tailored on a patient-specific level. Post cancer detection, lifestyle, personal priorities and economic factors all contribute towards determining which treatment strategy is the most appropriate for the individual patient [38]. On this central, patient-specific level of treatment personalisation, the dialogue between patient and clinician is of the essence [38], and it is important that the patient is well-informed by the clinician.

In parallel, in order to keep the clinician as well-informed as possible, personalised medicine can be even further detailed and narrowed down to the tumour level [44]. Post tumour detection, disease forecasting and treatment decisions can be informed by tumour-specific data. Due to the high variability of cancer displayed across disease incidences, previous research indicates that tumour prognosis and treatment responses may correlate higher with molecular tumour specifics than with larger-scale factors, such as anatomical tumour origin [33] or metrics quantified on a patient or population level. Recent advances in bio-marker handling [45, 46], biopsy techniques and medical imaging enable tumour assessment [47] prior to and throughout treatments regimes. However, current biopsy procedures may in certain cases be infeasible to perform and furthermore, tumours are highly evolutive systems whose profiles may change after an evaluation of imaging or biopsy results. Therefore, being able to predict tumour evolution, progression and treatment response, given tumour-specific input data at an earlier time point, would present an immensely valuable tool in clinical treatment planning. Various aspects of tumour growth and treatment responses are currently being investigated in silico by the mathematical oncology community [48]. One of the main, current missions in the research field of mathematical and computational oncology is to bridge 


\section{INTRODUCTION}

the gap between virtual and physical tumour control, in order for preclinical and clinical applications to directly benefit from recent advances made in the research field.

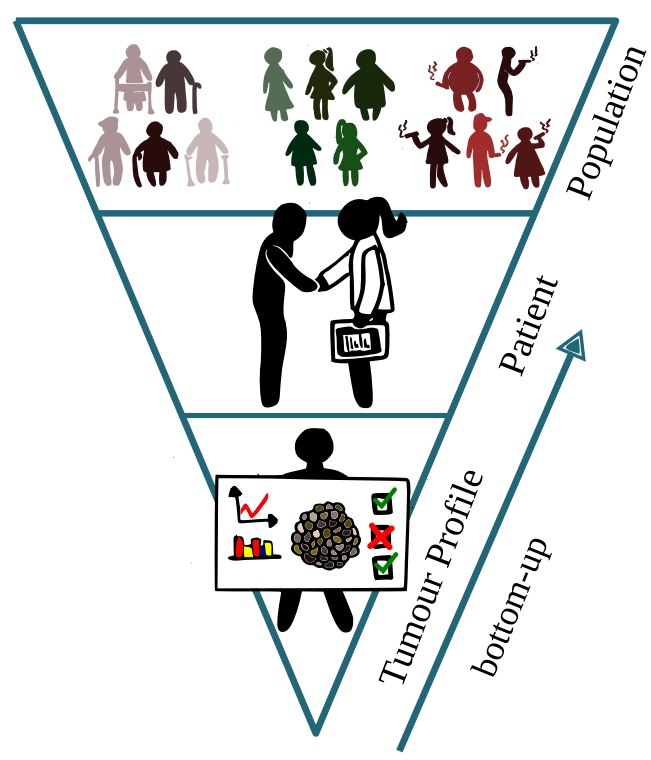

Figure 1.1: Personalised cancer practice on various levels, namely Population, Patient, Tumour Profile. On a population macro-level, a population can be categorised into various subpopulations (for instance based on age, gender or smoking habits), which in turn can be evaluated and risk-assessed for preventative, detective and corrective oncology practice. On a central, patient-specific level, cancer care may be tailored to fit the needs, lifestyle and priorities of the patient, in pursuit of medicine that optimises both treatment outcome and the patient's quality of life. On a tumour profile level, tumour-specific data can provide information which may contribute towards disease prognoses and intelligent treatment decisions. Narrowing down cancer care personalisation to tumour level allows for a bottomup approach to personalised tumour treatments.

The phrase bench-to-bedside describes the practice of transferring in vitro and in vivo findings from the laboratory to a clinical setting. Now, as the contemporary mathematician works on both blackboards and computer keyboards and we therefore, analogously, use the term blackboard-to-bedside to describe the action of translating mathematical and computational intelligence to clinical application, as is conceptually illustrated in Figure 1.2. Mathematical modelling has chronologically tailed clinical implementation of tumour treatment strategies. Historically, this time-lag is validated in the early era of modern cancer care practice, which preceded advanced technology. However, with current imaging and biopsy technologies, sophisticated in vitro and in vivo laboratories, accumulating data from experiments and clinics, available computational power [9] and biological, medical and mathematical knowledge, mathematical oncology to- 
day constitutes an up-to-date complement to traditional cancer research. Modelling has the potential to both optimise currently available anti-cancer protocols and contemporaneously aid preclinical developments of new anti-cancer therapies, thus the time-lag between clinical applications and mathematical modelling is conceptually being eliminated. In order to comprehensively transfer insights from blackboard-to-bedside, actualised collaborations between clinicians, biologists and mathematicians are key [49].

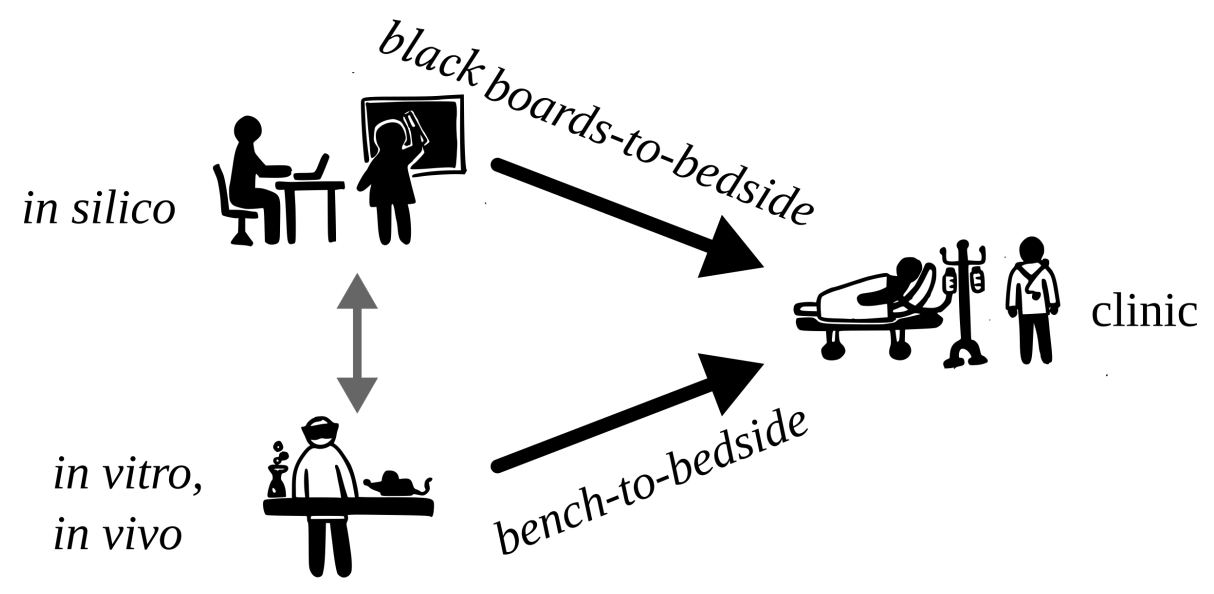

Figure 1.2: 'Bench-to-bedside' depicts the practice of transferring in vitro and in vivo findings from the laboratory to a clinical setting. The contemporary mathematician works on both blackboards and computer keyboards thus we therefore, analogously, present the term blackboard-to-bedside to describe the action of translating mathematical and computational intelligence to clinical application.

\subsection{Multiscale Models}

Cancer is a multiscale process in which subcellular mutations result in aberrations that are apparent on a cellular scale or on a tissue scale [50]. It follows that multiscale models may constitute an attractive choice for the mathematical oncologist, and throughout this thesis we will indeed simulate tumours using mathematical models that can be classified as multiscale, hybrid, agent-based and on-lattice. Multiscale models refer to models that incorporate information on multiple scales in space and/or time. By using multiscale models, we can 'zoom in' on details that we consider to be important, and we can 'zoom out' to get an overview of the full modelling scenario at hand [51]. When 


\section{INTRODUCTION}

modelling a solid tumour, for example, this entails that we can 'zoom in' and look at subcellular details in individual tumour cells, and then we can 'zoom out' again to see how the tumour is behaving as a whole. Hybrid models are models that incorporate multiple modelling techniques that can be regarded as contrasting. Such a model might integrate both mechanistic and phenomenological rules, or both continuous and discrete variables or both deterministic and stochastic phenomena. This can be very useful in the field of mathematical oncology, as biological and biochemical processes involved in cancer dynamics can be best expressed in various ways. Using a hybrid modelling technique, we can for example describe the concentration of a drug across a tumour as a continuous function using a mechanistic diffusion equation, whilst regarding cells as discrete entities [50]. Agent-based models include several agents that may interact with each other and their environment. Every agent is modelled individually and is thus distinct from other agents in the system [52]. In an agent based tumour model, for example, an agent can correspond to one cancer cell or a group of cancer cells. This naturally allows for heterogeneity amongst tumour cells, which is useful as tumour heterogeneity is associated with many implications in anti-cancer treatments (but more on that later on in the thesis). Combining agent-based models with multiscale and hybrid modelling approaches allows us to have environmental factors influence the behaviour and fate of agents. On-lattice models refer to models that play out on a lattice [50]. In an agent-based on-lattice model, for example, the movement of all agents is restricted to the lattice points. A cellular automaton $(\mathrm{CA})$ is a specific type of on-lattice model that allows a system, containing information about both agents and their environment, to evolve in time and space. In a CA, the quantity of any scalar field across the CA lattice is discretised to have a certain value on a certain lattice point. On-lattice models have the advantage of being easy to implement when coding for in silico experiments. In the following chapter, we describe the mathematical framework used throughout this thesis. This framework is based on a multiscale, hybrid cellular automaton.

Many solid tumours are derived from one cancerous seeding cell, which by detection time has produced a tumour with subclonal diversity displaying a few dominant subclones [53]. It has been observed that cells collected from the same tumour may display different subclonal [53] and spatio-temporal features influenced by intracellular, extracellular and intercellular mechanisms [54]. Consequently, a multitude of tumour metrics will 
vary within a tumour mass [46] and, what is more, this diversity may not be captured by current diagnostic tools [53]. However, in silico experiments provide a platform on which to conveniently study implications of spatio-temporal heterogeneities within a tumour, thus allowing us to observe what is not empirically feasible by other methods. One strength of multiscale, hybrid cellular automata, in particular, is their inherent capability to handle cellular, intratumoural variations, providing an opportunity to conveniently study the effects and implications of these spatio-temporal hetereogenities within a tumour. Accordingly, several multiscale models have recently been developed in order to fully capture the spatio-temporal, multiscale nature of tumour dynamics [6, 55-57]. Such models allow for intratumoural cross-scale integration of intracellular, extracellular and intercellular concepts, providing comprehensive modelling frameworks to which new biomedical information can easily be added. 
1. INTRODUCTION 


\section{Chapter 2}

\section{Mathematical Framework}

In this thesis, a multiscale mathematical framework is used to capture the multiscale nature of cancer. The framework, that is introduced in this chapter, is applied in the studies described in Chapters 3, 4 and 5.

\subsection{The Cellular Automaton}

The mathematical framework used throughout this thesis is, at its core, multiscale, hybrid cellular automaton (CA) in which a cancer cell population, or a tumour, is simulated on a square lattice that extends in two or three dimensions in space. Cellular automata organically enable spatio-temporal dynamics and intratumoural heterogeneity. Taking an agent-based approach, each cell (or group of cells) is modelled as an individual agent with individually computed intracellular features. Extracellular mechanisms such as oxygen and drug delivery across the lattice are regulated by mechanistic partial differential equations. Using well-formulated modelling rules, intracellular, extracellular and intercellular mechanisms can be integrated in the mathematical framework. A lattice point in the CA may be occupied by an agent (i.e. a cancer cell or a group of cancer cells) or an environmental feature such as extracellular solution (in vitro), or a blood vessel or extracellular space (ECM) (in vivo). Although the ECM comprises multiple components, such as collagen, elastin and fibronectin, we here make a well-established [58], simplifying modelling choice to not distinguish between these components. In our cellular automaton, a lattice point can at most be occupied by one agent, and thus the spatial step-size on the lattice is chosen to correspond to the size 


\section{MATHEMATICAL FRAMEWORK}

of an agent, corresponding to the smallest entity on the lattice. For example, when one agent corresponds to one cancer cell, then the spatial step size $(\Delta x)$ corresponds to the cell diameter. The time-step progression of the cellular automaton $(\Delta t)$ is defined by the event of shortest length in the modelling scenario at hand. Note that, if any numerical methods are incorporated in the modelling scenario at hand, $(\Delta x)$ and $(\Delta t)$ and must ensure that the solutions computed by the numerical methods are stable.

\subsection{Cell Cycle Models}

The cell cycle is the process that drives the duplication of cells and, by extension, the growth of a cancer cell population or a tumour. The cell cycle mechanism can be partitioned into four sequential phases, namely the gap 1 (G1), synthesis (S), gap 2 (G2) and mitosis (M) phase. A cell's DNA duplicates in the synthesis phase and chromosome segregation and cell division occur in the mitosis phase [59. The mitoses phase ends with the cell duplicating. In order to replicate its DNA and divide, a cell requires time to grow and double its protein and organelle mass between synthesis and mitoses. This happens in the intermediate gap phases G1 and G2. If some extracellular or intracellular conditions are unfavourable for cell cycle progression, a cell may delay its reproduction progress by (reversibly) exiting the G1 phase of the cell cycle to enter an inactive, quiescent phase (G0) [6]. Note that although quiescent cells are not cycling, they are not completely inactive, in fact they require energy to actively control their interior state [7].

A cell's current cycle phase significantly impacts its treatment responses to certain drugs and radiotherapy, as discussed later on in this chapter. Therefore, cell cycle details are included in our agent-based mathematical framework and the cell cycle progression for each agent (e.g. cancer cell) is tracked. There exists a number of mathematical cell cycle models to choose from, some of which are mechanistic and some of which are phenomenological, some of which are deterministic and some of which are stochastic. The modeller must decide which cell cycle model is the most appropriate to use for the modelling scenario at hand. Factors that influence the choice of cell cycle model include: goals and hypotheses of the experiment, desired level of details in the model, availability of data and allowed complexity in the mathematical and computational framework. In this thesis, three different types of cycle models are used. Specifically, 
in Chapter 3 we use a mechanistic cell cycle model described by a system of ordinary differential equations, in Chapter 4 we use a phenomenological model driven by a cell cycle clock and in Chapter 5 we use a stochastic model in which a cell progresses through the cell cycle via various cell cycle states (represented as nodes) connected by stochastic paths. Common for all three of these models is that they include some stochastic parameter, individually selected for each agent, that ensures that all cells do not cycle synchronously. This allows for cell cycle phase heterogeneity amongst cells. Also common for the three cell cycle models is that a cell's potential (and reversible) exit from the G1 phase of the cell cycle into the G0 state is based on extracellular conditions as explained in Section 2.3, and is thus not intrinsically described by the mathematical expressions used to compute cell cycle progression.

\subsubsection{A Mechanistic Cell Cycle Model}

In the mechanistic cell cycle model used in Chapter 3 in this thesis, the cell cycle is governed by a regulatory molecular network described by a system of ordinary differential equations (ODEs) (Equation 2.1] [6] in which the dependent variables are five different protein concentrations and cell mass (mass). The five proteins are namely the Cdkcyclin B complex $(C y c B)$, the APC-Cdh1 complex $(C d h 1)$, the p55cdc-APC complex in its total form $\left(p 55 c d c_{T}\right)$, the p55cdc-APC complex in its active form $\left(p 55 c d c_{A}\right)$ and the Plk1 protein in its active form $(P l k 1)$. The ODE that regulates the cell cycle reads

$$
\begin{aligned}
\frac{d[C y c B]}{d t} & =k_{1}-\left(k_{2}^{\prime}+k_{2}^{\prime \prime}[C d h 1]+[p 27 / p 21][H I F]\right)[C y c B] \\
\frac{d[C d h 1]}{d t} & =\frac{\left(k_{3}^{\prime}+k_{3}^{\prime \prime}\left[p 55 c d c_{A}\right]\right)(1-[C d h 1])}{J_{3}+1-[C d h 1]}-\frac{k_{4}[\text { mass }][C y c B][C d h 1]}{J_{4}+[C d h 1]} \\
\frac{d\left[p 55 c d c_{T}\right]}{d t} & =k_{5}^{\prime}+k_{5}^{\prime \prime} \frac{([C y c B][\text { mass }])^{n}}{J_{5}^{n}+([C y c B][\text { mass }])^{n}}-k_{6}\left[p 55 c d c_{T}\right] \\
\frac{d\left[p 55 c d c_{A}\right]}{d t} & =\frac{k_{7}[P l k 1]\left(\left[p 55 c d c_{T}\right]-\left[p 55 c d c_{A}\right]\right)}{J_{7}+\left[p 55 c d c_{T}\right]-\left[p 55 c d c_{A}\right]}-\frac{k_{8}[M a d]\left[p 55 c d c_{A}\right]}{J_{8}+\left[p 55 c d c_{A}\right]}-k_{6}\left[p 55 c d c_{A}\right] \\
\frac{d[P l k 1]}{d t} & =k_{9}[\text { mass }][C y c B](1-[P l k 1])-k_{10}[P l k 1] \\
\frac{d[m a s s]}{d t} & =\mu[\text { mass }]\left(1-\frac{[m a s s]}{m_{*}}\right)
\end{aligned}
$$




\section{MATHEMATICAL FRAMEWORK}

where the growth rate coefficient $\mu$ is stochastically picked for each individual cell in order to enable heterogeneous cell cycle lengths amongst cells [6]. The binary variable $[H I F]$ (occurring in Equation 2.1 a) represents the hypoxia inducible transcription factor-1 (HIF-1) pathway. This factor is only activated if the cell is classified as hypoxic [6], hence $[H I F]=1$ in hypoxic cells and $[H I F]=0$ in normoxic cells. Oxygen distribution, and by extension hypoxia, is governed by extracellular regulations as is described in Section 2.4, thus the system of ODEs integrates intracellular dynamics with extracellular dynamics. The variables and parameters in Equation 2.1 are listed in Table 2.1, and are chosen to be appropriate for mammalian cell lines, specifically cancer cells with a doubling time of around 25 hours [6].

In the mathematical model used in Chapter 3, a cancer cell is categorised as being in either the G1 phase or in the collective S-G2-M phase of the cell cycle and the Cyclin $\mathrm{B}$ concentration of a cell is used as a marker to determine cell cycle progression. A cell leaves the G1 state to enter the collective S-G2-M state when $[C y c B]$ increases, i.e. when $[C y c B](t+\Delta t)>[C y c B](t)$, where $t$ denotes time and $\Delta t$ is the time step. Cell division occurs when the cell exits S-G2-M phase to enter the G1 phase, and this happens when the threshold value $[C y c B]_{t h r}=0.1$ is crossed from above [6, 60]. Post cell division, the mass of the cell is halved, simulating one cell dividing into two cells [60.

\begin{tabular}{ll|l}
\hline Component & Rate constants $\left(\mathrm{h}^{-1}\right)$ & Dimensionless parameters \\
\hline$[\mathrm{CycB}]$ & $k_{1}=0.12, k_{2}^{\prime}=0.12$ & {$[H I F 1]=\left\{\begin{array}{cc}1 & \text { if } \hat{K} \leq 0.1, \\
0 & \text { otherwise }\end{array}\right.$} \\
& $k_{2}^{\prime \prime}=4.5,[\mathrm{p} 27 / \mathrm{p} 21]=1.05$ & \\
{$[\mathrm{Cdh} 1]$} & $k_{3}^{\prime}=3, k_{3}^{\prime \prime}=30, k_{4}=105$ & $J_{3}=0.04, J_{4}=0.04$ \\
{$\left[\mathrm{p} 55 \mathrm{cdc}_{T}\right]$} & $k_{5}^{\prime}=0.015, k_{5}^{\prime \prime}=0.6, k_{6}=0.3$ & $J_{5}=0.3, n=4$ \\
{$\left[\mathrm{p} 55 \mathrm{cdc}_{A}\right]$} & $k_{7}=3, k_{8}=1.5$ & $J_{7}=0.001, J_{8}=0.001,[\mathrm{Mad}]=1$ \\
{$[\mathrm{Plk} 1]$} & $k_{9}=0.3, k_{10}=0.06$ & $\epsilon$ is randomised, $\epsilon \in[-1,1]$ \\
{$[\mathrm{mass}]$} & $\mu=\mu^{+}+\epsilon \hat{\mu}$ & \\
\hline
\end{tabular}

Table 2.1: Parameters for the nondimensionalised form of Equation 1. As described in Section $2.4 \hat{K}$ denotes oxygenation value.

The value $m_{*}$ occurring in Equation (1f) denotes the maximum mass that a cell may reach, should it not be allowed to divide for some biological reason. Follow- 
ing previous work by Tyson and Novak [60], $m_{*} \gg \max ([\operatorname{mass}](x, t))$, specifically $m_{*}=10 \cdot[$ mass $]\left(x_{n b}, t_{n b}\right)$, where $[$ mass $]\left(x_{n b}, t_{n b}\right)$ corresponds to the mass of a "newborn" cell. The average growth rate constant $\mu^{+}=25$ hours and $\hat{\mu}=3$ hours are here chosen to produce cell cycle lengths between 22 hours and 28 hours for cells that are well oxygenated, i.e. cells that have the $[H I F]$ component occurring in Equation 2.1 switched off (i.e. set to zero). The $[H I F]$ component is activated (set to one) in hypoxic cells, this activation delays the cell cycle progression and yields a cell cycle length increase of approximately $20 \%$.

The cell cycle model here discussed is based on previous work by Tyson and Novak, who produced a series of papers describing the underlying mechanisms of cell cycle progression and cell cycle regulation in mathematical terms [60-62]. By identifying key proteins involved in controlling and driving the cell cycle, they managed to condense the complex biological process, that is the cell cycle, into a six-component regulatory molecular network [60]. The opposing and oscillating nature between Cyclin-dependent protein kinases (Cdks) and the anaphase-promoting complex (APC) plays a central role in cell cycle regulation and yields a hysteresis feedback loop. Cyclin $\mathrm{B}([\mathrm{CycB}])$ is part of the Cdk family whilst Cdh1 is part of the APC. As is demonstrated in Figure 2.13, the cell cycle control system consists of two steady states, namely the G1-phase state and the S-G2-M-phase state. $[C y c B]$ is low, whilst $[C d h 1]$ is high in the G1 state and, conversely, $[C y c B]$ is high, whilst $[C d h 1]$ is low in the S-G2-M state. Other auxiliary molecules are included in the model to enable appropriate lagging transitions between these two steady states. Auxiliary molecule concentrations over time are illustrated in Figure 2.1p. At the start of a cell cycle, the system will tend towards a G1 steady state and at the end of the cell cycle the system will tend towards a S-G2-M steady state. Details are provided by Tyson and Novak [60]. As shown in Figure 2.1, cell mass, [mass], doubles over the course of a cell cycle and is later reset, i.e halved, at cell division.

Descriptive remarks regarding each equation occurring in the system of ordinary differential equations (Equation 2.1), used to model cell cycle progression are listed below. 
(a)

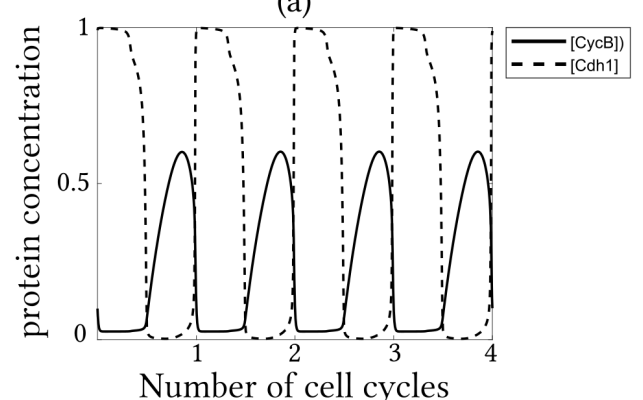

(c)

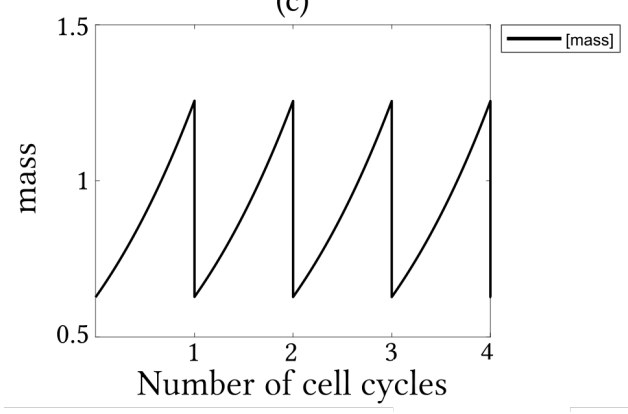

(b)

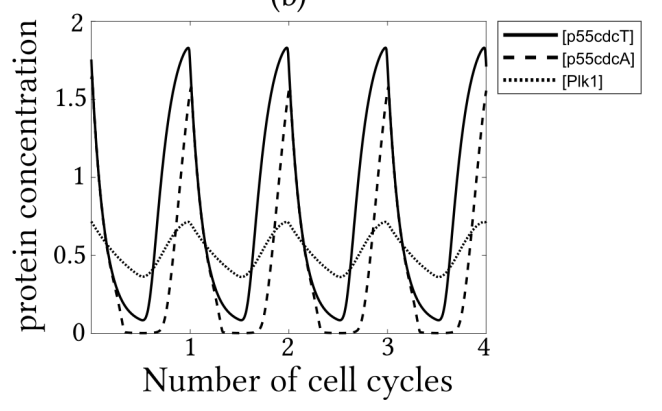

Figure 2.1: Time evolution of protein concentrations in one simulated cancer cell (measured in grams of protein per gram of total cell mass), where the cell cycle length is 25 hours. (a) The opposing dynamics between $[C y c B]$ and $[C d h 1]$, which is key in the used mathematical cell cycle model. (b) Concentrations of the auxiliary proteins $[P k l 1]$ and p55cdc in its total form $\left[p 55 c d c_{T}\right]$, and its active form $\left[p 55 c d c_{A}\right]$. (c) Cell mass, [mass] over time, where $[$ mass $]$ is halved at the very start of each cell cycle, i.e. immediately after cell division. 
- Remarks regarding Equation (2.17):

$\frac{d[C y c B]}{d t}=k_{1}-\left(k_{2}^{\prime}+k_{2}^{\prime \prime}[C d h 1]+[p 27 / p 21][H I F]\right)[C y c B]$.

Cdks are necessary for DNA replication, and accordingly Cdk activity is high in the S-G2-M state. Conversely, Cdk activity is low in the G1 state. The rate of change of the $\mathrm{Cdk}[C y c B]$ is governed by synthesis and reduction, where the reduction is considered to be partly independent of other molecules in the system, and partly induced by the presence of [Cdh1] and the two proteins $p 21$ and $p 27 . p 21$ and $p 27$ are known to inhibit Cdks, they are here merely treated as parameters via the factor $[p 27 / p 21]$ that is activated in hypoxic cells only via the $[H I F]$ component [6].

- Remarks regarding Equation (2.1p) :

$$
\frac{d[C d h 1]}{d t}=\frac{\left(k_{3}^{\prime}+k_{3}^{\prime \prime}\left[p 55 c d c_{A}\right]\right)(1-[C d h 1])}{J_{3}+1-[C d h 1]}-\frac{k_{4}[\text { mass }][C y c B][C d h 1]}{J_{4}+[C d h 1]} .
$$

The p55cdc-APC in its total $\left(p 55 c t c_{T}\right)$ and active form $\left(p 55 c d c_{A}\right)$ is also part of the APC. The $[C d h 1]$ time derivative obeys Michaelis-Menten type equations, where the occurring J-constants are Michaelis constants. Here, $[C y c B]$ inhibits $[C d h 1]$ activity whilst $\left[p 55 c d c_{A}\right]$ promotes it. The $[C y c B]$-derived inactivation of $[C d h 1]$ is assumed to occur in the cell nucleus, hence $[C y c B]$ will accumulate in the nucleus and it follows that $[C y c B]$ will increase with cell mass, thus the $[$ mass] factor is incorporated in the second term that describes the suppressing effect that $[C y c B]$ has on $[C d h 1]$. For "newborn" cells, $[$ mass $]$ is minimal, however as the cell cycle progresses, $[$ mass $]$ increases, consequently promoting $[C y c B]$ whilst demoting $[C d h 1]$.

- Remarks regarding Equation (2.1) :

$$
\frac{d\left[p 55 c d c_{T}\right]}{d t}=k_{5}^{\prime}+k_{5}^{\prime \prime} \frac{([C y c B][\text { mass }])^{n}}{J_{5}^{n}+([\text { CycB }][\text { mass }])^{n}}-k_{6}\left[p 55 c d c_{T}\right] .
$$




\section{MATHEMATICAL FRAMEWORK}

$\left[p 55 c d c_{T}\right]$ syntheses occurs naturally throughout the cell cycle but it is also synthesised in the S-G2-M phase by $[C y c B]$, as is appropriately described by a Hill function. However, p55cdc is not active once newly synthesised, and instead this activation is described in Equation (1d).

- Remarks regarding Equation (2.1 d) :

$$
\frac{d\left[p 55 c d c_{A}\right]}{d t}=\frac{k_{7}[P l k 1]\left(\left[p 55 c d c_{T}\right]-\left[p 55 c d c_{A}\right]\right)}{J_{7}+\left[p 55 c d c_{T}\right]-\left[p 55 c d c_{A}\right]}-\frac{k_{8}[M a d]\left[p 55 c d c_{A}\right]}{J_{8}+\left[p 55 c d c_{A}\right]}-k_{6}\left[p 55 c d c_{A}\right] .
$$

In the model, $[P l k 1]$ is included to transform $\mathrm{p} 55 \mathrm{cdc}$ into its active form $\left[p 55 c d c_{A}\right]$. Tyson and Novak [60] describe $[P l k 1]$ as a hypothetical enzyme driving $\left[p 55 c d c_{T}\right]$ activation, and this effect is incorporated with Michaelis-Menten equations. Furthermore, $[\mathrm{Mad}]$ represents a family of checkpoint genes, here treated as a parameter, which are able to deactivate $\left[p 55 c d c_{A}\right]$, should DNA synthesis or chromosomes alignment not be completed rapidly enough to allow correct cell cycle advancement.

Remarks regarding Equation (2.1p) :

$\frac{d[P l k 1]}{d t}=k_{9}[$ mass $][C y c B](1-[P l k 1])-k_{10}[P l k 1]$

$[P l k 1]$ decreases naturally whilst $[C y c B]$ enhances $[P l k]$ activity. It is here, again, apparent that the $[$ mass $]$ component boosts effects of $[C y c B]$.

- Remarks regarding Equation (2.1f):

2.1]) $\frac{d[\text { mass }]}{d t}=\mu[$ mass $]\left(1-\frac{[\text { mass }]}{m_{*}}\right)$.

The dependent variable [mass] will double over the course of one cell cycle, following an adapted logistic equation. 


\subsubsection{A Phenomenological Cell Cycle Clock Model}

In the phenomenological cell cycle model used in Chapter 4, progression through the cell cycle is governed by a cell cycle clock. Thus in our agent-based framework, every agent is attributed an individual clock that drives and tracks cell cycle progression. To achieve asynchronous cycling amongst cancer cells, every cell $i$ is assigned an individual, stochastic doubling-time $\tau_{i}$, corresponding to the time it takes for a cell to complete one cell cycle under well-oxygenated conditions. Specifically, we here pick $\tau_{i}$ is from a normal distribution with a mean value $\mu$ and a standard deviation $\sigma$. The choice of using a normal distribution is motivated by the fact that cell duplication yields imperfect cell copies that are almost, but not perfectly, synchronised. The choice of distribution from which to pick $\tau_{i}$ may ideally be motivated by experimental data, and can be adjusted if appropriate.

The fraction of time spent in each of the four cell cycle phases G1, S, G2 and M in well-oxygenated cells are respectively denoted $\Theta_{G 1}, \Theta_{S}, \Theta_{G 2}$ and $\Theta_{M}$. The $\Theta$-fractions sum up to one so that

$$
\Theta_{G 1}+\Theta_{S}+\Theta_{G 2}+\Theta_{M}=1
$$

The values $\Theta_{j}$, for $j=\mathrm{G} 1, \mathrm{~S}, \mathrm{G} 2, \mathrm{M}$ are here approximations gathered from literature to represent typical lengths of rapidly cycling human body cells, with a doubling time of roughly 24 hours, and respectively have the values $11 / 24,8 / 24,3 / 24$ and $1 / 24$ [63]. Thus, under well oxygenated conditions, the total amount of time that agent $i$ spends in cell cycle phase $j$ becomes $\Theta_{j} \cdot \tau_{i}$. Low cellular oxygenation values have, however, been shown to stall cell cycle progression by inducing G1 arrest in particular [1]. Recall that in the mechanistic cell cycle model presented in Section 2.2.1, the G1 phase is inherently elongated under hypoxic conditions [6]. For this phenomenological clock-type model, however, there is no mechanistic functionality driving G1-arrest under hypoxic conditions so, we therefore introduce an auxiliary function to achieve oxygen-dependent 


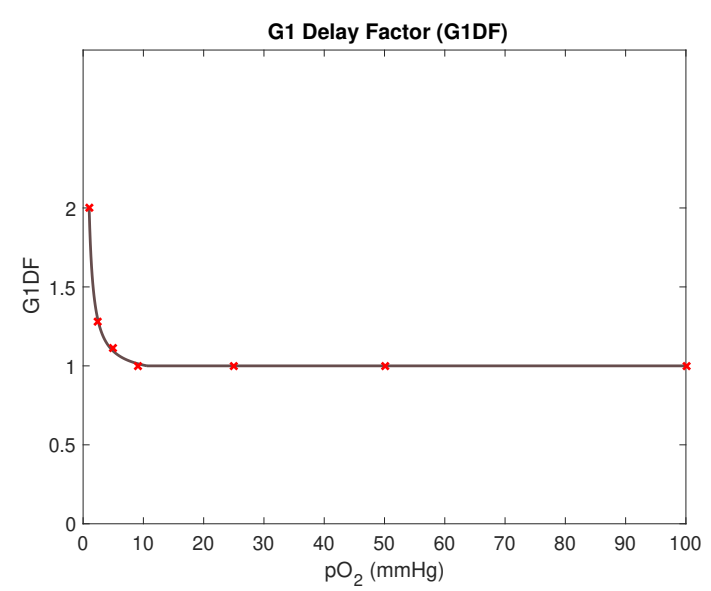

Figure 2.2: The G1 Delay Factor (G1DF) is incorporated in the model to achieve oxygendependent G1 arrest. The G1DF (dark line) is approximated using results (red crosses) from a previous mathematical study by Alarcon et al. [1].

G1 arrest. We call this function the G1 Delay Factor $(G 1 D F)$ where,

$$
G 1 D F(\hat{K}(x, t))= \begin{cases}2 & \text { if } 0 \% \leq \hat{K}(x, t)<1 \% \\ a_{1}+\frac{a_{2}}{a_{3}+\hat{K}(\bar{x}, t)} & \text { if } 1 \% \leq \hat{K}(x, t) \leq 10.5 \% \\ 1 & \text { otherwise. }\end{cases}
$$

and $\hat{K}$ here denotes the oxygenation (in units of $\mathrm{mmHg}$ ). The $G 1 D F$ approximates how much the G1 phase is expanded as a function of oxygenation.

As illustrated in Figure 2.2, the piece-wise function G1DF is an approximation that is chosen to match data points extracted from a mathematical study by Alarcon et al. [1], in which a mechanistic Tyson-Novak cell cycle model is extended to incorporate the action of the p27 protein which delays cell cycle progression and is upregulated under hypoxia. The time that agent $i$ spends in the $G_{1}$ phase is thus now given by $G 1 D F(\hat{K}) \cdot \Theta_{G 1} \tau_{i}$. Lengths of other cell cycle phases are here modeled to be nonoxygen dependent.

\subsubsection{A Stochastic Cell Cycle Clock Model}

Biological systems and mechanisms inherently display a level of stochasticity, the cell cycle included [5, 64]. As thoroughly described in Chapter 5, and pictorially illustrated in Figure 5.2, cancer cells are correlated with high replication stress and thus during 
the cell cycle, some cancer cells will need to repair themselves before progressing to subsequent events in the cell cycle [5]. To account for the fact that not every cell will progress though the cell cycle the same way, the cell cycle can be modelled as consisting of several sub-events represented by nodes in a graph, and between the nodes are several paths, or edges. Building on the cell cycle model describes in Section 2.2.2, a cell cycle clock here determines when a cell progresses to a subsequent cell cycle state, and a stochastic 'dice roll' determines which path the cell takes. A stochastic cell cycle model approach is useful when we want to incorporate cell cycle sub-events that some, but not all, cells experience.

\subsection{Cell Division and Tumour Growth}

In our framework, when a cell has completed the mitoses $(\mathrm{M})$ phase of its cell cycle, it duplicates and produces a secondary cell, namely a daughter cell, on a random lattice point in its spherical neighbourhood. In order to achieve approximately spherical-like tumour growth on a square lattice, the model stochastically alternates between placing daughter cells in Moore and von Neumann neighbourhoods [6]. The 1st, 2nd and 3rd order neighbourhood (O.N.) of a parental agent using (a) the von Neumann convention and (b) the Moore convention are illustrated in Figure 2.3. A daughter cell is allowed to be placed on up to $\nu$ order neighbourhoods of its mother cell, where lower order neighbourhoods are strictly prioritised. Thus the first order neighbourhood must be filled before cells can be placed in the second order neighbourhood and so on. The value of $\nu$ depends on the modelling scenario at hand. When simulating in vitro monolayer cell cultures, there is often no spatial constraint or lack of nutrients that would hinder a cell from dividing, in which case $\nu$ can be set to Infinity, or half of the lattice size to ensures that the cells are restricted to the simulated lattice. In vitro cell spheroids and in vivo tumours, however, often consist of a quiescent core with G0 cells enclosed by a shell of proliferating cells [3, 5]. This heterogeneity of cycling and non-cycling cells can be replicated in our model by only allowing daughter cells to be placed on up to $\nu$ order neighbourhoods, where $\nu$ is finite and can, ideally, be calibrated from experimental data. A G1 cell that is not allowed to divide, due to the lack of space within its $\nu$ th order neighbourhood, is set to be in state G0 [6]. This is a reversible 


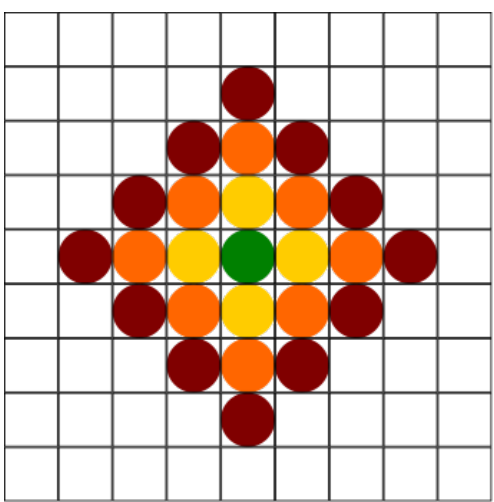

(a) von Neumann neighbourhoods

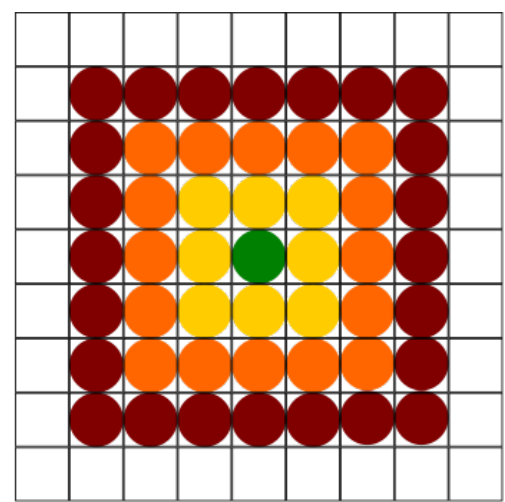

(b) Moore neighbourhoods

\section{Parent \\ 1st O.N. \\ 2nd O.N. \\ 3rd O.N.}

Figure 2.3: The 1st, 2nd and 3rd order neighbourhood (O.N.) of a parental agent using (a) the von Neumann convention and (b) the Moore convention.

process, as a G0 cell may re-enter the G1 phase of the cell cycle should space become available again as a result of, for example, anti-cancer targeting.

\subsection{Oxygen Dynamics}

Solid tumours typically contain a hypoxic core [7]. Hypoxia occurring in solid tumours can broadly be categorised into two subtypes, namely chronic hypoxia and transient hypoxia. Chronic hypoxia occurs in the microenvironment as a result of permanent diffusion limitations, whilst transient hypoxia is perfusion limited and brought on by interim oxygen shortages caused by temporary shut downs of blood vessels or alterations in oxygen flux [65]. Cells experiencing hypoxia may alter their protein synthesis in order to adapt to low oxygen conditions and conserve energy, resulting in reduced proliferation rates [66]. Moreover, radiotherapy responses are reduced in hypoxic tumour regions as later discussed in Section 2.6.2. Due to the prevalence and implications of intratumoural hypoxia, oxygen dynamics are included in our mathematical framework in which the distribution of oxygen across the CA lattice is modelled using a mechanistic partial differential equation (PDE). Oxygen is produced on lattice points that are labeled as oxygen source points. Depending on the model scenario at hand, these oxygen source points can for example be blood vessels or lattice boundaries. In our mathematical framework, the oxygen concentration $K(x, t)$ in lattice point $x$ at time $t$ is computed 
using

$$
\frac{\partial K(x, t)}{\partial t}=\nabla \cdot\left(D_{K}(x, t) \nabla K(x, t)\right)+r_{K} m(x, t)-\phi_{K} K(x, t) \operatorname{cell}(x, t),
$$

coupled with no-flux boundary conditions. $D_{K}(x, t)$ here denotes the oxygen diffusion coefficient which is higher in lattice points occupied by cells than in unoccupied lattice points [6]. The function $\operatorname{cell}(x, t)$ is binary and equal to one if the lattice point is occupied by a cancer cell, and zero otherwise. Similarly, the binary function $m(x, t)$ is one if the lattice point $x$ is an oxygen source point at time $t$, and zero otherwise. Furthermore, $r_{K}$ is the oxygen production coefficient and $\phi_{K}$ denotes cellular oxygen consumption. Hence the first term in Equation 2.4 describes oxygen diffusion, the second term describes oxygen production and the third term describes oxygen consumption.

The no-flux boundary conditions, and the non-fixed oxygen production and consumption rates, will cause the total amount of oxygen on the lattice to fluctuate over time. In order to express the oxygenation levels of lattice points in units of $\mathrm{mmHg}$, we introduce a scaled oxygen variable $\hat{K}(x, t)$, such that

$$
\hat{K}(x, t)=\frac{K(x, t)}{\max _{n} K(n, t)} \cdot h,
$$

where $\max _{n} K(n, t)$ denotes the maximal $K(x, t)$-value, out occurring on the lattice at time $t$ [67] and $h$ is a scaling factor that should ideally be calibrated by experimental data. If a cell has a scaled oxygen value such that $\hat{K}(x, t) \leq 10 \mathrm{mmHg}$, it is classified as hypoxic [6]. In the mathematical framework, the $\hat{K}(x, t)$-value influences G1-arrest and responses to radiotherapy amongst other things.

\subsection{Drug Dynamics}

\subsubsection{Some Basic Concepts in Pharmacology}

Pharmacology can be described as 'The study of how drugs affect functionalities of living systems' and, in turn, a drug can be defined as substance (that is not a nutrient nor an essential dietary constituent) of which the chemical structure is known, that induces 


\section{MATHEMATICAL FRAMEWORK}

a biological effect in a living organism once said living organism is subjected to the drug [7, 68, 69]. Drug chemicals can be either synthetic or natural, i.e. man-made or obtained from flora or fauna. Moreover, a medicine has the purpose of achieving some therapeutic effect and may (but does not necessarily) contain one or multiple drugs, often in combination with other substances such as solvents and stabilisers that facilitate medicine administration. In order to achieve pharmacological responses, drug molecules must chemically influence one or more cell constituents. In other words, drug molecules need to bind to these cell constituents, which are often referred to as 'drug targets' or just 'targets', in order for pharmacological responses to be achieved in a biological system [7]. The most common targets include receptors and enzymes, however many anti-tumour drugs directly target the DNA. Receptors are protein molecules that respond to endogenous chemical signals, and enzymes are protein molecules that catalyse chemical reactions without getting consumed in the process [68].

When drugs are administered to a living organism, the number of molecules in the organism significantly exceeds the number of drug molecules [7]. This means that if the drug molecules were to be randomly distributed across the living organism as a whole, the chance of drug molecules binding to particular targets would be minimal. Drugs must therefore be able to somehow target specific cells or tissues in the body in order to be useful in therapeutic settings. The binding site specificity of a drug describes how good the drug is at selectively binding to its intended targets, without binding elsewhere [7, 68]. Complete binding site specificity can, however, not be achieved in practice. Drug binding to unintended targets may induce unwanted biological effects that can manifest as side effects in therapeutic settings. Drugs with low potency are often administered in higher doses, and this increases the likelihood of drug molecules binding to unintended targets. If a target (such as a receptor) is occupied by a drug molecule, this may, but does necessarily, alter the behavior of the receptor. If the target behaviour is indeed altered, we say that the target is activated. Note that drug binding and drug activation are two separate steps involved in the process of drugs producing biological effects. The affinity of a drug describes the drug's tendency to bind to targets whilst the efficacy describes the tendency of a bound drug molecule to activate targets [7, 68]. A drug can be classified as a, full or partial, agonists or an antagonists. For an agonist, the drug molecules bind to targets and, in most cases, activate biological responses. On the 
other hand, for an antagonist, the drug molecules bind to target receptors but do not activate biological responses [7, 68]. A drug's potency depends both on its affinity and efficacy, but in most cases highly potent drugs have high affinity. Full agonists have maximal efficacy and partial agonists have high (but not maximal) efficacy whilst full antagonists have negligible (zero) efficacy. The agonist/antagonist classification can be conceptually understood by the below schematics,

$$
\begin{aligned}
& \text { Agonist: } \mathrm{A}+\mathrm{R} \underset{\mathrm{k}_{-1}}{\stackrel{\mathrm{k}_{+1}}{\rightleftharpoons}} \mathrm{AR} \underset{\beta}{\stackrel{\alpha}{\rightleftharpoons}} \mathrm{AR}^{*} \rightarrow \text { Response, } \\
& \text { Antagonist: } \mathrm{A}+\mathrm{R} \underset{\mathrm{k}_{-1}}{\stackrel{\mathrm{k}_{+1}}{\rightleftharpoons}} \mathrm{AR} \rightarrow \text { No Response, }
\end{aligned}
$$

where A denotes a ligand (i.e. any chemical, such as a drug, that binds to receptors), $\mathrm{R}$ denotes target receptors and $\mathrm{A}$ binds to $\mathrm{R}$ to form the complex AR [7].

\subsubsection{Drug Binding and Drug Activation}

If ligand $\mathrm{A}$ reacts with receptors $\mathrm{R}$ to form a complex $\mathrm{AR}$, then the reaction can be described by

$$
\mathrm{A}+\mathrm{R} \underset{\mathrm{k}_{-1}}{\stackrel{\mathrm{k}_{+1}}{\rightleftharpoons}} \mathrm{AR} \text {. }
$$

Now, if we regard a system with a total number of target receptors $N_{t o t}$, of which $N_{A}$ receptors are occupied by molecules of ligand A, then there are $N_{t o t}-N_{A}$ free target receptors available in the system. More precisely, we here let $N_{A}$ correspond to the steady state number of occupied receptors, and we let $x_{A}$ denote the ligand concentration. If we assume that the number of added drug molecules $\left(N_{\bar{A}}\right)$, is much greater than the number of available target receptors in the system $\left(N_{t o t}\right)$, so that $N_{\bar{A}}>>N_{t o t}$, then the ligand concentration $x_{A}$ can assumed to be unaffected by the number of bound molecules, $N_{A}$. Hence $x_{A}$ can be assumed to be constant [7]. The Law of Mass Action dictates that the rate of a chemical reaction is proportional to the product of the concentrations of the substances that are reacting [7]. Using the law of 


\section{MATHEMATICAL FRAMEWORK}

mass action, the forward and backward reactions in Equation 2.6 can thus be obtained as

Forward reaction rate: $k_{+1}\left(x_{A} \cdot\left(N_{t o t}-N_{A}\right)\right)$,

Backward reaction rate: $k_{-1} N_{A}$.

At steady state there is an equilibrium, so the rate of the forward reaction must equal the rate of the backward reaction, hence,

$$
k_{+1}\left(x_{A} \cdot\left(N_{t o t}-N_{A}\right)\right)=k_{-1} N_{A} .
$$

From this, the affinity constant, defined as $k_{+1} / k_{-1}$, can be obtained as

$$
\frac{k_{+1}}{k_{-1}}=\frac{N_{A}}{x_{A} \cdot\left(N_{t o t}-N_{A}\right)}[l / m o l]
$$

The reciprocal of the affinity constant is referred to as the equilibrium dissociation constant, $K_{A}$, such that

$$
K_{A}=\frac{k_{-1}}{k_{+1}}=\frac{x_{A} \cdot\left(N_{t o t}-N_{A}\right)}{N_{A}}[\mathrm{~mol} / \mathrm{ll}]
$$

which depends on both the drug and the target receptor. Since the equilibrium dissociation constant is measured in units of concentrations (e.g. [mol/l]) it is more intuitive to use than the affinity constant. Further, the occupancy, $p_{A}$, describes the fraction of occupied target receptors, i.e. $N_{A} / N_{t o t}$, and can be obtained from Equation 2.9 so that

$$
p_{A}=\frac{N_{A}}{N_{t o t}}=\frac{N_{A}}{K_{A} N_{A} / x_{A}+N_{A}}=\frac{x_{A} / K_{A}}{1+x_{A} / K_{A}},
$$

this is the Hill-Langmurir equation that describes how the target occupancy is related to the drug concentration [7]. When plotting the occupancy $p_{A}\left(x_{A}\right)$ over a linearly increasing drug concentration $x_{A}$, the characteristic shape of a rectangular hyperbola is obtained. Alternatively, if the drug concentrations increases on a logarithmic scale, the $p_{A}\left(x_{A}\right)$-curve takes a sigmoidal shape [68, 170], as is demonstrated in Figure 2.4. If more than one drug is present, things get slightly more intricate but that is outside the scope of this thesis. From the above equation (Equation 2.10, it is clear that if $x_{A}=K_{A}$, then $p_{A}=0.5$. 


\subsection{Drug Dynamics}

Target occupancy over drug concentration
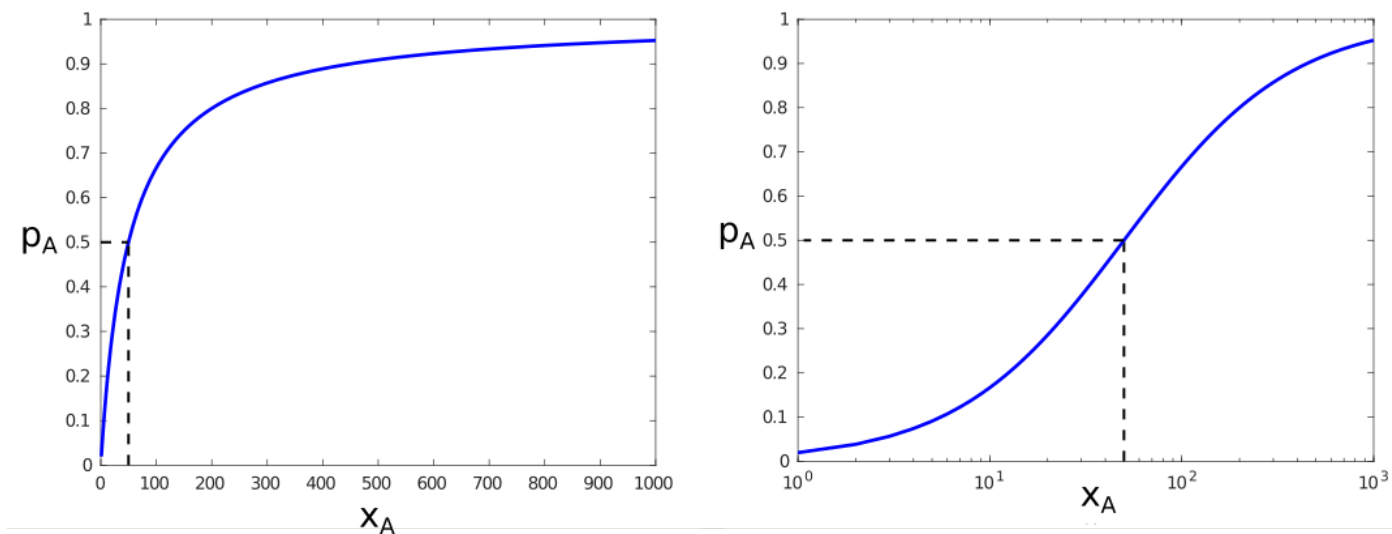

Figure 2.4: The target occupancy $\left(p_{A}\right)$ plotted over drug concentration $\left(x_{A}\right)$ when the drug concentration increases on a linear (left) and logarithmic (right) scale. Here $K_{A}$, i.e. the drug concentration that yields $50 \%$ target occupancy, is $x_{A}=100$ (concentration units).

Typical drug binding curves used in experimental settings graph the amount of drug bound over drug concentration, this can be directly measured using radioactive atoms [7. If we are instead interested in assessing the biological effect or response, not just the drug binding, then we can graph drug effect in [\%] over concentration (in vitro) or the drug response in [\%] over drug dose (in vivo). An effect or response of $100 \%$ corresponds to the maximal effect or response achievable by the drug, denoted $E_{\max } . E C_{50}$ and $E D_{50}$ respectively denote the drug concentration and drug dose required to achieve half of the maximum effect, i.e. $0.5 \cdot E_{\max }$. A modeller may quantitatively formulate what $E_{\max }$ corresponds to in the specific scenario at hand. Using the Hill-Langmurir Equation, and the simplifying assumption that drug effect is proportional to target occupancy, we arrive at the Emax model [70-72], a ubiquitous model in pharmacodynamics which expresses the in vitro relationship between the drug concentration $C$, and drug effect $E(C)$, as

$$
E(C)=E_{\max } \frac{C}{E C_{50}+C}
$$

In line with previous discussion, the Emax model achieves a sigmoidal drug response curve. To achieve steeper growth curves, the Emax model can be expanded to the sigmoidal Emax model [70], in which a coefficient (termed the hill-coefficient and here 


\section{MATHEMATICAL FRAMEWORK}

denoted gamma) is introduced so that

$$
E(C)=E_{\max } \frac{C^{\gamma}}{E C_{50}^{\gamma}+C^{\gamma}} .
$$

We can use the sigmoidal Emax model in our mathematical framework to compute how cells respond to local drug concentrations. If appropriate, a term $+E_{0}$ can be added on the right-hand side of Equation 2.12 to represent a base effect in the absence of drugs [73].

\subsubsection{Cancer Drugs}

Cancer cells are merely damaged, corrupted human body cells and consequently, this makes them more difficult to selectively target in vivo than for example bacteria, because microorganisms differ from healthy human body cells in ways that cancer cells, naturally, do not. Cancer drugs must, however, somehow be able to mainly target cancer cells, not all human body cells and thus we need to differentiate between cancer cells and healthy human body cells in some way. One way to make this differentiation is by noticing that cancer cells are relatively proliferative whilst most human body cells are in a non-cycling state. Many conventional anti-cancer drugs take advantage of this fact and thus target all cycling cells, or more specifically mechanisms involved in the cell cycle and cell division process [7, 74, 75]. The premise is that this will mainly attack cancer cells whilst keeping enough human body cells untargeted to validate drug administration [7, 76]. This explains why common chemotherapy side-effects are associated with the types of human body cells that are constantly dividing and cycling, such as hair cells associated with the side-effect alopecia. There exists multiple anti-cancer drugs, approximately 80 of which are used in British clinics, often in combination with other drugs or anti-cancer treatments [70]. In this thesis, we will investigate the effect of three, rather different, anti-cancer drugs in silico. In Chapter 3, we consider treatments with traditional chemotherapeutic drugs. In Chapter 4, we investigate the effects of hypoxia-activated prodrugs (HAPs), that act as 'Trojan Horse' drugs being, conceptually, harmless until they reach hypoxic (tumour) regions in which warheads are released. Finally, in Chapter 5 we study drugs that target cellular DNA-damage responses, and thus may hinder cells from repairing themselves post erroneous DNA replication. 


\subsubsection{Drug Disposition}

When drugs are administered in vivo, the stages involved in drug disposition are commonly categorised using the ADME acronym, where the letters stand for: Absorption of drugs from the drug administration site, Distribution of drugs within the body, Metabolism of drugs and Excretion of drugs [7, 69, 77]. In vivo, drug molecules are dispositioned via bulk flow and diffusion. Bulk flow describes the process in which the drugs are transported over long distances via, for example, the bloodstream or the cerebrospinal fluid. Over short distances, however, the drug molecules are transported via diffusion. The diffusion is highly affected by the chemical structure of the drug, whilst the bulk flow transportation is not [7]. The diffusion rate of a drug is predominately affected by the molecular size of the drug and, more specifically, the diffusion coefficient of a drug is inversely proportional to the square root of the molecular weight of the drug, so that large molecules diffuse more slowly than do small molecules [7]. In many cases, drugs can be assumed to be well stirred with a uniform drug distribution within some regarded body compartment, and it is the inter-body compartment drug movement that determines how long a drug will be present in the body post drug administration. Drug elimination describes the irreversible process of drug removal from the body. It is achieved by metabolism and excretion. Metabolism refers to the chemical build-up and break down of drugs, and excretion refers to elimination of drug from the body via some excretory route and elimination transporter, e.g. kidneys and urine [7]. In many cases when describing drug elimination, we can use first order kinetics which means that the rate of drug elimination is directly proportional to the drug concentration. First order kinetics results in exponential drug decay. As a comparison, zero order kinetics means that the drug is eliminated at a constant rate. In our mathematical framework, the drug concentration $C(x, t)$ in location $x$ at time $t$ is computed using a diffusion-reaction equation where

$$
\frac{\partial C(x, t)}{\partial t}=\nabla \cdot\left(D_{C}(x, t) \nabla C(x, t)\right)+p(x, t)-\eta_{C} C(x, t)
$$

and $D_{C}(x, t)$ denotes the diffusion coefficient, $p(x, t)$ is a supply term and $\eta_{C}$ is a first order drug elimination coefficient. 


\section{MATHEMATICAL FRAMEWORK}

\subsection{Radiotherapy}

\subsubsection{Effects of Radiation in Biological Materials}

Today, the majority of cancer patients receive radiotherapy in some form [78]. When biological materials are exposed to radiation, they absorb energy from the radiation. This energy absorption can cause an electron in some atom or molecule in the biological material to get excited to a higher energy level (excitation) or to get ejected from its atom or molecule (ionisation) [79]. Different types of radiation are being used in clinical settings, but in this work we will focus on X-rays, a form of electromagnetic, ionising radiation (IR). X-rays are produced in electrical devices using the kinetic energy from high energy electrons [79]. X-rays themselves do not damage cell targets (DNA molecules), but when energy from X-rays is absorbed by the biological material, this results in the production of fast-moving charged particles that do have the ability to damage cell targets [79]. IR-induced DNA damage can be grouped into two main categories: $(i)$ damage by direct action of radiation and (ii) damage by indirect action of radiation [79]. Direct damage occurs when an electron, that has been ejected from some atom or molecule in the biological material as a result of ionising radiation, directly interacts with DNA molecules. Indirect damage occurs when the ejected electron reacts with other molecules in the cell, that in turn interact with DNA molecules and thus indirectly cause DNA damage [80]. These 'other molecules' are most commonly water molecules, since cells consist of roughly $80 \%$ water [79]. When radiation interacts with water molecules in the cell, free radicals are formed, and the amount of free radicals formed is furthermore dose-dependent [81]. Free radicals are atoms or molecules that each have an unpaired valence electron, making them highly reactive and thus prone to reacting with (in this case) DNA molecules. Reactions between free radicals and cellular DNA may result in alterations of the molecular DNA structure, which may induce cell death or the impairment of some cell functions. It is estimated that two thirds of the total X-ray induced DNA damage is caused by indirect actions of radiation [79].

A DNA molecule famously consists of two strands, wrapped in double-helical struc-

ture, that are connected by bases. It is the sequence of the four occurring bases (adenine, guanine, thymine and cytosine) that stipulate the genetic code. Each strand is, furthermore, held together by sugar and phosphate groups. Radiation can produce multiple 
types of DNA lesions in cells, some of which are easy for (mammalian) cells to selfrepair, and some of which are not [79]. In the context of clinical radiotherapy, DNA strand breaks are the most impactful type of such DNA lesions [79]. They occur when the sugar-phosphate 'scaffolding' in the DNA strands gets damaged and disruptions in the DNA helix consequently occur. Two main types of strand breaks can take place: single-strand breaks (SSBs) and double-strand breaks (DSBs) [79]. SSBs occur when one of the strands in a local region of the DNA double-helix breaks, whilst DSBs occur when both of the strands in a local region of the DNA double-helix break. Note the word local here, as cells can handle two strand breaks occurring on opposite strands located more than approximately ten base pairs (or $4 \mathrm{~nm}$ ) apart as two SSBs [79]. Cells are better at repairing SSBs than they are at repairing DSBs, because post SSBs cells can use the undamaged, opposite strand as a template to ensure speedy and accurate DNA repair, something that can not be done for DSBs. Incorrect DNA repair causes genetic, and likely harmful, alterations of DNA molecules. In addition to being more easily self-repaired than DSBs, SSBs are also less biologically impactful and it follows that DSBs are the main cause of IR-induced cell death, despite the fact that the number of IR-induced SSBs by far exceeds the number of IR-induced DSBs following a typical radiotherapy dose. As an example, a radiation dose of 1 Gy yields approximately 1000 SSBs but only 40 DSBs [79].

\subsubsection{Influence of Oxygen on Radiotherapy Responses}

It is well established that in mammalian cells, IR-induced DNA damage is boosted by the presence of oxygen [82]. If the same radiation dose is delivered to two biological systems that are identical, save from the fact that one of the systems is hypoxic whilst the other system is well-oxygenated, the response (in terms of number of killed cells) will be greater in the well-oxygenated system. This can be explained by the fact that cellular oxygenation values affect the chemical process of IR-induced DNA radicals [83]. DNA radicals are formed when IR-induced free radicals interact with DNA molecules in a cell [83]. DNA radicals are typically short-lived but in the presence of oxygen, however, these short-lived DNA radicals are likely to bind to an oxygen molecule to form a stable DNA peroxide, thus rendering the DNA damage permanent (or fix). This is referred to as the oxygen fixation hypothesis [79]. If, on the other hand, molecular 


\section{MATHEMATICAL FRAMEWORK}

oxygen is sparse then it is unlikely that a DNA radical will bind to an oxygen molecule, and thus the DNA radical has a higher probability of chemically reducing back to its original (undamaged) DNA form. The oxygen fixation hypothesis is clarified in Example 2.6 .2

- Example 2.6.2: Consider a high energy electron $\left(e^{-}\right)$in the biological material resulting from $x$-ray exposure. If this electron strikes a water molecule $\left(\mathrm{H}_{2} \mathrm{O}\right)$, then this water molecule may split into a proton $\left(p^{+}\right)$and a hydroxyl radical (OH*) that can react with DNA (DNA-H) to form a DNA-radical (DNA*). If molecular oxygen $\left(\mathrm{O}_{2}\right)$ is available, then oxygen may bind to the DNA-radical to form a peroxy radical (DNA-OO*), which entails permanent DNA damage. On the other hand, if there is no molecular oxygen available, the DNA radical (DNA*) can be restored, i.e. repaired, to its original, undamaged form (DNA-H) 84].

It should also be noted that hypoxia may cause epigenetic and molecular cellular alterations that may modify radio-sensitivity in additional ways [85]. From the above discussion we have learnt that biological materials are the least radio-sensitive when completely oxygen deprived, and empirical results show that radio-sensitivity over oxygenation levels have a general sigmoidal trend [79]. To furthermore quantify the influence of oxygen on IR responses, a dose ratio, namely the oxygen enhancement ratio (OER) [82] is commonly used such that

$$
O E R_{m}=\frac{D^{N_{2}}}{D^{\text {air }}}
$$

where $D^{N_{2}}$ is some IR dose in nitrogen, and $D^{\text {air }}$ is the IR dose needed to achieve the same response in air. The subscript $m$ in $O E R_{m}$ here stands for maximum, as we are comparing radiotherapy responses in two extreme scenarios, specifically in nitrogen (with no oxygen) and in air (with plentiful oxygen). If the radiation response is evaluated in terms of a survival fraction of cells, or the number of DSBs, then $O E R_{m}$ takes the value of approximately $3 \pm 0.5$ for most eukaryotic cells. However, if the response is instead evaluated in terms of the number of SSBs, the OER value has a wider range of possible values [82], but since DSBs are the main cause of IR-induced cell death we here consider the value $3 \pm 0.5$ to be a good enough approximation to use in radiotherapy 
models in our mathematical framework. In order to describe the oxygen-dependence of radio-sensitivity, the oxygen enhancement ratio (OER) can be used where,

$$
\operatorname{OER}(\hat{K})=\frac{\mathrm{OER}_{m} \cdot \hat{K}+K_{m}}{\hat{K}+K_{m}}
$$

Here, $K_{m}=3 \mathrm{mmHg}$ is the oxygenation value achieving half of the maximum ratio and, as previously discussed, $\mathrm{OER}_{m}=3$ [67]. As is illustrated in Figure 2.5, Equation 2.15 yields the empirically observed sigmoidal curve.

\subsubsection{The Linear-Quadratic Model}

The linear-quadratic (LQ) model is ubiquitous in radiotherapy modelling. It describes the survival fraction, $S$, of a cell population as a function of a radiation dose, $D$, such that

$$
S=e^{-\left(\alpha D+\beta D^{2}\right)},
$$

where $\alpha$ and $\beta$ are cell-line specific radio-sensitivity parameters. The LQ model is used both in laboratories for analysing and predicting in vitro and in vivo experiments, and in clinical settings [79, 86]. If the surviving fraction $S$ in Equation 2.16 is plotted on a log-scale over the radiation dose $D$, then the response curve typically takes a quadratic (or a 'shouldered') shape. This curve is dominated by the $\alpha$-parameter for low $D$-values and by the $\beta$-parameter for high $D$-values. The curvature can be described by the ratio $\alpha / \beta$. For high $\alpha / \beta$-ratios, the curve is dominated by the linear term with coefficient $\alpha$ and thus assumes a fairly linear shape. On the other hand, for low ratios $\alpha / \beta$ the curve has a more quadratic shape. If radiation is given in multiple fractions, cells have time to repair sub-lethal damage in between doses, specifically, this repair usually takes 6-24 hours [86. If $n$ fractions with radiation dose $d$ are given, such that $n d=D$, then the LQ model can be adapted to express the survival as a result of multiple fractions where

$$
S=\left(e^{-\alpha d-\beta d^{2}}\right)^{n}=e^{-\left(\alpha n d+\beta n d^{2}\right)}=e^{-(\alpha D+\beta D \cdot d)} .
$$

Now, since $D \cdot d=D \cdot D / n<D^{2}$, the fractional LQ-model (Equation 2.17) will inherently result in a higher survival fraction $S$ than the single-dose LQ model (Equation 


\section{MATHEMATICAL FRAMEWORK}

2.16) given some dose $D$.

The current formalisation of the LQ model (Equation 2.16) does not have one single derivation, but is instead a result of contributions from multiple empirical and theoretical studies performed over several decades during the last century [86]. In the early 20th century, just a few years after the discovery of X-rays in 1895 [87, mathematical studies were performed that described in vitro radiation responses in simple organisms such as yeast, bacteria and viruses [86]. These studies reported exponential response curves when plotting survival fraction over radiation dose, and thus it was hypothesised that IR exposure causes a number of damaging events (or hits) proportional to the administered dose $D$. A cell was thought of as having one single sensitive target, and if such a target was hit by radiation, the cell would simply die [86]. If one assumes the hits to be Poisson distributed, with a mean value corresponding to the radiation dose $D$, then this 'Single Target - Single Hit' model yields an expression for cell survival as

$$
S=P_{h i t}(0, D)=e^{-\frac{D}{D_{0}}}
$$

where $P_{\text {hits }}(0, D)$ denotes the probability that there are exactly 0 hits on a target when a dose $D$ is administered. $D_{0}$ here denotes the dose causing on average one hit per cell.

- Remark 2.6.3: Recall that the Poisson distribution is a discrete probability distribution that describes the probability that a certain number of events occur in some fixed (here time) interval, when the events occur at a constant rate and independently of other events. Generally, the probability to observe $k$ events in some (time) interval is given by

$$
P(k \text { events })=e^{-\lambda} \frac{\lambda^{k}}{k !},
$$

where $\lambda$ is the expected number of occurrences. Hence, for the special zero-events case, $P(0)=e^{-\lambda}$.

Later on, in the 1950s, in vitro experiments with more complicated organism were carried out, now using cancer and non-cancer mammalian cell-lines. It was observed 
that mammalian cells are significantly more IR sensitive than are viruses and bacteria. Moreover, the empirically produced response curves were now shouldered, rather than exponential (like the ones previously observed in vitro for the simpler organisms) [86]. As a result, the 'Single Hit - Single Target' concept was modified in order to explain this shouldered response curve, and Both 'Single Hit - Multiple Targets' and 'Multiple Hits ÂĂ- Single Target' models were explored. In 'Single Hit - Multiple Targets' models, a cell is regarded as having multiple, specifically $m$, radio-sensitive targets that all need to be hit in order for the cell to die. If these targets are denoted $i$ where $i=1,2, \ldots, m$ then, with the average number of hits per target being $D / D_{0}$, the probability that a target $i$ gets hit is given by

$$
1-P_{h i t i}(0, D)=1-e^{-\frac{D}{D_{0}}}
$$

hence the probability that all targets $1,2, \ldots, m$ in a cell are hit is given by

$$
\left(1-P_{\text {hit } 1}(0, D)\right)\left(1-P_{\text {hit } 2}(0, D)\right) \ldots\left(1-P_{\text {hit } m}(0, D)\right)=\left(1-e^{-\frac{D}{D_{0}}}\right)^{m},
$$

and thus the probability that the cell survives, i.e. fewer than $m$ of its targets are hit, is

$$
S=1-\left(1-e^{-\frac{D}{D_{0}}}\right)^{m}
$$

Equation 2.22 yields a shouldered response curve for 'Multiple Hit - Single Target' models. Similar result-trends were obtained for 'Single Target - Multiple Hits' models for radiation dosages within the range typically studied, but for dosages outside the typical range these models produced response curves that did not agree with experimental data [86]. This prompted the exploration of 'Multiple Target - Multiple Hits' models but theses required more parameters and were thus more difficult to fit to experimental data. In the meanwhile, studies using the 'Single Hit - Multiple Targets' model reported a wide array of $m$-values, i.e. number of targets. For example, $m=2$ was reported for HeLa cells but in other studies the $m$-value was found to be up to two orders of magnitude larger [86]! Consequently, $m$ was, and is still, instead considered to be an empirical factor describing the specific system at hand, and models other than 'Hit(s) - Target(s)' 


\section{MATHEMATICAL FRAMEWORK}

models were developed using alternatives to this Target theory. This resulted in several $L Q$-like models being suggested with various motivations. In 1966, Sinclair wrote down the LQ-model (Equation 2.16 based on empirical observations, and in the 70s, theoretical motivations for the linear-quadratic response-curve were published. It was suggested that the linear part corresponds to single track events whilst the quadratic part corresponds to multiple track events. In abstract models, a cell was thought of as either dying directly from IR-induced DNA damage or by progressing through sublethal stages in order to eventually die, and in mechanistic models, DSBs were said to be inflicted by damage resulting from either one single particle or multiple particles [86]. When simplified, the abstract and mechanistic model both suggest linear-quadratic response curves [86].

Besides being verifiable in laboratories and theoretically supported, the LQ model has proven applicable in clinical settings [86], making it the first choice radiotherapymodel for many mathematical oncologists. In the mathematical framework used in this thesis, we are working with agent-based models in which each agent has an individual response to IR. Thus the LQ-model (Equation 2.16) is here modified to describe the probability that one agent (e.g. one individual cell) survives a radiation dose $D$. Cellular responses to radiotherapy are dependent on oxygenation status [88], cell cycle progression [89, 90], and cell-line characteristics [2]. To honour this, the survival probability of an agent in lattice point $x$ at time $t$ is here given by

$$
S(x, t)=e^{-D\left([O M F] \alpha(x, t)+D[O M F]^{2} \beta(x, t)\right)},
$$

where $D$ is the radiation dose, $n$ is the number of administered radiation fractions and $\alpha$ and $\beta$ are cell-line specific sensitivity parameters [23]. To include cell cycle sensitivity, $\alpha$ and $\beta$ are here cell cycle dependent [2], and the oxygen modification factor (OMF) is incorporated to include oxygen sensitivity [91]. The OMF function is a scaled version of the OER function (Equation 2.15), suitable for stochastic models, here re-written into a form that is appropriate for agent-based models such that

$$
\operatorname{OMF}(\hat{K}(x, t))=\frac{\operatorname{OER}(\hat{K}(x, t))}{\operatorname{OER}_{m}}=\frac{1}{\operatorname{OER}_{m}} \frac{\operatorname{OER}_{m} \cdot \hat{K}(x, t)+K_{m}}{\hat{K}(x, t)+K_{m}},
$$




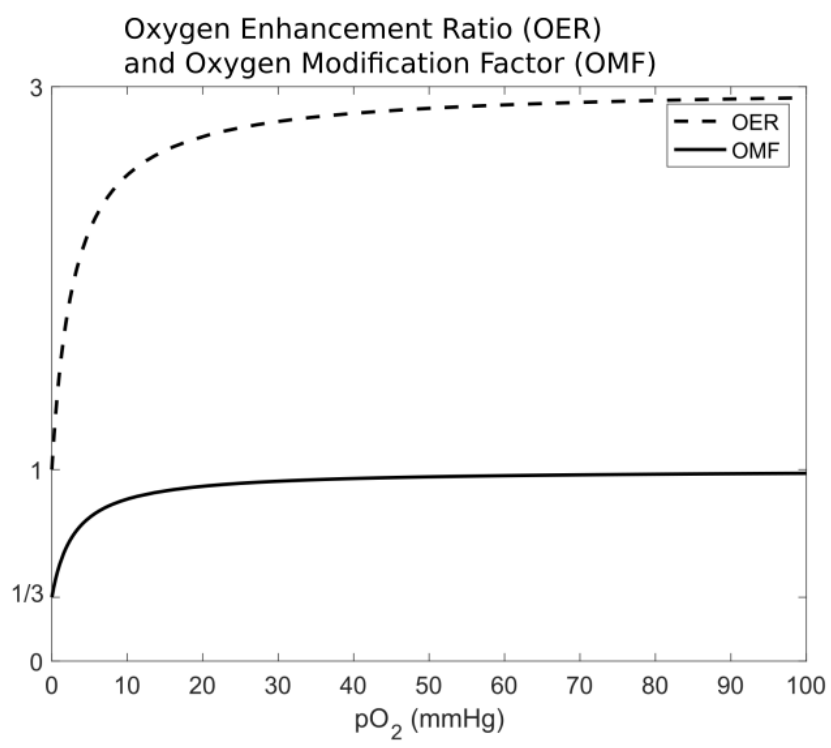

Figure 2.5: The oxygen enhancement ratio (OER) and the oxygen modification factor (OMF) plotted over oxygenation value.

where $\hat{K}_{x, t}$ denotes the oxygenation value (in units of $\mathrm{mmHg}$ ) in lattice point $x$ at time $t$, and the values for $K_{m}$ and $O E R_{m}$ are previously defined under Equation 2.15 The OER and OMF functions are illustrated in Figure 2.5. The survival probability of a cell exposed to a typical clinical radiotherapy dose 2 Gy is plotted in Figure 2.6, as a function of oxygenation and cell cycle phase, where the sensitivity parameters $\alpha$ and $\beta$ are chosen to correspond to those of typical cancer cell lines [2]. 


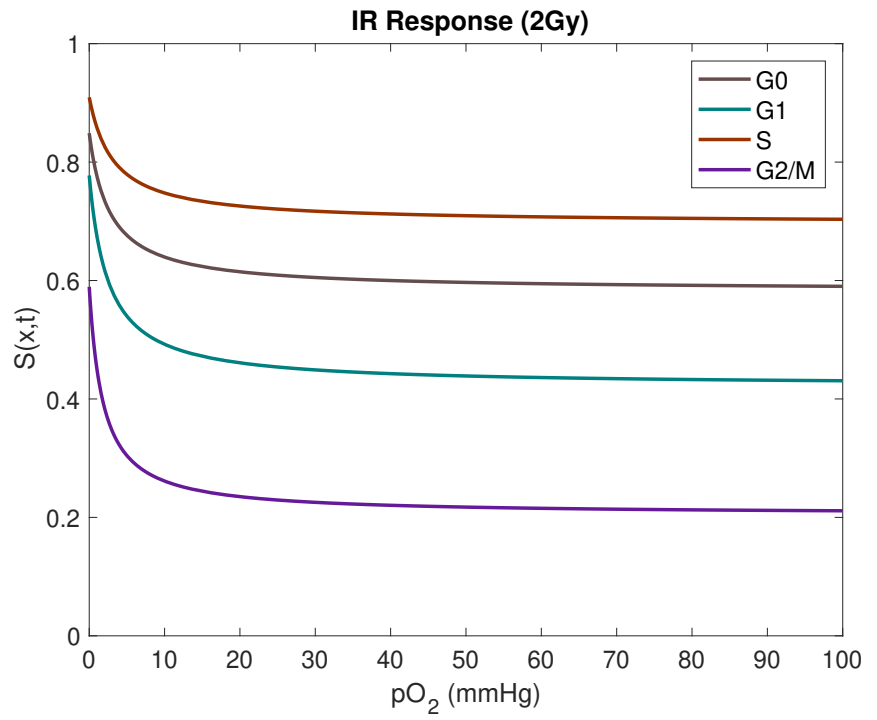

Figure 2.6: The probability $S(x, t)$ that a cell location $x$ at time $t$ in our mathematical framework survives a radiation dose of $2 \mathrm{~Gy}$, as a function of its cell cycle phase (see legend) and oxygenation value. The values for $\alpha$ and $\beta$ are gathered from a previous study by Kempf et al. [2] and are listed in Table 4.1 . 


\section{Chapter 3}

\section{Drug Resistance}

Cancer cells can be, or become, resistant to chemotherapeutic drugs. In this chapter we investigate what happens when cancer cell populations, comprising both susceptible and resistant cancer cells, are subjected to various chemotherapy treatments plans.

\subsection{Chapter Summary}

Tumour recurrence post chemotherapy is an established clinical problem and many cancer types are often observed to be increasingly drug resistant subsequent to chemotherapy treatments. Drug resistance in cancer is a multipart phenomenon which can be derived from several origins and in many cases it has been observed that cancer cells have the ability to possess, acquire and communicate drug resistant traits.

In this chapter, the in silico framework described in Chapter 2 is adapted in order to study drug resistance and drug response in cancer cell populations exhibiting various drug resistant features. The framework is based on an on-lattice hybrid multiscale mathematical model and is equipped to simulate multiple mechanisms on different scales that contribute towards chemotherapeutic drug resistance in cancer. This study demonstrates how drug resistant tumour features may depend on the interplay amongst intracellular, extracellular and intercellular factors. On a cellular level, drug resistant cell phenotypes are here derived from inheritance or mutations that are spontaneous, drug-induced or communicated via exosomes. Furthermore intratumoural heterogeneity 


\section{DRUG RESISTANCE}

and spatio-temporal drug dynamics heavily influences drug delivery and the development of drug resistant cancer cell subpopulations. Chemotherapy treatment strategies are here optimised for various in silico tumour scenarios and treatment objectives. We demonstrate that optimal chemotherapy treatment strategies drastically depend on which drug resistant mechanisms are activated, and that furthermore suboptimal chemotherapy administration may promote drug resistance.

\subsection{Introduction}

Chemotherapy is one of the major anticancer therapies, it is widely used both by itself and as part of multimodality treatment strategies. In most cases chemotherapy is effective, however the existence, or the development, of chemotherapeutic drug resistance in tumours continues to be a major problem in chemotherapeutic treatments, often leading to tumour recurrence post treatment [92 $[98]$. Clinical and experimental observations suggest that cancers are often increasingly drug resistant subsequent to chemotherapy exposure [98-101] and moreover cancer cells have the ability to posses, acquire and communicate drug resistant traits, enabling them to survive in the presence of chemotherapeutic drugs [95]. The existence of drug resistant phenotypes in cancer cell populations significantly impacts the efficacy and successfulness of chemotherapy [102-104].

The emergence of drug resistant cancer cells in tumours results in multiple subpopulations comprising drug sensitive (S) and drug resistant (DR) cells [102]. Furthermore cancer cell populations may evolve according to Darwinian principles [105] and cells that acquire drug resistance during chemotherapy have been observed to be increasingly metastatic [99], consequently DR subpopulations can reach significant proportions despite initially accounting only for a small fraction of some cancer cell population [95]. S and DR subpopulations that coexist synergistically compete for resources such as space and nutrients [105, 106], this competition influences the tumour environment and yields intratumoural heterogeneity. In clinical cases where tumour eradication is implausible, chronic control treatments can be proposed in which tumours are continuously managed and prohibited from reaching lethal proportions [95, 106, 107], long-term chemotherapy treatments are however linked to high frequency drug resistance [108, 109]. Since 
DR subpopulations are more fit than $\mathrm{S}$ subpopulations to survive in the presence of drugs, repeated or prolonged chemotherapy administration may amplify this fitness differentiation. Indeed Duan et al. [102] performed in vitro and in vivo experiments to conclude that in absence of drugs, sensitive cells are more fit than drug resistant cells and conversely, in presence of drugs DR subpopulations dominate as $\mathrm{S}$ subpopulations are reduced [102]. Thus drug resistant cells may thrive in micro-environments containing chemotherapeutic drugs, and a large DR subpopulation may result in disease recurrence post chemotherapy [110]. Ensuring that the DR subpopulation does not dominate the $\mathrm{S}$ subpopulation is of importance as such an outcome would render the tumour uncontrollable by chemotherapy [106]. This suggests that deliberately maintaining a subpopulation of drug sensitive cells may constitute a strategic countermeasure in tumour control schemes [111]. Duan et al. investigated the plausibility of this proposed strategy in vivo by comparing two cell populations exposed to chemotherapeutic drugs [102]. The first of these cell populations comprised drug resistant cells only, and the second population contained a combination of both drug resistant and sensitive cells. Their study confirmed that the second, combined, cell population was controllable by chemotherapy for a longer time period than the first, drug resistant, cell population [102].

Drug resistance is a multipart phenomenon which can be derived from several origins, in fact a cancer cell or tumour may express drug resistance in various ways [95, 111, 112]. Drug resistance may arise due to micro-environmental or intrinsic cell factors [113] and cells can acquire drug resistance by for example amplifying drug target molecules, activating DNA-repair, inducing drug transporters or altering their drug metabolism [112]. Phenotypical variations in cells, such as drug resistance, can be inherited or acquired and further, cells may be resistant to one specific drug or to multiple drugs, the latter phenomenon is known as multidrug resistance (MDR) [95, 105, 108, 114]. Early work performed by Luria and Delbrück on bacteria indicated that virus resistant mutations occur independently of the virus itself, thus indicating the existence of primary virus resistance [115]. These findings have since been adapted to oncology [93, 95], and primary drug resistance, that is drug resistance that occurs independently of the drug presence, is an accepted phenomenon arising from cell mutations. However, drug presence has 


\section{DRUG RESISTANCE}

been demonstrated to speed up the development of DR subpopulations [116] and cancer cells may acquire drug resistance by altering their genetic or epigenetic structure in order to evade drug effects [93. Such alterations are induced by drug presence and may include dislodging drug receptors or overexpressing and modifying target molecules [93]. Heat shock proteins (Hsps) are molecular chaperones, continuously present in eukaryotic cells, yielding cytoprotective cell effects [99, 117]. Via their chaperoning actions they enable cells, both healthy and cancerous, to adapt to extracellular variations and maintain homeostasis whilst subjected to external stresses such as, maybe most importantly, hyperthermia but also hypoxia and anoxia, toxins and the presence of harmful chemical agents such as chemotherapeutic drugs [99, 101, 117, 118]. In healthy cells, the upregulation of Hsps can protect cells from for example high temperatures [99], however in cancerous cells Hsp upregulation may protect cells from drug effects [99, 101], thus enabling cells to survive under otherwise lethal conditions [117]. By extension Hsps have been linked to resistance to chemotherapeutic drugs [99] such as cisplatin, doxorubicin 101] and bortezomib [118.

Typical chemotherapy drugs target cells in active cell cycle phases, thus quiescent cells parry drug effects [96] and similarly slow-cycling cells are intrinsically more drug resistant than fast cycling cells [110, 119] as they are more likely to evade drug attacks. Slow-cycling cells have been linked to cancer stem cell-like (CSC-like) cells [96], they are important drivers for tumours due to their increased drug-survival rate and ability to serve as reserve stem cells [110, 119]. CSC-like cells have been depicted to display various traits including being slow-cycling, migratory and non-adhesive [92]. Rizzo et al. [100] demonstrated in vivo in mouse tails that a subpopulation of CSC-like cells indeed may benefit from drug presence when competing for resources with other cell populations. Thus slow-cycling cells have been identified to reinforce tumours, hence to eradicate cancer cell populations containing a subpopulation of slow-cycling cells it is crucial to target both slow-cycling and fast-cycling cells [119]. Intercellular communication is vital for multicellular organisms and cells may communicate with each other using chemical signalling, direct physical contact or, as discussed here, sending and receiving exosomes [109, 120]. Out of these listed information mediators, exosomes are of particular interest as they are detectable, cell type-specific and able to travel long distances [109, 120]. This implies that they could potentially constitute therapeutic 
targets or biomarkers and thus be used to impede or signal cancer [109, 120]. Exosomes constitute subcellular 'molecule parcels' that cells may utilise to dispose of non-essential materials [120], however perhaps more interestingly, they also facilitate long-distance intercellular communication by transporting information from sender cells to recipient cells [109, 121, 122]. These molecule-parcels contain biomolecules such as proteins, mRNA and DNA which may provide recipient cells with information that can be used to alter phenotypical attributes, in order to increase fitness [120]. Exosomes are a type of extracellular vesicle (EV) [123] and recent studies have identified EVs as key players in cancer development as they can influence tumour growth and metastasis by communicating oncogenic information [120]. EVs have also been assumed to be a part of the process that converts non-malicious cells into cancerous, and of optimising the balance between CSCs and non-CSCs [120]. Thus in response to chemotherapeutic drugs, cancer cells may not only develop individual drug resistance, but furthermore they may render other cells drug resistant by secreting exosomes to communicate and share drug resistant traits [123]. Exosomes may induce both destructive and protective cell responses, in fact the role of EVs depends on the regarded scenario [124]. In this study pathogenic exosomes only are modelled.

Mathematical models of tumour growth and treatment response may further cancer research by contributing insight into tumour dynamics, elucidating and validating clinically and experimentally recognised phenomena and guiding in vitro and in vivo experiments [95, 103, 105-107, 125]. Computational approaches to simulate biological systems are an important part of theoretical biology and may provide insights into biological phenomena [126]. In silico experiments have the advantage of cheaply being able to reproduce biological systems that span long time periods faster than real-time [105] and they can be used to find optimal treatment scheduling [11, 127]. Various such mathematical models of tumour growth, treatment response and drug resistance have previously been proposed [95, 128, 134]. Roose et al. presented a comprehensive review of models of avascular tumour growth [133] and Lavi et al. compiled an extensive report discussing previous work on mathematical models of drug resistance in cancer [95]. To name a few such models, Monro et al. [106] presented a continuum model in which tumour growth follows Gompertzian dynamics and drug resistant mutations occur proportionately to the tumour growth rate, in accordance with Luria Delbrück 


\section{DRUG RESISTANCE}

models. They concluded that increased drug administration may in fact reduce the survival length of a patient. Powathil et al. [132] used the Compucell3D framework [135] to investigate two coexisting subpopulations, specifically one fast-cycling and one slowcycling, in the presence of drugs to demonstrate intrinsic drug resistance of slow-cycling cells. There currently exists a number of hybrid discrete-continuum mathematical models that account for the multiscale nature of cancer [50], these models can be used to study tumour behaviour in response to multimodality treatment schemes [136-142]. Several modelling attempts have been made to address the multiscale aspects of cell growth by incorporating details such as vascular dynamics, oxygen transport, hypoxia, cell division and other intracellular features in order to study tumour dynamics and treatment response [19, 57, 143, 144]. Recently, Powathil et al. [6, 145] developed a hybrid multiscale cellular automaton, integrating cell cycle dynamics and oxygen distribution to study cell cycle-based chemotherapy delivery in combination with radiation therapy. As an important step towards personalised medicine, Caraguel et al. [146] managed to create virtual clones of in vivo tumours in mice using multiscale hybrid modelling. The tumour growth of various mouse tumours successfully agreed with the tumour growth of their respective virtual clones. Details of other multiscale cancer models are available in a review by Deisboeck et al. [56]. The mathematical models that are referenced in this section are categorised and summarised in Table 3.1 (where models from review papers have been omitted for brevity).

In the present in silico study, we propose a hybrid multiscale mathematical model that incorporates multiple types of drug resistance. In silico experiments are performed in order to study chemotherapeutic drug response in heterogeneous cancer cell populations hosting various types of drug resistant phenotypes pre, peri and post chemotherapy.

\subsection{Model and in silico Framework}

In this study, we expand on the mathematical framework presented in Chapter 2 to incorporate multiple types of mechanisms that elicit drug resistance in cancer cells. Specifically, the CA used in this study can be categorised as a hybrid multiscale on-lattice model [50], incorporating a non-uniform micro-environment, extracellular dynamics, intracellular dynamics, intercellular dynamics and various categories of drug resistance 


\begin{tabular}{|c|c|c|c|c|c|c|c|}
\hline model reference & 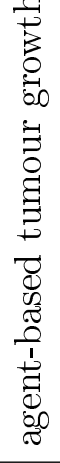 & 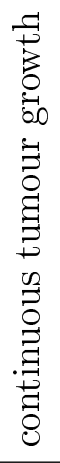 & 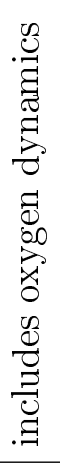 & 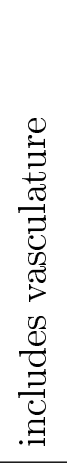 & 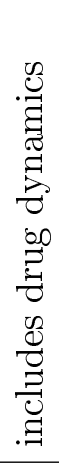 & 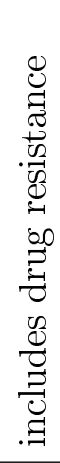 & 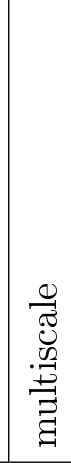 \\
\hline Panetta et al. [130] (1996) & & $\checkmark$ & & & $\checkmark$ & $\checkmark$ & $\checkmark$ \\
\hline Macklin et al. [19] (2009) & $\checkmark$ & & $\checkmark$ & & & & $\checkmark$ \\
\hline Monro et al. [106] (2009) & & $\checkmark$ & & & $\checkmark$ & $\checkmark$ & $\checkmark$ \\
\hline Owen et al. [143] (2009) & & $\checkmark$ & $\checkmark$ & $\checkmark$ & & & $\checkmark$ \\
\hline Perfahl et al. [144] (2011) & $\checkmark$ & & $\checkmark$ & $\checkmark$ & $\checkmark$ & & $\checkmark$ \\
\hline Caraguel et al. [146] (2016) & $\checkmark$ & & $\checkmark$ & $\checkmark$ & & & $\checkmark$ \\
\hline Perez-Velazquez et al. [131] (2016) & $\checkmark$ & & $\checkmark$ & $\checkmark$ & $\checkmark$ & $\checkmark$ & $\checkmark$ \\
\hline Powathil et al. [147] (2016) & $\checkmark$ & & $\checkmark$ & $\checkmark$ & $\checkmark$ & $\checkmark$ & $\checkmark$ \\
\hline Lorz et al. [129] (2017) & & $\checkmark$ & & & $\checkmark$ & $\checkmark$ & $\checkmark$ \\
\hline
\end{tabular}

Table 3.1: A table of mathematical models (referenced in Chapter 3.2) that include various aspects of cancer growth. A check mark $(\checkmark)$ indicates that the referenced model uses/includes/is what is specified in the respective columns.

regarded on a cellular resolution. The CA model uses partial differential equations (PDEs), ordinary differential equations (ODEs) extracted from a regulatory molecular network, as well as stochasticity and phenomenological rules formulated by observations from biological experiments and clinical reports. An overview of the model schematics are illustrated in Figure 3.1 and details are provided throughout this section. The CA here extends in two spatial dimensions, specifically a 100 by 100 square grid is utilised to simulate a physical tissue slab of $(2 \mathrm{~mm})^{2}$. This agrees with biological dimensions and each grid point is either occupied by a cancer cell or a blood vessel cross section, or it is empty (i.e. occupied by extracellular matrix only) [6]. At the start of the in silico experiment, one initial cancer cell is planted at the centre of the grid, over time this cell divides to give rise to a population of cancer cells and eventually chemotherapeutic drugs are applied to the system. Blood vessels are non-equidistantly scattered across the grid, they are assumed stationary and perpendicular to the two-dimensional tissue slab. Thus blood vessel cross sections live on the grid, where they act as source points for oxygen and drugs. 


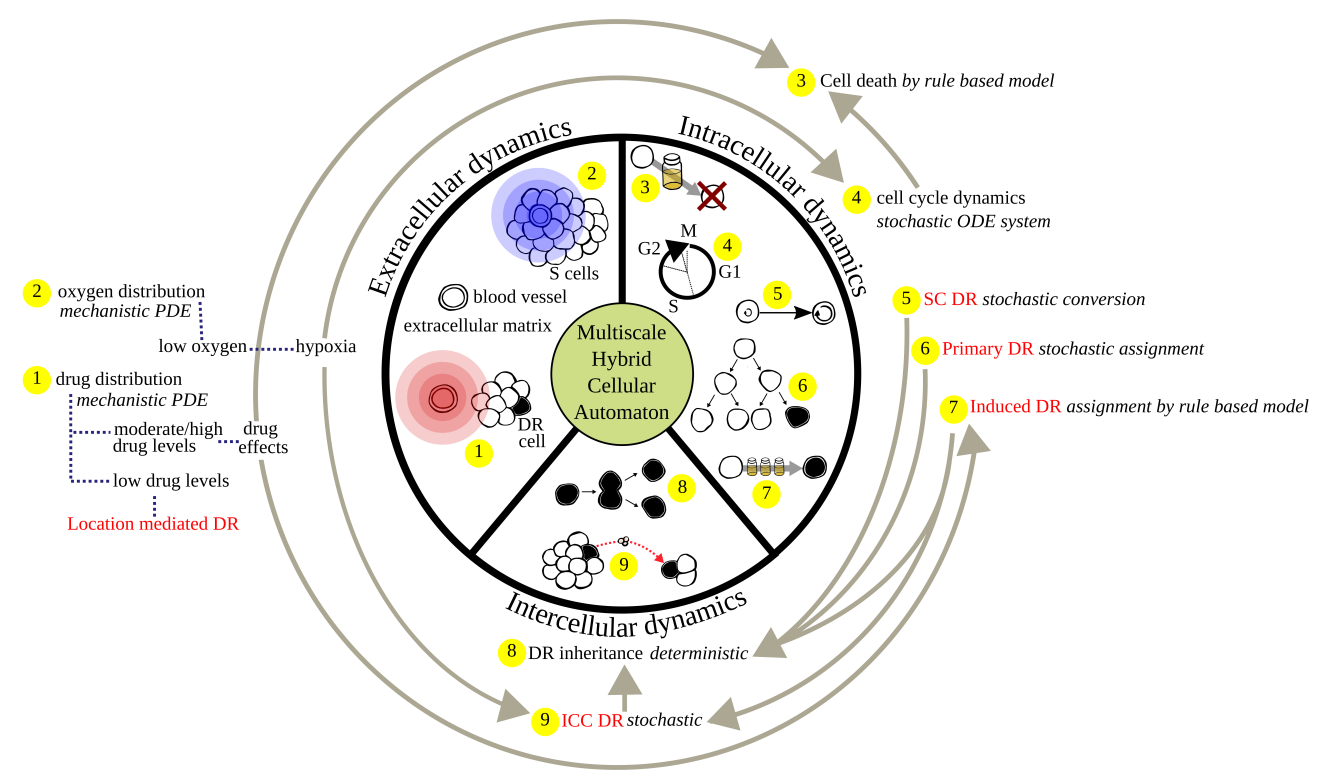

\section{Model image key}

1 Drug distribution Model: Mechanistic diffusion-reaction equation. source points are blood vessels. Effect: Moderate to high drug levels prompt responses in cells whilst cells in low drug regions evade drug effects implicitly by Location mediated DR.

2 Oxygen distribution Model: Mechanistic diffusion-reaction equation. source points are blood vessels. Effect: Low oxygen levels yield hypoxic regions.

3 Cell death Model: Rule based. A cell dies if the cell drug level sufficiently high (effect from 1) and the cell is in the cell cycle phase targeted by the drug (effect from 4)

4 Cell cycle dynamics Model: Regulated by a molecular network described by a system of ODEs with stochastic parameters. Hypoxia delays cell cycle progression (effect from ${ }^{2}$ ).
5 SC DR Model: Stochastic conversion. By converting into a slow-cycling state cells may implicitly evade drug effects, and thus become implicitly drug resistant phenotypes.

6 Primary DR Model: Stochastic assignment. A daughter cell may be 'born' as a drug immune phenotype despite having a drug sensitive mother cell.

7 Induced DR Model: Rule based. A cell exposed to high, but non-lethal, drug levels for a certain period of time aqcuires drug resistance (effect from 1 ).

8 DR inheritance Model: Deterministic. Drug resistant phenotypes (5/6/7/9) pass on drug resistance to daughter cells.

9 ICC DR Model: Stochastic exosome activity. Cells that have aqcuired drug resistance via Induced DR ( 7 ) or ICC DR ( 9 ) may communicate via exosomes to pass on drug resistant traits to sensitive cancer cells. Exosome activity is higher in hypoxic regions (effect from ${ }^{2}$ ).

Figure 3.1: A schematic representation of the multiscale hybrid mathematical model used in this study. The model integrates extracellular, intracellular and intercellular dynamics. This is an on-lattice model and a lattice point may be occupied by a sensitive $(\mathrm{S})$ or drug resistant (DR) cancer cell shown in black, a blood vessel cross-section or extracellular space. Various categories of drug resistance regarded on a cellular resolution are incorporated in the model, these categories are marked in red and listed in Table 3.4. 


\subsubsection{Intratumoural Heterogeneity}

Tumours are dynamic and should therefore be modelled as such [112], they are also heterogeneous and may constitute multiple distinguishable subpopulations [105, 112]. Intratumoural heterogeneity has been observed to promote drug resistance [103, 112 ] and hinder successful tumour prediction, thus by extension intratumoural heterogeneity complicates intelligent chemotherapy administration [105]. A tumour can express intratumoural heterogeneity in various ways and on multiple scales. For example nutrient concentrations, cell cycle dynamics and drug resistant traits may vary amongst cells in a tumour [103, 105]. Phenotypical attributes, such as drug resistant traits, may be acquired or inherited [105] and moreover stochasticity occurs naturally in biological processes. Hence various phenotypical subpopulations may arise in a cancer cell population, even if the population originates from one single cell [112]. To effectively treat tumours one should thus account for intratumoural heterogeneity, including the potential uprising of drug resistant subpopulations [105]. Our model accounts for intratumoural heterogeneity on various scales, details are provided in the following subsections. On a cellular level, each cell has an individual cell cycle length and individual drug resistant traits. On an extracellular level, the spatio-temporal micro-envrironment is highly dynamic, each cell has its own neighbours and moreover the blood vessels are non-equidistantly placed that oxygen and drug concentrations vary asymmetrically across the grid.

\subsubsection{Intracellular Dynamics}

The cell cycle mechanism is here partitioned into four sequential main phases, namely the gap 1 (G1), synthesis (Syn), gap 2 (G2) and mitosis (M) phase. (Note that we, in this chapter, use the abbreviation 'Syn' do denote the synthesis phase as the abbreviation 'S' is reserved to denote drug sensitive cells). We here use the regulatory molecular network, described by the ODE system (Equation 2.1 in Chapter 2) to model the cell cycle progression of each individual cell. Cancer cells are thus here categorised as being in either the G1 phase or in the collective Syn-G2-M phase of the cell cycle, or alternatively cells can exit the cell cycle and enter the quiescent phase G0 [6]. When cell division occurs at time step $t_{c d}$ in the model, a daughter cell is placed on a grid point in the spherical neighbourhood of the parental cell, located in point $x_{\text {parent }}$. At cell division the 


\section{DRUG RESISTANCE}

mass of the parent cell is halved so that $[$ mass $]\left(x_{\text {parent }}, t_{c d}\right)=\frac{1}{2} \cdot[$ mass $]\left(x_{\text {parent }}, t_{c d}-1\right)$ [60]. Grid points in lower order neighbourhoods are prioritised in this process, however up to third-level neighbourhoods are regarded. Each cell may divide until there is no unoccupied grid point on which to place a daughter cell, when this occurs a cell enters the quiescent phase G0. A cell may however re-enter the cell cycle if its neighbourhood is freed up and space is made available. Whilst in a quiescent phase cells are assumed to be drug immune in our model, this is because classical chemotherapy drugs target molecules that are over-expressed in specific cell cycle phases, for example the drug cisplatin affects the G1 phase of the cell cycle [6, 148].

\subsubsection{Extracellular Dynamics}

As is previously described in Chapter 2.4, extracellular dynamics is modelled using PDEs describing oxygen and drug distribution. Oxygen is continuously produced at each time step on the blood vessels cross sections from which it is distributed across the grid according to the following diffusion-reaction equation [6],

$$
\frac{\partial K(x, t)}{\partial t}=\nabla \cdot\left(D_{k}(x, t) \nabla K(x, t)\right)+r_{k}(x, t) m(x)-\phi_{k} K(x, t) \operatorname{cell}(x, t),
$$

where $K(x, t)$ denotes oxygen concentration in location $x$ at time $t, D_{k}(x, t)$ is the oxygen diffusion coefficient, $r_{k}(x, t)$ is the oxygen production rate and $\phi_{k}$ is the oxygen consumption rate. The variables $m(x)$ and $\operatorname{cell}(x, t)$ are binary so that $m(x)=1$ if there is a blood vessel in location $x$ and $m(x)=0$ otherwise. Likewise $\operatorname{cell}(x, t)=1$ if there is a cell in location $x$ at time $t$ and $\operatorname{cell}(x, t)=0$ otherwise. No-flux boundary conditions are applied, such boundary conditions coupled with the oxygen production at each time step will cause the total oxygen in the system to fluctuate over time. Thus in accordance with previous work by Powathil et al. [67], the absolute hypoxic threshold value will be different at each time step in the simulation, whilst the relative, or scaled, hypoxic threshold value will remain the same over time. This approach yields a spatial oxygen distribution at each time step which can be used to evaluate hypoxia. Physically, a cell is here classified as being hypoxic if its partial pressure of oxygen $\left(\mathrm{pO}_{2}\right)$ is $10 \mathrm{~mm} \mathrm{Hg}$ or less [149]. Following Powathil et al. [6], a grid point in the implementation is defined to be hypoxic if it has a relative oxygen concentration of 
less than 0.1 , where an oxygen concentration of 1 is normalised at the grid point with the highest oxygen concentration on the grid. Oxygen diffuses slower over grid points occupied by cancer cells than elsewhere and sufficiently high oxygen concentrations promote rapid cell proliferation, whilst hypoxia hinders cell cycle advancement. These hypoxic effects are incorporated in the model via the $[H I F]$ parameter occurring in Equation 2.1 [6]. Chemotherapy drugs are similarly administered via blood vessel cross sections, however drugs are instantaneously produced at one single time step per drug administration. Drugs diffuse according to

$$
\frac{\partial \mathscr{C}(x, t)}{\partial t}=\nabla \cdot\left(D_{c}(x, t) \nabla \mathscr{C}(x, t)\right)+r_{c}(x, t) m(x)-\phi_{c} \mathscr{C}(x, t) \operatorname{cell}(x, t)-\eta_{c} \mathscr{C}(x, t),
$$

using no-flux boundary conditions. Here $\mathscr{C}(x, t)$ denotes drug concentration in location $x$ at time $t, D_{c}(x, t)$ is the drug diffusion coefficient, $r_{c}(x, t)$ is the drug production rate, $\phi_{c}$ is the drug consumption rate and $\eta_{c}$ is the drug decay rate. Chemotherapy drugs diffuse faster across extracellular space than inside the tumour [6]. Provided that the cell is in the drug-targeted cell cycle phase and that the cell is not explicitly drug resistant, a cell in location $x$ at time $t$ is killed if the drug concentration $\mathscr{C}(x, t)$ is such that $\mathscr{C}(x, t) \geq \kappa$, where $\kappa$ is the lethal threshold drug concentration. When a cell dies, its grid point $x$ becomes empty. Parameters occurring in the PDEs are listed in Section 3.3 .3 .1

\subsubsection{Parameters for Oxygen and Drug Distribution}

\begin{tabular}{llll}
\hline Parameter & Symbol & Value & Reference \\
\hline Oxygen diffusion coefficient & $D_{k}$ & $2.5 \cdot 10^{-5}\left[\mathrm{~cm}^{2} s^{-1}\right]$ & {$[150]$} \\
Oxygen supply rate & $r_{k}$ & $8.2 \cdot 10^{-3}\left[\mathrm{~s}^{-1}\right]$ & {$[151]$} \\
Oxygen consumption rate & $\phi_{k}$ & $2 \cdot 10^{-1}\left[\mathrm{~s}^{-1}\right]$ & computed $^{(1)}$ \\
Cisplatin diffusion coefficient & $D_{c}$ & $7.6 \cdot 10^{-6}\left[\mathrm{~cm}^{2} s^{-1}\right]$ & computed $^{(2)}$ \\
Cisplatin consumption rate & $\phi_{c}$ & $0($ negligible $)$ & {$[6]$} \\
Cisplatin decay rate & $\eta_{c}$ & $1.316\left[\mathrm{~h}^{-1}\right]$ & estimated from [152] \\
\hline
\end{tabular}

Table 3.2: Parameters for Equations 3.1 and 3.2. (1) $\phi_{k}$ is computed using the relationship $L=\sqrt{D / \phi}$ [6]. (2) $D_{c}$ is computed using the relationship $D_{c} / D_{k}=$ $\sqrt{\text { molecular weight of } \mathrm{O}_{2}} / \sqrt{\text { molecular weight of drug }}[7$, where molecular weight are gathered from the PubChem data base [8]. 


\section{DRUG RESISTANCE}

Diffusion and production rates of oxygen and drugs vary across the grid so that [6] $D_{i}(x, t)= \begin{cases}\frac{D_{i}}{1.5} & \text { in the tumour } \\ D_{i} & \text { elsewhere }\end{cases}$

for $i=k, c$ and

$r_{k}(x, t)=\left\{\begin{array}{l}\frac{r_{k}}{1.5} \text { in the tumour, } \quad r_{c}(x, t)=\left\{\begin{array}{l}\frac{r_{c}}{1.5} \text { in the tumour } \\ r_{k} \text { elsewhere, }\end{array} \text { on the tumour boundary }\right. \\ \frac{r_{c}}{2} \text { outside tumour. }\end{array}\right.$

The drug decay rate $\eta_{c}$ is estimated to match the half-life time of cisplatin which has been reported as $31.6 \pm 6$ minutes [152]. Cisplatin supply is here modelled as instantaneous and thus equal to $r_{c}$ at the two time steps conferring with drug administration ( $\mathrm{t}=500$ hours and $\mathrm{t}=600$ hours) and zero for all other time steps. Here, $r_{c}$ is estimated and scaled according to the chosen drug dosage so that

$$
N_{i} \cdot \mathrm{C}=r_{c} \cdot(\text { number of blood vessels }),
$$

hence $r_{c}$ corresponds to the amount of drug produced at one blood vessel cross section at one time step. Here $i=0,1,2,3,4$ and $N_{i}=0,1,2,4,8$ in accordance with the possible drug dosages explored in the in silico experiment which are $1 \mathrm{C}, 2 \mathrm{C}, 4 \mathrm{C}$ and $8 \mathrm{C}$. $\mathrm{C}$ and $\kappa$ are parameterised so that $1 \mathrm{C}$ kills half of the cell population in absence of any included drug resistant mechanisms, more specifically, here $\mathrm{C}=10^{4}$ (equal to the number of grid points) and $\kappa=0.18$. Since $\mathrm{C}$ is equal to the number of grid points, all cancer cells would die immediately from drug exposure if drugs were produced homogeneously across the grid, as such a scenario would yield a drug concentration $\mathscr{C}(x, t)=1>\kappa \forall x$.

\subsubsection{Drug Resistance}

In this study, drug resistance is regarded on a cellular resolution, thus subcellular mechanics are simplified and drug resistance is categorised into cellular-level categories as illustrated in Figure 3.2 . In the model drug resistance is firstly categorised as being either explicit or implicit. Explicit drug resistance occurs when a cell possesses any subcellular trait that directly protects it from drug effects, rendering it immune to some drug. Conversely, a cell displays implicit drug resistance when it is shielded from drug impact due to some indirect reason such as being slow-cycling or spatially located in a region of low drug concentration (location mediated DR). Explicit drug resistant 
traits can be induced or inherited [153, 154] and also, cells may perform phenotypical alterations in response to intercellular interactions [112]. Therefore we here categorise drug resistance as being induced, primary or communicated. Induced drug resistance is activated in cells as a defensive response to drug presence whilst primary drug resistance is caused by cell mutations occurring independently of drugs [153, 154]. Cancer cells in which drug resistance has been induced may communicate and spread their drug resistant traits to other cancer cells via intercellular communication (ICC) in an effort to secure species survival [109, 120, 123]. Using hybrid modelling, the various categories of drug resistance are here modelled in different ways in order to easily reproduce biological phenomena in a way that is consistent with available clinical and experimental observations and data. In our model, drug resistance obeys rules formulated from previous findings from in vitro and in vivo results. Also incorporated are stochastic methods, as stochasticity occurs naturally in biological processes and may generate different cell phenotypes [112]. For all categories of phenotypical drug resistance, it is assumed that once a cell has established a drug resistant trait, its offspring will inherit that trait. This is in accordance with evolutionary Darwinian principles, as DR subpopulations are more fit to survive in drug presence than are S subpopulations [155]. Furthermore, the micro-environment influences drug transport across the tumour and impeded drug delivery by poor diffusion is indeed one of the primary reasons for treatment failure [95]. Thus drug efficacy and cytotoxic cell death is affected by the micro-environment, since drugs may not reach target cells. This may occur if the drug diffusion is impeded by dense population regions, or if the target cells are spatially located far away from drug source points, here blood vessels. To study how a heterogeneous micro-environment impacts drug resistance and drug response, blood vessels are non-equidistantly located across the in silico domain. Moreover, the speed of molecules such as oxygen, drugs and exosomes, depends on the medium that the molecules in question are traversing [6, 109]. This section provides information regarding the modelling of various drug resistant categories. Numerical values of the parameters introduced in this section are listed in the Section 3.3.4.5, along with schematic representations of algorithms used in the model and a sensitivity investigation of critical parameters which demonstrates that our results are robust in regards to these parameters. Thus our qualitative findings, concerning drug response in cancer cell populations hosting various types of drug resistance, hold for variations of the chosen parameters. 


\section{DRUG RESISTANCE}

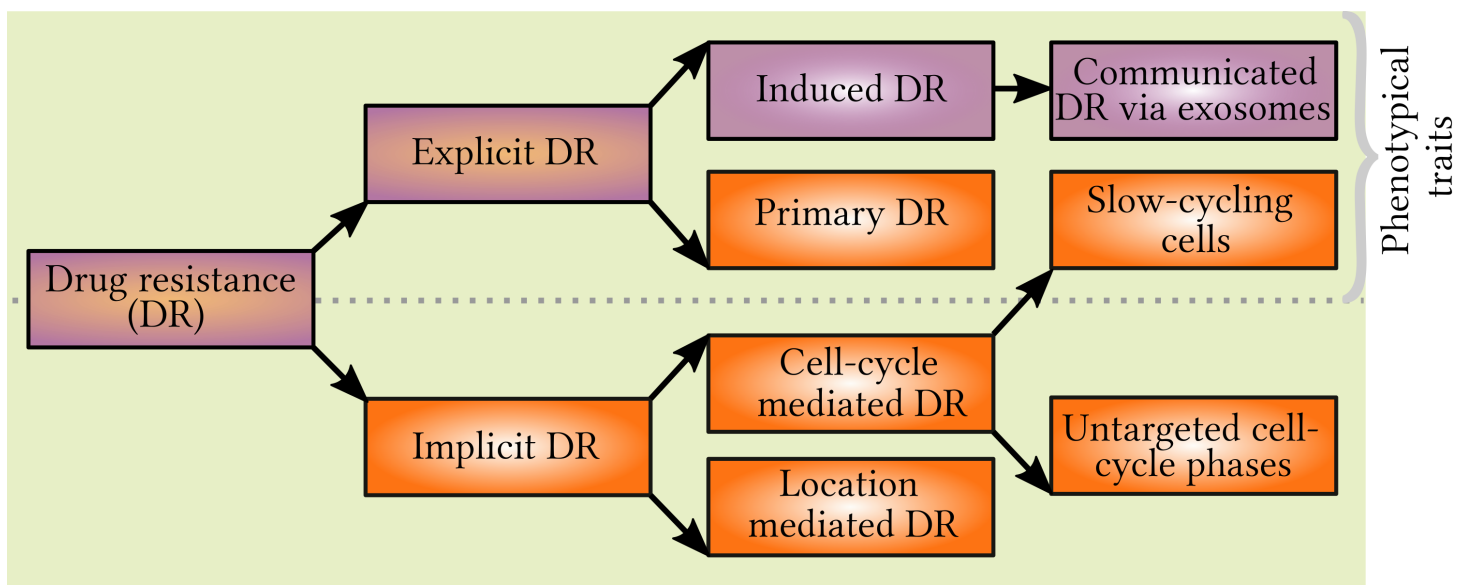

Figure 3.2: Classification of drug resistance categories occurring in the model. Drug resistance may be independent of drug presence (orange labels), induced as a consequence of drug presence (purple labels), or either (orange and purple labels). Cell acquisition of any DR phenotypical trait (top half) is here modelled as irreversible and inheritable by any future daughter cells to the cell in question.

\subsubsection{Primary Drug Resistance}

Cells display primary drug resistance independently of drug presence, thus primary drug resistance may precede chemotherapy [95, 115, 154]. The in silico setup in our study is analogous to Luria and Delbrück's [115] in vitro experiment, however here bacteria and virus have been exchanged for cancer cells and chemotherapy drugs respectively. Luria and Delbrück [115] stated that there was a probability per unit time that a sensitive cell would mutate into a, here drug, resistant phenotype. Thus in accordance with Luria and Delbrück models [115], primary explicit drug resistance is here modelled by stochastic cell mutations occurring at cell division. More specifically the chance of mutation is given by the mutation rate $\alpha_{p r i}$, which corresponds to the probability of mutation per cell cycle. The parameter $\alpha_{\text {pri }}$ is assumed to be small, so that drug resistant mutations are rare and moreover the probability that a mutated cell will revert back to a sensitive state is negligible and set to zero [115]. Hence, in our model, each daughter cell that is produced has a chance $\alpha_{\text {pri }}$ of being explicitly drug resistance before being placed on the grid. 


\subsubsection{Induced Drug Resistance}

Drug presence has been demonstrated to speed up the development of DR subpopulations in cancers [116] and multiple studies have shown that cancer cells display altered epigenetic features following chemotherapy [153]. Cancer cells may become drug resistant after exposure to chemotherapy, the underlying cause of such induced drug resistance is assumed multifactorial [154]. Factors that may contribute to induced drug resistance include decreased apoptotic response, increased DNA-repair post drugmediated damage, cell cycle alterations and reduced drug accumulation [154]. These factors may work concurrently and jointly towards establishing drug resistance in cells [154]. Hsps aid signalling pathways promoting cell growth and sustainability [99], and may induce anti-apoctic cancer cell properties [117]. Three members of the Hsp family that have been under scrutiny are namely Hsp27, Hsp70 and Hsp90, which have all been linked to promoting breast cancer tumours [99] and shown in vivo to contribute towards chemotherapeutic drug resistance [101]. In cancer cells, Hsps are plentiful and moreover administration of chemotherapeutic drugs has been observed to alter Hsp expression and increase Hsp activity [117]. Hsps may reside in both the cytoplasm and the cell nucleus, Vargas-Roig et al. demonstrated in vivo that chemotherapy drugs may modify the proportion of Hsps in different cell compartments [101], in fact drug administration resulted in increased nuclear expression, and decreased cytoplasmic expression of Hsp27 and Hsp70 [101]. Hsp27 promotes cell migration and differentiation in vivo [99] and elevated Hsp27 levels have been correlated to doxorubicon resistance and correspond to high tumorigenicity whilst low Hsp27 levels suppress tumour functions such as angiogenesis and proliferation of endothelial cells [99]. Hsp70 is linked to tumour growth and yields anti-apoptotic effect in tumours [99] and in breast cancer cells, a high proportion of nuclear Hsp70 is correlated to drug resistance [101]. Hsp90 is associated with regulating the cell cycle and controlling metastasis and proliferation [99] and high Hsp90 levels are linked to decreased survival rates in breast cancer patients [99]. Inhibiting Hsp27, Hsp70, Hsp90 has been hypothesised as part of future treatment plans [117, 118] and moreover Hsp70 has been suggested as a factor to prognostically evaluate the risk of disease recurrence [99]. The heat shock factor 1 protein (HSF1) regulates Hsps [118, 156], and HSF1 overexpression is linked to poor prognosis in breast, lung and colon cancers, an increase in intratumoural cancer stem cells proportions and chemotherapeutic drug resistance [156]. Specifically, cells with high HSF1 levels have 


\section{DRUG RESISTANCE}

displayed increased paclitaxel resistance [156]. Although cellular Hsp expression and activity have been linked to anti-cancer drug resistance, the details of these mechanisms are yet to be elucidated [101]. Thus in the mathematical model discussed in this chapter, details concerning such underlying mechanisms of acquired drug resistance are omitted and we simply recognise the fact that if a cell has been exposed non-lethal drug levels for a certain amount of time, it can develop resistance to that drug. Furthermore, clinically, cancers are usually treated with combination therapies which makes it difficult to deduce rigorous information regarding how induced drug resistance is developed in cells as a response to one particular drug [157]. Due to this multifactorial nature of induced drug resistance, which involves various subcellular alterations occurring in response to drug presence in the micro-environment, in the model a cell obtains induced explicit drug resistance once it has been exposed to a high enough drug concentration for a sufficiently long time. Thus if a cell has experienced a minimum drug concentration $\chi_{\text {ind }}$ for $\tau$ time units, drug resistance is induced in the cell.

\subsubsection{Communicated Drug Resistance via Exosomes}

Srinivasan et al. [109] investigated exosome kinetics in lymphatic flow in vitro and in vivo using near infrared imaging. In the in vitro study, they found that planted exosomes from the HEY cell line, being spherical with a diameter of around $70 \mathrm{~nm}$, travelled more effectively than size and density matched beads across lymphatic endothelial cells (LECs). This indicates that exosomes travel purposefully as opposed to randomly. Srinivasan et al. [109] reported an effective permeability for exosomes across the lymphatic endothelium in the order of $0.2 \mu \mathrm{m} / \mathrm{s}$ and moreover exosomes were transported twice as fast across areas with cells compared to areas with no cells. Exosomes were observed to move rapidly in vivo, indeed they travelled $10 \mathrm{~cm}$ in a mouse tail within 2 minutes [109]. Studies also show that there is a correlation between the micro-environment and exosome activity, as exosome secretion and uptake is promoted in low-pH regions [120]. Further, hypoxic regions are associated with drug resistance [158] which is partly explained by elevated exosome secretion in such regions [123]. Here, exosomes are modelled using phenomenological rules formulated from experimental observations, incorporating stochasticity. Exosomes are modelled as discrete 'molecule parcels'. Once per cell cycle each cell that has acquired drug or exosome induced drug resistance has a chance $\alpha_{e x}$ of producing and secreting such a molecule parcel which is 
sent off in a random direction. The first sensitive cell that the exosome hits is dubbed the recipient cell, upon receiving the exosome the recipient consumes the parcel to gain drug resistance. Exosome production and uptake times are incorporated in the travel time and thus modelled as instant, using data from Srinivasan et al. [109], we choose a propagation speed of $0.2 \mu \mathrm{m} / \mathrm{s}$ which is equivalent to 1 grid point $(20 \mu \mathrm{m})$ per $100 \mathrm{~s}$ when travelling across cells, and half of this speed when travelling across extracellular space. The chance of exosome production $\alpha_{e x}$ is significantly higher in hypoxic regions in order to account for increased exosome activity in such regions [123].

\subsubsection{Cell Cycle Mediated Drug Resistance by Slow-Cycling Cells}

Slow-cycling cells are distinguishable in vitro [119] and Srinivasan et al. [110] demonstrated that fast and slow-cycling cells may coexist in a tumour. Many chemotherapeutic drugs, such as cisplatin, attack only cells in a certain phase of the cell cycle by targeting proteins overexpressed in the corresponding phase, leaving other cells unaffected [6, 148]. Since the half-life times of common chemotherapy drugs are significantly shorter than the average cell cycle length of standard eukaryotic cells [6], slow-cycling cells are implicitly more resistant to chemotherapy as they are likely to evade drug impact whilst being in a prolonged untargeted cell cycle phase. Consequently, if there exists a subpopulation of slow-cycling cells in a tumour, this subpopulation is more likely to survive chemotherapy and proliferate post treatment, despite having a slower production rate. Such a slow-cycling subpopulation may comprise CSC-like cells, as slow-cycling cells have been linked to cancer stem cells [96], which in turn have been conferred with reduced sensitivity to chemotherapeutic drugs [156]. Previous research indicates that cancer cells may obtain stem-cell like traits [159], in fact non-stem cancer cells may convert into CSC-like cells seemingly spontaneously, as demonstrated in vivo by Chaffer et al. [160]. Micro-environmental factors, such as oxygen supply, may influence such conversions however here cell conversion into a slow-cycling state is modelled as independent of the micro-environment. The $[\mathrm{HIF}]$ parameter, occurring in Equation 2.1, which is switched on in hypoxic cells only does however increase the cell cycle length of all cells, fast-cycling and slow-cycling, and thus in the model, oxygen levels effect cell-cycle lengths. Slow-cycling cells are multidrug resistant as they are resistant to any drug that targets only a subset of cell cycle phases. In the model, we assume that slow-cycling conversion occurs spontaneously, independently of drug presence. More 


\section{DRUG RESISTANCE}

specifically, once per cell cycle there is a chance $\alpha_{S C}$ that a sensitive, fast-cycling cell will spontaneously convert into a slow-cycling state. Here slow-cycling cells are assumed to have a cell cycle length that is roughly twice as long as a sensitive cell [132]. The chance of conversion from a slow-cycling, implicitly drug resistant state back into a fast-cycling state is assumed negligible in accordance with previously discussed Luria Delbrück models [115] and Darwinian principles and thus set to zero. Furthermore, the in silico experiment spans 700 hours only, and the chance that a cell converts and re-converts is assumed negligible in the model. Once a cell is randomly selected to convert into a slow-cycling state, the individual growth rate factor $\mu$ of the regarded cell, occurring in Equation 2.1, is updated in order to achieve slower cell-cycle progression and by extension a longer cell-cycle length. Daughter cells to slow-cycling cells are assigned appropriate growth rates associated with slow-cycling cells at creation. In the model, slow-cycling cancer cells differ from normal cancer cells in that their value $\mu$ in Equation 2.1f is doubled.

\subsubsection{Parameters and Algorithms for Drug Resistance}

The chosen parameter values used for simulating drug resistance in the in silico framework are listed in Table 3.3 , where in hypoxic regions $\alpha_{e x}$ is increased to $2 \alpha_{e x}$.

\begin{tabular}{c|c|c|c|c}
$\alpha_{\text {pri }}$ & $\chi_{\text {ind }}$ & $\tau$ & $\alpha_{\text {ex }}$ & $\alpha_{S C}$ \\
\hline 0.01 & $\kappa / 10$ & 30 minutes & 0.07 & 0.07
\end{tabular}

Table 3.3: Parameters concerning drug resistance used in the in silico framework. $\alpha_{p r i}$ denotes the probability that a cell that is placed on the lattice is drug resistant (when simulating scenarios involving primary drug resistance). $\chi_{\text {ind }}$ and $\tau$ respectively denote the drug concentration and time required to induce drug resistance in a cell (when simulation drug induced drug resistance). $\alpha_{e x}$ denotes the probability that a drug resistant cell will produce an exosome that induces drug resistance in a nearby cell (when simulating communicated drug resistance). $\alpha_{S C}$ denotes the probability that a cell will convert to a slow-cycling state (when simulating slow-cycling mediated drug resistance).

The results in this study are qualitative, when varying the parameters in this table, as done in Appendix A1, the obtained qualitative results are robust in regards to chemotherapy response. Here, $\tau$ is chosen to be 30 minutes as this is close to the half-life time of cisplatin. $\alpha_{p r i}, \chi_{i n d}, \alpha_{e x}$ and $\alpha_{S C}$ are parameterised to be low and yield approximately the same ratio between the sensitive subpopulation and the drug resistant subpopulation, across all the different investigated in silico scenarios, 10 hours after the first drug administration when the lowest drug dose is applied. (In other words, all tests 
named (b1), (c1), (d1) and (e1), according to the labelling system declared in Tables 3.4 and 3.5 , comprise roughly the same composition of sensitive and drug resistant cells 10 hours post drug administration). This is in order to allow for easy comparisons, in regards to drug response evaluations, amongst tests. Partial algorithms for incorporating various types of drug resistance in our mathematical framework are listed below.

Primary drug resistance: Each time cell division occurs there is a chance that a drug resistant cell will be produced. In the model, when cell division occurs it is said that a mother cell produces a daughter cell. If the mother cell is drug resistant (DR), the daughter cell will inherently be drug resistant. However, if the mother cell is sensitive (S), the daughter cell may or may not be drug resistant according to a stochastic 'dice roll'. At each cell division, a value $\alpha \in[0,1]$ is randomised, and if $\alpha \leq \alpha_{p r i}$ then the daughter cell is drug resistant, otherwise it is sensitive, as schematically shown in Figure 3.3 .

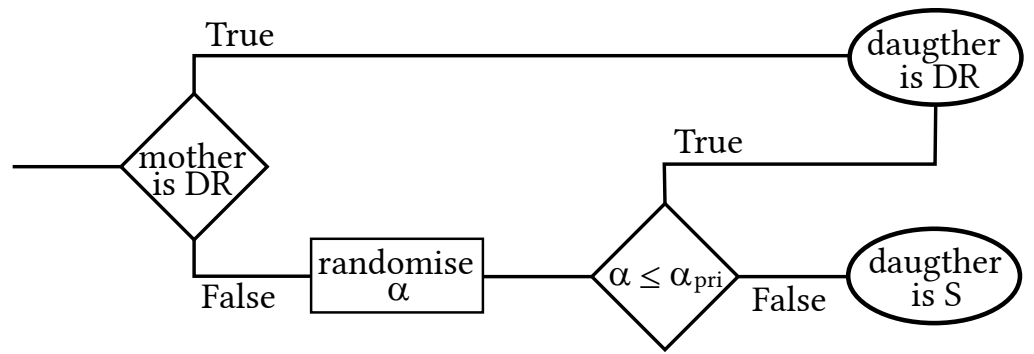

Figure 3.3: Partial algorithm for determining primary drug resistance.

Induced drug resistance: If a cell has experienced a minimum drug concentration $\chi_{\text {ind }}$ for $\tau$ time units, drug resistance is induced in the cell. Thus in the model, each cell has its own counter, counter $_{n}$, which increments each time step that cell ${ }_{n}$ experiences a drug concentration $\chi=\mathscr{C}(x, t) \geq \chi_{\text {ind }}$. This is schematically illustrated in Figure 3.4. If drug resistance has been produced in a mother cell, it produces drug resistant daughter cells.

Communicated drug resistance via exosomes: Once per cell cycle, each cell that has acquired drug resistance by induced drug resistance (see the above section) or communicated drug resistance (as described in this section) has a chance $\alpha_{e x}$ of producing and secreting an exosome. In the model, a value $\alpha \in[0,1]$ is randomised, if $\alpha \leq 2 \alpha_{e x}$ in hypoxic regions, or $\alpha \leq \alpha_{e x}$ in normoxic or hyperoxic regions, an exosome 


\section{DRUG RESISTANCE}

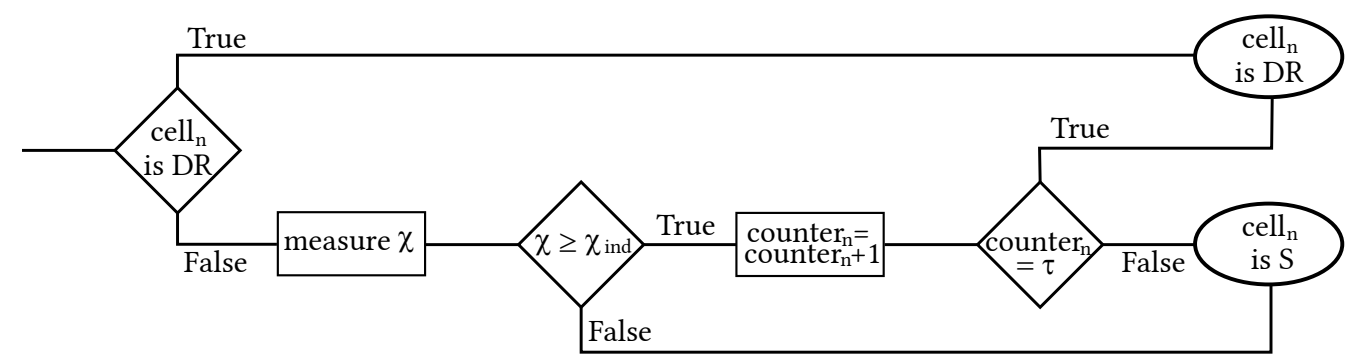

Figure 3.4: Partial algorithm for determining induced drug resistance.

is produced and sent off in a random direction. The first sensitive cell that the exosome hits becomes drug resistant, as schematically shown in Figure 3.5. Mother cells that have acquired drug resistance via exosomes pass on their drug resistance to daughter cells.

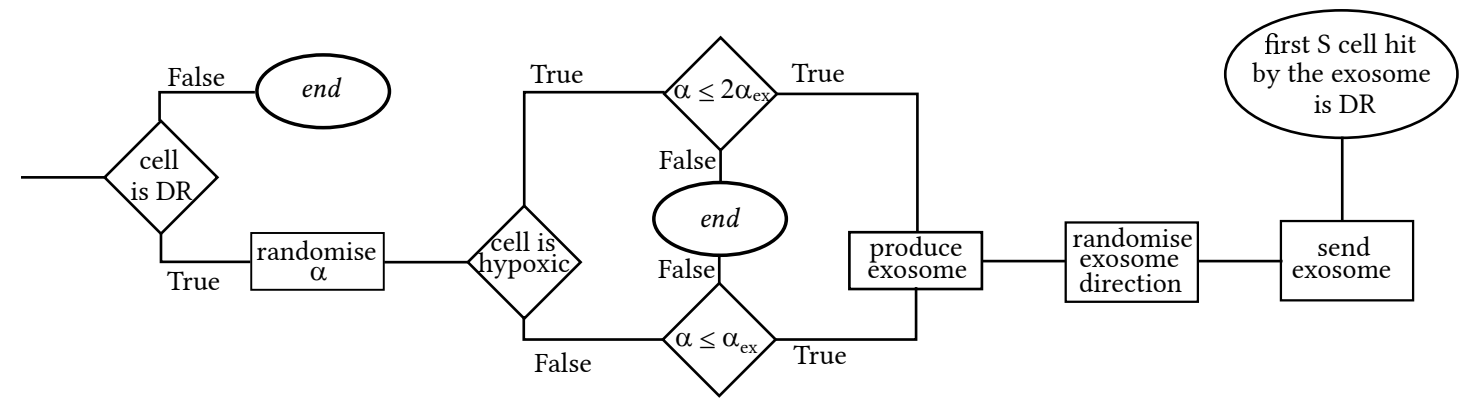

Figure 3.5: Partial algorithm for determining exosome production.

Cell-cycle mediated drug resistance by slow-cycling cells: Once per cell cycle there is a chance that a sensitive, fast-cycling (FC) cell will spontaneously convert into a slow-cycling (SC), drug resistant state. To check if such conversion occurs, a value $\alpha \in[0,1]$ is randomised. If $\alpha \leq \alpha_{S C}$ then the cell converts, otherwise it does not, as demonstrated in Figure 3.6. Slow-cycling (SC DR) mother cells yield slow-cycling (SC DR) daughter cells.

\subsubsection{Implementation}

The $\mathrm{CA}$ is implemented in $\mathrm{C} / \mathrm{C}++$ using a high performance computational framework. Ordinary differential equations are solved using a fourth order Runge-Kutta method and partial differential equations are solved using explicit second-order finite difference schemes. A flowchart of the programming code is available in Appendix A1. Prior to 


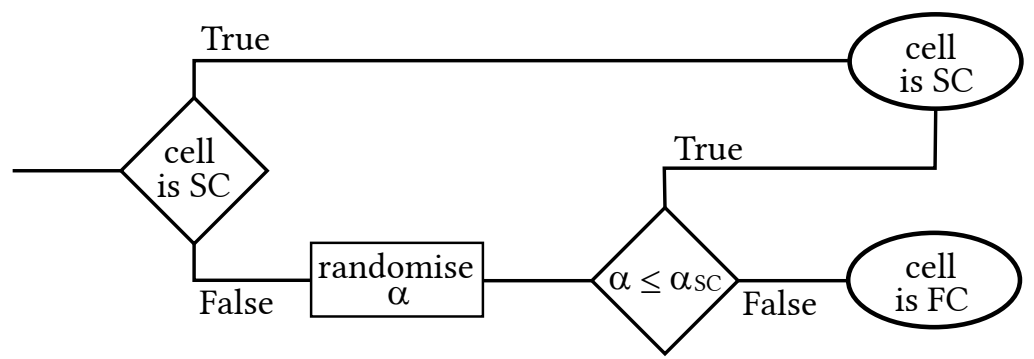

Figure 3.6: Partial algorithm for determining the spontaneous conversion from a slowcycling to a fast-cycling state.

commencing the in silico experiment, the micro-environment is implemented and thus blood vessel cross sections are scattered in a non-equidistant fashion across the grid, this is in order to emphasise and study the effects of spatio-temporal oxygen and drug heterogeneity. One initial cancer cell is then planted at the centre of the grid, from this originating cell the cancer cell population will grow. Tumour growth is simulated over 700 hours, where it has been concluded that 700 hours is a sufficiently long simulation time to study the drug resistance and drug response in the system. Here, a time step size $\Delta t=10^{-3}$ hours, is used in accordance with the appropriate nondimensionalisation of the oxygen parameters occurring in Equation 3.1 [6]. Cisplatin, a chemotherapy drug which here is modelled to attack G1 cells only, is administered in two instances at 500 and 600 hours. For each such instance, drugs are produced on all blood vessels at one single time step. The total amount of drug produced at one time step, on all blood vessels, corresponds to the drug dosage, which can be varied to study various cases. Specifically, here the studied drug dosage are: (1) 1C, (2) 2C, (3) 4C or (4) 8C, as listed in Table 3.5. The dosage $1 \mathrm{C}$ is parametrised so that, in the absence of drug resistant phenotypes amongst the cells, $1 \mathrm{C}$ kills half of the total cell population shortly after the first drug administration at 500 hours. By doubling the drug dosage once, twice or thrice to $2 \mathrm{C}, 4 \mathrm{C}$ or $8 \mathrm{C}$ respectively a higher cell kill is achieved. However, the relationship between the drug dosage and the number of cells killed is not linear, as is further discussed in Section 3.4.1.1. For the fixed chemotherapy schedule at 500 and 600 hours, the administered drug dosages are varied and the size of the sensitive and drug resistant cell subpopulations are computed pre, peri, and post chemotherapy. Cell-maps are produced in order to display cell population topology over time, these cell-maps are visualised in ParaView [161]. Five different in silico experiments are here performed, corresponding to five different categories of drug resistance namely (a) to (e) listed in 


\section{DRUG RESISTANCE}

\begin{tabular}{ll}
\hline Label & Drug Resistance Category \\
\hline $\mathrm{a}$ & No drug resistance (No DR) \\
$\mathrm{b}$ & Primary drug resistance (Primary DR) \\
$\mathrm{c}$ & Induced drug resistance (Induced DR) \\
$\mathrm{d}$ & Induced and, via exosomes, communicated drug resistance (ICC DR) \\
$\mathrm{e}$ & Cell cycle mediated drug resistance by spontaneous conversion to slow- \\
& cycling cells (SC DR) \\
\hline
\end{tabular}

Table 3.4: The labelling of drug resistance categories occurring in the model.

\begin{tabular}{ll}
\hline Label & Drug dosage \\
\hline 0 & $0 \mathrm{C}$ (No drug) \\
1 & $1 \mathrm{C}$ \\
2 & $2 \mathrm{C}$ \\
3 & $4 \mathrm{C}$ \\
4 & $8 \mathrm{C}$ \\
\hline
\end{tabular}

Table 3.5: The labelling of drug dosages used in the implementation. For non-zero drug dosages the labelling system is such that label $\ell$ corresponds to drug dosage $2^{\ell-1}$.

Table 3.4 Each experiment is performed 100 times, subsequently average values and standard deviations are reported. A sensitivity investigation of critical parameters is performed in order to confirm that our results are robust in regards to small parameter variations, this is available in the Appendix A1.

\subsection{Results and Discussion}

\subsubsection{Results}

In absence of chemotherapeutic drugs, the growth of the DR subpopulation in our in silico experiment is proportional to the growth of the $\mathrm{S}$ subpopulation, as demonstrated by the graphs in Figure 3.7. This is in accordance with previous mathematical models and experimental results [95, 106, 115].

\subsubsection{No Drug Resistance}

Once chemotherapy is applied to the in silico setup, the cancer cell population decreases in response to drugs. This is especially clear when no drug resistant phenotypes exist, the cancer cell population then rapidly reduces after drug administration. However population growth quickly resumes, and the size of such populations eventually reaches, 

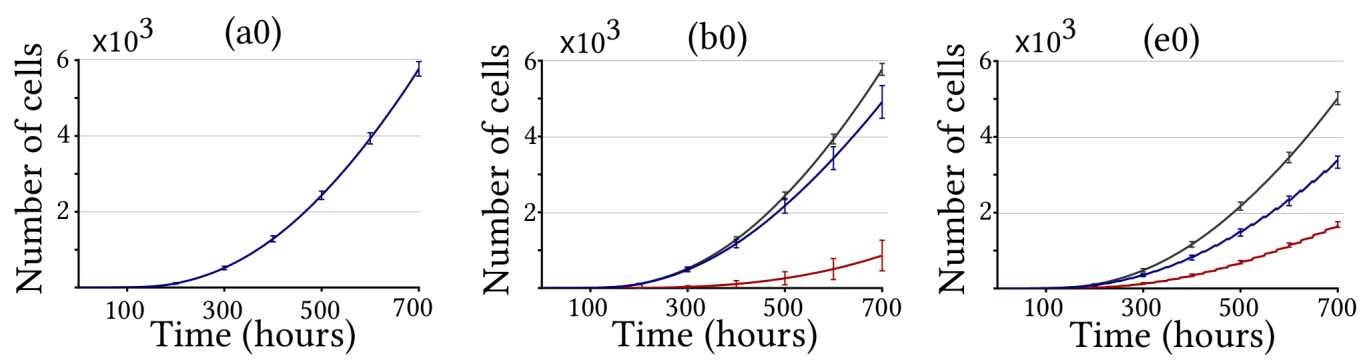

Figure 3.7: Growth of the cancer cell population over time in drug absence, showing total population (black graph), drug sensitive subpopulation (blue graph) and drug resistant subpopulation (red graph) incorporating control-case drug resistance categories (a0) No DR, (b0) Primary DR and (e0) Slow-Cycling DR. Cases (c0) Drug-induced DR and (d0) Communicated (ICC) DR are omitted since they produce the same results as (a0) in drug absence. Each graph shows the average number of cells based on 100 simulations, the standard deviation is demonstrated with error bars.

and surpasses, the pre-chemotherapy population size. This is evident in graphs (a1) through to (a4) in Figure 3.8, and especially clear for high chemotherapy dosages where the size of the cell population cycles between being large immediately before drug administration and being small directly after drug administration. Rottenberg and Borst [98] demonstrated similar cyclic behaviour in mouse tails in vivo. Increasing the drug dosage trivially kills more cancer cells, however tumour topology and spatial heterogeneity significantly affects drug transport. Moreover, due to intratumoural heterogeneity in regards to the cell cycle, some cells will be shielded from the drug simply by being in an untargetted cell cycle phase. This multiscale heterogeneity affects the relationship between drug dosage and drug efficacy, where drug efficacy here corresponds to the number of cells killed by the drug. Graphs (a1) to (a4) in Figure 3.8 demonstrate the poor scaling between drug dosage and drug efficacy. Further, here we define the scaling efficiency $\epsilon$ by

$$
\epsilon_{i}=\frac{k_{i}}{k_{1} \cdot N_{i}},
$$

where $k_{i}$ denotes the number of cells killed in experiment $i$ and $N_{i}$ denotes the dimensionless drug dosage coefficient used in experiment $i$. Thus here, $i=1,2,3,4$ and respectively $N_{i}=1,2,4,8$. If the relationship between drug dosage and drug efficacy was linear, doubling the drug dosage would mean doubling the number of cancer cells killed. In such an ideal case, it would hold that $\epsilon_{i}=1 \forall i$, however this is not the case, as illustrated in Figure 3.9 which shows the scaling efficiency for the first drug administration at 500 hours. Hence in a clinical setting, the harm that may follow increased 


\section{DRUG RESISTANCE}

toxicity from higher drug dosages may not be worth the modest increase in killed cancer cells. The cell-maps in Figure 3.10 further highlight how the spatial heterogeneity affects drug delivery and consequently where the drug concentrations are high enough for cell kills to occur.

\subsubsection{Primary Drug Resistance}

When a cancer cell population expresses primary drug resistance, two subpopulations, comprising sensitive and drug resistant cells, already coexist prior to chemotherapy. Once administered, chemotherapy eliminates cells belonging to the $\mathrm{S}$ subpopulation whilst leaving the DR subpopulation unharmed. Hence increasing the drug dosage, and consequently killing more sensitive cells, enables the DR subpopulation to flourish as it gains access to more resources such as space and oxygen. Graphs (b1) through to (b4) in Figure 3.8 show that such DR subpopulations indeed benefit from high drug environments. Over all, our results show that chemotherapeutic treatments yield poor results if there exists a subpopulation of drug resistant cells prior to commencing treatment, this supports clinical observations depicting that primary drug resistance gravely reduces the successfulness of chemotherapy and influences the choice of treatment regime [93]. In our experiment, the primary DR subpopulations grow outward in radial strands pre chemotherapy, as illustrated in cell-maps (b1) and (b4) in Figure 3.10. This geometry is explained by the model, in which primary drug resistant mutations occur at cell division, prior to placing the cell on the grid. Moreover drug resistant cells produce drug resistant offspring, thus radial strands are formed as the cancer cell population grows. This DR subpopulation will spread from these strands, which consequently will widen post drug administration. On the other hand, subpopulation S will spread from regions containing sensitive cells that survived drug effect. Such sensitive survivor cells are clustered in regions of low drug concentrations, typically located far away from blood vessels and enclosed by other cancer cells, as drugs travel more slowly over dense population regions.

\subsubsection{Induced Drug Resistance}

For simulations incorporating induced drug resistance, the DR subpopulation arises post chemotherapy. Here, drugs diffuse more slowly over cancer cells than over extracellular space. Therefore, once the initial effect of chemotherapy has eliminated the majority 

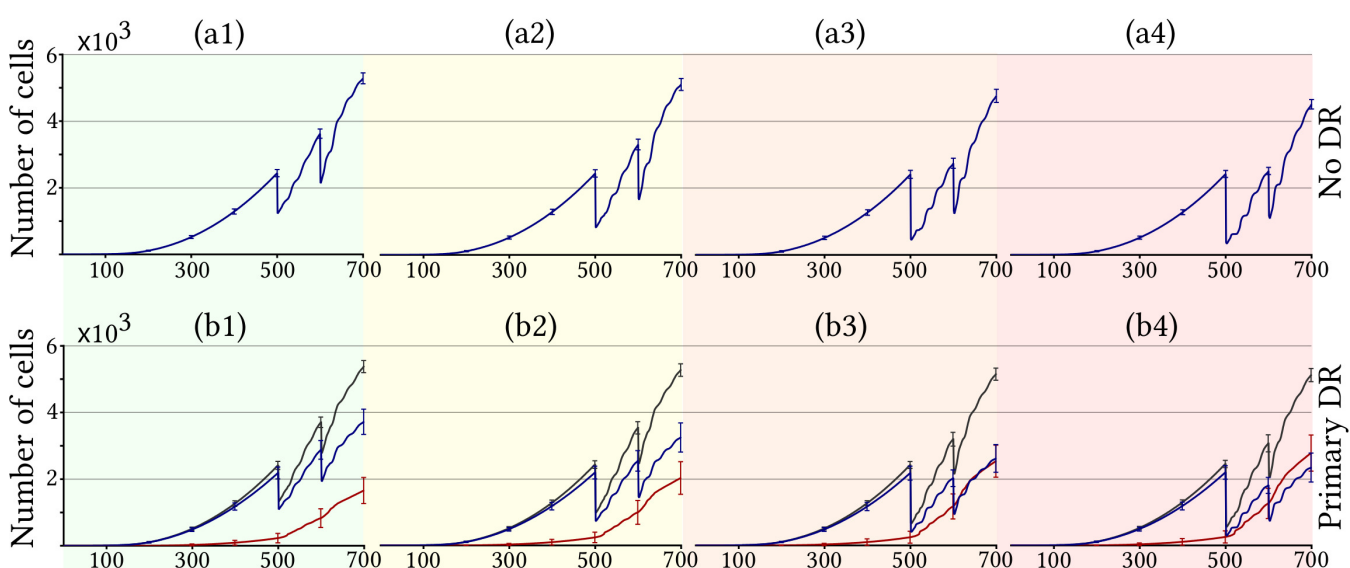

(b2)

(b3)

(b4)
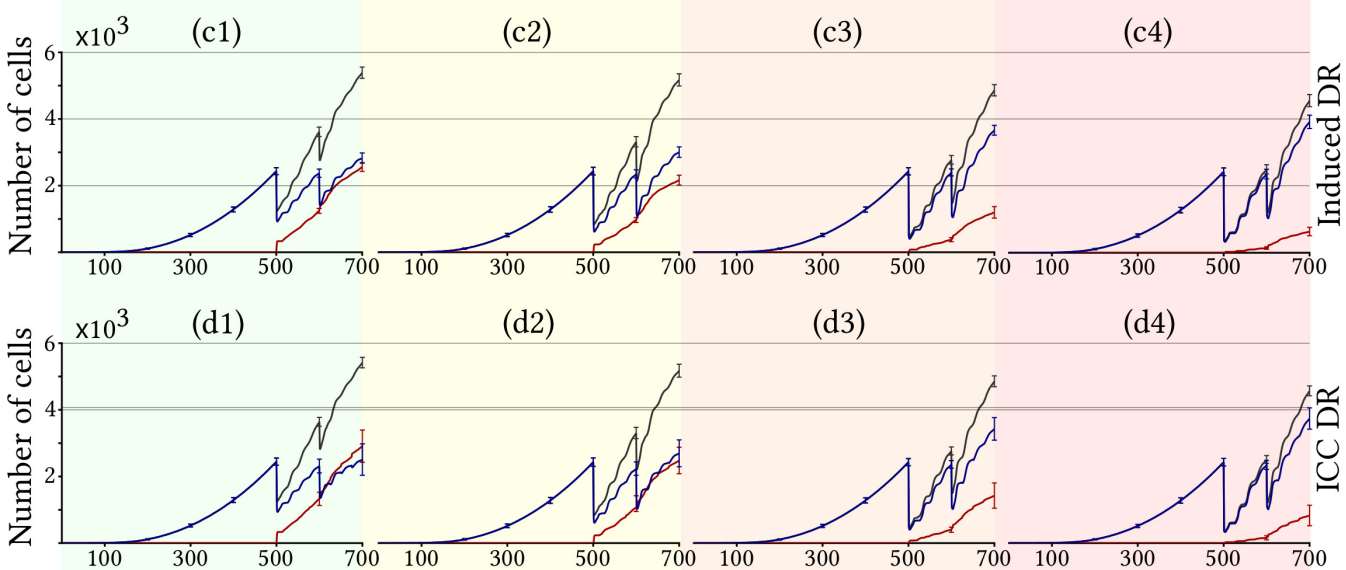

(d2)

(d3)

(d4)

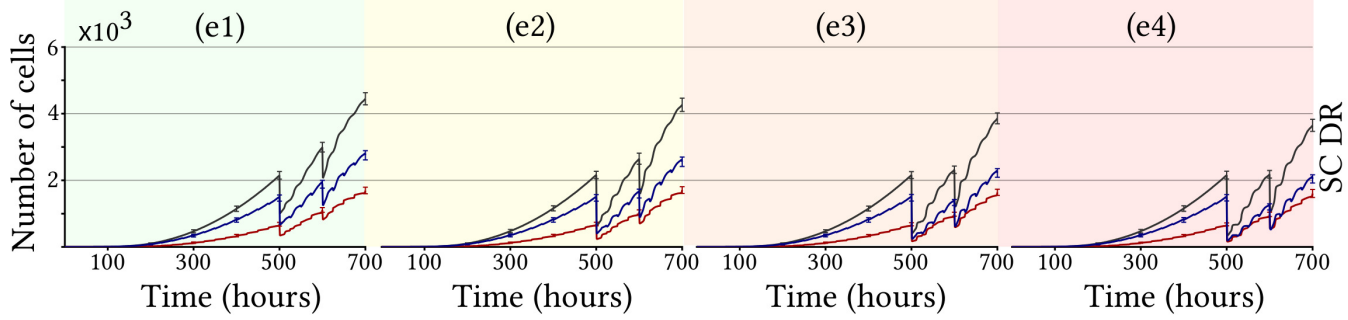

Figure 3.8: Growth of the cancer cell population over time when drugs are applied at 500 and 600 hours, showing total population (black graph), drug sensitive subpopulation (blue graph) and drug resistant subpopulation (red graph). Each row in the figure corresponds to a category of drug resistance (a) to (e) according to the labelling in Table 3.4 ((a) No DR, (b) Primary DR, (c0) Drug-induced DR, (d0) Communicated DR (e) Slow-cycling DR), whilst each column corresponds do a specific drug dosage varying from low (leftmost column) to high (rightmost column), namely (1) 1C, (2) 2C, (3) 4C and (4) 8C according to the labelling system in Table 3.5. Each graph shows the average number of cells based on 100 simulations, the standard deviation is demonstrated with error bars. 


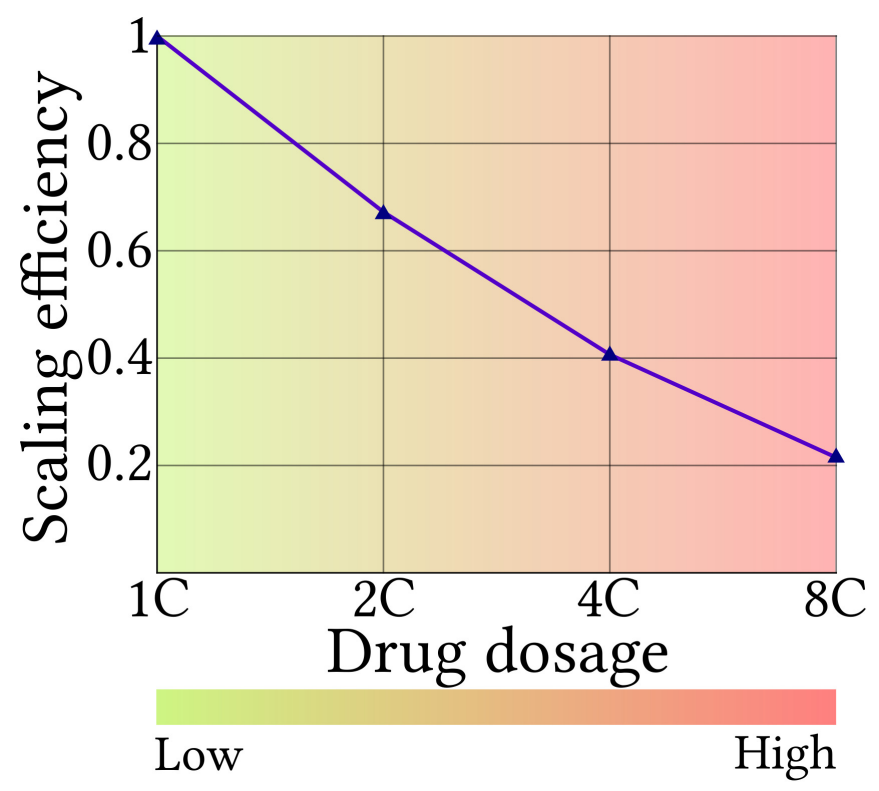

Figure 3.9: The scaling efficiency, demonstrating the relationship between drug dosage and drug efficacy in terms of number of killed cancer cells. This is for the first drug administration at 500 hours for experiments (a1) to (a4) according to the labelling in Tables 3.4 and 3.5 where no drug resistant phenotypes are present. Results are based on average values for 100 tests.

of the sensitive cancer cells, and the tumour has disintegrated into clusters of surviving cancer cells, drug resistant cells are typically located on boundaries between cancer cells and extracellular space, as illustrated in cell-maps (c1) and (c4) in Figure 3.11 On these boundaries drug resistance is induced since the cells are exposed to high, but non-lethal, drug concentrations for a sufficiently long time. From these points of origin the DR subpopulation spread. By increasing the amount of chemotherapy, the cells that are exposed to this intermediate, high but non-lethal, drug concentration is reduced. Hence higher chemotherapy dosages do not only kill more cells overall, but reduces the amount of drug resistant phenotypes, as demonstrated by graphs (c1) to (c4) in Figure 3.8, this result applies to a cancer cell population in a confined space.

\subsubsection{Communicated Drug Resistance via Exosomes}

The effect of ICC is demonstrated by comparing experiment (c) Induced drug resistance, to experiment (d) Induced and, via exosomes, communicated drug resistance. This can be done by regarding graphs $(\mathrm{c} 1)$ to $(\mathrm{c} 4)$ and $(\mathrm{d} 1)$ to $(\mathrm{d} 4)$ in Figure 3.8 and cellmaps (c1), (c4), (d1), (d4) in Figure 3.11. These figures show that the communicative 
exosome component amplifies the effect of induced drug resistance alone. The number of oncogenic exosomes produced increases with the number of drug resistant cells. In low to moderate drug environments, many exosomes are thus produced, as there exists many cells which have acquired induced DR in accordance with the results in Section 3.4.1.3 Conversely, in very high drug regimes fewer cells will survive to acquire induced drug resistance and consequently fewer oncogenic exosomes will be produced. Exosomes have been hypothesised as possible treatment targets, and our results indicate that reducing the exosome activity would aid the S subpopulation, as less cells would convert from sensitive to drug resistant. Here, hypoxic cells secrete more exosomes than do normoxic cells. This results highlights one of the benefits of targeting hypoxic tumour regions, as doing so may reduce exosome activity and by extension hinder communicated drug resistance.

\subsubsection{Cell Cycle Mediated Drug Resistance by Slow-Cycling Cells}

Slow-cycling cells are more likely to evade drug effects, as shown in graphs (e1) to (e4) in Figure 3.8 where, after each drug attack, the DR slow-cycling subpopulation displays a higher survivor rate. High chemotherapy dosages increase this effect, and thus benefit the DR subpopulation. Since the conversion to slow-cycling cells is modelled as spontaneous, implicit drug resistance may precede chemotherapy. Furthermore, this spontaneity means that at every cell cycle, each fast-cycling cell has the same chance $\alpha_{S C}$ of converting to a slow-cycling state. Thus pre chemotherapy the DR subpopulation will be point-wise scattered across the cell population, and post chemotherapy the DR subpopulation will spread from these source points, as illustrated in cell-maps (e1) and (e4) in Figure 3.11

\subsubsection{Cell-Map Topology}

Each drug resistance category in our model corresponds to a typical cell-map topology and vice-versa, as shown in Figures 3.10 and 3.11 . The location of the subpopulations $\mathrm{S}$ and DR depends on the category of drug resistance regarded, as does the location of regions with high cell-kill numbers. These cell-maps are useful for conveying spatial heterogeneity, which is of importance since intratumoural heterogeneity is known to heavily influence drug efficiency [6]. Any sensitive cell, or cell cluster, that is surrounded by a band of other cells will be partly shielded from drug effects, as drugs travel slowly 


\section{DRUG RESISTANCE}

over dense population regions. Further, any quiescent sensitive cell, or cell cluster, that is surrounded by a band of explicitly drug resistant cells is eternally safe from drug presence in our in silico setup. This is because such a sensitive cell, or cell cluster, will never meet the necessary condition to re-enter the cell cycle as this requires free neighbourhood space. Thus, even sensitive cell may be protected from drug effect, this is an example of location mediated implicit drug resistance.

\subsubsection{Discussion}

Our study shows that drug response in cancer cell populations is crucially influenced by the drug resistant phenotypes amongst the cells. We here demonstrate, in silico, that the effect of chemotherapy is heavily dependent not only on the mere existence of drug resistant cells, but also the type of drug resistance displayed and micro-environmental factors. Clinically, this implies that optimal chemotherapy scheduling and dosages depend on tumour specific data, including information regarding drug resistance and tumour environment. Indeed our results show that some types of drug resistant phenotypes thrive in low drug settings, whilst other flourish in high drug settings.

Before proceeding to discuss optimal drug dosages, one must define what constitutes as "successful" chemotherapy. Is the aim perhaps to (i) reduce the cancer cell population as much and quickly as possible or to (ii) be able to control the tumour long-term using chemotherapy? Case (i) may be relevant when chemotherapy is used in combinations with other treatments, for example when neoadjuvant chemotherapy is used prior to radiation treatment or surgery. Conversely, Case (ii) may be applicable when chronic chemotherapeutic treatment strategies are used, as can be done when it is implausible to completely eliminate a tumour. In such cases it is vital to suppress any DR subpopulation in order to keep the tumour manageable by chemotherapy. For Case (i), Figure 3.12 provides the data needed to discuss intelligent treatment strategy. This diagram trivially shows that high chemotherapy dosages are the most effective to rapidly eradicate cancer cells. However, what is not shown in our result, but is of clinical importance, is that high drug dosages are coupled with high toxicity which may be harmful to patients. Moreover our results show that the relationship between drug dosage and drug efficacy scales poorly, which is worth considering in a clinical setting. The increase in toxicity following from an increased drug dosage may not validate the 
(a1)

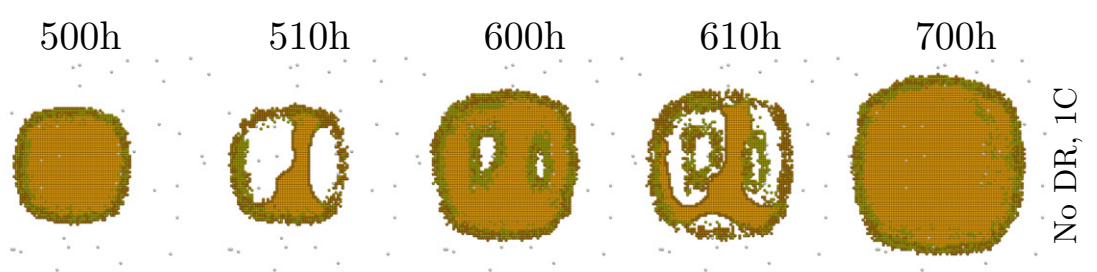

(a4)
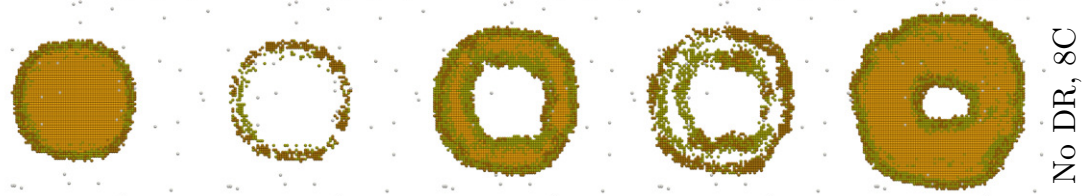

(b1)
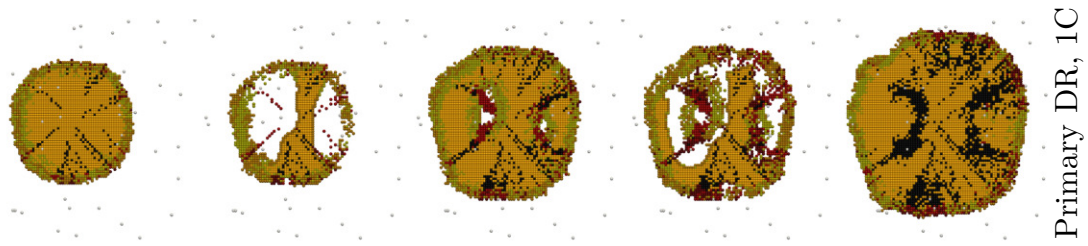

(b4)
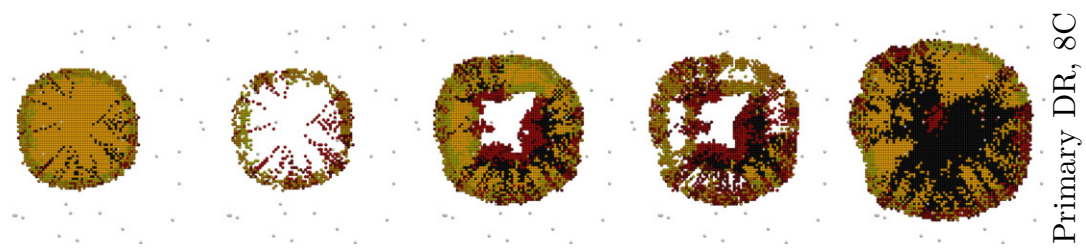

矛

:G1

S:Syn-G2-M

S:G0

DR:G1

DR:Syn-G2-M

DD:G0 Blood vessel

Figure 3.10: Cell-maps of the cancer cell populations at times $500 \mathrm{~h}$ (immediately before first drug dose), $510 \mathrm{~h} 600 \mathrm{~h}$ (immediately before second drug dose), $610 \mathrm{~h}$ and $700 \mathrm{~h}$ (end of simulation). Cases incorporating (a) No DR and (b) Primary DR are shown for drug dosages of (1) $1 \mathrm{C}$ and (4) 8C according to the labelling in Tables 3.4 and 3.5 . White areas correspond to extracellular space. 
(c1)

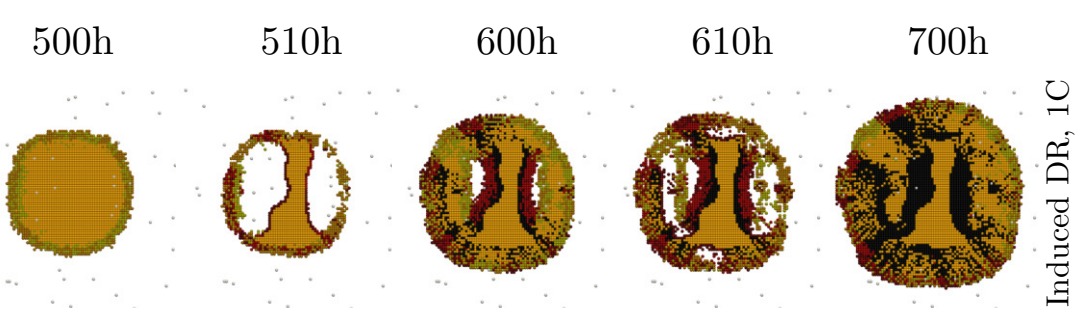

(c4)

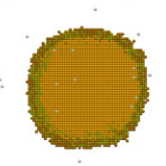<smiles>C1=CCCCCC1</smiles>
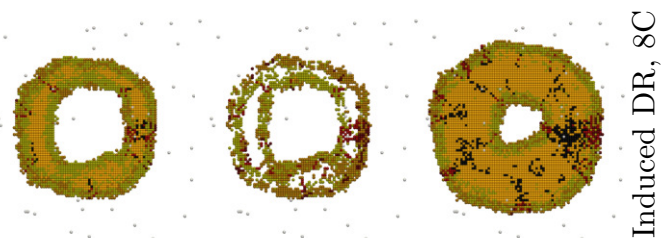

(d1)
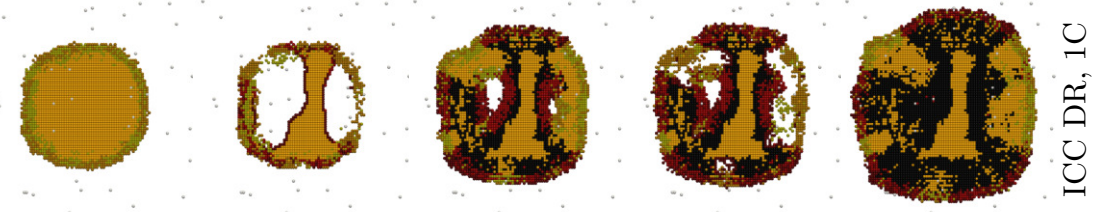

$(\mathrm{d} 4)$

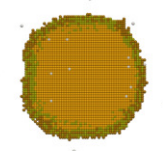<smiles></smiles>
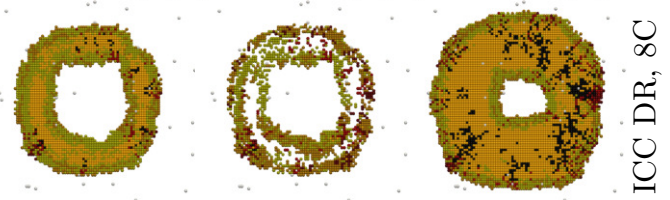

(e1)
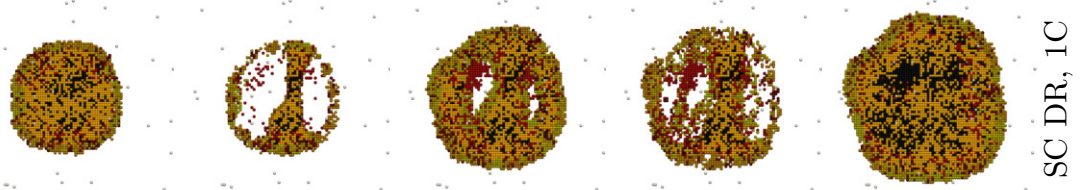

(e4)
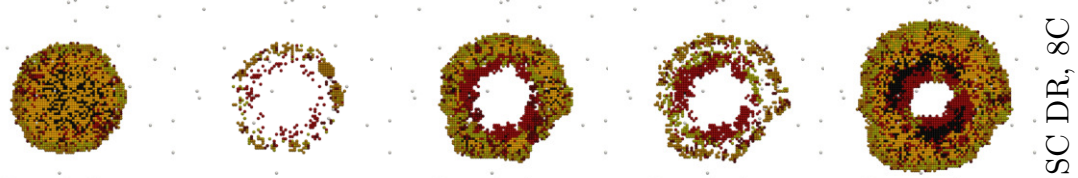

S:G1 OS:Syn-G2-M OS:G0

DR:Gi

DR:Syn-G2-M

$R: G 0$

Blood vessel

Figure 3.11: Cell-maps of the cancer cell populations at times $500 \mathrm{~h}$ (immediately before first drug dose), $510 \mathrm{~h} 600 \mathrm{~h}$ (immediately before second drug dose), $610 \mathrm{~h}$ and $700 \mathrm{~h}$ (end of simulation). Cases incorporating (c) Induced DR, (d) ICC DR and (e) SC DR are shown for drug dosages of (1) 1C and (4) 8C according to the labelling in Tables 3.4 and 3.5. White areas correspond to extracellular matrix. 
outcome in terms of tumour reduction. In Case (ii) there is a balance, and sometimes a trade-off, between eliminating sensitive cells and aiding the drug resistant cells. Indeed killing of sensitive cells paves the way for any drug resistant phenotypes. Hence administering low chemotherapy dosages sometimes constitutes a wiser treatment strategy as it delays the uprising of a drug resistant subpopulation. Evolutionary theory and the interaction between $\mathrm{S}$ and DR subpopulations may thus play a role in designing intelligent treatment strategies. Figure 3.13 shows the ratio between $\mathrm{S}$ and DR subpopulations at different times. This diagram demonstrates that drug resistant phenotypes that arise independently of drug presence benefit from high drug dosages. However, in silico, the opposite is true here for drug-induced drug resistant phenotypes, which prosper in low drug conditions. Ideal chemotherapeutic tumour treatments would involve rapidly reducing tumour size whilst minimising drug resistance, thus meeting the requirements of both Cases (i) and (ii). However, our results indicate that when using chemotherapy only, there is a trade-off between tumour reduction and the suppressing of drug resistant phenotypes in some cases, thus the objectives of Cases (i) and (ii) may conflict.

The aim of this study is to qualitatively model drug response in cancer cell populations hosting drug resistant individuals. Drug resistance is here modelled from a collective of biological experiments, biological theory and clinical observations, and thus does not confer strictly with one cell line or one experiment. However, the developed in silico framework can be parametrised and calibrated appropriately for a cell-line specific study (as shown in a recent paper [31]), should relevant biological data become available in detail. Our in silico framework is equipped to handle various mechanisms concerning drug resistance, these mechanisms can be appropriately included or excluded in order to study a certain cell-line or a certain tumour scenario.

\subsection{Conclusion}

Enhanced chemotherapeutic drug resistance post-chemotherapy is an established clinical problem, this study provides insight into drug resistance and drug response in cancer cell populations on a cellular resolution. Our results show that, whilst chemotherapy is an effective way to reduce tumours, suboptimal drug dosages may contribute towards drug resistance and, by extension, tumour reinforcement. Thus, in accordance with 


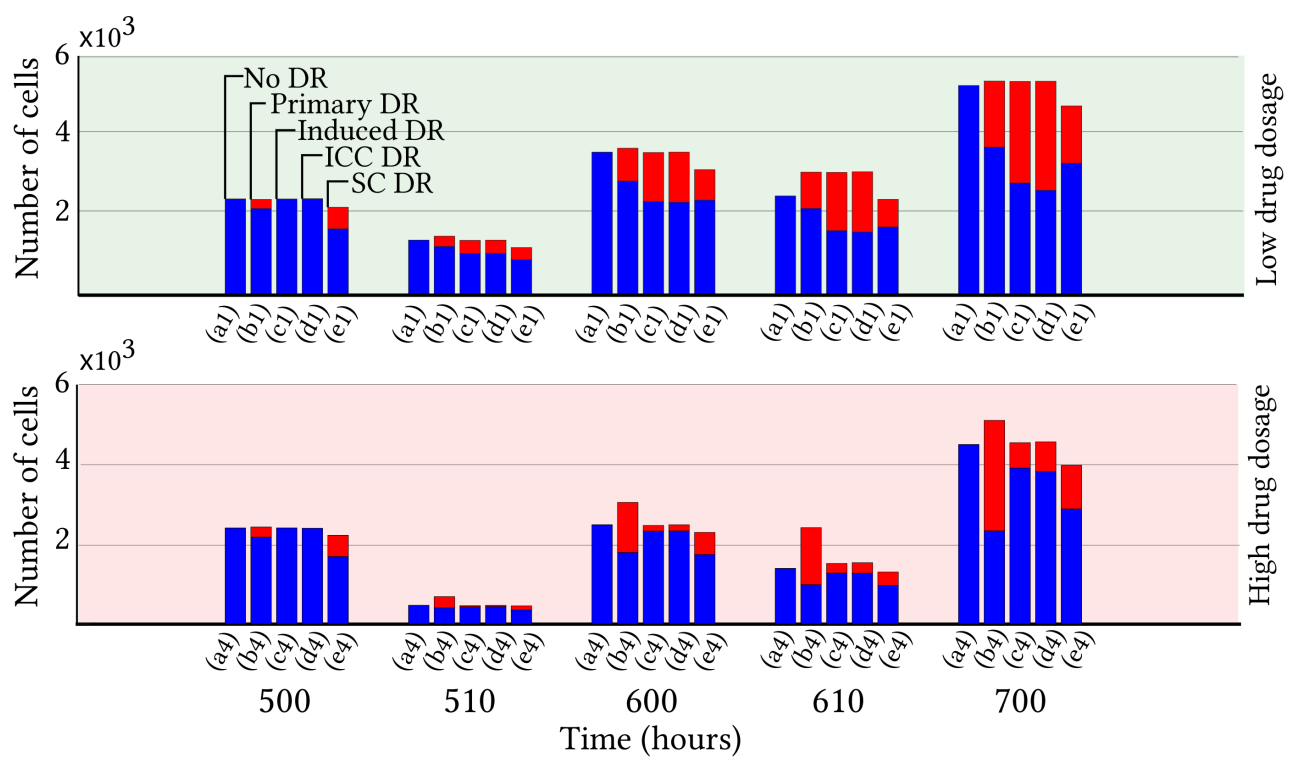

Figure 3.12: Diagram of the drug sensitive subpopulation (blue) and the drug resistant subpopulation. Various types of drug resistance are incorporated namely (a) No DR, (b) Primary DR, (c) Induced DR, (d) ICC DR, (e) SC DR. Results are shown at times $500 \mathrm{~h}$ (immediately before the first drug dose), $510 \mathrm{~h}, 600 \mathrm{~h}$ (immediately before the second drug dose), $610 \mathrm{~h}$ and $700 \mathrm{~h}$ (end of simulation) for low drug dosages, (1) 1C, and high drug dosages, (4) 8C according to the labelling in Table 3.5. Each diagram shows the average value based on 100 simulations.

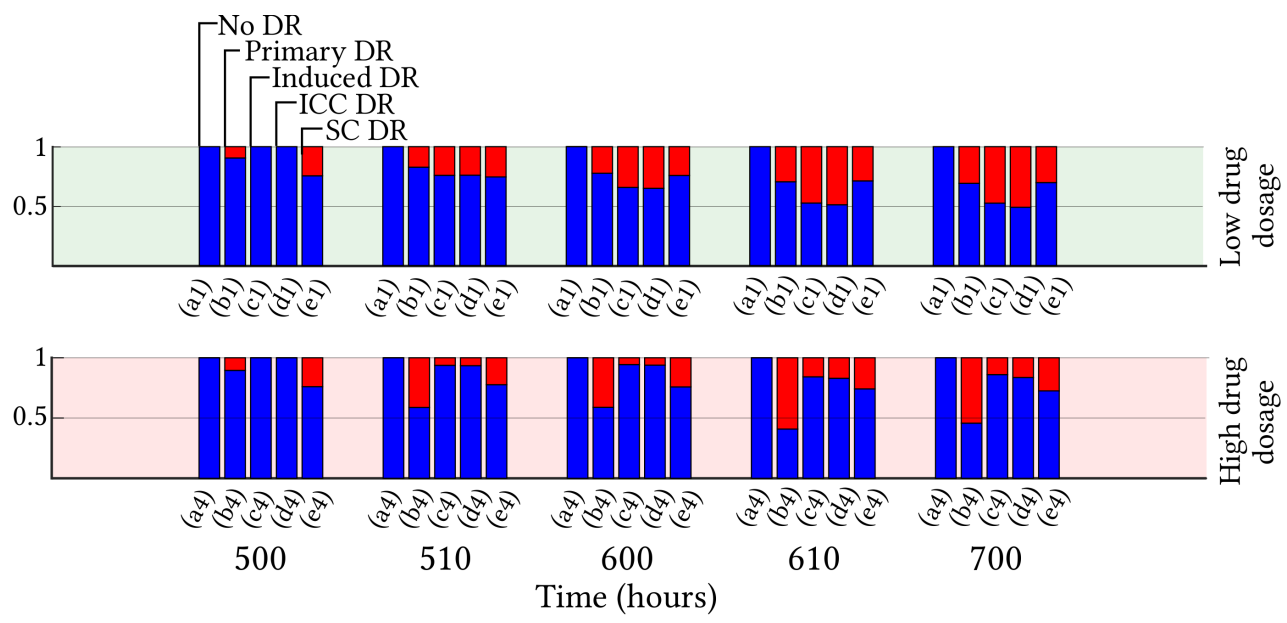

Figure 3.13: Diagram of the ratio between the drug sensitive subpopulation (blue) and the drug resistant sub-population. Various types of drug resistance are incorporated namely (a) No DR, (b) Primary DR, (c) Induced DR, (d) ICC DR, (e) SC DR. Results are shown at times $500 \mathrm{~h}$ (immediately before the first drug dose), $510 \mathrm{~h}, 600 \mathrm{~h}$ (immediately before the second drug dose), $610 \mathrm{~h}$ and $700 \mathrm{~h}$ (end of simulation) for low drug dosages, (1) 1C, and high drug dosage, (4) $8 \mathrm{C}$ according to the labelling in Table 3.5 . Each diagram shows the average value based on 100 simulations. 


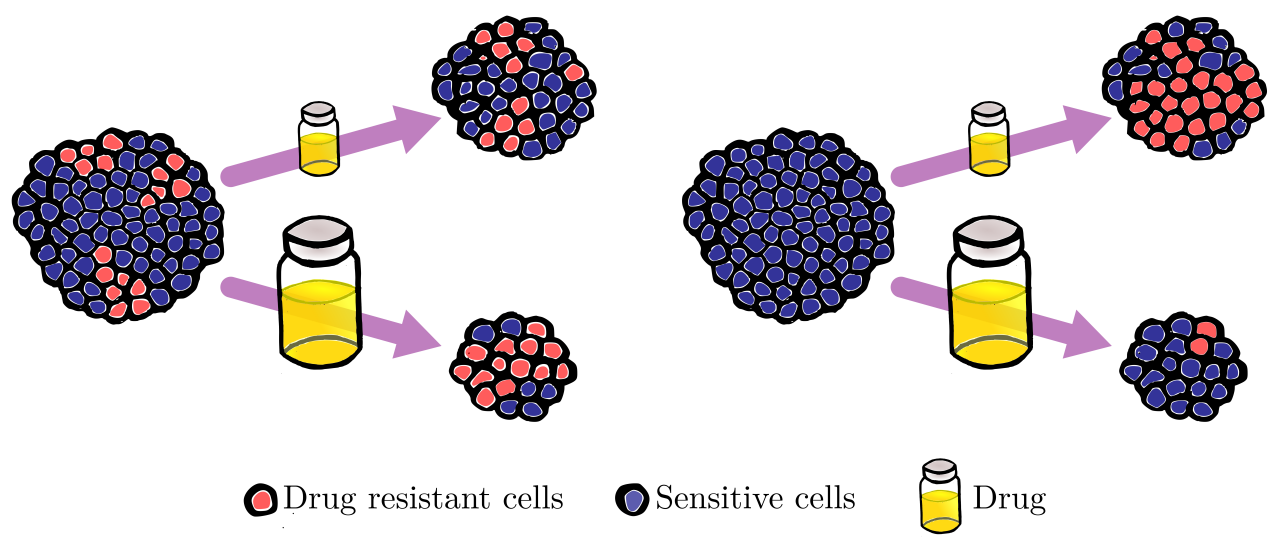

Figure 3.14: Tumours exposed to chemotherapeutic drugs given in low (top) or high (bottom) dosages, the latter dosage option kills more cancer cells. One of the tumours (left) expresses drug resistance pre chemotherapy, the other one (right) does not. Generally in our in silico experiment, drug resistance that occurs independently of the drug, and thus may precede chemotherapy, is amplified by high drug dosages (left). Conversely, drug resistance that is induced by drug presence is accelerated by low to moderate drug dosages (right).

Nietzschean philosophy, chemotherapy that does not kill a tumour may indeed make it stronger. Generally we found that drug resistance presenting independently of the drug, which thus may precede chemotherapy, is amplified by high drug dosages. However, drug resistance that is induced by drug presence is accelerated by low to moderate drug dosages. These findings are pictorially demonstrated in Figure 3.14 . 
3. DRUG RESISTANCE 


\section{Chapter 4}

\section{Hypoxia-Activated Prodrugs}

Hypoxia-Activated Prodrugs are bioreductive prodrugs convert to cytotoxic agents upon reaching hypoxic (tumour) regions. Thus they, theoretically, act as Trojan horses, being essentially harmless until warheads are released in tumours. In this chapter we investigate treatments combining hypoxia-activated prodrugs and radiotherapy.

\subsection{Chapter Summary}

Hypoxia-activated prodrugs (HAPs) present a conceptually elegant approach to not only overcome, but better yet, exploit intra-tumoural hypoxia. Despite being successful in vitro and in vivo, HAPs are yet to achieve successful results in clinical settings. It has been hypothesised that this lack of clinical success can, in part, be explained by the insufficiently stringent clinical screening selection of determining which tumours are suitable for HAP treatments [162].

We here demonstrate that both the intra-tumoural oxygen landscape and treatment scheduling of HAP-radiation combination therapies influence treatment responses in silico. Our in silico framework is based on an on-lattice, hybrid, multiscale cellular automaton spanning three spatial dimensions. The mathematical model is parameterised by multicellular tumour spheroid (MCTS) data from a previous study by Voissiere et al. [3]. 


\section{HYPOXIA-ACTIVATED PRODRUGS}

\subsection{Introduction}

Oxygen concentrations vary across solid tumours and, although tumours present with high diversity across patients [163], hypoxic regions are prevalent tumour features [66, 88, 164-170] commonly provoked by inadequate oxygen supply and high tumour growth rates [85]. Hypoxia significantly impacts tumour dynamics, treatment responses and, by extension, clinical outcomes [165, 168, 171]. Hypoxia may alter cellular expressions of genomes, proteins and epigenetic traits [85], and such hypoxia-induced alerations may cause hypoxic cancer cells to be more resistant to apoptosis [172]. Hypoxia may also alter the metabolism of cells [172] and promote angiogenesis by activating associated genes [173]. Thus hypoxia both protects and progresses solid tumours [171, 172]. Accordingly, severe tumour hypoxia is associated with tumours that are difficult to treat and, by extension, poor prognoses for patients [85, 166]. It is well established that hypoxic regions in solid tumours express reduced sensitivity to radiotherapy and a plethora of chemotherapeutic drugs [65, 85, 165-168, 170, 172-175]. Hypoxic cancer cells in a solid tumour are naturally located far away from active oxygen sources, i.e. blood vessels [166], and therefore drug molecules that are of large size or tightly bound to cell components may not reach hypoxic tumour cells at all [173]. Moreover, genes associated with chemo-resistance may be upregulated by hypoxia [162]. Hypoxia is also regarded to be one of the main factors contributing to radiotherapy failure [173], as is previously explained in Section 2.6.2. IR-induced DNA damage, especially in the form of double strand breaks, is more easily self-repaired by the cell under hypoxic conditions [79].

Due to their severe impact on conventional anticancer therapies, such as chemotherapy and radiotherapy, hypoxic cancer cells, and their central mediators [85, have for the last decades been considered to be important treatment-targets [163, 173]. Multiple ways to handle tumour hypoxia have been explored. One approach to combat intratumoural hypoxia is to increase the tumour oxygenation as part of a neoadjuvant treatment [162]. A second approach to overcome hypoxia is to selectively target hypoxic cancer cells only for treatment-sensitising or eradication [88. A third and conceptually elegant approach to not only overcome, but better yet, exploit intratumoural hypoxia is realised by hypoxia-activated prodrugs (HAPs) [173]. HAPs are bioreductive prodrugs that reduce, and thus convert, into cytotoxic agents upon reaching hypoxic (tumour) 
regions [65, 172]. Theoretically, they act as Trojan horses, being essentially harmless until they are converted into warheads in target regions, i.e. hypoxic (tumour) cells. The tumour-targeting ability of HAPs is based on the premise that oxygen concentrations in hypoxic tumour regions reach exceptionally low levels, and that such low oxygen levels are not found elsewhere in the surrounding body tissue [172]. Indeed physoxia, that is the term used to describe oxygen levels found in normal tissue, ranges between 10 and $80 \mathrm{mmHg}$, and a cancer cell is commonly classified as hypoxic if it has a partial pressure of oxygen $\left(\mathrm{pO}_{2}\right)$ value of $10 \mathrm{mmHg}$ or less [66]. Solid tumours commonly display regions that are even more hypoxic, where $\mathrm{pO}_{2}$ values may drop below $5 \mathrm{mmHg}$ [66]. Consequently, HAPs theoretically constitute a means to effectively target hypoxic tumour cells. This also means that toxic drug effects can be localised to tumours, and that the remaining host system can in great part be spared from harmful toxicity causing unwanted side effects.

HAPs transform into activated drugs (AHAPs) via reductive metabolism [164, 173] in sufficiently hypoxic environments, and the AHAPs can achieve cytotoxic effects in cells [103]. Freely available redmolecular oxygen inhibits this bioreduction, and thus HAPs remain (for the most part) intact in well-oxygenated environments [172]. Once activated, AHAPs may diffuse into local surroundings. Thus, via bystander effects, AHAPs may infer damage to cells in which the HAP-to-AHAP bioreduction did not occur, however a few recent studies dispute the impact of these bystander effects on the overall treatment outcome [176]. In the mathematical model described in this study, the dispersion of HAPs and AHAPs obey mechanistic diffusion equations, and the reach of AHAPs can easily be modified by altering coefficients in the AHAP diffusion equation. Thus the influence of bystander effects on the treatment outcome is allowed to range from negligable to highly influential.

Multiple HAPs have been evaluated for their clinical potential, both as monotherapies and as part of combination therapies [85, 167. Class I HAPs are activated in moderately hypoxic environments whilst Class II HAPs require more severe hypoxia to undergo the HAP to AHAP bioreduction [177]. One such Class II HAP is evofosfamide, or TH-302, which has been tested in clinical Phase I-III trials [85, 162]. TH-302 bioreduces to its activated form, bromo-isophosphoramide mustard (Br-IPM), in hy- 


\section{HYPOXIA-ACTIVATED PRODRUGS}

poxic tumour regions, and Br-IPM is a DNA-crosslinking agent [176]. Multiple in vitro and in vivo studies have validated this drug's preclincal success and, by extension, its clinical feasibility [103, 165, 166, 168, 169, 171, 175, 178,182. Multimodality treatment strategies combining HAPs, particularly Class II HAPs, with ionising radiation (IR) may be particularly promising [167, 168, 181] as the two therapies conceptually complement each other: HAPs target hypoxic tumour regions whilst radiotherapy is most effective against well oxygenated tumour regions. Thus, in principal, HAP-IR combination treatments have the ability to produce multifaceted attacks on tumours.

Despite HAPs being conceptually promising and successful in laboratories, this success has not yet been mirrored in clinical trials [85, 162, 163. It is hypothesised that this unsuccessful Bench-to-Bedside translation is partly due to an insufficiently stringent clinical screening practice of selecting tumours that are suitable for HAP treatments [162]. It is likely that some of the tumours enrolled in clinical trials have been insufficiently hypoxic to benefit from treatment plans involving HAPs [163]. To investigate this hypothesis, we here propose a mathematical modelling angle to simulate how spatio-temporal tumour features may impact HAP efficacy and how scheduling influences the outcome of multimodality HAP-IR treatments.

Today, mathematical modelling constitutes an indispensable complement to traditional cancer research. Models provide an opportunity to study biological phenomena in silico that may not be empirically observable and, moreover, in silico experiments are fast and cheap to run, easy to reproduce and not directly associated with any ethical concerns. Previous mathematical studies have already contributed to the overall understanding of HAPs, quantified key mechanisms associated to them and illustrated their clinical feasibility. Foehrenbacher et al. [183 have deployed a Green's function method, in customised form, and pharmacokinetic/pharmacodynamic (PK/PD) modelling to quantify anticancer bystander effects elicited by the HAP PR-104 in a simulated, threedimensional tumour comprising a microvascular network. Another concurrent article used similar mathematical concepts to compare Class I HAPs to Class II HAPs and, furthermore, to determine optimal properties for Class II HAPs [177]. Lindsay et al. [184 developed a stochastic model to study monotherapies and combination therapies involving HAPs, specifically TH-302, and erlotinib. Amongst other findings, they 
concluded that a combination therapy of the two drugs impedes the uprising of drug resistance. Since HAPs bioreduce to activated form under hypoxic conditions it follows that AHAP activity increases with intratumoural hypoxia. Accordingly, a previous study by Wojtkowiak et al. [185. conceptually validated the strategy of amplifying TH-302 activity by deliberately exacerbating intratumoural hypoxia using exogenous pyruvate. Their study combined mathematical modelling with metabolic profiling and EPR (electron paramagnetic resonance) imaging. HAP dynamics were modelled using reaction-diffusion/convection equations coupled with fluid-structure interactions. In line with these previous mathematical studies, the aim of this in silico study is to contribute HAP-related insights gained by mathematical modelling, according to a Blackboard-toBedside [186] approach.

In clinical settings, the intratumoural oxygenation status can be assessed in multiple ways. By inserting oxygen electrodes into tumours, $p_{\mathrm{O}_{2}}$ values can directly be measured, but this measuring technique is invasive and does not distinguish between hypoxic and necrotic tumour regions [162]. Alternatively, less invasive imaging techniques, such as positron emission (PET-scans) and oxygen-enhanced magnetic resonance (MRIs), can be used to evaluate oxygen levels in tumours [85, 162]. Consequently, it is indeed feasible to invoke stricter selection regimes when deciding whether or not to pair tumours with HAP treatments in clinical trials [162]. A recent publication, by Spielberg et al. [162, claims that the (lack of) clinical progress with HAP-treatments can, in great part, be attributed to the omission of hypoxia-based patient selection in phase III trials. In this study, we demonstrate that the efficacy of HAP monotherapies and HAP-IR combination therapies in silico is, indeed, highly dependent on tumour-specific oxygen features.

\subsection{Model}

The on-lattice, hybrid, multiscale cellular automaton (CA) presented in Chapter 2 is used in this chapter to model solid tumours subjected to HAP and IR monotherapies, as well as HAP-IR combination therapies. Tumour growth and HAP responses are parameterised by published data from an in vitro study performed by Voissiere et al. [3, in which MCTSs where grown and exposed to HAPs. Specifically, we use their data for human chondrosarcoma HEMC-SS cells exposed to the hypoxia activated prodrug TH- 


\section{HYPOXIA-ACTIVATED PRODRUGS}

302. Our mathematical model is thereafter extended to simulate in vivo drug dynamics in order to investigate scheduling aspects of HAP-IR combination therapies. The parameters used in this chapter can be modified in order to simulate specific cell-lines and drugs, and model rules can be altered in order to simulate both in vitro and in vivo tumours. Thus, with the availability of appropriate data, various tumour scenarios and treatment schedules and doses can be investigated in silico. Hence, the mathematical model presented here constitutes a valuable and versatile complement to both in vitro and in vivo experiments. The model used in this study is an extension of a previous, well-established model presented by Powathil et al. [6]. All parameters used in the model are motivated from experiments and literature, as described throughout this section, and are summarised in Section 4.3.7, Table 4.1.

\subsubsection{Mathematical Framework}

The CA used in this model allows for spatio-temporal dynamics and intratumoural heterogeneity including variations in cell-cycle progression, oxygen levels, drug concentrations and treatment responses amongst cancer cells [6, 186, 187]. The model is multiscale and integrates both intracellular and extracellular regulations. In vitro experiments have demonstrated that MCTSs are more HAP-sensitive than are monolayers. This increase in sensitivity has been attributed to the microenvironment correlated to multilayer cultures [175]. Aspiring to achieve an in silico model that is as clinically relevant as possible, we here let the CA lattice extend in three spatial dimensions. The lattice is specifically a square lattice containing $100^{3}$ lattice points, simulating a physical environment of $(2 \mathrm{~mm})^{3}$. Thus each voxel in the lattice spans a volume of $(20 \mu \mathrm{m})^{3}$ and each lattice point may be occupied by either one cancer cell or extracellular matrix only. These dimensions agree with previous mathematical studies [6], and cell population density in the MCTSs that are used to calibrate the model [3]. The time step used to model the temporal progression of the CA is $\Delta t=10^{-3}$ hours, by appropriate non-dimensionalisation of oxygen dynamics [6].

\subsubsection{Cell Cycle Progression}

On an intracellular scale, sub-cellular mechanisms are modelled individually for each cell in order to allow for variations amongst cancer cells. Cell-cycle progression is one such intracellular process, it is governed by an intrinsic cell-cycle clock attributed to each 


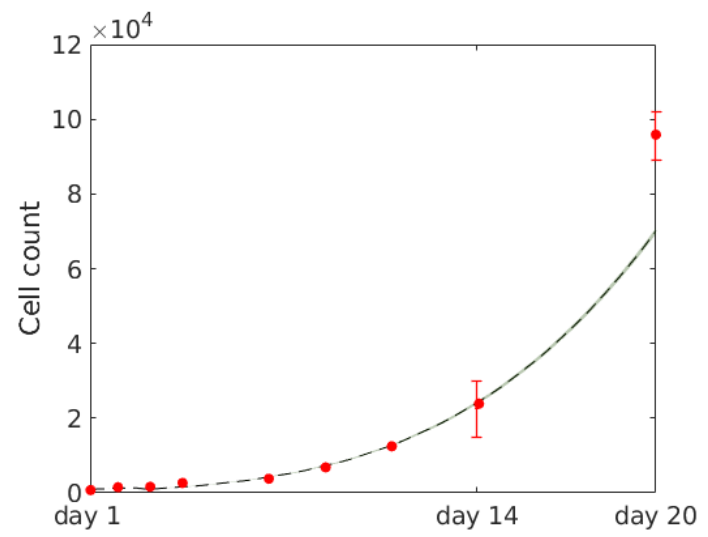

Figure 4.1: Cell count over time for tumour spheroids. The in silico data is based on 10 simulations runs, the mean (black line) shows the average value and the gray ribbon shows standard deviation. In vitro data (red error bars) are extracted from plots produced by Voissiere et al. [3] using a Java program [4].

individual cell. In order to account for cell-cycle asynchronicity amongst cells, each cell $i$ is assigned an individual, stochastic doubling-time $\tau_{i}$ which corresponds to the time it takes for a cell to complete one cell-cycle, and double by producing a daughter cell, in well-oxygenated conditions. $\tau_{i}$ is picked from a normal distribution with a mean value $\mu$ and a standard deviation $\sigma$, which are picked to match cell population growth-rates reported from Voissiere et al. [3]. as demonstrated in Figure 4.1.

Each cell in the model follows a cell-cycle typical to that of eukaryotic cells. In particular, a cell is defined to be in the gap 1 (G1), synthesis (S), gap 2 (G2) or mitoses (M) phase of the cell-cycle. As sensitivity to radiotherapy is cell-cycle dependent [79], it is important to track cell-cycle phase progression in the model. Each cell that is placed on the lattice commences its first cell-cycle in the G1 phase. Under well-oxygenated conditions, the fraction of time spent in each of the four distinct cell-cycle phases are $\Theta_{G 1}, \Theta_{S}, \Theta_{G 2}$ and $\Theta_{M}$ for the cell-cycle phases G1, S, G2, M respectively, where the $\Theta$-fractions sum up to, as described in Chapter 2.2.2, and the four theta values, listed in Table 4.1, are picked from literature in order to match typical lengths of cell-cycle phases for human cells with a doubling time of roughly 24 hours [63]. Thus, the time spent in each of the four distinct cell-cycles, for a well-oxygenated cell $i$ with a cell-cycle length $\tau_{i}$, is $\Theta_{G 1} \tau_{i}, \Theta_{S} \tau_{i}, \Theta_{G 2} \tau_{i}$ and $\Theta_{M} \tau_{i}$ for the cell-cycle phases G1, S, G2 and M respectively. However, low cellular oxygen levels have been shown to delay cell-cycle 


\section{HYPOXIA-ACTIVATED PRODRUGS}

progression by inducing arrest in particularly the G1 phase of the cell cycle [1] and thus, in accordance with the cell cycle clock model described in Chapter 2.2.2, the G1 phase is elongated under hypoxic circumstances according to Equation 2.3 and Figure 2.2

\subsubsection{Tumour Growth}

In the model, a tumour is grown from one seeding cancer cell which divides and gives rise to a heterogeneous MCTS. Once a viable, i.e. undamaged, cell has completed the mitoses (M) phase of the cell-cycle, a secondary cell, namely a daughter cell, is produced and placed in the neighbourhood its mother cell. This cell-division occurs provided that free space is available on the lattice in the neighbourhood of the mother cell. If this is not the case, no daughter cell is produced and instead the mother cell exits the cell-cycle to enter the quiescent phase, G0. Should neighbourhood space be made available again, as a result of cells getting removed from the lattice due to anticancer treatments, quiescent cells may re-enter the cell-cycle. Each daughter cell is placed on a random lattice point in the neighbourhood of the mother cell, where up to $\nu$ spherical neighbourhoods are regarded. In order to agree with the MCTS data [3] used to calibrate the model, $\nu=3$, as illustrated in Figure 4.2, and thus a daughter cell may be placed up to three neighbourhoods away from its mother cell. To accomplish spherical-like tumour growth the model stochastically alternates between deploying Moore and von Neumann neighbourhoods [6].

\subsubsection{Oxygen Distribution and Hypoxia}

Oxygen is assumed to be readily available in the extracellular space and, accordingly, extracellular lattice points are oxygen source points. On the other hand, viable (i.e. non-damaged) cells are modelled as oxygen sinks as they consume oxygen in order to function. As is previously discussed in this thesis, the distribution of oxygen across the lattice is modelled by a mechanistic partial differential equation (PDE), such that

$$
\frac{\partial K(x, t)}{\partial t}=\nabla \cdot\left(D_{K}(x, t) \nabla K(x, t)\right)+r_{K} m(x, t)-\phi_{K} K(x, t) \operatorname{cell}(x, t)
$$

coupled with no-flux boundary conditions. Here $K(x, t)$ denotes the oxygen level in lattice point $x$ at time $t$. $D_{K}(x, t)$ is the diffusion coefficient, which is higher in lattice 

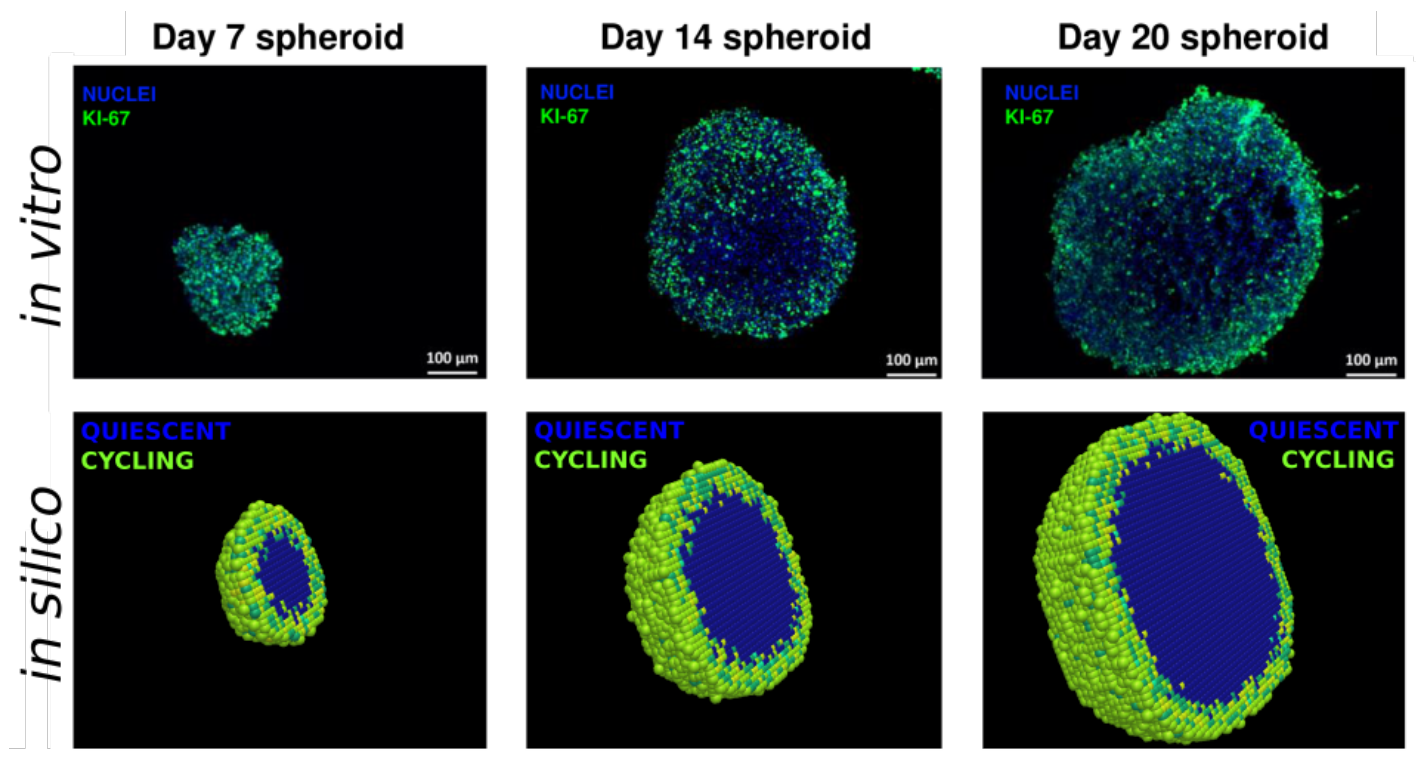

Figure 4.2: Top: Images from in vitro experiments performed by Voissiere et al. [3, in which cell nuclei are stained blue and furthermore proliferative cells are stained green by the proliferation marker Ki-67. Bottom: Images from in silico experiments performed in this study, where proliferative (cycling) cells are coloured green and quiescent cells are coloured blue. 


\section{HYPOXIA-ACTIVATED PRODRUGS}

points occupied by cells compared to unoccupied lattice points, so that oxygen diffuses slower over cancer cells than in extracellular material in the model [6]. The binary function $\operatorname{cell}(x, t)$ is equal to one if the lattice point is occupied by a cancer cell, and zero otherwise. Similarly, the binary function $m(x, t)$ is one if the lattice point is outside the tumour, and zero otherwise. The oxygen production rate is denoted by $r_{K}$ and the cellular oxygen consumption rate is $\phi_{K}$. In this study, the diffusion coefficient for oxygen is gathered from literature but the production and consumption rates are calibrated in silico to match in vitro data from Voissiere et al. [3], specifically to achieve appropriate oxygen gradients. Note that the no-flux boundary condition causes the total amount of oxygen on the lattice to increase over time. To express oxygenation levels on the lattice in scaled form, a scaled oxygen variable $\hat{K}(x, t)$ is introduced which is obtained by

$$
\hat{K}(x, t)=\frac{K(x, t)}{\max _{n} K(n, t)} \cdot h,
$$

where $\max _{n} K(n, t)$ denotes the maximal $K(x, t)$-value (of all $n$ lattice points) at time $t$ [67]. The scaling-factor, $h$, (with unit $\mathrm{mmHg}$ ), is incorporated in order to calibrate the model to fit the MCTS data, as illustrated in Figure 4.3. A cell is defined to be hypoxic if it has a scaled oxygen value such that $\hat{K}(x, t) \leq 10 \mathrm{mmHg}$ [6]. In the model, the $\hat{K}(x, t)$-value influences G1-arrest (Figure 2.2), radio-sensitivity (Figure 2.6) and HAP-AHAP bioreduction rates (Figure 4.4).

\subsubsection{Hypoxia Activated Prodrugs}

Anticancer prodrugs constitute relatively harmless compounds in their inactivated form with the potential to bioreduce, or transform, into cytotoxic species [103]. Specifically for HAPs, this bioreduction occurs in hypoxic conditions and thus HAPs are able to selectively target hypoxic tumour regions [103]. The oxygen dependent bioreduction is here modelled by the function $f_{H A P \rightarrow A H A P}(\hat{K}(x, t))$, where

$$
f_{H A P \rightarrow A H A P}(x, t)=b \cdot B R F(\hat{K}(x, t)),
$$

where $b$ is a time-scaling factor with and $B R F$ is a bioreduction factor as illustrated in Figure 4.4 and

$$
\operatorname{BRF}(\hat{K}(x, t))=\frac{\left[p O_{2}\right]_{50}}{\left[p O_{2}\right]_{50}+\hat{K}(x, t)} .
$$



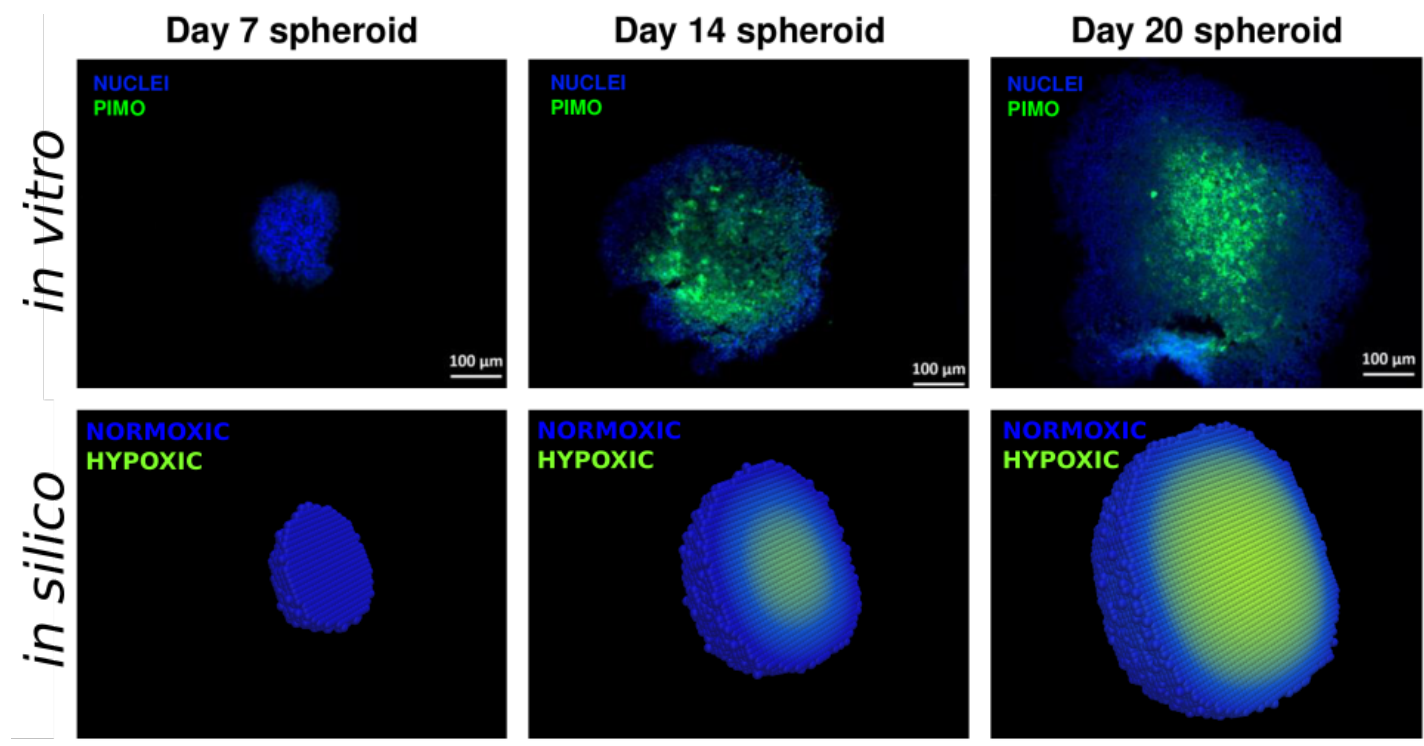

Figure 4.3: Top: Images from in vitro experiments performed by Voissiere et al. [3], in which hypoxic cells are stained green by pimonidazole and normoxic cells are stained blue. Bottom: Images from in silico experiments performed in this study, where hypoxic cells $\left(p_{\mathrm{O}_{2}} \leq 10 \mathrm{mmHg}\right)$ are coloured green and normoxic cells $\left(p_{\mathrm{O}_{2}}>10 \mathrm{mmHg}\right)$ are coloured blue. 


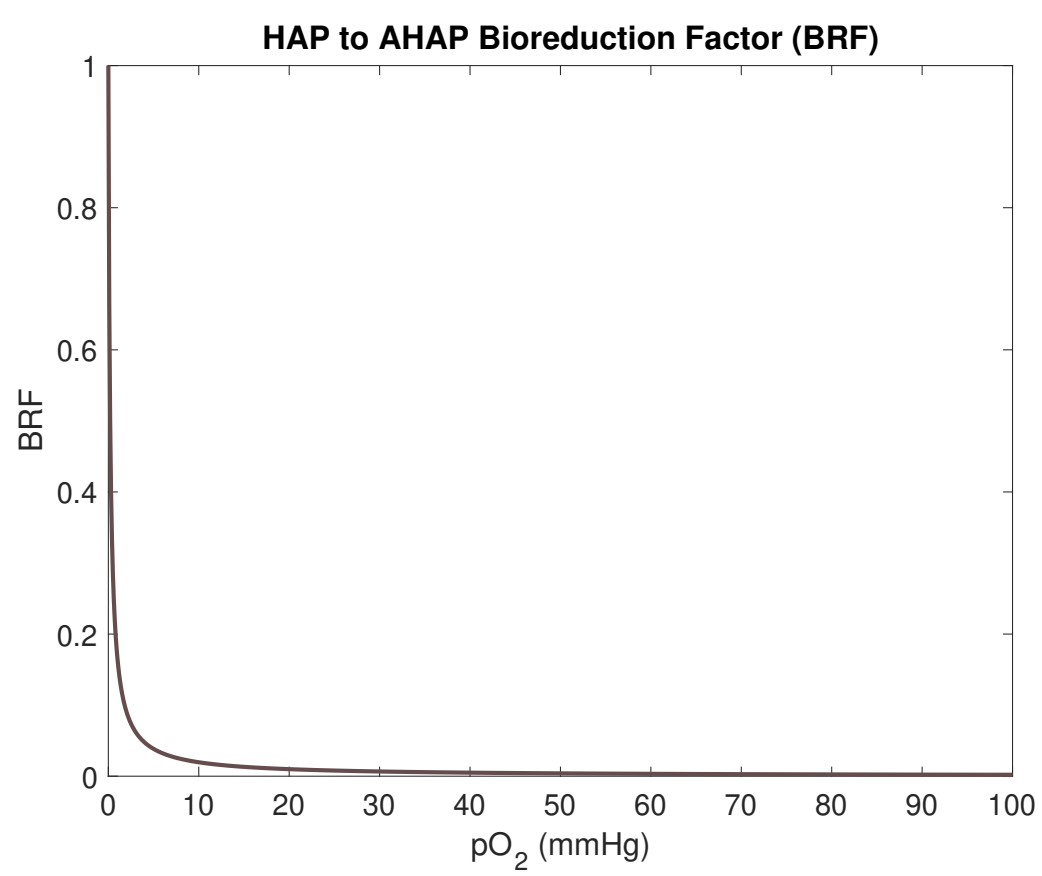

Figure 4.4: The bioreduction factor, $B R F$, expresses the fraction of HAP compound that reduces to AHAP compound within one hour as a function of oxygenation (measured in $\mathrm{mmHg}$ )

Here $\left[\mathrm{pO}_{2}\right]_{50}$ denotes the oxygen value yielding $50 \%$ bioreduction (in one hour), chosen to be $0.2 \mathrm{mmHg}$ as is done in a previous mathematical model by Hong et al. [188]. As illustrated in Figure 4.4 , the BRF value rapidly decreases for $p_{O_{2}}$ values (i.e. $\hat{K}(x, t)$ values) between 0 and $10 \mathrm{mmHg}$.

The mechanistic reaction-diffusion equations governing the distribution of HAPs and AHAPs across the lattice are respectively given by [189]

$$
\begin{aligned}
\frac{\partial[H A P](x, t)}{\partial t}=\nabla \cdot\left(D_{[H A P]}(x, t) \nabla[H A P](x, t)\right)+r_{[H A P]}(x, t) m(x, t) \\
-f_{H A P \rightarrow A H A P}(x, t)[H A P](x, t)-\eta_{[H A P]}[H A P](x, t),
\end{aligned}
$$




$$
\begin{aligned}
\frac{\partial[A H A P](x, t)}{\partial t}=\nabla \cdot( & \left.D_{[A H A P]}(x, t) \nabla[A H A P](x, t)\right) \\
& +f_{H A P \rightarrow A H A P}(x, t)[H A P](x, t)-\eta_{[A H A P]}[A H A P](x, t),
\end{aligned}
$$

where $[H A P](x, t)$ denotes the concentration of HAPs and $[A H A P](x, t)$ denotes the concentration of AHAPs in point $x$ at time $t . D_{[H A P]}(x, t)$ and $D_{[A H A P]}(x, t)$ denote the respective diffusion coefficients, $r_{[H A P]}(x, t)$ denotes the HAP production rate, $\eta_{[H A P]}$ and $\eta_{[A H A P]}$ denote the corresponding decay rates. AHAPs are harmful agents which are here assumed to inflict damage that is cell-cycle non-specific. Consequently, cells that are in any cell-cycle phase (G1, S, G2, M) or in the quiescent phase (G0) are susceptible to AHAP-inflicted damage. A cell in point $x$ at time $t$ is damaged by the cytotoxic AHAPs if $[A H A P](x, t) \geq \Psi$, where $\Psi$ is the lethal AHAP concentration threshold. $\Psi$ and the production coefficient in Equation 4.5 are calibrated in silico to make it so that HAPs and IR yield the same effect (in terms of number of cells killed) for a large tumour (see the Large Tumour in Figure 4.5). When a cell dies, it reduces to a membrane-enclosed cell-corpse which is (in vivo) digested by macrophages [7]. In the model, the time it takes for a cell to receive lethal damage until it is removed from the lattice, to give space to other cells, is denoted $T_{L \rightarrow R}$ ( $\mathrm{L}$ for lethal, $\mathrm{R}$ for removal). Three cases for this time $T_{L \rightarrow R}$ are investigated in this study: $(i)$ the first extreme case in which a dead cell in never removed from the lattice (simulating an in vitro environment), (ii) the other extreme case in which a cell is instantaneously removed from the lattice upon receiving lethal damage, and (iii) a mid-way case in which a cell is removed from the lattice after a time-period corresponding to its doubling time has passed, i.e. $T_{L \rightarrow R, i}=\tau$. Results using the first case are included in the main text, results for cases (ii) and (iii) are provided in Appendix A2 in which we demonstrate that, within the scope of the performed in silico experiments, this choice of $T_{L \rightarrow R}$ value does not affect our qualitative findings.

\subsubsection{Drug Parameters}

In our mathematical model, HAPs are produced on the source points (i.e. extracellular lattice points outside the tumour) and are quickly distributed across the lattice. Drug 


\section{HYPOXIA-ACTIVATED PRODRUGS}

transportation of HAPs from source points to cells is mediated only by the diffusion terms in Equation 4.5 and and similarly AHAP transportation is mediated only by the diffusion term in Equation 4.6. Consequently, the drug diffusion coefficients $D_{[H A P]}$ and $D_{[A H A P]}$ represent all biophysical drug transportation across the lattice in silico. HAPs must possess certain appropriate attributes in order to produce desired effects [175]. Specifically, HAPs should be able to travel relatively long distances without being metabolised, specifically distances longer than that of which oxygen travels, in order to reach hypoxic tumour regions. It has, indeed, been demonstrated in vivo that TH-302 has the ability to reach hypoxic regions, where it is activated [190]. Conversely, AHAPs should travel relatively short distances in order to localise AHAP activity to tumour regions only, and thus to minimise unwanted extratumoural toxicity. The diffusion length of oxygen is reported in literature to be approximately $100 \mu \mathrm{m}$ [6] however, to our knowledge, no diffusion length of neither TH-302 nor Br-IPM has been explicitly reported. However, the diffusion length of the HAP/AHAP pair AQ4N/AQ4 has been shown to be reach roughly 1.5 times that of oxygen (or $150 \mu \mathrm{m}$ ) in xenografts [191. With this motivation, we here approximate the diffusion coefficient of TH-302 to be twice that of oxygen. (According to the relationship $L=\sqrt{D / \Phi}$, where $L$ is the diffusion length scale and $\Phi$ is the compound uptake, the diffusion coefficient of a certain compound, $D$, is proportional to $L^{2}$, neglecting details of compound uptake. Thus here we make the simplified approximation that $L_{[H A P]}(x, t)=\sqrt{2} \cdot D_{K}(x, t)$. ) Similar to previous procedure, the diffusion length of AHAPs is approximated to be half that of oxygen so that $D_{[A H A P]}(x, t)=(1 / 4) \cdot D_{K}(x, t)$. These parameter estimations suffice to conceptually, and qualitatively, describe the nature of HAPs and AHAPs, but can be amended upon the availability of new data. By adjusting the diffusion coefficients $D_{[H A P]}$ and $D_{[A H A P]}$, the influence of bystander effects are allowed to range from negligible to highly influential.

The half-life times of TH-302 and Br-IPM have been reported to be $0.81 \mathrm{~h}$ and $0.70 \mathrm{~h}$ respectively in a clinical trial [170], these values are used to determine the decay rates $\eta_{[H A P]}$ and $\eta_{[A H A P]}$. This half-life time of TH-302 is in accordance with preclinical predictions obtained from allometric scaling [180]. Note that the drug decay coefficients, $\eta_{[H A P]}$ and $\eta_{[A H A P]}$ in Equation 4.5 and Equation 4.6 respectively, simulate all drug clearance from the system, i.e. both metabolic clearance and excretion. 


\subsubsection{Radiotherapy}

Cellular responses to radiotherapy are dependent on oxygenation status [88], cell-cycle phase [89, 90], and cell-line characteristics. Cellular radiotherapy responses are here modelled using Equation 2.23, an appropriate CA adaptation of the widely accepted Linear-Quadratic (LQ) model discussed in Chapter 2.6.3. Equation 2.23 reads,

$$
S(x, t)=e^{-d\left([O M F] \alpha(x, t)+d[O M F]^{2} \beta(x, t)\right)},
$$

where details are provided in 2.6.3. To include cell cycle sensitivity, $\alpha$ and $\beta$ are here cell cycle dependent and the oxygen modification factor (OMF), expressed in Equation 2.24 is incorporated to include oxygen sensitivity. The survival probability of a cell in point $x$ at time $t$, exposed to a radiation dose of 2 Gy, is illustrated in Figure 2.6 in Chapter 2.8, where the survival probability depends on both oxygenation and cell-cycle phase details.

\subsubsection{Parameters}

In this study we attempt to replicate the nature of generic eukaryotic cell-lines, the HAP evofosfamide (TH-302) and its corresponding AHAP, Br-IPM. The parameters, which are listed in Table 4.1, are chosen accordingly but can be adapted to represent other specific cell-lines or drugs upon data becoming readily available.

\subsubsection{Implementation and in silico Framework}

The mathematical model is implemented in an in-house computational framework written in $\mathrm{C}++$. A flowchart of the programming code is available in Appendix A2. The PDEs describing oxygen and drug distribution across the lattice are solved using explicit finite difference methods with no-flux boundary conditions. Maps of cancer cells and the microenvironment are visualised in ParaView [161]. Using this computational framework, various experimental in vitro and in vivo scenarios are formulated and simulated in silico. In order to grow an in silico MCTS, one seeding cancer cell is placed on the lattice and this cell divides and gives rise to a heterogeneous MCTS. Such virtual spheroids are thereafter subjected to various treatment combinations comprising HAPs and IR. Treatment commence when MCTSs consist of, in the order of, 100,000 cancer cells or 'agents' in out agent-based model. Due to the high number of agents, 


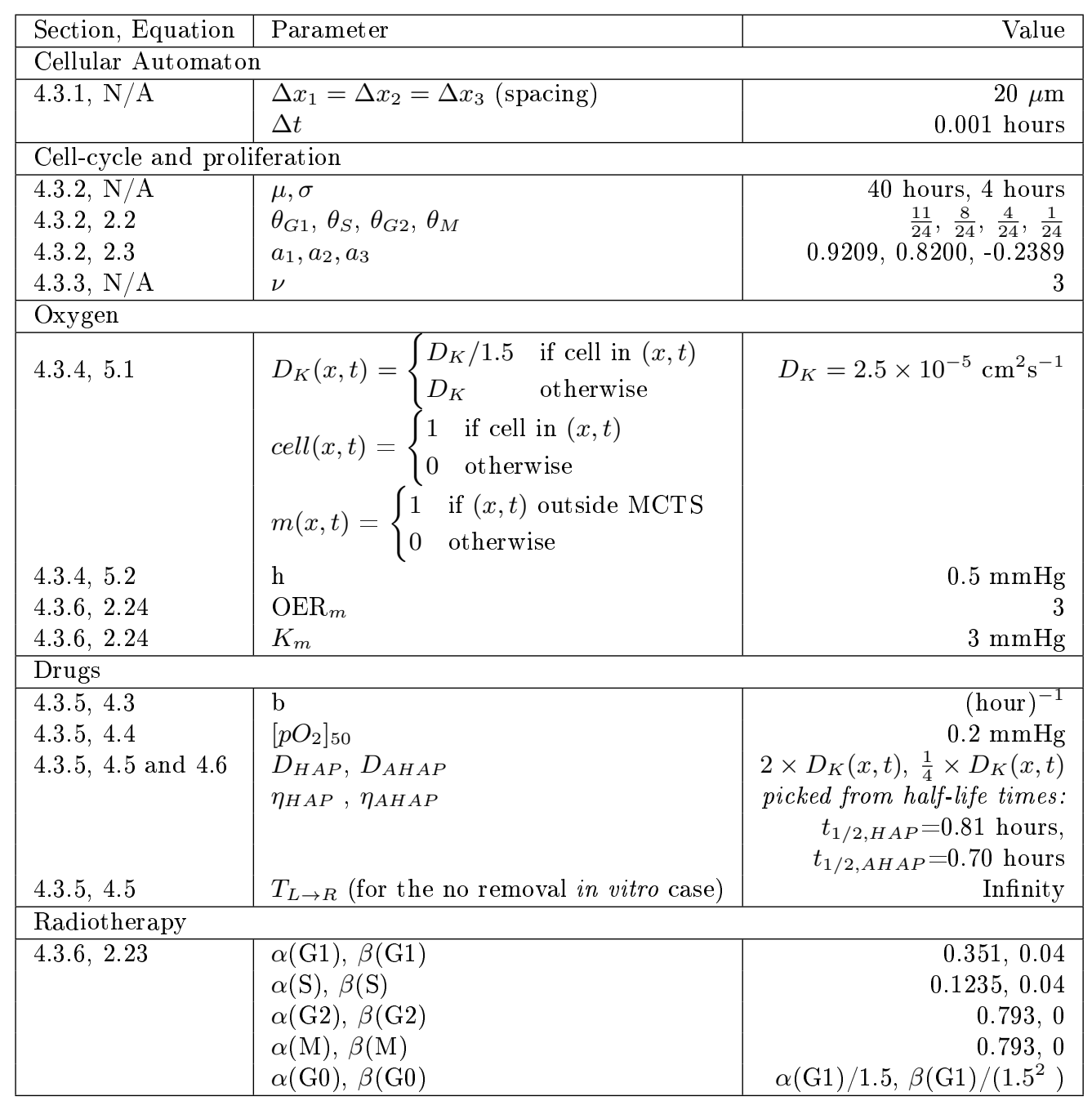

Table 4.1: A summary of model parameters used in the mathematical framework. 
and the fact that the intrinsic model stochasticity only involves a few events during the simulated treatment time (specifically 0-3 cell divisions and potentially one response to radiotherapy) the quantitative results do not differ much between in silico runs. Performing the same in silico experiment 10 times yields a standard deviation that can be regarded as negligible (less than $0.5 \%$ ), and thus we argue that basing our results from means from 10 simulation runs per experiment is enough to mitigate intrinsic model stochasticity to a level that is sufficient for this qualitative study.

\subsection{Results and Discussion}

In Sections 4.4.1 through to 4.4.3, we compare treatment responses in two different in silico tumour spheroids, specifically a 'Large' and more hypoxic MCTS and a 'Small', less hypoxic MCTS. The 'Small' tumour corresponds to the 20 day-old MCTS in Figures 4.2 and 4.3 , that is calibrated by in vitro data from Voissiere et al. [3]. The 'Large' MCTS is extrapolated by letting the 'Small' MCTS grow for yet another 10 days in silico, until it reaches an age of 30 days. The 'Small' and 'Large' MCTSs are illustrated in Figure 4.5

The simulated IR dose is chosen to be $2 \mathrm{~Gy}$, a dose commonly used in clinical settings [79]. To allow for intuitive comparisons between the two different monotherapies, the HAP dose $\left(\right.$ Dose $_{H A P}$ ) is here qualitatively chosen, and calibrated to yield the a similar response as the 2 Gy IR dose (in terms of cell survival) in the 'Large' MCTS. Quantitative doses can be specified and implemented upon the availability of data.

\subsubsection{HAP and IR Monotherapies Attack Tumours in Different Ways}

In this initial in silico experiment, a MCTS is subjected to a monotherapy of either one dose of HAPs or one dose of IR. Our in silico results demonstrate that HAP and IR monotherapies attack the MCTS in different ways. This can be understood by regarding the treatment responses in Figure 4.6 and Figure 4.7. Figure 4.6 shows cell-cycle phase specific survival data, in terms of cell count over time, when the 'Small' or 'Large' MCTS is subjected to a HAP or IR monotherapy. Similarly, Figure 4.7 shows the composition of cells, in terms of their cell-cycle phase, in response to a HAP or IR monotherapy dose. Our results demonstrate that for the 'Small', well-oxygenated MCTS, HAPs have negligible effect on the cell count (see Figure 4.6) and, by extension, on the cell-cycle 


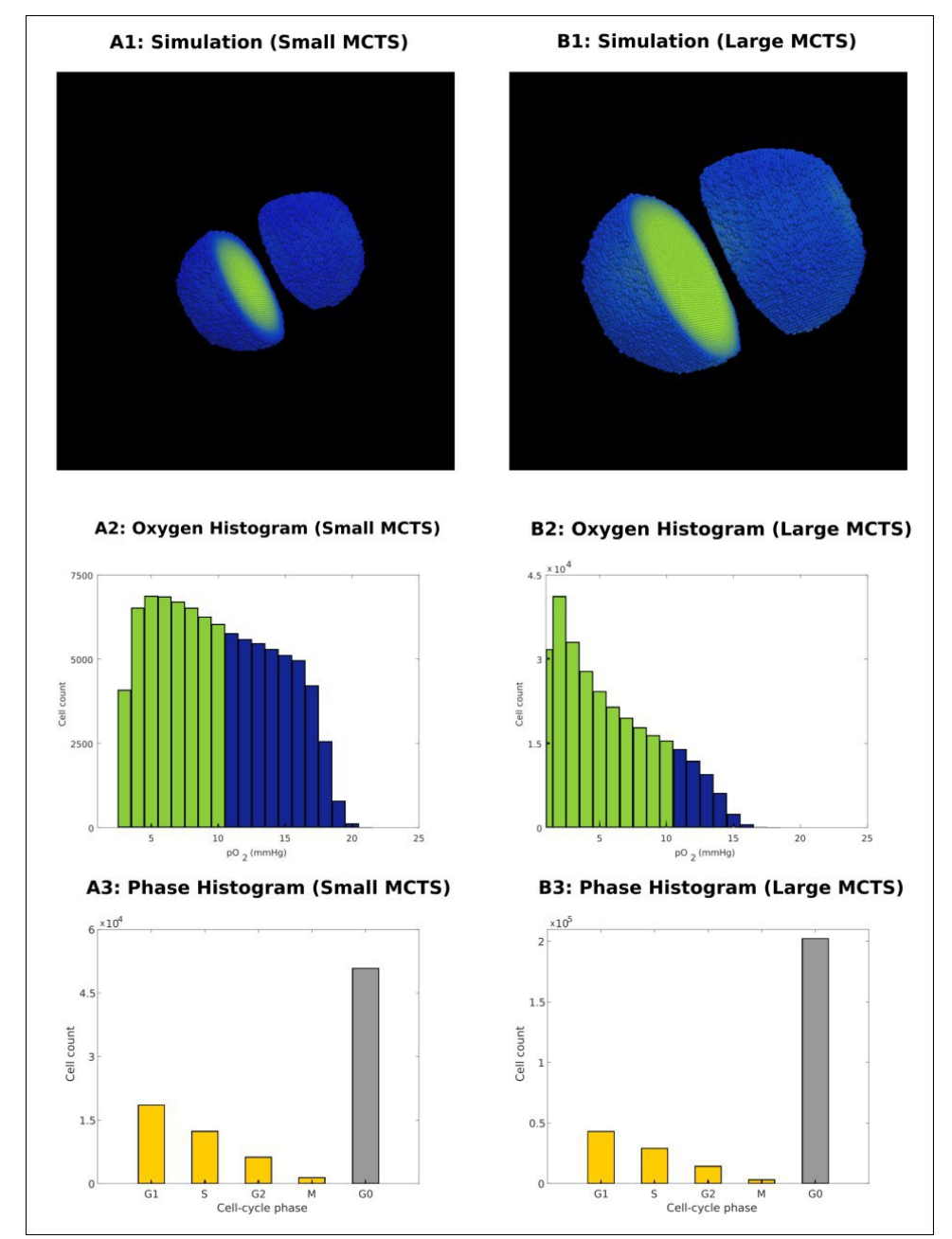

Figure 4.5: The 'Small' (20 day old) MCTS and the 'Large' (30 day old) MCTS are used in Sections 4.4.1, 4.4.2 4.4.3 to allow for comparisons in treatment responses between tumours with different oxygenation levels. Top: Simulation snapshots of the MCTSs at the time point $T_{0}$ when treatments commence (A1: Small MCTS, B1: Large MCTS). Hypoxic cells $\left(p_{\mathrm{O}_{2}} \leq 10 \mathrm{mmHg}\right)$ are green whilst normoxic cells are blue. Middle: Oxygen histograms at time $T_{0}$, in which hypoxic cell counts are shown in green and normoxic cell counts are shown in blue (A2: Small MCTS, B2: Large MCTS). Bottom: Cell-cycle phase histograms at time $T_{0}$ (A3: Small MCTS, B3: Large MCTS).

phase composition (see Figure 4.7). This shows that, by design, HAP treatments have little effect on tumours that are not hypoxic enough to cause significant HAP-to-AHAP bioreduction. For the 'Large' MCTS, however, HAPs successfully eliminate cells, particularly G0 cells (see Figure 4.6). This causes an alteration in the cell-cycle phase composition in favour of proliferative (i.e. non-G0) cells (see Figure 4.7). Our results 
further show that, for both the 'Small' and the 'Large' MCTSs, IR eliminates cells of all cell-cycle phases (see Figure 4.6), but alters the cell-cycle phase composition in favour of G0 cells (see Figure 4.7). These opposing effects on the cell-cycle phase composition achieved by HAPs and IR in the 'Large' MCTS indicate that, for tumours that are hypoxic enough for HAPs to have an effect, HAP-IR combination treatments have the potential of producing multifaceted attacks on tumours.

Since radiation responses are enhanced by the presence of molecular oxygen, we investigated which monotherapy (i.e. HAP or IR) best eliminates hypoxic cells and reoxygenates MCTSs. To demonstrate the overall alteration of oxygenation levels in the MCTSs as a result of the monotherapies, Figure 4.8 provides histograms for cellular oxygenation levels at time $T_{0}$ (the time of therapy administration) and at time $T_{0}+4$ hours. From this figure we can see that for the 'Small' MCTS, HAPs do not alter the overall intra-tumoural oxygenation but IR does. For the 'Large' MCTS, on the other hand, both HAPs and IR alter the overall intra-tumoural oxygenation but only HAPs manage to shift the oxygen histogram away from the most severe levels of hypoxia. This indicates that administering HAPs as a neoadjuvant therapy prior to radiotherapy may enhance the effect of radiotherapy in tumours that are sufficiently hypoxic for HAPs to be effective.

\subsubsection{HAP-IR Treatment Scheduling Impacts HAP Efficacy in Suffi- ciently Hypoxic Tumours}

In order to study the optimal treatment scheduling of HAP-IR combination therapies, simulated MCTSs are here given one dose of HAPs and one dose of IR. Figure 4.9 shows the cell count over time when one dose of HAPs and one dose of IR are administered with various schedules. The results in Figure 4.9 demonstrate that for the 'Small' tumour, scheduling does not impact the overall treatment outcome, as HAPs are not effective. For the 'Large' tumour however, it is more effective to give HAPs before IR than to give IR before HAPs. This indicates that, in tumours that are hypoxic enough for HAPs to be effective, the HAP-IR treatment scheduling impacts the efficacy of the combination treatment. 

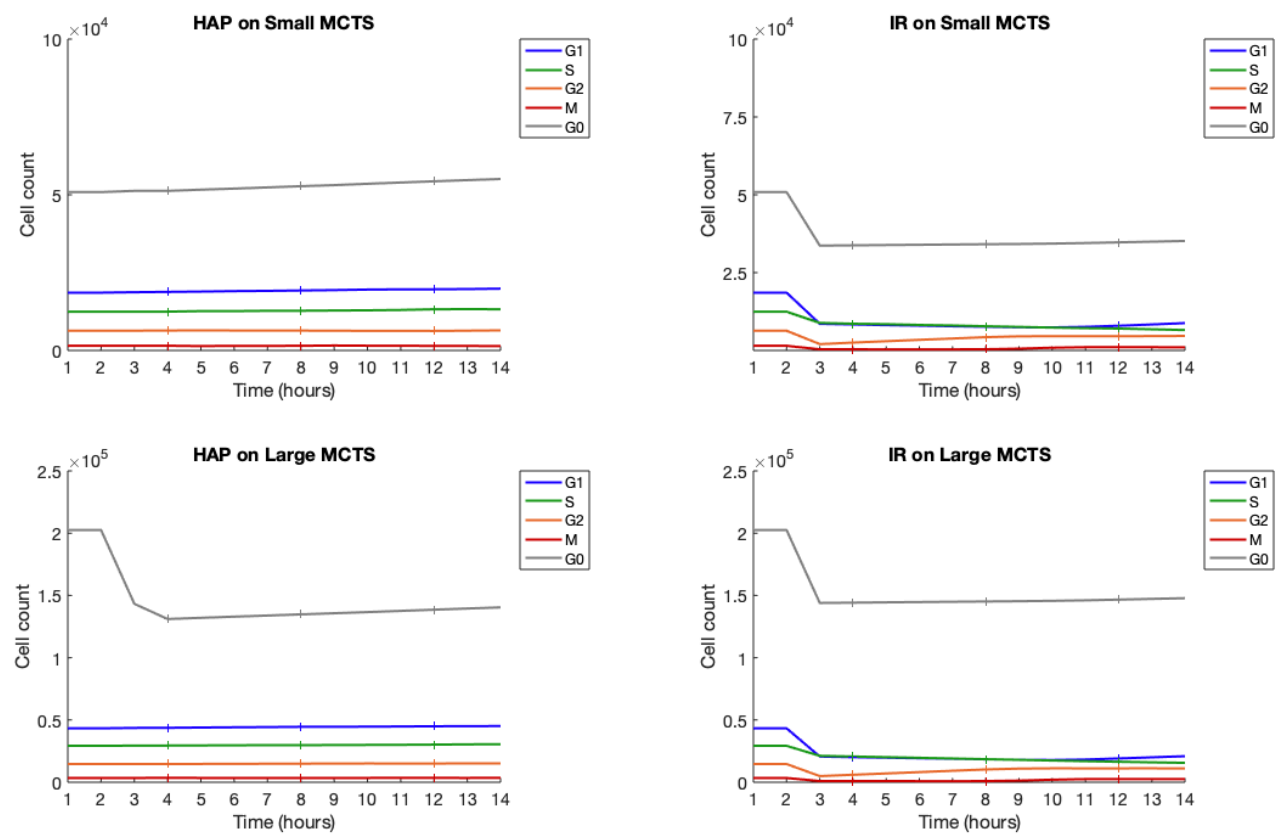

Figure 4.6: Treatment responses for HAPs (left) and IR (right) monotherapies for the 'Small' (top) and 'Large' (bottom) MCTS. The monotherapy is given at 0 hours. Graphs demonstrate cell-cycle specific cell count (i.e. number of viable, undamaged cells) over time. Solid lines show mean values, and ' + ' markers show standard deviations for 10 in silico runs. 

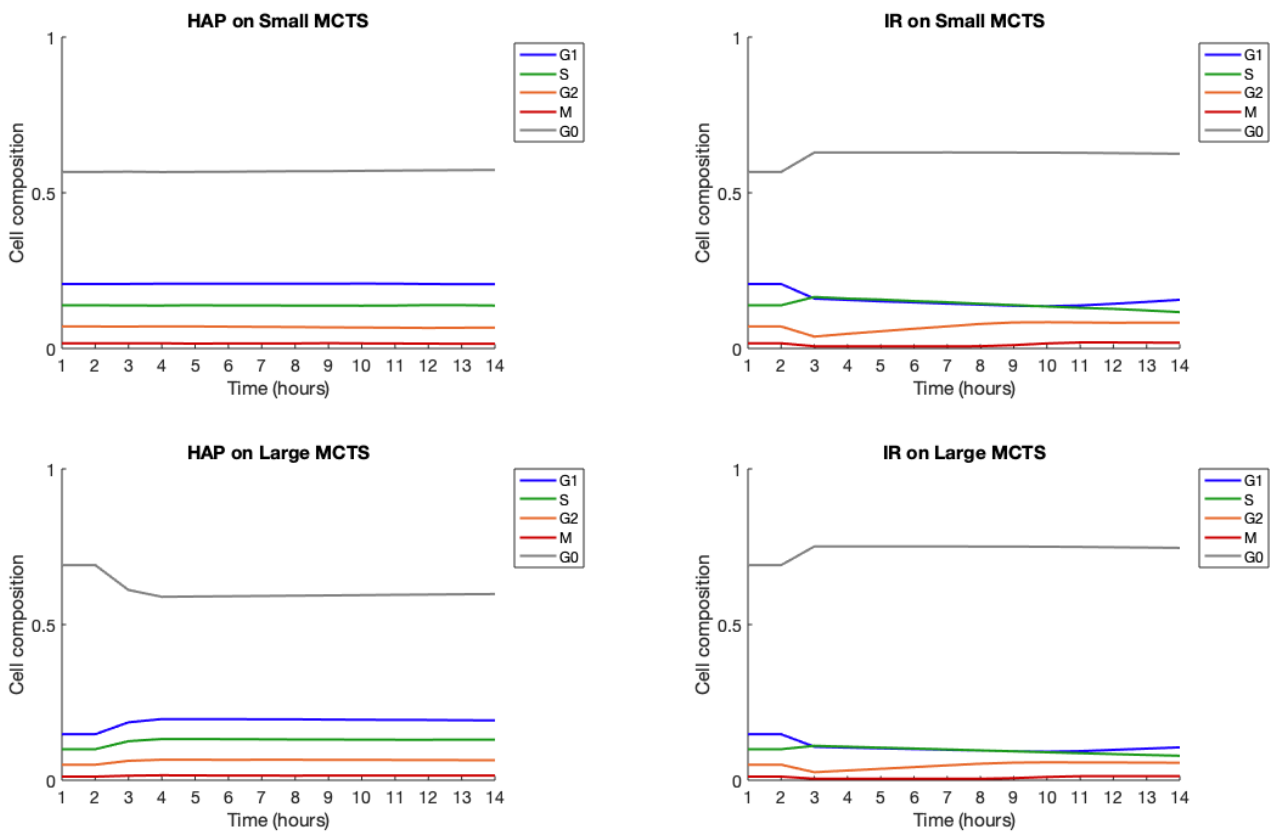

Figure 4.7: Treatment responses for HAPs (left) and IR (right) monotherapies for the 'Small' (top) and 'Large' (bottom) MCTS. The monotherapy is given at 0 hours. Graphs demonstrate cell-cycle specific composition (of viable, undamaged cells) over time. Solid lines show mean values for 10 in silico runs (standard deviations are negligible hence not shown). 

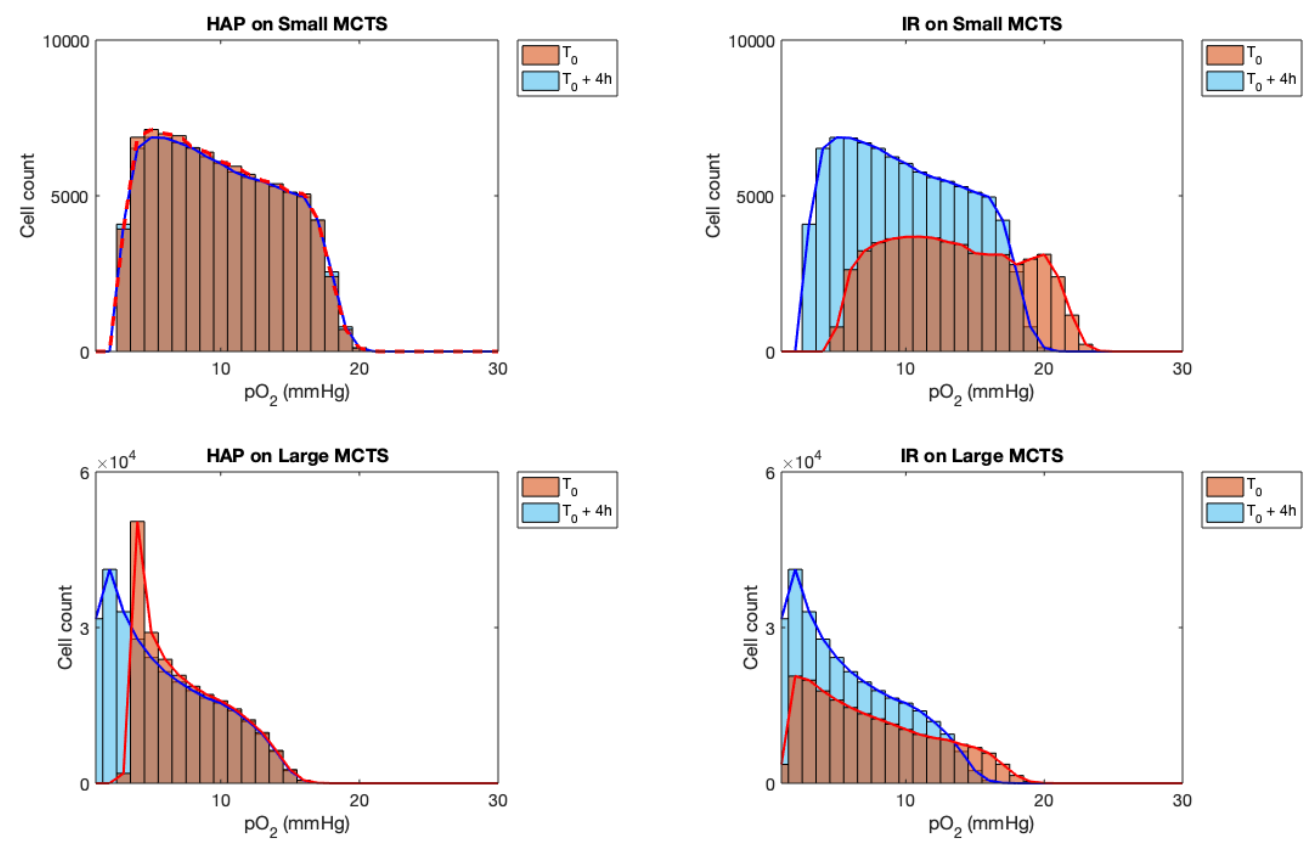

Figure 4.8: Treatment responses for HAPs (left) and IR (right) monotherapies for the 'Small' (top) and 'Large' (bottom) MCTS. Histograms over cellular oxygenation levels at time $T_{0}$ (monotherappy administration time) and 4 hours later are shown. Results are based on mean values from 10 in silico runs. 

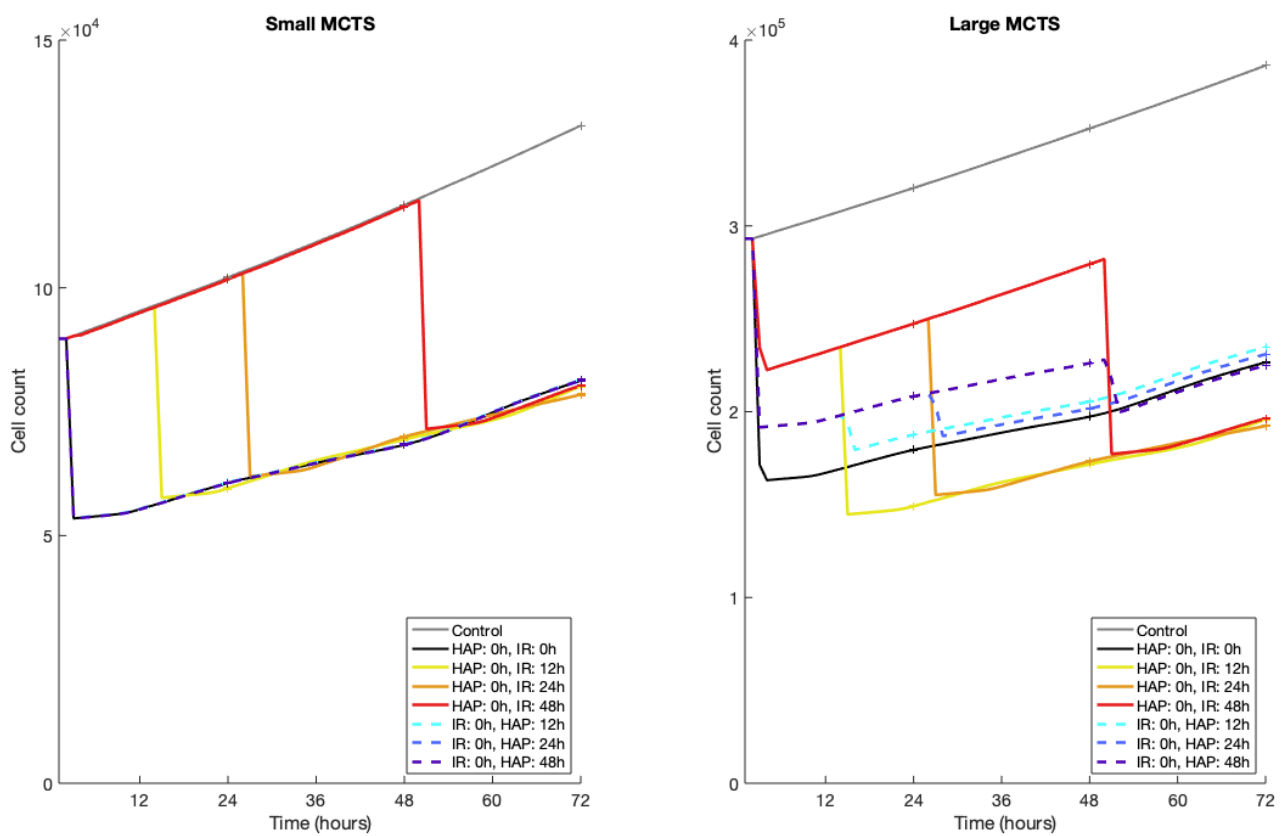

Figure 4.9: Treatment responses (in terms of cell count) for HAP-IR combination therapies in the 'Small' MCTS (left) and the 'Large' MCTS (right). One dose of HAPs and one dose of IR are administered at various schedules. Solid and dashed lines show mean values, and ' + ' markers show standard deviations for 10 in silico runs. 


\section{HYPOXIA-ACTIVATED PRODRUGS}

\subsubsection{HAPs Enhance Radiotherapy Effects in Sufficiently Hypoxic Tumours}

To investigate if and when HAPs enhance the effect of radiotherapy, simulated MCTSs are subjected to either IR monotherapies or HAP-IR combination therapies. In the combination therapy case, HAPs are administered at time $T_{0}$ and IR is administered at time $T_{0}+48$ hours. In the monotherapy case, radiotherapy is administered at time $T_{0}+48$ hours. For a thorough investigation, the oxygen-levels of the 'Large' and 'Small' tumours are further scaled by multiplication with a factor $1,1 / 2$ or $1 / 4$ so that we have 6 different tumours on which to test if neoadjuvant HAPs enhances radiotherapy efficacy. Figure 4.10 shows IR treatment responses in form of survival data (both in terms of number of surviving cells and fraction of surviving cells). From these plots we see that for very hypoxic MCTSs, the administration of neoadjuvant HAPs does increase the effect of radiotherapy. However, for well-oxygenated MCTS, neoadjuvant HAPs do not increase the effect of radiotherapy.

\subsubsection{The Intratumoural Oxygen Landscape Impacts HAP Efficacy}

In Sections 4.4.1, 4.4.2, 4.4.3 we have demonstrated various ways that the intra-tumoural oxygenation level impacts HAP and IR monotherapies and combination therapies. Further, in order to investigate if the spatio-temporal intumoural oxygen landscape impacts HAP efficacy, two MCTSs with different oxygen landscapes are here compared. Omitting details of oxygen dynamics and vessel structure, hypoxic regions are here manually assigned in the MCTSs so that every cancer cell is set to be either severely hypoxic $\left(p_{\mathrm{O}_{2}}=0 \mathrm{mmHg}\right)$ or very well-oxygenated $\left(p_{\mathrm{O}_{2}}=100 \mathrm{mmHg}\right)$. Both MCTSs, named MCTS A and MCTS B, are assigned the same number of severely hypoxic and welloxygenated cancer cells at the time-point when treatment commences. In MCTS A, the hypoxic region is made up of one concentric sphere in the core of the MCTS, whilst in MCTS B, the hypoxic regions consist of multiple spheres, evenly spread out across the MCTS. MCTS A and MCTS B are illustrated in Figure 4.11. The severely hypoxic cancer cells are here called activator cells, as the prodrug bioreduction (or activation) is maximal in severly hypoxic environments. The well-oxygenated cells are here referred to as bystander cells, as the bioreduction is minimal in well-oxygenated environments. Thus any lethal AHAP concentration occurring in a bystander cell is a result of HAPto-AHAP bioreduction occurring outside the bystander cells. 


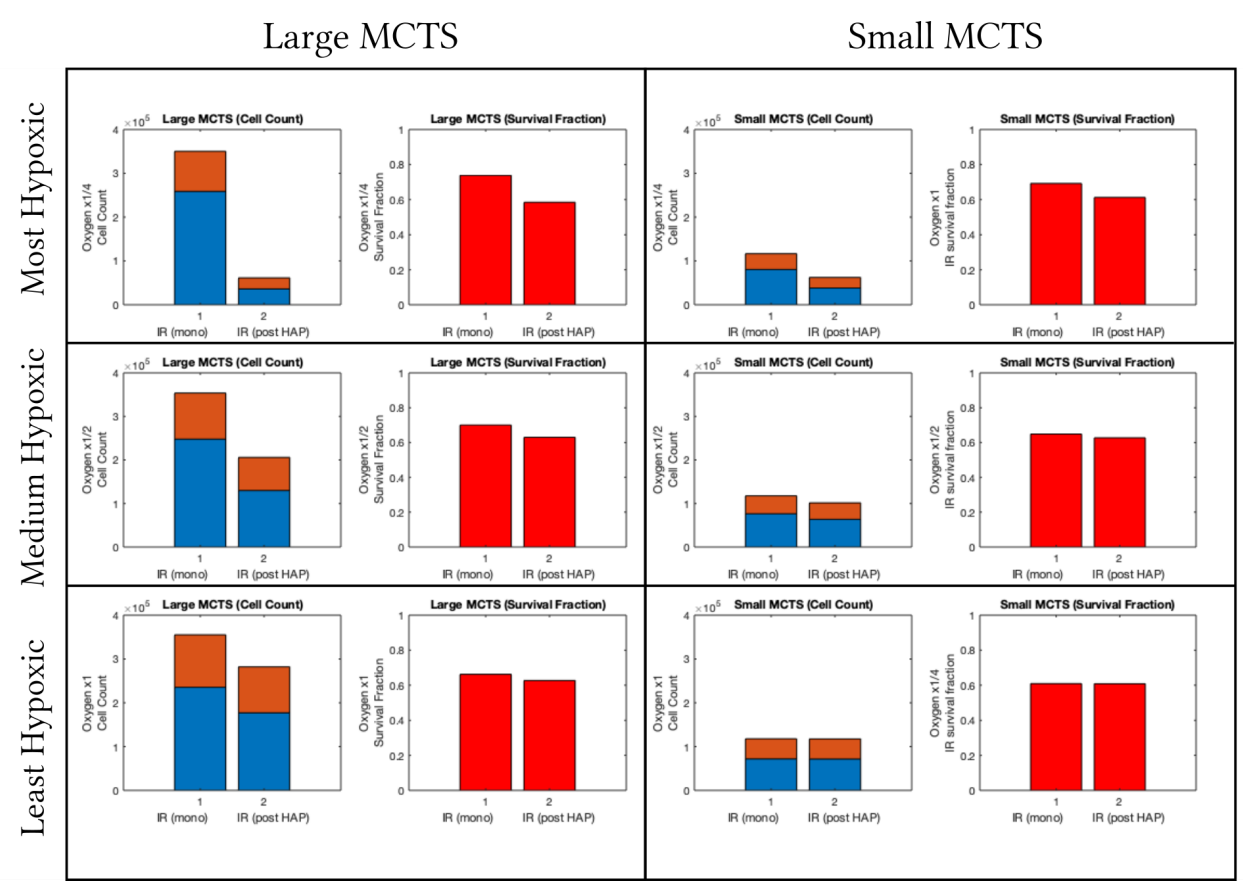

Figure 4.10: Treatment responses of radiotherapy in various MCTSs when either (1) an IR monotherapy dose is administered at $T_{0}+48$ hours or (2) IR is given at $T_{0}+48$ hours following a prior HAP dose at time $T_{0}$. Note that only explicit IR responses (not HAP responses) are shown. The oxygen-levels of the 'Large' (left) and 'Small' (right) tumours are scaled by a factor of 1 (least hypoxic), $1 / 2$ or $1 / 4$ (most hypoxic). The value calibrated from in vitro experiments 3 correspond to the scaling with factor 1 . Yellow bars show number of viable cells (instantaneously) before IR administration, blue bars show the number of viable cells (instantaneously) post IR. Red bars show how many cells (as a fraction) survived the IR attack. 
A1: Simulation (MCTS A)

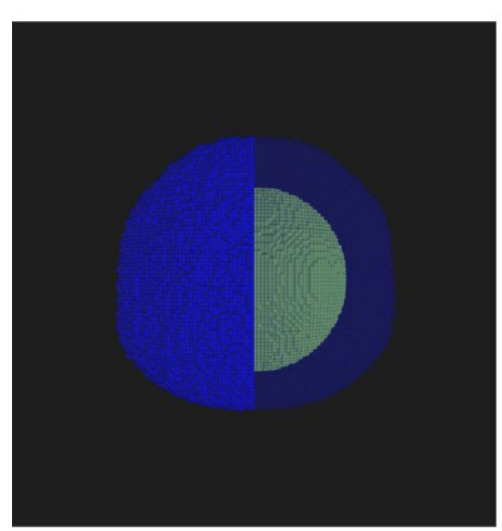

A2: Oxygen Histogram (MCTS A)

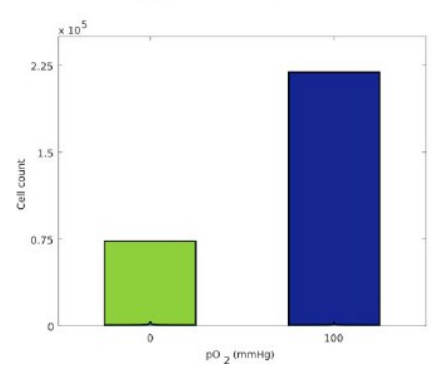

B1: Simulation (MCTS B)

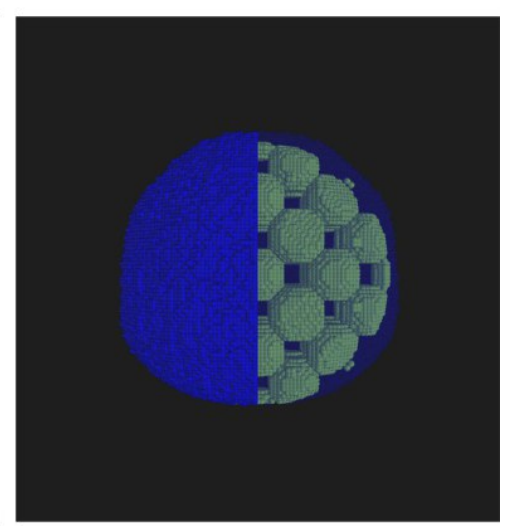

B2: Oxygen Histogram (MCTS B)

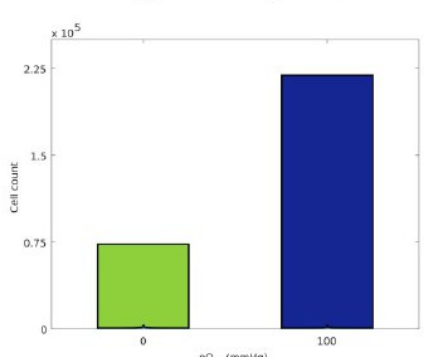

Figure 4.11: Top: MCTS A and B prior to treatment commencing. The MCTSs are visualised in both opaque and transparent formats. Bottom: Oxygen histograms for MCTS $\mathrm{A}$ and $\mathrm{B}$ prior to treatment commencing, hypoxic $\left(p_{\mathrm{O}_{2}} \leq 10 \mathrm{mmHg}\right)$ cell counts are shown in green and normoxic cell counts are shown in blue. 


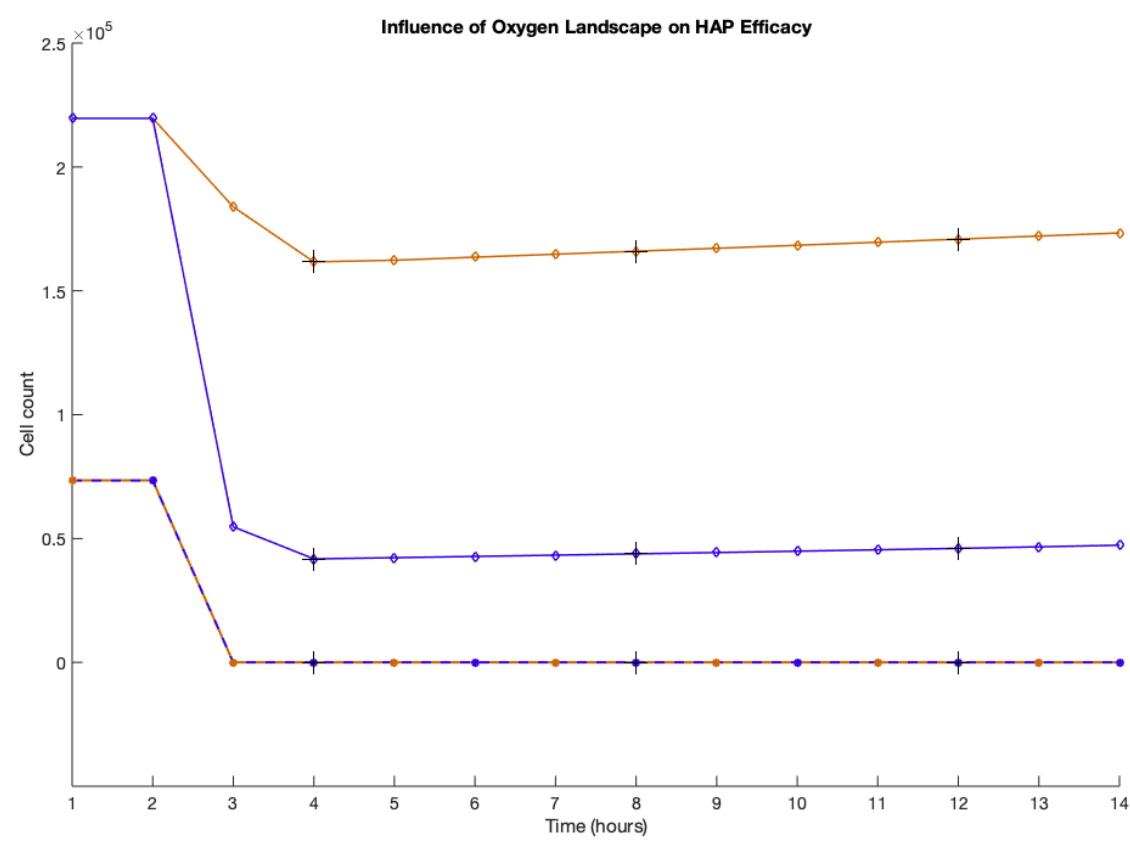

Figure 4.12: Treatment responses in MCTS A and MCTS B when HAPs are administered at 0 hours. The number of viable (undamaged) cells are plotted over time for MCTS A and MCTS B tumour. Activator cells $\left(p_{O_{2}}=0 \mathrm{mmHg}\right)$ are shown in dashed lines and bystander $\left(p_{\mathrm{O}_{2}}=100 \mathrm{mmHg}\right)$ cells shown in solid lines. Results show mean values for 10 in silico runs and '+' markers show standard deviations.

From Figure 4.12 it is clear that the bystander effects are higher in MCTS B than in MCTS A, although all activator cells are eliminated in both MCTSs. When the activator cells are spread out across the spheroid, as in MCTS B, there are more interfaces in which bystander cells experience significant bystander effects. Even if the oxygen landscape in MCTS B is highly synthetic, this in silico experiment shows that the intratumoural oxygen landscape impacts the efficacy of HAPs.

\subsection{Conclusion}

Previous in vitro and in vivo studies have validated the successfulness of HAPs in laboratory settings, however, this preclinical success has not yet been reflected in clinical trials. In an attempt to elucidate the unsatisfactory results from clinical HAP trials, we in this study investigate how oxygen-related tumour features and treatment administration plans impact the efficacy of HAP monotherapies and HAP-IR combination 


\section{HYPOXIA-ACTIVATED PRODRUGS}

therapies in silico. To this end we have developed a mathematical model capturing the spatio-temporal dynamics of tumours subjected to multimodality treatments comprising HAPs and IR. A set of results ( $i$ to $i v$ ) relating to HAP efficacy in silico have here been demonstrated.

i HAPs and IR attack tumours in different, complementary, fashions. Whilst IR provides a highly effective way to kill cancer cells in tumours, hypoxic and resting cells are significantly more resistant to IR than are well-oxygenated and actively cycling cells. HAPs, however, are alkylating agents which bioreduce in (primarily) hypoxic areas, hence HAPs primarily inflict damage in hypoxic tumour regions, which are often conferred with a high density of quiescent cells, which are susceptible to HAP damage. Consequently, HAP-IR combination treatments have the potential of produce a multifaceted attack on tumours.

ii In sufficiently hypoxic tumours, the HAP-IR treatment schedule influences treatment efficacy. However, in well oxygenated tumours the schedule is not important.

iii In sufficiently hypoxic tumours, HAP functions as a radiosensitiser and treatment intensifier, however, in well oxygenated tumours it does not.

iv Not only the overall intra-tumoural oxygenation levels, but also the intratumoural oxygen landscape, impacts HAP efficacy.

In a recent publication, Spielberg et al. [162], claim that the (lack of) clinical progress with HAP-treatments can, in great part, be attributed to the omission of hypoxia-based patient selection. This in silico demonstrates that whilst HAPs are effective treatment intensifiers for sufficiently hypoxic tumours, they have negligible effect on more well-oxygenated tumours. In simple terms: some tumours are suitable to be paired with treatment plans involving HAPs whilst others are not. In line with Spielbergs claims [162], our in silico results indicate that a personalised medicine approach is preferable if treatments involving HAPs (that are similar to TH-302) are to achieve their maximum potential in clinical settings. In this study, we qualitatively studies various aspects of HAP-IR treatment schedules using a multiscale mathematical framework. Upon the availability of in vitro and in vivo data, this mathematical framework can be calibrated in order to serve as an in silico testbed for predicting HAP-IR treatment 
scenarios. As a result of interdisciplinary collaborations, the mathematical framework used in this study has previously been validated in vitro and in vivo for applications other than HAP-IR combination treatments [31, 192]. The multiscale nature of the framework enables integration of data from various scales, be it from the subcellular scale, the cellular scale or the tissue scale. As an example of useful data, the multi cellular tumour spheroid data previously produced by Voissier et al. [3] provided our framework with calibration data for tumour growth and spatio-temporal oxygen evolution. Using existing experimental data to create data-driven mathematical models is a resourceful step involved in the advancement of mathematical oncology [51]. 
4. HYPOXIA-ACTIVATED PRODRUGS 


\section{Chapter 5}

\section{Bridging in vitro and in vivo research via agent-based modelling}

The micro-environment in an in vitro cell culture is significantly different from the micro-environment in a solid tumour in vivo. In this chapter, we use an in vitro-calibrated mathematical model to predict in vivo treatment responses to a drug that inhibits DNA damage repair.

\subsection{Chapter Summary}

Translating quantitative information between in vitro and in vivo research remains a scientifically and financially challenging step in preclinical drug development processes. However, well-developed in silico tools can be used to facilitate this in vitro to in vivo translation, and we here propose using an agent-based model to bridge the gap between in vitro and in vivo research. The agent-based model used in this chapter is governed by a set of empirically observable rules, and by adjusting only the rules when moving between in vitro and in vivo simulations, whilst keeping the fundamental mathematical model and parameters intact, the agent-based model can first be parameterised by in vitro data and thereafter be used to predict in vivo treatment responses.

As a proof-of-concept, this modelling approach is here validated against data pertaining to LoVo cells subjected to the ATR (ataxia telangiectasia mutated and rad3-related kinase) inhibiting drug AZD6738, but the modelling approach has the potential to be 


\section{BRIDGING IN VITRO AND IN VIVO RESEARCH VIA AGENT-BASED MODELLING}

expanded to numerous applications. In this chapter we also highlight how agent-based models, that are currently underutilised in pharmaceutical contexts, can be used in preclinical drug development.

\subsection{Introduction}

\subsubsection{Bridging in vitro and in vivo research}

Mathematical models, and corresponding in silico tools, can be used to simulate both in vitro and in vivo scenarios that involve cancer cell populations, or tumours, and their responses to anti-cancer treatments. However, cancer cells in an in vitro cell culture experience a microenvironment that is significantly different from the microenvironment experienced by cancer cells in a solid tumour in vivo. As these microenvironments influence cell proliferation and the delivery of oxygen, drug and nutrient molecules to cells, it follows that the dynamics of a cancer cell population in vitro differs from the dynamics of a solid tumour in vivo. Consequently, translating data obtained by in vitro experiments into quantitative information that can guide or predict in vivo experiments remains a challenging, but important, step in drug development processes.

Agent-based models (ABMs) are used in many applications in mathematical biology but are underutilised in the context of pharmaceutical drug development [193]. An ABM consists of multiple, distinct agents that may interact with each other and with their microenvironment. In this study, we introduce a novel modelling approach that uses an agent-based mathematical model to bridge the gap between in vitro and in vivo research, as is conceptually illustrated in Figure 5.1. In the ABM at the core of this modelling approach, an agent consists of one cancer cell or a group of cancer cells, and the behaviour and fate of each agent is governed by a set of empirically observable and well-established modelling rules that incorporate both intracellular and microenvironmental dynamic variables, as is thoroughly described throughout Section 5.3. To account for differences between in vitro and in vivo scenarios, the modelling rules are appropriately adjusted when moving between in vitro and in vivo simulations. By only adjusting the rules, whilst keeping the fundamental mathematical model and parameters intact, when moving between in vitro and in vivo simulations, the mathematical framework can first be parameterised by in vitro data and thereafter be used to predict in vivo treatment responses. 


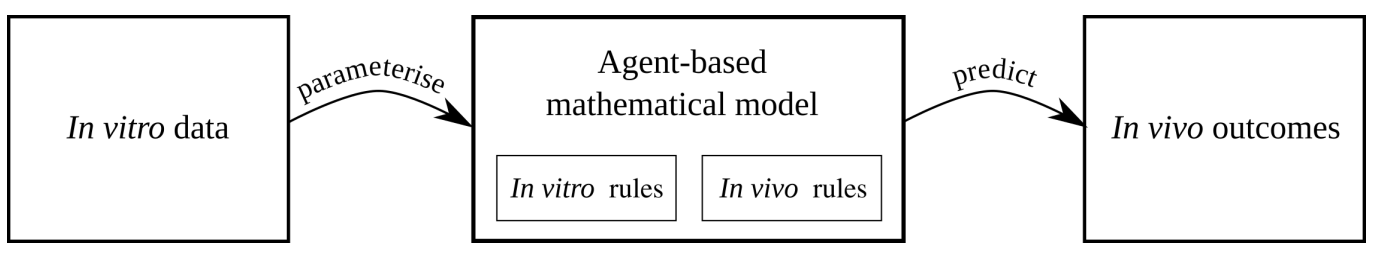

Figure 5.1: A schematic of the mathematical modelling approach used in this study. An agent-based mathematical model, that distinguishes between in vitro and in vivo modelling rules, is used to bridge the gap between in vitro and in vivo research. In this study, the mathematical model is first parameterised by in vitro data and is thereafter used to predict in vivo outcomes.

As a proof-of-concept of this modelling approach, we here simulate LoVo (human colon carcinoma) cells subjected to the anti-cancer drug AZD6738. The in vitro and in vivo data used in this work are gathered from previous work by Checkley et al. [5]. The ABM used in this study is an based on the cellular automaton (CA) introduced in Chapter 2.

\subsubsection{DNA damage response inhibiting drugs}

The deoxyribonucleic acid (DNA) in human cells is perpetually exposed to, potentially harmful, influences that can be derived from both exogenous and endogenous sources and events [194, 195]. Exogenous sources include ultraviolet radiation, ionising radiation and chemotherapeutic drugs, whilst erroneous DNA replication is an example an endogenous event yielding DNA damage [194]. Regardless of the source, a multitude of intracellular events are triggered when the DNA in a cell becomes damaged. Cells may, for example, respond to DNA damage by activating DNA repair mechanisms, cell cycle arrest or, in cases of severe DNA damage, apoptosis [196]. Such cellular responses to DNA damage are mainly governed by the DNA damage response (DDR), which comprises a complex network of signalling pathways [196]. The DDR has many functionalities and, amongst other things, it monitors DNA integrity and repairs DNA damage in order to maintain genomic stability in cells. The DDR also governs DNA replication, cell cycle progression, and apoptosis [194, 197].

When DNA repair in a cell is needed, the DDR activates relevant effector proteins [194]. Included in the group of DDR-associated effector proteins are approximately 450 proteins [197, out of which the two main regulators for cell cycle checkpoints are ataxia telangiectasia mutated kinase (ATM) and ataxia telangiectasia mutated and rad3-related kinase (ATR) [195]. ATM and ATR belong to the enzyme family 


\section{BRIDGING IN VITRO AND IN VIVO RESEARCH VIA AGENT-BASED MODELLING}

phosphatidyilinositol-3-OH-kinases (PI3K), and they both play central roles when cells respond to DNA damage [196]. In this work, we study the effects of an anti-cancer drug, namely AZD6738, that works by inhibiting ATR activity.

DNA lesions in form of single-strand breaks are a common result of replication stress, and the repair of single-strand DNA breaks is mainly attributed to ATR activity. A drug that inhibits ATR activity consequently inhibits the repair of single-strand DNA breaks post replication stress. Cancer cells are associated with high replication stress and consequently ATR inhibitors have, during the last decade, been explored as anti-cancer agents [194, 196, 198]. With the premise that inhibiting DNA damage responses should increase the effect of some other main therapy, DDR inhibitors have been explored as both radiotherapy and chemotherapy treatment intensifiers [196, 198]. Two well-studied ATR inhibitors are AZD6738 and VX-970. AZD6738 is an oral ATR inhibitor, and its anti-tumour potential has been demonstrated in preclinical vitro and in vivo xenograft studies for various ATM deficient cell lines, including ATM deficient lung cancer, chronic lymphocytic leukemia and metastatic adenocarcinoma of the colon [5, 195, 199]. Combination treatments that combine AZD6738 with either radiotherapy or chemotherapy have produced synergistic results in preclinical settings [195], and AZD6738 is currently being evaluated in clinical phase I/II trials [194, 198]. VX-970 is an intravenous ATR inhibitor [200] that has demonstrated tumour controlling effects in a phase I clinical trial, both as a monotherapy and in combination with the chemotherapy drug carboplatin [194]. A summarising table of clinical trials involving ATR-inhibitors can be found in an article by Mei et al. 198.

\subsection{Model and Method}

An ABM, specifically a CA, is used to model a population of cancer cells (in vitro), or a solid tumour (in vivo), that evolves in time and two spatial dimensions. The model describes the behaviour of cancer cells using a set of modelling rules. In order to account for differences between in vitro and in vivo scenarios, these rules are appropriately adjusted when moving between in vitro and in vivo simulations, as is described throughout Section 5.3 . Taking a minimal parameter approach, we aim to use as few rules and parameters as possible to capture the nature of the regarded system. We here chose to include model rules and parameters that pertain to the doubling time and cell cycle 
state of a cell (see Section 5.3.2, cell proliferation on the lattice (see Section 5.3.3, the distribution of oxygen and drugs across the lattice (see Sections 5.3 .4 and 5.3 .5 respectively) and cellular responses to local oxygen and drug concentrations (see Sections 5.3 .4 and 5.3.6 respectively). In this work, details concerning nutrient distribution and its effect on tumour growth are not included. Instead, under a simplifying modelling assumption, the diffusion of oxygen forms a surrogate for distribution of nutrients. Differences between the in vitro and the in vivo modelling rules are pictorially summarised in Section 5.3.8, and in vitro-calibrated model parameters are listed in Table 5.1.

The in vitro and in vivo data used in this study are gathered from previous work by Checkley et al. [5. In the in vitro experiments, populations of LoVo cells were plated and subjected to AZD6738, where population sizes of up to roughly 4000 cells were reported [5]. In the in vivo experiments, LoVo cells were subcutaneously injected in flanks of female Swiss nude mice in order to produce human tumour xenografts, and AZD6738 treatments started when the tumours had reached a volume of $0.2-0.3 \mathrm{~cm}^{3}$ [5]. Here, we regard treatment responses in terms two dynamic variables: population or tumour size and percentage of DNA-damaged (i.e. $\gamma \mathrm{H} 2 \mathrm{AX}$-positive) cells. The in vitro and in vivo data used in our current study are available in the Section 5.6 .

\subsubsection{Cellular automaton lattice}

In the model, one agent corresponds to one cancer cell (in vitro) or, due to computational costs, one group of cancer cells (in vivo). The behaviour and fate of each agent is governed by a set of rules that incorporate both intracellular and environmental dynamic variables using multiscale modelling techniques [50]. At the start of an in silico experiment, one initial agent is placed in the centre of the lattice. This initial agent produces daughter agents and ultimately gives rise to a heterogeneous population of agents. When the population has reached an appropriate size (chosen to match the in vitro and in vivo data), AZD6738 anticancer treatments commence. The CA lattice is a square lattice, and every lattice point is either empty or occupied by one agent. If a lattice point is empty, it only consists of extracellular solution (in vitro) or extracellular matrix (ECM) (in vivo). The ECM comprises multiple components such as collagen, elastin and fibronectin but we do not distinguish between these components in the model [58]. In the in vitro simulations, the dispersion of any molecules across the lattice is modelled as instantaneous, and thus the extracellular solution is considered 


\section{BRIDGING IN VITRO AND IN VIVO RESEARCH VIA AGENT-BASED MODELLING}

to render the entire lattice homogeneous in terms drug and oxygen concentrations at all times. In the in vivo simulations, however, drug and oxygen molecules are modelled as gradually diffusing over the ECM and tumour cells on the lattice, and consequently the in vivo lattice will be heterogeneous in terms of drug and oxygen concentrations. Oxygen and drug distribution across the lattice are further discussed in Sections 5.3.4 and 5.3.5 respectively. Differences between the simulated in vitro an in vivo lattices are described below.

In vitro lattice: Cell populations evolve on a two-dimensional square lattice with $100 \times 100$ lattice points, where the spacing in both spatial directions, $x_{1}$ and $x_{2}$, corresponds to one cell diameter.

In vivo lattice: Approximating the tumour as spherical, we simulate (only) a central cross section of the tumour as an, approximately circular, disk of cells living on a two-dimensional square lattice. This lattice is specifically an $\tilde{L} \times \tilde{L}=500 \times 500$ square lattice, with a spacing in both spatial directions $\tilde{x}_{1}$ and $\tilde{x}_{2}$ equal to $40 \mu \mathrm{m}$. The dimensions are chosen in order to allow our agent-based model to simulate the required physical dimensions, whilst keeping computational costs low. Post simulation time, the two-dimensional cross section of cells is extrapolated to represent a three-dimensional tumour-spheroid. This disk-to-spheroid extrapolation process is outlined in Section 5.5

\subsubsection{Cell cycle model}

In order to capture the influence of ATR and the ATR inhibitor AZD6738 on the cell cycle, we use a probabilistic, rule-based cell cycle model adapted from previous mathematical (non-agent-based) work by Checkley et al. [5]. In this model, a cancer cell progresses through various states in the cell cycle, where the states correspond to different cell cycle phases. As illustrated in Figure 5.2, a cell can be in an undamaged state $(\mathrm{G} 1, \mathrm{~S}$ or $\mathrm{G} 2 / \mathrm{M})$, a replication stress-induced DNA damaged state (D-S) or a dead state. The cause of cell death is here unrepaired replication stress. As ATR is active in the checkpoint in the intra-S phase of the cell cycle, both under undamaged circumstances and in response to DNA damage [196], ATR inhibition will inhibit the cell from progressing to the G2/M state in the mathematical cell cycle model. A cell can take different possible paths through the cell cycle, and every time the cell cycle path forks, random number generation from a uniform distribution determines which 


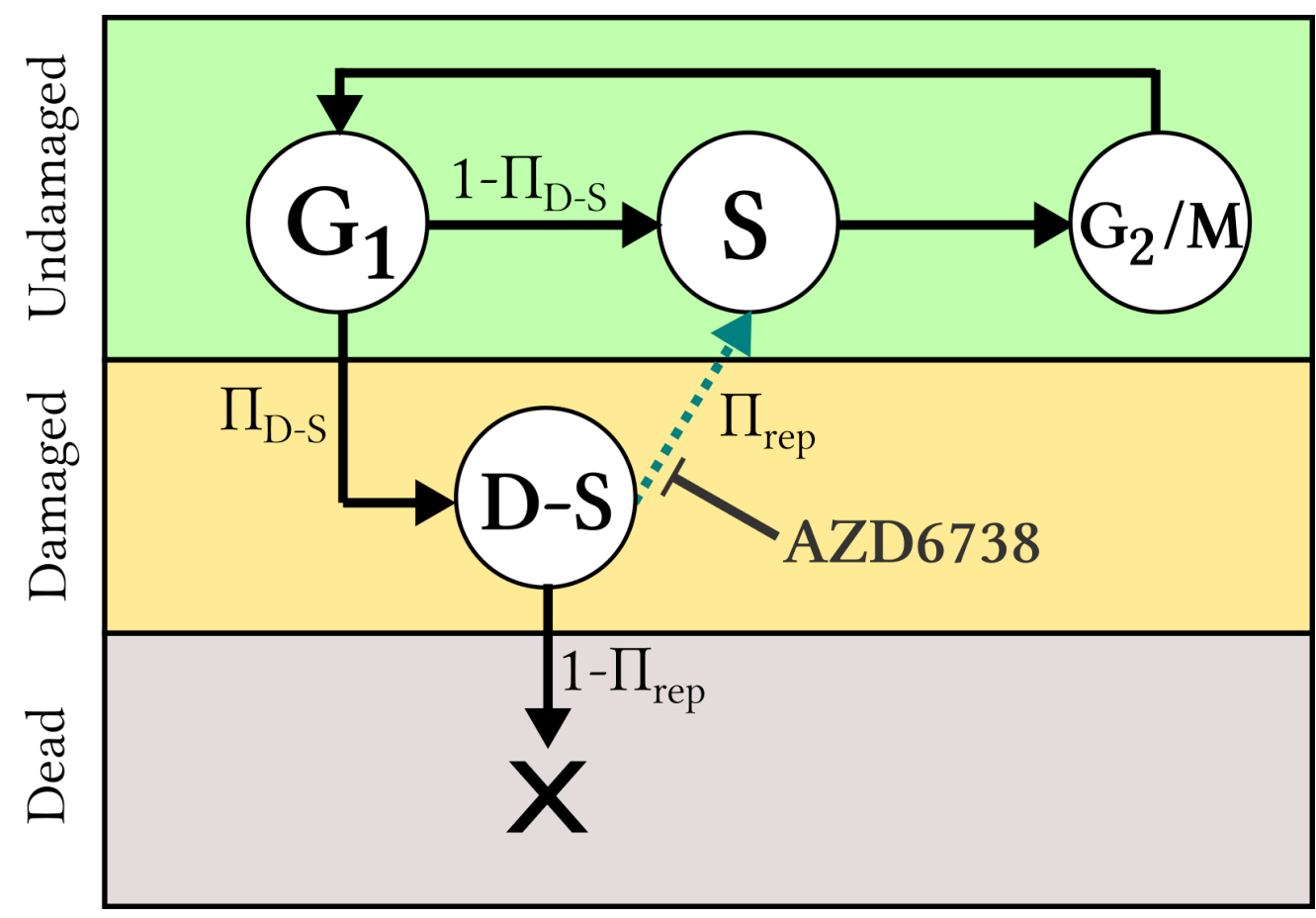

Figure 5.2: Cell cycle model: An agent, i.e a cell (in vitro) or a group of cells (in vivo), progresses through various states of the cell cycle, where the states correspond to cell cycle phases and are shown as nodes in the graph. Viable (undamaged or damaged) states are shown in circles, whilst the dead state is shown as a cross. Paths illustrate transitions between states, and symbols next to the paths denote the probabilities that the paths will be taken. The dashed path can be inhibited by an ATR inhibiting drug, such as AZD6738.

path will be taken. Every cell commences its life in the G1 state, but thereafter a cell can enter either the $\mathrm{S}$ state or the damaged S (D-S) state. The probability that a cell enters the D-S state is denoted $\Pi_{D-S}$ and is calibrated by in vitro data [5]. If a cell enters the D-S state, it has a chance to repair itself and enter the $\mathrm{S}$ state. If there is no drug in the system, this repair is always achieved, however the repair path is inhibited by the presence of the drug AZD6738. The higher the drug-concentration is, the more unlikely it is that a cell in the D-S state will successfully repair itself to the $\mathrm{S}$ state. If a cell in the D-S state can not repair, it is sentenced to die. Whether a cell in state D-S repairs or dies is decided by comparing a random number, generated from a uniform distribution, to the cell's survival probability, which is influenced by the local drug concentration $C(\bar{x}, t)$, as described in Section 5.3.6. A cell that has successfully reached the $\mathrm{S}$ state continues to the $\mathrm{G} 2 / \mathrm{M}$ state after which it duplicates and starts over in the G1 state again, ready to perform another cell cycle. 


\section{BRIDGING IN VITRO AND IN VIVO RESEARCH VIA AGENT-BASED MODELLING}

Each agent $i$ on the lattice is assigned an individual doubling time $\tau_{i}$, where $\tau_{i}$ is a random number generated from a normal distribution with mean value $\mu$ and standard deviation $\sigma$. Each agent is attributed an individual cell cycle clock, that determines when the agent should progress to a subsequent state in the cell cycle model. Progression to a subsequent state occurs once an agent has spent a certain fraction of its doubling time in its current state. The fraction of the doubling time spent in the G1, S (including D-S) and G2/M states are respectively denoted $\Theta_{G 1}, \Theta_{S}$ and $\Theta_{G 2 / M}$, where these values are approximate and chosen from literature to match values for typical human cells with a rapid doubling time of 24 hours so that $\Theta_{G 1}=11 / 24, \Theta_{S}=8 / 24$ and $\Theta_{G 2 / M}=5 / 24$ [63]. The fraction of an agent's doubling-time spent in the D-S state, $\Theta_{D-S}$, is on the other hand fitted by in vitro data produced by Checkley et al. [5. In vitro and in vivo cell cycle modelling rules are described below.

In vitro cell cycle model rules: One agent corresponds to one cancer cell that is assigned an individual doubling time $\tau_{i}$. The cell cycle path taken by cell $i$ is governed by random number generations specific to that cell.

In vivo cell cycle model rules: One agent comprises a group of identical cancer cells. Each agent is assigned an individual doubling time, $\tau_{i}$, and thus all cells belonging to agent $i$ progress simultaneously uniformly through the cell cycle model. Random number generations specific to agent $i$ determine which path the agent takes through the cell cycle.

\subsubsection{Cell proliferation}

When an agent has completed the mitosis state in the cell cycle model, a secondary agent, namely a daughter agent, is produced. Each daughter agent is placed on a random lattice point in the (approximately circular) neighbourhood of its parental agent. To accomplish circular-like growth, the model stochastically alternates between placing daughter agents on Moore and von Neumann neighbourhoods of parental agents, as is pictorially described in Chapter 2.3. A daughter agent is allowed to be placed on, up to, a $\nu$ th order neighbourhood of its parental agent, but lower order neighbourhoods (i.e. neighbourhoods closer to the parent) are prioritised and filled up first. Modelling rules concerning in vitro and in vivo cell proliferation are outlined below. 
In vitro proliferation rules: In the experimental in vitro setup, there is no spatial constraint or nutrient deficiency that is inhibiting cell division within the time-course of the experiment. Consequently cells are allowed to divide freely in the in vitro model and we set $\nu$ to be equal to infinity in the in vitro case (with the restriction that agents can not be placed outside the lattice in the in silico implementation).

In vivo proliferation rules: In vivo tumours typically consist of a core with quiescent cells and a shell of proliferating cells. To accommodate for this, a daughter agent (representing a group of daughter cells) is allowed to be placed on up to a third order (approximately circular) neighbourhood of its parental agent, so that $\tilde{\nu}=3$, in accordance with previous mathematical models [6]. For the in vivo experiment regarded in our current study, $\tilde{\nu}=3$ matches the experimental data. However, for other experiments the value of $\tilde{\nu}$ may be adjusted to fit the specific cell-line and modelling scenario at hand. When an agent is in the G1 phase of the cell cycle, it scans its environment to see if it has enough resources, in terms of space and nutrients, to commence the process of producing a daughter cell. If not, the cell enters the quiescent phase [1]. Thus in the model, when an agent is in the G1 phase, it continues to progress through the cell cycle model, provided that some free space is available on the lattice within in its $\tilde{\nu}$ th neighbourhood. If this is not the case, the agent exits the cell cycle to enter a quiescent state G0. Should neighbourhood space be made available again, here as a result of anticancer targeting, quiescent agents may re-enter the cell cycle.

\subsubsection{Oxygen distribution and influence on cells}

Tumour growth and treatment responses are highly influenced by intratumoural oxygen levels [165, 168, 171] and severely hypoxic (cancer) cells proliferate slower than do well-oxygenated cells [1].

In vitro oxygen distribution and responses: In the mathematical in vitro model, all cells are assumed to be well-oxygenated in accordance with the experimental in vitro setup performed by Checkley et al. [5]. Consequently, neither oxygen dynamics nor cellular responses to low oxygen levels are incorporated in the in vitro model.

In vivo oxygen distribution and responses: Within solid tumours, oxygen 


\section{BRIDGING IN VITRO AND IN VIVO RESEARCH VIA AGENT-BASED MODELLING}

concentrations typically vary and hypoxic regions are common tumour features [168, 169,201. Avoiding complicated details of vasculature in the model, we here approximate oxygen as diffusing in from 'outside the boundary of the tumour'. Oxygen dynamics across the CA lattice is here described using a mechanistic diffusion equation, where the oxygen concentration in location $\bar{x}$ at time $t$ is denoted by $K(\bar{x}, t)$ where

$$
\frac{\partial K(\bar{x}, t)}{\partial t}=\nabla \cdot\left(D_{K}(\bar{x}, t) \nabla K(\bar{x}, t)\right)+r_{K} m(\bar{x}, t)-\phi_{K} K(\bar{x}, t) \operatorname{cell}(\bar{x}, t)
$$

The first term in Equation 5.1 describes oxygen diffusion across the CA lattice, the second term is an oxygen supply term and the third term describes oxygen uptake by cells. Accordingly, $D_{K}(\bar{x}, t)$ denotes the oxygen diffusion coefficient, and $r_{K}$ and $\phi_{K}$ are supply and consumption coefficients respectively. The diffusion coefficient for oxygen is known from literature to be $2.5 \times 10^{-5} \mathrm{~cm}^{2} \mathrm{~s}^{-1}$ [6]. Assuming that oxygen diffuses slower over cells than in the ECM, the oxygen diffusion coefficient is divided by a factor 1.5 if there is a cell in location $\bar{x}$ at time $t$. The binary factor $m(\bar{x}, t)$ is 1 if the regarded location $\bar{x}$ is outside the tumour boundary at time $t$ and 0 otherwise, i.e. $m(\bar{x}, t)$ is 1 if the regarded lattice point is not occupied by an agent nor completely surrounded by agents. Similarly, the binary factor $\operatorname{cell}(\bar{x}, t)$ is 1 if there is a viable cell in location $\bar{x}$ at time $t$, and 0 otherwise [6]. Equation 5.1 is coupled with no-flux boundary conditions, thus the total amount of oxygen in the system fluctuates over time [67]. A scaled oxygen variable $\hat{K}(\bar{x}, t)$ is introduced in order to express oxygenation levels in percentages (\%) between $0 \%$ and $100 \%$. This scaled oxygen value is computed at every unique time step $t_{u}$ by

$$
\hat{K}\left(\bar{x}, t_{u}\right)=\frac{K\left(\bar{x}, t_{u}\right)}{\max _{\bar{x}, t_{u}} K\left(\bar{x}, t_{u}\right)} \cdot h,
$$

where $\max _{\bar{x}, t_{u}} K\left(\bar{x}, t_{u}\right)$ denotes the maximum occurring $K\left(\bar{x}, t_{u}\right)$-value at the time point $t_{u}$ [67] and $h$ is a scaling factor [6]. Low cellular oxygen levels have been shown to delay cell cycle progression by inducing arrest in, particularly, the G1 phase of the cell cycle [1] and in our model, hypoxic cells with an oxygen concentration of $10 \%$ or less display arrest (i.e. delay) in the G1 phase of the cell cycle. In mechanistic Tyson-Novak type cell cycle models [60 62, the cell cycle is governed by a system of ordinary differential equations (ODEs) in which the G1 phase is inherently elongated 
under hypoxic conditions by incorporating hypoxia-induced factors into the ODEs [6]. In this model, we use a clock to model cell cycle progression and thus we introduce a G1 delay factor (G1DF) in order to achieve a longer G1-phase under hypoxic conditions where

$$
G 1 D F(\hat{K}(x, t))= \begin{cases}2 & \text { if } 0 \% \leq \hat{K}(x, t)<1 \% \\ a_{1}+\frac{a_{2}}{a_{3}+\hat{K}(\bar{x}, t)} & \text { if } 1 \% \leq \hat{K}(x, t) \leq 10.5 \% \\ 1 & \text { otherwise }\end{cases}
$$

The G1DF is an approximation for how much the G1 phase is expanded in time as a function of oxygen concentration. It is matched to fit data points extracted from a previous mathematical study by Alarcon et al. [1], in which a Tyson-Novak cell cycle model is extended to incorporate the action of $\mathrm{p} 27$, a protein that is up-regulated under hypoxia and delays cell cycle progression. Data-fitting yields the parameter values $a_{1}=0.9209, a_{2}=0.8200, a_{3}=-0.2389$ [201]. Thus the fraction of an agent's doubling time spent in the $\mathrm{G} 1$ state is $G 1 D F(\hat{K}(\bar{x}, t)) \cdot \Theta_{G 1}$, where $G 1 D F(\hat{K}(\bar{x}, t))=1$ for normoxic cells.

\subsubsection{Drug distribution across the lattice}

Drug distribution significantly varies between in vitro and in vivo settings. In the regarded in vitro setup, the drug concentration can be regarded as homogeneous, whilst heterogeneous drug concentrations must be accounted for in vivo.

In vitro drug distribution: In the in vitro experiments performed by Checkley et al. 5, plated cell populations of roughly 1000 cells were treated with AZD6738 in the solvent dimethylsulfoxide (DMSO). In the mathematical model, we approximate the drug distribution across the CA lattice to be instantaneous (occurring at treatment time $\left.T_{0}\right)$ and homogeneous. We furthermore assume that the drug has a half-life time that exceeds the time course of the experiment, and note that there is no other drug elimination from the in vitro system. In our mathematical model, this is equivalent to there being no drug decay or elimination, hence the drug concentration $C(\bar{x}, t)$, in 


\section{BRIDGING IN VITRO AND IN VIVO RESEARCH VIA AGENT-BASED MODELLING}

location $\bar{x}$ at time $t$ is simply given by

$$
C(\bar{x}, t)= \begin{cases}0 \text { everywhere } & \text { if } t<T_{0} \\ C \text { everywhere } & \text { if } t \geq T_{0}\end{cases}
$$

where $C$ denotes the applied drug concentration (in units of molarity).

In vivo drug distribution: In the in vivo experiments performed by Checkley et al. [5], AZD6738, or vehicle in the control case, were administered via oral gavage once per day to female Swiss nude mice. In the mathematical in vivo model, we consider the drug to diffuse through the tumour from its surrounding, creating a drug gradient within the tumour. In the mathematical model, this drug dynamics is modelled using a partial differential equation (PDE), where the concentration of AZD6738 at location $\bar{x}$ at time $t$ is denoted by $C(\bar{x}, t)$ and

$$
\frac{\partial C(\bar{x}, t)}{\partial t}=\nabla \cdot\left(D_{A Z D}(\bar{x}, t) \nabla C(\bar{x}, t)\right)+p(\bar{x}, t)-\eta_{A Z D} C(\bar{x}, t),
$$

where $D_{A Z D}$ is the diffusion coefficient of the drug AZD6738, and the supply coefficient $p(\bar{x}, t)$ is greater than zero at drug administration times only for lattice points outside the tumour. Assuming first order kinetics for drug elimination, the drug decay constant $\eta_{A Z D}$ is matched to the reported half-life time of 6 hours for AZD6738 in vivo [7, 202]. Note that the drug decay term here represents all drug elimination from the system, both metabolic and that caused by excretion.

The diffusion rate of a drug is predominantly affected by the molecular size of the drug. More specifically, the diffusion coefficient of a drug is inversely proportional to the square root of the molecular weight of the drug, so that large molecules diffuse more slowly than do small molecules [7]. Using this assumption, the drug diffusion coefficient is set in relation to the oxygen diffusion coefficient, as is done in previous mathematical studies [6]. Thus the relationship between the diffusion coefficients for the drug (AZD) and oxygen $\left(\mathrm{O}_{2}\right)$ corresponds to the square of the inverse relationship between the corresponding molecular weights, such that

$$
\frac{D_{A Z D}}{D_{0_{2}}}=\frac{\sqrt{\text { molecular weight }\left(\mathrm{O}_{2}\right)}}{\sqrt{\text { molecular weight }(\mathrm{AZD})}}=\sqrt{\frac{31.998 \mathrm{~g} / \mathrm{mol}}{412.512 \mathrm{~g} / \mathrm{mol}}} \approx 0.27851
$$


where the molecular weights are collected from the PubChem database [8]. Details regarding pharmacokinetics are outside the scope of this study, bioavailability is instead calibrated using the extreme case drug scenario, as described in Section 5.4

\subsubsection{Drug responses}

AZD6738 inhibits the repair from the D-S state to the S state in the cell cycle, as illustrated in Figure 5.2, and maximal drug effect corresponds to complete repair inhibition. The drug effect is modelled using an agent-based adaptation of the sigmoid Emax model [70], in which the drug effect on a cell in position $\bar{x}$ at time $t$ is given by

$$
E(\bar{x}, t)=E_{\max } \frac{C(\bar{x}, t)^{\gamma}}{E C_{50}^{\gamma}+C(\bar{x}, t)^{\gamma}}
$$

$E_{\max }$ denotes the maximal drug effect, here corresponding to complete repair inhibition $\left(E_{\max }=1\right), E C_{50}$ denotes the drug concentration required to achieve half of the maximal drug effect, $0.5 \cdot E_{\max }$ and $\gamma$ is the Hill-exponent [70]. $E C_{50}$ and $\gamma$ are fitted from the in vitro data. When an agent is scheduled to progress from the D-S state in the cell cycle, it has a probability $\Pi_{r e p} \in[0,1]$ to repair which is determined by the local drug concentration such that

$$
\Pi_{r e p}(\bar{x}, t)=1-E(\bar{x}, t) .
$$

Note that in the absence of drugs, the repair probability is 1 . When a cell dies, it is transformed into a membrane-enclosed 'cell-corpse' [7]. In the in vivo setting, this cellular debris is digested by macrophages but in the in vitro setting such 'cell-corpses' linger on the lattice during the course of the experiment. Post the lethal event (i.e. the D-S to $\mathrm{S}$ repair failure) a cell is declared 'dead' in the model after a time $T_{L \rightarrow D}$ has passed (where $L$ stands for 'lethal event' and $D$ stands for 'death'). The parameter $T_{L \rightarrow D}$ is calibrated by in vitro experiments. The differences between modelling rules for in vitro and in vivo drug responses are described below.

In vitro drug responses: After failure to repair from the D-S state, a cell (i.e. and agent) is considered to be dead after a time $T_{L \rightarrow D}$ has passed. However, a dead cell is never physically removed from the lattice. 


\section{BRIDGING IN VITRO AND IN VIVO RESEARCH VIA AGENT-BASED MODELLING}

\begin{tabular}{|l|l|r|}
\hline Section & Parameter & Calibrated Value \\
\hline 5.3 .2 & $\mu, \sigma$ & $24 \mathrm{~h}, 0.5 \mathrm{~h}$ \\
& $\Pi_{D-S}, \theta_{D-S}$ & $0.75,0.03$ \\
\hline 5.3 .6 & $\mathrm{EC}_{50}, \gamma$ & $1 \mu \mathrm{M}, 2$ \\
& $T_{L \rightarrow D}$ & $\tau_{i}$ \\
\hline
\end{tabular}

Table 5.1: In vitro calibrated parameters.

In vivo drug responses: An agent (i.e. a group of cells) is declared to be dead and removed from the lattice after an amount of time $T_{L \rightarrow D}$ post the lethal event (failure to repair).

\subsubsection{Parameters}

The parameters used in the mathematical model are calibrated by in vitro data, this calibration process is described in Section 5.4 In the context of quantitative pharmacology, knowledge about a model's robustness is crucial [203], therefore we have provided results from the uncertainty and sensitivity analysis, as a worked example, in Chapter 6. We performed three different uncertainty and sensitivity analyses techniques, suitable for agent-based models with stochastic elements, namely $(i)$ consistency analysis, $(i i)$ robustness analysis and (iii) Latin hypercube analysis [64, 204]. Detailed descriptions of how to perform and interpret these techniques are available in Chapter 6. In accordance with the performed consistency analysis, we run 100 simulations per in silico experiment in order to formulate results (in terms of mean values and standard deviations) that mitigate uncertainty originating from intrinsic model stochasticity.

\subsubsection{Differences between in vitro and in vivo modelling rules}

Differences between the in vitro and in vivo modelling rules in the ABM are pictorially summarised in Figure 5.3

A note on simplifying modelling assumptions Note that an agent in the in vivo setting can, in general, be chosen to comprise either one cancer cell or a group of cancer cells. Note also that modelling the in vivo tumour as a (spatially) twodimensional disk means that the distribution of nutrients and drugs is modelled across a two-dimensional (rather than a three-dimensional) space. Likewise, the parameter $\tilde{\nu}$, that governs the allowed distance between a parental agent and its daughter agents, 
only concerns proliferation on a two-dimensional plane. By increasing $\tilde{\nu}$, the tumour will grow quicker and comprise a higher fraction of cycling (to quiescent) agents. Thus both the parameters $\tilde{\nu}$ and $\mu$ influence the rate of tumour growth in the in vivo simulation, and should ideally be fitted to match detailed in vivo data. In the current study, we choose to keep $\mu$ at the in vitro-calibrated value and thereafter fit $\tilde{\nu}$ to match the in vivo data. A modeller can chose to use a three dimensional spatial domain, and thus explicitly model a tumour spheroid instead of a tumour cross-section. Computational costs, fineness of available data, and the desired level of simulation details should be used to guide the choice of agents and spatial domain.

\subsubsection{Implementation}

The mathematical model is implemented in an in-house $\mathrm{C} / \mathrm{C}++$ framework. PDEs are solved using explicit finite difference methods. A flowchart of the programming code is available in Appendix A3. Simulation cell-maps are visualised using ParaView [161]. Uncertainty and sensitivity analyses are performed using MATLAB [205].

\subsection{Model Parameterisation}

Using a minimal-parameter approach, seven model parameters are calibrated using the in vitro data previously produced by Checkley et al. [5], as listed in Table 5.1. Parameter sensitivity is explored in the sensitivity analysis in Chapter 6 . The calibration process is outlined in Sections 5.4.1 through to 5.4.4. The in vivo calibration is described in Section 5.4 .5

\subsubsection{Cell doubling}

In the model, the doubling time of a cell $i$ is denoted $\tau_{i}$, where $\tau_{i}$ is stochastically picked from a normal distribution with mean value $\mu$ and standard deviation $\sigma$. Thus $\mu$ corresponds to the average cell doubling time and $\sigma$ corresponds to how synchronised the cells are. If $\sigma$ is zero, then all cells have perfectly synchronised cell cycles and duplicate at the same time. Higher $\sigma$ values achieve less synchronised cell cycles amongst cells and smoother cell count growth curves over time. The control case (i.e. no drug) cell count data is used to estimate $\mu$ and $\sigma$. 


\section{BRIDGING IN VITRO AND IN VIVO RESEARCH VIA AGENT-BASED MODELLING}

\begin{tabular}{|c|c|c|}
\hline & in vitro rules & in vivo rules \\
\hline 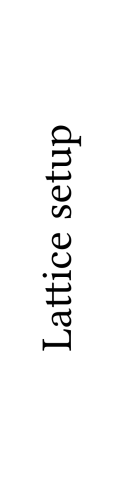 & $\begin{array}{l}\text { A monolayer of cells } \\
\text { is simulated. } \\
\text { One agent represents one cancer cell. }\end{array}$ & $\begin{array}{r}\begin{array}{r}\text { A center tumour cross- } \\
\text { section is simulated, } \\
\text { from which a tum- } \\
\text { our spheroid is } \\
\text { extrapolated } \\
\text { post sim- } \\
\text { ulation } \\
\text { time. }\end{array} \\
\text { One agent represents a group of cancer cells. }\end{array}$ \\
\hline 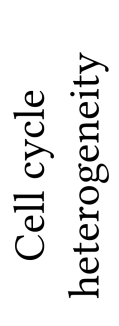 & All cells are cycling. & $\begin{array}{l}\text { Agents that are surrounded by other agents in } v \\
\text { or more orders of their circular neighbourhoods } \\
\text { are quiescent. }\end{array}$ \\
\hline 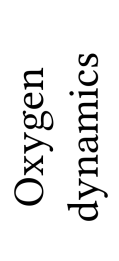 & $\begin{array}{l}\text { All cells are well oxygenated, oxygenation } \\
\text { levels are constant in time and space on the } \\
\text { lattice. }\end{array}$ & $\begin{array}{l}\text { Oxygen diffuses in from outside the } \\
\text { tumour. Hypoxia induces } \mathrm{G} 1 \text { arrest. }\end{array}$ \\
\hline 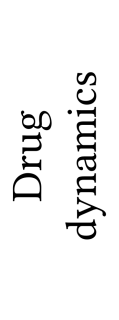 & $\begin{array}{l}\text { Drug concentration } \\
\text { Post drug administration time, drug concen- } \\
\text { tration is constant in time and space on the } \\
\text { lattice. }\end{array}$ & $\begin{array}{l}\text { Drugs diffuse in from outside the tumour, } \\
\text { and drugs are eliminated from the system. }\end{array}$ \\
\hline 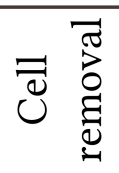 & Dead agents are not removed from the lattice. & $\begin{array}{l}\text { Dead agents are removed from the lattice after a } \\
\text { time } T_{\mathrm{L}-\mathrm{D}} \text {. }\end{array}$ \\
\hline
\end{tabular}

Figure 5.3: A summary of the differences between the in vitro and in vivo rules used in the mathematical framework. 
By observing the control case cell count data in Table 5.2, we note that the cell population roughly doubles between 2 and 24 hours (indicating an average cell doubling time, or $\mu$, of approximately 22 hours, which is less than 24 hours). Furthermore, the cell population also roughly doubles between 24 and 48 hours (indicating that $\mu$ is approximately 24 hours). However, in the last 24-hour interval, between 48 and 72 hours, the control population increases by less than $5 \%$ (indicating that $\mu$ is more than 24 hours). From these three observations, we choose to make the modelling assumption that the average doubling time for cells should be around 24 hours, and $\sigma$-values in the parameter range $[22,26]$ hours are investigated in silico. Due to the synchronised nature of the cell count data, $\sigma$-values between 0 and 2.5 hours were investigated in silico, where $\sigma=0 \mathrm{~h}$ corresponds to completely synchronised cells and $\sigma=2.5 \mathrm{~h}$ achieves a smooth cell count growth curve. After an iterative process of tuning parameters and running in silico experiments, the calibrated values are set to be $\mu=24$ hours and $\sigma=0.5$ hours. As is previously discussed, the ABM can be improved to better fit wet lab data by including variable parameter values or rules, that are updated over time. However, in the current stage of our work, we decided to fix $\mu$ and $\sigma$.

\subsubsection{Cell cycle progression}

The in vitro data provides information on how many cells are in the damaged S state via the biomarker $\gamma \mathrm{H} 2 \mathrm{AX}$. For the control case, the number of $\gamma \mathrm{H} 2 \mathrm{AX}$ positive cells in our mathematical model depends on two variables: (1) the probability $\left(\Pi_{D-S}\right)$ that a cell enters the D-S state and (2) the amount of time $\left(\Theta_{D-S} \cdot \tau_{i}\right)$ spent in the D-S state prior to repairing. Recall that $\Theta_{D-S}$ is the fraction of a cell's doubling time $\left(\tau_{i}\right)$ spent in the D-S state. As a first step, in silico experiments are performed in which we find various parameter pairs $\left(\Pi_{D-S}, \Theta_{D-S}\right)$ that agree with the control data. We thereafter note that the in vitro drug effect saturates for concentrations 3,10 and $30 \mu \mathrm{M}$ and assume that the maximal dose $(30 \mu \mathrm{M})$ yields $100 \%$ D-S to $\mathrm{S}$ repair inhibition. Thus a second step we test the variable pairs $\left(\Pi_{D-S}, \Theta_{D-S}\right)$ for this 'maximal drug and no repair' scenario in silico, and we match these in silico results to the $30 \mathrm{M}$ in vitro data. Here, we only use data from early time points (time $<12$ hours) in order to avoid the influence that dying cells have on the data and model outputs. After iterative in silico testing, the variable pair $\left(\Pi_{D-S}, \Theta_{D-S}\right)$ that best fits these both extreme cases is $\Pi_{D-S}=0.75$ and $\Theta_{D-S}=0.03$. The first extreme case refers to the 'no drug' in silico experiment 


\section{BRIDGING IN VITRO AND IN VIVO RESEARCH VIA AGENT-BASED MODELLING}

matched to the in vitro control data, where we assume that all D-S cells repair to state $\mathrm{S}$. The second extreme case refers to the 'maximum drug' in silico experiment matched to the $30 \mu \mathrm{M}$ control data, where we assume that no D-S cells repair to state S.

\subsubsection{Drug response}

Drug effects are modelled using the sigmoid E-max model [70], where the drug effect $E$ is a function of the drug concentration $C$, so that

$$
E(C)=E_{\max } \cdot \frac{C^{\gamma}}{E C_{50}^{\gamma}+C^{\gamma}},
$$

where $E_{\max }$ denotes the maximal drug effect. Here we set $E_{\max }=1$ to corresponds to total D-S to S repair inhibition. $E C_{50}$ denotes the drug concentration that achieves half of the maximal drug effect and $\gamma$ is the Hill-coefficient. If drug effect is plotted over time, the $E C_{50}$-value determines the asymptotic behaviour of the effect whilst the $\gamma$-value determines how quickly the asymptotic value is reached.

From the in vitro data, we note that the drug concentration $1 \mu \mathrm{M}$ achieves roughly half of the total drug effect in terms of $\gamma \mathrm{H} 2 \mathrm{AX}$-positive cells. (Note from Table 5.2 that when the drug concentration is $10 \mu \mathrm{M}$ or $30 \mu \mathrm{M}$, the percentage of $\gamma \mathrm{H} 2 \mathrm{AX}$-positive cells is roughly $67 \%$ at 72 hours, and when the drug concentration is $1 \mu \mathrm{M}$, the percentage of $\gamma \mathrm{H} 2 \mathrm{AX}$-positive cells is roughly $33 \%$ at 72 hours. Furthermore the lower drug concentration of $0.3 \mu \mathrm{M}$ yields a percentage of roughly $7 \% \gamma \mathrm{H} 2 \mathrm{AX}$-positive at 72 hours, and the higher drug concentration of $3 \mu \mathrm{M}$ yields a percentage of roughly $64 \%$ $\gamma \mathrm{H} 2 \mathrm{AX}$-positive at 72 hours. Consequently, we use $0.3 \mu \mathrm{M}$ as a lower bound and $3 \mu \mathrm{M}$ as an upper bound for the parameter range in which we seek $E C_{50}$, and $E C_{50}$ values in the range $[0.3 \mu \mathrm{M}, 3 \mu \mathrm{M}]$ are investigated with various Hill coefficients to fit in vitro data for all (non-control) drug concentrations. In order to avoid the impact that dying cells have on the data used parameterise $E C_{50}$ and $\gamma$, only early in vitro data (time $<12$ hours) is used to guide the calibration. After iterative in silico testing, the best variable pair $\left(E C_{50}, \gamma\right)$ is determined to be $E C_{50}=1 \mu \mathrm{M}$ and $\gamma=2$. 


\subsubsection{Cell death}

In the in vitro experiments, cells that are damaged (but not yet dead) are $\gamma \mathrm{H} 2 \mathrm{AX}$ positive. In the model, the time it takes between the 'lethal event' (i.e. a cell's failure to repair) and a cell being 'dead' is denoted $T_{L \rightarrow D}$ and is matched from the in vitro experiment. After noting the asymptotic behaviour of the in vitro data, both in terms of cell damage and cell count, we estimate that the rate of cell elimination should roughly correspond to the rate of cell production, and thus $T_{L \rightarrow D}$ should be in the same order of magnitude as the doubling time. Consequently, values of $T_{L \rightarrow D}$ between 0 and $2 \tau_{i}$ are explored in silico after which $T_{L \rightarrow D}=\tau_{i}$ is chosen as it best matches the in vitro data for all tested (non-control) drug concentrations.

\subsubsection{In vivo calibration}

For the control case, the in vivo model is directly calibrated by the in vitro data, and no further calibration is needed. For drug concentrations larger than $0 \mu \mathrm{M}$, we use the in vivo data for the highest administered drug dose to calibrate the model in order to disregard details concerning pharmacokinetics and bioavailability. In future work, our model can be integrated with pharmacokinetic modelling techniques.

\subsection{Cross-Section to Tumour Spheroid Extrapolation}

When implementing our mathematical in vivo model, only a central cross-section of the tumour is actually simulated in silico and post simulation time this cross-section area (that is approximately circular) is extrapolated to a tumour volume (that is approximately spherical). From the extrapolated tumour spheroid, the two outputs $\tilde{X}_{1}$ (percentage of $\gamma \mathrm{H} 2 \mathrm{AX}$-positive cells) and $\tilde{X}_{2}$ (tumour volume) are gathered. This is done by using simulated areas to compute the total tumour volume,

$$
\tilde{X}_{2}=\text { Total Tumour Volume }=\frac{4 \pi}{3}\left(\frac{\text { Total Simulated Area }}{\pi}\right)^{3 / 2},
$$

and the quiescent tumour volume,

$$
\text { Quiescent Tumour Volume }=\frac{4 \pi}{3}\left(\frac{\text { Quiescent Simulated Area }}{\pi}\right)^{3 / 2} .
$$




\section{BRIDGING IN VITRO AND IN VIVO RESEARCH VIA AGENT-BASED MODELLING}

From the above, the volume of cycling, or proliferating cells, is obtained by

Cycling Tumour Volume $=$ Total Tumour Volume - Quiescent Tumour Volume.

Now the output $\tilde{X}_{1}$ can be computed where,

$$
\begin{aligned}
& \tilde{X}_{1}=\text { Percentage of } \gamma \mathrm{H} 2 \mathrm{AX} \text {-positive cells in sphere }= \\
& \frac{\text { Number of simulated } \gamma \mathrm{H} 2 \mathrm{AX} \text {-positive cells }}{\text { Number of simulated cycling cells }} \times \frac{\text { Cycling Tumour Volume }}{\text { Total Tumour Volume }} .
\end{aligned}
$$

\subsection{Experimental Data}

The experimental in vitro and in vivo data used in our current study are gratefully gathered from a previous study performed by Checkley et al. [5]. In vitro data are listed in Table 5.2 and in vivo data are listed in Tables 5.3 and 5.4 .

\section{$5.7 \quad$ Results}

The mathematical framework is first parameterised by in vitro data, and is thereafter used to predict treatment responses in human tumour xenografts in vivo.

\subsubsection{Simulating in vitro experiments}

The mathematical framework is parameterised using in vitro data produced by Checkley et al. [5]. In the in vitro experiments, populations of LoVo (human colon carcinoma) cells were exposed to the ATR inhibiting drug AZD6738. The in silico results in Figure 5.4 show the evolution of the in vitro cell population over time in terms of percentage of DNA damaged, i.e. $\gamma \mathrm{H} 2 \mathrm{AX}$-positive, cells (Figure 5.4 Left) and in terms of cell count (Figure 5.4 Right). AZD6738 drugs are given at 0 hours, when the cell population has reached a size of approximately 1000 cells. Simulated response curves for six different drug concentrations, including the zero-drug concentration control case, are shown. 


\begin{tabular}{|c|c|c|c|c|}
\hline Time (hours) & Cell count & Std.Dev (count) & $\gamma \mathrm{H} 2 \mathrm{AX}$-positive (\%) & Std. Dev (\%) \\
\hline \multicolumn{5}{|l|}{$0 \mu \mathrm{M}$ (control) } \\
\hline 2 & 996 & 59.72 & 2.14 & 0.56 \\
\hline 4 & 850 & 62.30 & 2.20 & 0.45 \\
\hline 8 & 1287.5 & 417.59 & 2.90 & 1.22 \\
\hline 16 & 2742.75 & 439.69 & 1.44 & 0.33 \\
\hline 24 & 1857.5 & 409.39 & 1.29 & 0.32 \\
\hline 48 & 3605.25 & 167.38 & 1.93 & 0.44 \\
\hline 72 & 3753 & 311.17 & 1.71 & 0.21 \\
\hline \multicolumn{5}{|l|}{$0.3 \mu \mathrm{M}$} \\
\hline 2 & 1081.88 & 53.63 & 2.93 & 0.59 \\
\hline 4 & 1040.75 & 217.96 & 6.15 & 1.00 \\
\hline 8 & 1447.25 & 392.45 & 7.41 & 1.99 \\
\hline 16 & 2479.5 & 414.02 & 15.68 & 5.56 \\
\hline 24 & 1805.63 & 161.41 & 12.91 & 3.42 \\
\hline 48 & 3497.63 & 385.19 & 11.08 & 4.18 \\
\hline 72 & 3928.25 & 376.08 & 6.57 & 3.30 \\
\hline \multicolumn{5}{|l|}{$1 \mu \mathrm{M}$} \\
\hline 2 & 1129.63 & 58.26 & 17.35 & 3.31 \\
\hline 4 & 1153.63 & 331.31 & 29.12 & 3.47 \\
\hline 8 & 1303.88 & 199.72 & 36.05 & 4.35 \\
\hline 16 & 2420.25 & 744.38 & 38.51 & 9.25 \\
\hline 24 & 1226.38 & 185.58 & 45.01 & 6.01 \\
\hline 48 & 1600.38 & 456.80 & 39.47 & 7.47 \\
\hline 72 & 1612.88 & 540.55 & 33.47 & 5.46 \\
\hline \multicolumn{5}{|l|}{$3 \mu \mathrm{M}$} \\
\hline 2 & 1171.14 & 97.71 & 36.01 & 2.42 \\
\hline 4 & 1291.38 & 567.63 & 46.47 & 4.09 \\
\hline 8 & 1224.63 & 113.30 & 56.72 & 2.62 \\
\hline 16 & 1784.38 & 513.06 & 58.41 & 8.81 \\
\hline 24 & 765.75 & 70.76 & 68.07 & 2.05 \\
\hline 48 & 638.75 & 112.54 & 65.90 & 4.40 \\
\hline 72 & 392.63 & 67.64 & 63.82 & 2.67 \\
\hline \multicolumn{5}{|l|}{$10 \mu \mathrm{M}$} \\
\hline 2 & 1191.13 & 110.15 & 39.38 & 2.62 \\
\hline 4 & 1056.63 & 106.72 & 47.98 & 2.32 \\
\hline 8 & 1113.63 & 144.42 & 59.35 & 1.99 \\
\hline 16 & 1396 & 633.86 & 65.21 & 10.48 \\
\hline 24 & 654.5 & 100.26 & 71.02 & 2.10 \\
\hline 48 & 525.29 & 43.93 & 69.75 & 4.42 \\
\hline 72 & 326.63 & 47.73 & 67.25 & 2.87 \\
\hline \multicolumn{5}{|l|}{$30 \mu \mathrm{M}$} \\
\hline 2 & 1055.13 & 155.16 & 35.37 & 2.21 \\
\hline 4 & 1049.13 & 147.96 & 45.66 & 1.75 \\
\hline 8 & 1228.75 & 211.96 & 51.37 & 1.11 \\
\hline 16 & 1794.88 & 435.42 & 50.35 & 4.19 \\
\hline 24 & 629 & 27.12 & 63.92 & 2.15 \\
\hline 48 & 469.63 & 61.26 & 64.92 & 3.25 \\
\hline 72 & 265.13 & 22.26 & 67.63 & 3.96 \\
\hline
\end{tabular}

Table 5.2: In vitro data gathered from a previous study by Checkley et al. [5].

Also shown in Figure 5.4 are simulation standard deviations and in vitro data [5].

Using a minimal-parameter modelling approach, the mathematical framework is calibrated to fit in vitro data points without introducing any variable model parameters. 


\section{BRIDGING IN VITRO AND IN VIVO RESEARCH VIA AGENT-BASED MODELLING}

\begin{tabular}{|c|l|l|}
\hline Time (hours) & $\gamma \mathrm{H} 2 \mathrm{AX}$-positive (\%) & Std. Error (\%) \\
\hline $0 \mathrm{mg} / \mathrm{kg}$ (control) & 0.0320 \\
80 & 0.2770 & 0.0300 \\
96 & 0.2970 & 0.0340 \\
\hline 74 & 0.5364 \\
\hline \multicolumn{2}{|c|}{$\mathrm{g}$} & 0.3272 \\
80 & 11.3692 & 1.0949 \\
96 & 12.1945 & 0.6278 \\
\hline 74 & 14.3417 & 0.1401 \\
80 & 13.8967 & 2.7558 \\
96 & 17.4986 &
\end{tabular}

Table 5.3: In vivo data for DNA damage gathered and adapted from a previous study published by Checkley et al. [5.

\begin{tabular}{|c|c|c|}
\hline Time (hours) & Volume $\left(\mathrm{cm}^{3}\right)$ & Std. Error $\left(\mathrm{cm}^{3}\right)$ \\
\hline \multicolumn{3}{|c|}{$0 \mathrm{mg} / \mathrm{kg}$ (control) } \\
\hline 168 & 0.3028 & 0.0219 \\
\hline 264 & 0.5189 & 0.0465 \\
\hline 360 & 0.9095 & 0.0934 \\
\hline 456 & 1.3857 & 0.1554 \\
\hline 504 & 1.5646 & 0.1483 \\
\hline \multicolumn{3}{|l|}{$25 \mathrm{mg} / \mathrm{kg} \mathrm{QD}$} \\
\hline 168 & 0.3037 & 0.0342 \\
\hline 264 & 0.4411 & 0.0704 \\
\hline 360 & 0.5617 & 0.0840 \\
\hline 456 & 0.7064 & 0.1221 \\
\hline 504 & 0.8701 & 0.1187 \\
\hline \multicolumn{3}{|l|}{$50 \mathrm{mg} / \mathrm{kg} \mathrm{QD}$} \\
\hline 168 & 0.3106 & 0.0332 \\
\hline 264 & 0.3971 & 0.0768 \\
\hline 360 & 0.4002 & 0.0817 \\
\hline 456 & 0.4783 & 0.0966 \\
\hline 504 & 0.4923 & 0.0846 \\
\hline
\end{tabular}

Table 5.4: In vivo data for tumour volume gathered and adapted from a previous study published by Checkley et al. [5].

This calibration process is described in Section 5.4. Our results demonstrate that, post in vitro parameterisation, our mathematical framework is able to capture the qualitative nature of in vitro LoVo cell population growth and drug (AZD6738) responses. Figure 5.4 (Left) demonstrates that the model is able to qualitatively reproduce the asymptotic fraction of DNA damaged cells in the system but fails to match early in vitro data points. The sensitivity analysis demonstrates that the treatment timing (in relation to the overall cell cycle phase composition of the cancer cell population) notably influences treatment responses in terms of percentage of $\gamma \mathrm{H} 2 \mathrm{AX}$-positive cells. This indicates that in the in vitro experiments, a large fraction of the cells would have been in the DNA 
damaged D-S state when, or shortly after, the drug was applied. The experimental error bars in Figure 5.4 (Right) and the numerical cell count data, available in Table 5.2 , demonstrate that the doubling time of the cell population drastically decreased towards the end of the in vitro experiment and, consequently, our agent-based model was not able to replicate cell count data at 72 hours as the modelling rules and parameters were not updated over time.

\subsubsection{Simulating in vivo experiments}

Post in vitro calibration, the mathematical framework is used to simulate the in vivo experiments performed by Checkley et al. [5] in which LoVo xenografts, that are injected in mice flanks, are treated with AZD6738 once daily for 14 days. The results in Figure 5.5 show AZD6738 drug responses in terms of the percentage of DNA damaged $(\gamma \mathrm{H} 2 \mathrm{AX}$-positive) cells (Figure 5.5 Left) and tumour volume (Figure 5.5 Right). Simulated response curves to three different drug doses $(0,25$ and $50 \mathrm{mg} / \mathrm{kg}$ ) and in vivo data are provided in Figure 5.5. Figure 5.4 (Right) demonstrates that our simulated results qualitatively agrees with the in vivo results reported by Checkley et al. [5] for approximately 12 days post tumour injection for control case tumours, and for approximately 8 days post tumour injection for tumours subjected to drugs. This can be explained by the fact that the behaviour of the agents in our current model does not change over time, when in fact tumours are highly adaptable and responsive to external pressures. It follows that details pertaining to tumour growth and drug sensitivity may

vary over time, and in future work the agent-based model used in this study can be updated to incorporate variable modelling rules and parameters.

\subsection{Discussion}

In silico results obtained in this study were compared to in vitro and in vivo data and can, furthermore, be compared to previous mathematical modelling results produced by Checkley et al. [5]. In their study, Checkley et al. 5] modelled tumour 


\section{BRIDGING IN VITRO AND IN VIVO RESEARCH VIA AGENT-BASED MODELLING}
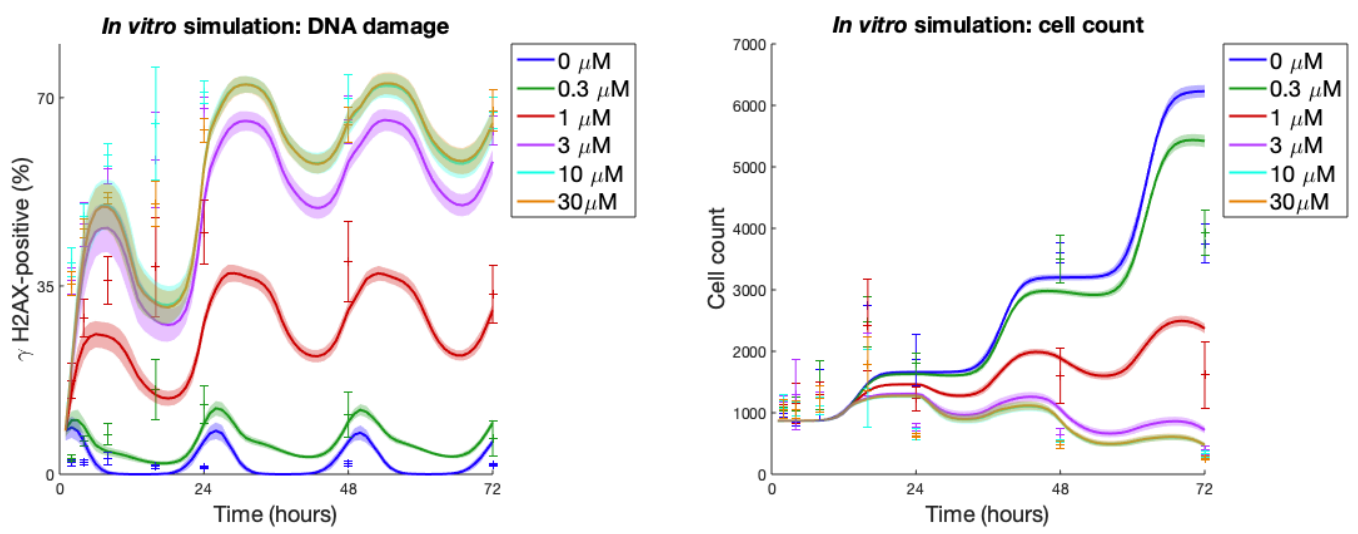

Figure 5.4: Simulated in vitro drug response curves. LoVo cells are exposed to drug (AZD6738) at 0 hours. Left: The percentage of $\gamma \mathrm{H} 2 \mathrm{AX}$-positive (DNA-damaged) cells in the system over time. Right: Cell count over time. Simulated mean values and standard deviations for 100 in silico runs are shown with solid lines and shaded ribbons respectively. In vitro mean values and standard deviations are demonstrated with center points and error bars $[5$.
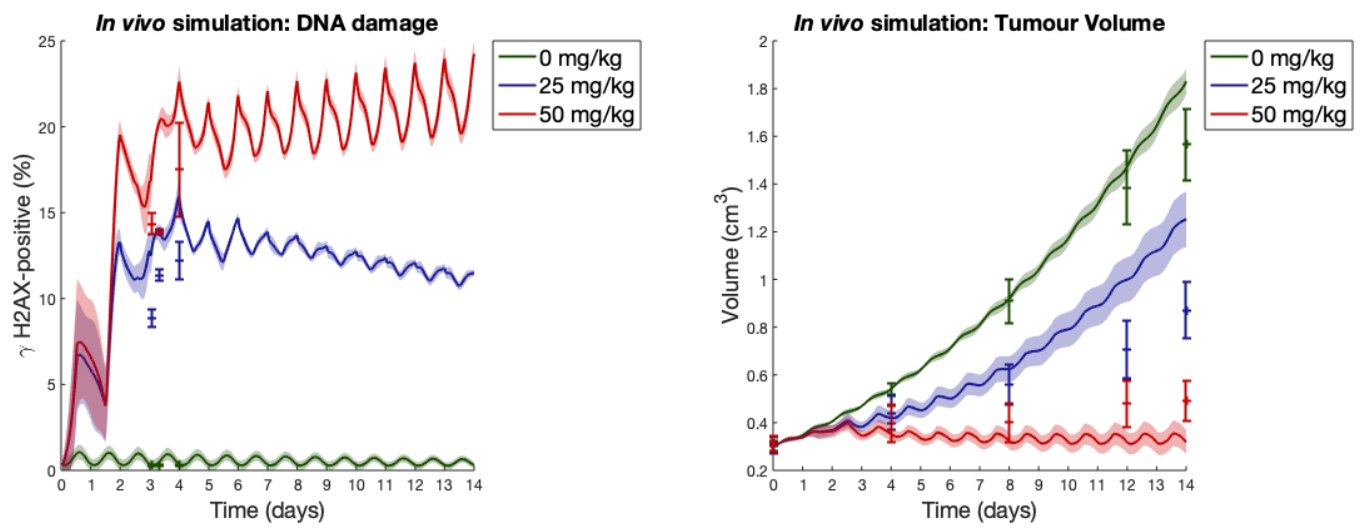

Figure 5.5: Simulated in vivo drug response curves. LoVo xenografts are exposed to drug (AZD6738) once daily for 14 days. Left: The percentage of $\gamma \mathrm{H} 2 \mathrm{AX}$-positive (DNAdamaged) cells in the xenograft over time. Right: Tumour volume over time. Simulated mean values and standard deviations for 100 in silico runs are shown with solid lines and shaded ribbons respectively. In vivo mean values and standard errors are demonstrated with center points and error bars [5].

responses to AZD6738 using coupled ordinary differential equations, where a pharmacokinetic/pharmacodynamic (PK/PD) model of tumour growth was integrated with a mechanistic cell cycle model. Their model is predictive of in vivo xenograft studies and is being used to quantitatively predict dose and scheduling responses in a clinical Phase I trial design [5]. Our modelling results qualitatively agree with those produced 
by Checkley et al. [5], although two different modelling approaches have been taken: Checkley et al. 5] regard the tumour as one entity with different compartments whilst we here use a bottom-up modelling approach and regard the tumour as consisting of multiple, distinct agents.

Moving drug-response investigations from in vitro to in vivo settings is a key step involved in the process of moving a drug from bench-to-bedside. However, in vivo data are often sparse, as gathering in vivo data is associated with practical, financial and ethical constraints. Plentiful and adaptable in silico data are, on the other hand, easy to produce, and thus sparse in vivo data can be complemented by in silico data. Consequently, mathematical frameworks, and corresponding in silico tools, can be used as an epistemic contribution to sparse data produced in wet labs. Well-formulated in silico tools can be extended to investigate various dose-schedule scenarios in order to guide in vitro and in vivo experiments. Such in silico experiments may provide a testbed for simulating various mono and combination therapies. We here propose creating ABM in silico tools in which modelling rules are based on "fundamental" principles that describe how cancer cells in a system behave (where it is up to the modeller to decide which principles should be considered "fundamental" in the specific modelling scenario at hand). The ABM considered in this study is an extension of a mathematical model that has previously been used to study tumour growth and treatment responses to chemotherapy, radiotherapy, and hypoxia-activated prodrugs [6, 186, 187]. In recent years, several ABMs have been developed for the purpose of describing various aspects of cancer dynamics [206], and it should be noted that the modelling approach proposed in Figure 5.1 is not conceptually limited to usage with the ABM described in this study. The choice of ABM should be influenced by the research question at hand, the desired level of model details and the available data.

Data-driven modeling, exploitation of existing data and proof-of-concept studies are important steps involved in current and future procedure for enabling mathematical modeling in systems medicine, as argued in a report by Wolkenhauer et al. [51. Despite the fact that mathematical modelling is becoming increasingly popular in the pharmaceutical industry, there are not that many ABMs present in the pharmaceutical scene [193]. We argue that this is a missed opportunity in the context of oncology, as ABMs 


\section{BRIDGING IN VITRO AND IN VIVO RESEARCH VIA AGENT-BASED MODELLING}

naturally capture the heterogeneous nature of tumours, which is known to complicate treatments. As multiscale ABMs organically enable the integration of data across various scales in time and space, it follows that they are useful to the interdisciplinary team that wishes to combine data and collective knowledge from its team members. Following interdisciplinary collaborations between clinicians, biologists and mathematicians, mathematical modelling may be used to enable in silico informed drug development. 


\section{Chapter 6}

\section{Uncertainty and Sensitivity Analyses}

Mathematical models of biological systems are abstractions of a highly complex reality. It follows that such models often are associated with some degree of uncertainty. In this chapter we describe a method to mitigate model uncertainty, and we discuss methods to analyse how sensitive the model is to parameter perturbations.

\subsection{Chapter Summary}

Parameters used in multiscale, agent-based models of biological systems are often associated with uncertainty. Despite this, several studies using such models omit to perform (or report) uncertainty and sensitivity analyses. There exist multiple method papers that describe how to perform uncertainty and sensitivity analyses methods, authors Alden et al. even provide a free R-based software package (SPARTAN [64]) that enables the user to perform different such methods. However, as these methods have been developed across multiple research fields, both inside and outside of the natural sciences, it is difficult to find one comprehensive review that discusses not only how to perform these methods, but also where these methods come from, and why certain conventions are proposed and/or used. To this end, we have in this chapter gathered such information for three uncertainty and sensitivity analyses techniques, namely Consistency Analysis, Robustness Analysis and Latin Hypercube Analysis. Our aim is that this will allow the reader to better evaluate uncertainty and sensitivity analyses presented by 


\section{UNCERTAINTY AND SENSITIVITY ANALYSES}

other authors, and encourage the reader to consider performing these uncertainty and sensitivity analyses methods when suitable.

\subsection{Introduction}

Parameter uncertainty in mathematical models can be derived from various origins. Epistemic uncertainty refers to uncertainty resulting from limited knowledge about the biological system at hand, whilst aleatory uncertainty stems from naturally occurring stochasticity intrinsic to biological systems [64, 207, 208]. Model parameters may thus be naturally stochastic, theoretically unknown, and unfeasible or impossible to measure precisely (or at all). Further magnifying the contributions of uncertainty in mathematical models of biological systems, in particular, is the fact that one parameter in the mathematical model may correspond to a multitude of underlying biological mechanisms and features in the real, biological system. This is especially true for minimal parameter models, i.e. mathematical models that aspire to be as non-complex as possible whilst still capturing all biological features of interest [209].

In order to understand the impact that parameter uncertainty and parameter perturbations have on results produced by a mathematical model, uncertainty and sensitivity analyses can be used. A mathematical model that comprises a set of uncertain model parameters (or inputs), is able to produce a range of possible responses (or outputs). Uncertainty analysis assesses the range of these outputs overall, and provides information regarding how certain (or uncertain) we should be with our model results, and the conclusions that we draw from them [210]. Sensitivity analysis describes the relationship between uncertainty in inputs and uncertainty in outputs. It can be used to identify which sources of input uncertainty (i.e. which model parameters) significantly influence the uncertainty in the output and, equally importantly, which do not [210]. Assessing how sensitive the output is to small input perturbations is a healthy way to scrutinise our mathematical model. Moreover, for a well-formulated model, knowledge regarding how input uncertainty influences output uncertainty can yield insight into the biological system that has not yet been empirically observed [64]. Furthermore, if the uncertainty in some input parameter is shown to not affect output uncertainty, the modeller may consider fixing that parameter, and thus reducing model complexity in accordance with a minimal-parameter modelling approach. In local sensitivity analysis 
techniques, model parameters (inputs) are perturbed one at a time whilst other parameters remain fixed at their calibrated value. In global sensitivity analysis techniques, all model parameters are simultaneously perturbed [211].

There exist several sensitivity and uncertainty analyses techniques, but here we will focus on three such techniques that are suitable to use in conjunction with agent-based mathematical models. These techniques are namely Consistency Analysis, Robustness Analysis and Latin Hypercube Analysis, which all answer important, and complementary, questions about mathematical models and their corresponding in silico responses [64, 207].

Question 1: How many data samples (i.e. how many in silico runs) are needed in order to mitigate uncertainty originating from intrinsic model stochasticity?

Answer: See Consistency Analysis.

Question 2: How robust are model reponses (outputs) to local parameter perturbations? Answer: See Robustness Analysis.

Question 3: How robust are model reponses (outputs) to global parameter perturbations? Answer: See Latin Hypercube Analysis.

Note that Consistency Analysis is only meaningful when analysing models with stochastic variables.

The statistical techniques described in this chapter have been developed and applied across multiple academic disciplines, both inside and outside of the natural sciences. Consequently, terminology and notations vary in the literature. The aim of this chapter is to combine pertinent literature from various academic fields whilst keeping terminology and mathematical notations consistent, unambiguous and tailored towards a mathematical and scientific audience. Therefore, when needed, certain algorithms from the literature are here reformulated into expressions that a mathematician would consider to be conventional. This chapter is intended to provide friendly, yet comprehensive, instructions to the modeller wanting to perform uncertainty and sensitivity analyses on agent-based models. Thorough directions on how to perform Consistency 


\section{UNCERTAINTY AND SENSITIVITY ANALYSES}

Analysis (Section 6.4), Robustness Analysis (Section 6.5) and Latin Hypercube Sampling and Analysis (Section 6.6) are provided. Consistency Analysis utilises the measure of stochastic superiority, which is therefore discussed in Section 6.3. Throughout this chapter, we have included some historical information that elucidates why certain statistical conventions are used. For the lazy busy reader, each section also contains pictorial, step-by-step instructions on how to perform the aforementioned techniques.

\subsection{The Measure of Stochastic Superiority}

\subsubsection{The Common Language Statistic}

In 1992, McGraw and Wong introduced the common language statistics $(C L)$ as an intuitive way to compare two distributions of data [212]. The $C L$ was initially introduced as a tool to compare data from normal distributions, but was later on approximated for use on any continuous distributions. The $C L$ describes the probability that a random data sample from one of the distributions is greater than a random data sample from the other distribution. For example, if we have two continuous data distributions $B$ and $C$, and we are comparing the distributions with respect to some variable $X$, then the $C L$ is simply given by

$$
C L_{B C}(X)=P\left(X_{B}>X_{C}\right)
$$

where standard probability notations have been used so that $P\left(X_{B}>X_{C}\right)$ denotes the probability that a random data sample $X_{B}$ from distribution $B$ is greater than a random data sample $X_{C}$ from distribution $C$ [212]. Thus the subscript of $X$ here signifies the distribution from which the data sample $X$ was taken.

\subsubsection{The A Measure of Stochastic Superiority}

The $C L$ was developed to compare continuous data distributions, but Vargha and Delaney [213] introduced the A measure of stochastic superiority (or A-measure for short) as a generalisation of the $C L$ that can directly be applied to compare both continuous and discrete distributions of variables that are at least ordinally scaled. When comparing two distributions $B$ and $C$, with respect to the variable $X$, the A-measure $A_{B C}(X)$ 
is given by

$$
A_{B C}(X)=P\left(X_{B}>X_{C}\right)+0.5 P\left(X_{B}=X_{C}\right),
$$

where $P\left(X_{B}=X_{C}\right)$ denotes the probability that a random data sample from distribution $B$ is equal to a random data sample from distribution $C$. By comparing Equations 6.1 and 6.2 , it is clear that in the continuous case, where $P\left(X_{B}=X_{C}\right)=0$, the A-measure reduces to the $C L$.

If two distributions that are identical with respect to the variable $X$ are compared, then $P\left(X_{B}>X_{C}\right)=P\left(X_{C}>X_{B}\right)$ and we say that the distributions $B$ and $C$ are stochastically equal with respect to the variable $X$. On the other hand, if $P\left(X_{B}>X_{C}\right)>P\left(X_{C}>X_{B}\right)$, then we say that the distribution $B$ is stochastically greater than distribution $C$, and accordingly, that distribution $C$ is stochastically smaller than distribution $B$ [213]. If distribution $B$ is stochastically greater than distribution $C$ with respect to the variable $X$, it simply occurs more often that the sample $X_{B}$ is greater than the sample $X_{C}$ when two random samples $X_{B}$ and $X_{C}$ are compared. Likewise, if distribution $C$ is stochastically smaller than distribution $B$ with respect to the variable $X$, it occurs more often that the sample $X_{C}$ is smaller than the sample $X_{B}$ when comparing two random samples $X_{B}$ and $X_{C}$. These definitions of stochastic relationships (stochastically equal to, stochastically greater than, stochastically smaller than), used by Vargha and Delayney [213], amongst others, are weaker than definitions used by some other authors, but sufficient and appropriate for our current purposes: comparing distributions of discrete data samples produced by in silico simulations based on stochastic, individual-based mathematical models.

When comparing two samples $X_{B}$ and $X_{C}$, the possible outcomes are (i) that $X_{B}$ is greater than $X_{C}$, (ii) that $X_{B}$ is equal to $X_{C}$ and (iii) that $X_{B}$ is smaller than $X_{C}$. These three possible outcomes must sum up to one so that,

$$
P\left(X_{B}>X_{C}\right)+P\left(X_{B}=X_{C}\right)+P\left(X_{C}>X_{B}\right)=1 .
$$

In the continuous case, $P\left(X_{B}=X_{C}\right)=0$ as previously stated, and thus it follows 


\section{UNCERTAINTY AND SENSITIVITY ANALYSES}

that

$$
P\left(X_{C}>X_{B}\right)=1-P\left(X_{B}>X_{C}\right), \text { for continuous distributions, }
$$

and thus it suffices to know only one of the values $P\left(X_{B}>X_{C}\right)$ or $P\left(X_{C}>X_{B}\right)$ in order to determine the stochastic relationship between the distributions $B$ and $C$ with respect to $X$.

- Example 6.3.2a: If $P\left(X_{B}>X_{C}\right)=0.4$, then it is clear that $P\left(X_{C}>X_{B}\right)=0.6$ and thus that $P\left(X_{B}>X_{C}\right)<P\left(X_{C}>X_{B}\right)$, or equivalently, that distribution $B$ is stochastically smaller than distribution $C$.

However in the discrete case, $P\left(X_{B}=X_{C}\right)$ is not generally equal to zero and therefore,

$$
P\left(X_{C}>X_{B}\right)=1-P\left(X_{B}>X_{C}\right)-P\left(X_{B}=X_{C}\right) \text { for discrete distributions. }
$$

Consequently, one single value $P\left(X_{B}>X_{C}\right)$ or $P\left(X_{C}>X_{B}\right)$ alone can generally not be used to determine the stochastic relationship between the distributions $B$ and $C$.

- Example 6.3.2p: If, again, $P\left(X_{B}>X_{C}\right)=0.4$, it follows that $P\left(X_{C}>X_{B}\right)=$ $0.6-P\left(X_{B}=X_{C}\right)$. This does not give us enough information to determine the stochastic relationship between the two distributions $B$ and $C$.

In order to proceed to compare the distributions $B$ and $C$ in this case, the stochastic difference $\delta$ is introduced where $\delta$ is given by

$$
\delta=P\left(X_{B}>X_{C}\right)-P\left(X_{C}>X_{B}\right), \quad \delta \in[-1,1] .
$$

Via a linear transformation, the transformed stochastic difference, $\delta^{\prime} \in[0,1]$, can be obtained using Equation 6.5 so that 


$$
\begin{aligned}
\delta^{\prime}=\frac{\delta+1}{2} & =\frac{P\left(X_{B}>X_{C}\right)-P\left(X_{C}>X_{B}\right)+1}{2}= \\
= & \frac{P\left(X_{B}>X_{C}\right)-\left(1-P\left(X_{B}>X_{C}\right)-P\left(X_{B}=X_{C}\right)\right)+1}{2}= \\
& =P\left(X_{B}>X_{C}\right)-0.5 P\left(X_{B}=X_{C}\right)=A_{B C}(X)
\end{aligned}
$$

from which we can see that the A-measure, $A_{B C}(X)$ (Equation 6.2), measures the stochastic difference between $P\left(X_{B}>X_{C}\right)$ and $P\left(X_{C}>X_{B}\right)$ under a linear transformation [213].

In order to estimate the A-measure using samples from two distributions, the point estimate of the A-measure, here denoted the $\hat{A}$-measure (with a hat), is used (in the SPARTAN package [64], this is referred to as the A test score). For example, if we want to compare two discrete distributions $B$ and $C$, where $B$ comprises $m$ data samples (of some variable $X$ ) so that $B=\left\{b_{1}, b_{2}, . ., b_{m}\right\}$ and $C$ comprises $n$ data samples (of some variable $X$ ) so that $C=\left\{c_{1}, c_{2}, . ., c_{n}\right\}$ then

$$
\hat{A}_{B C}(X)=\frac{\#\left(b_{i}>c_{j}\right)}{m n}+0.5 \frac{\#\left(b_{i}=c_{j}\right)}{m n}
$$

where $i=1,2, . ., m$ and $j=1,2, . ., n$ and \#(event) is the 'counting function' that simply denotes the number of times that a certain event occurs when comparing all possible pairs of data samples $\left(b_{i}, c_{j}\right)$. For clarity, Figure 6.1 provides an example of how the $\hat{A}$-measure can be computed by counting events.

Using more conventional mathematical notation, the $\hat{A}$-measure is given by

$$
\hat{A}_{B C}(X)=\frac{1}{m n} \sum_{i=1}^{m} \sum_{j=1}^{n} H\left(b_{i}-c_{j}\right),
$$




\begin{tabular}{|c|c|c|c|c|c|c|c|}
\hline \multicolumn{7}{|c|}{$\begin{array}{l}B=\left\{b_{1}, b_{2}, b_{3}, b_{4}, b_{5}\right\}=\{1,2,3,2,1\} \\
C=\left\{c_{1}, c_{2}, c_{3}, c_{4}, c_{5}\right\}=\{6,0,0,2,1\}\end{array}$} & \\
\hline & & & & & & $5^{405}$ & \\
\hline$\#\left(b_{i}>c_{j}\right)$ & 2 & 3 & 4 & 3 & 2 & 14 & \\
\hline$\#\left(b_{i}=c_{j}\right)$ & 1 & 1 & 0 & 1 & 1 & 4 & \\
\hline$\#\left(b_{i}<c_{j}\right)$ & 2 & 1 & 1 & 1 & 2 & 7 & \\
\hline$\hat{A}_{B C}(X)=14$ & & & & & & & \\
\hline
\end{tabular}

Figure 6.1: Using Equation 6.8 to compute the point estimate of the A-measure, i.e the $\hat{A}$-measure or $\hat{A}_{B, C}$, of the two distributions of data samples $B$ and $C$ with sizes $m$ and $n$ respectively.

where $H(x)$ is the Heaviside step function such that

$$
H(x)=\left\{\begin{array}{cc}
1 & \text { for } x>0 \\
\frac{1}{2} & \text { for } x=0 \\
0 & \text { for } x<0
\end{array}\right.
$$

If $\hat{A}_{B C}(X)=0.5$, then the distributions $B$ and $C$ are stochastically equal with respect to the variable $X$. The $\hat{A}$-measure can thus be used to measure 'how equal' two discrete distributions $B$ and $C$ are, by assessing how much the $\hat{A}$-measure $(\in[0,1])$ deviates from equality, i.e. the value 0.5 . The closer the $\hat{A}$-measure is to 0.5 , the 'more equal' the two compared distributions are [213]. In many applications, we are only interested in 'how equal' two distributions $B$ and $C$ are, and it is not important which distribution is the stochastically greater one. In such cases we are only interested in how much the $\hat{A}$-measure deviates from stochastic equality (i.e. the value 0.5 ) but the direction is not important. Or in mathematical terms: the magnitude of the difference between the $\hat{A}$-measure and stochastic equality is important but the sign is not. The magnitudal $\hat{A}$-measure, here denoted $\underline{\hat{A}}$ with an underscore, ignores the sign of deviation 
from equality and is given by

$$
\underline{\hat{A}}= \begin{cases}\hat{A}_{B C}(X) & \text { if } \hat{A}_{B C}(X) \geq 0.5, \\ 1-\hat{A}_{B C}(X) & \text { if } \hat{A}_{B C}(X)<0.5\end{cases}
$$

The statistical significance is used to describe the effect of the stochastic difference between two distributions $B$ and $C$. If two distributions $B$ and $C$ are 'fairly equal' (i.e. if they yield an $\underline{\hat{A}}_{B C}$-measure close to 0.5$)$ then the statistical significance is classified as small. The statistical significance is classified using the magnitudal $\underline{\hat{A}}$-measure and, using guidelines from Vargha and Delaney [213], the statistical significance is classified to be small, medium or large with respect to $X$ according to the following threshold values for $\underline{\hat{A}}_{B C}(X)$,

$$
\text { Statistical Significance }= \begin{cases}\text { small } & \text { if } \underline{\hat{A}}_{B C}(X) \in[0.5,0.56], \\ \text { medium } & \text { if } \underline{\hat{A}}_{B C}(X) \in(0.56,0.64] \\ \text { large } & \text { if } \underline{\hat{A}}_{B C}(X) \in(0.64,0.71] .\end{cases}
$$

These threshold values (that might appear somewhat arbitrary) were first introduced by psychologist and statistician Cohen [214, 215] in the 1960s when comparing normal distributions, but then in terms of another statistical measurement: the effect size (Cohen's) d where

$$
\mathbf{d}=\frac{\mid(\text { mean of population } B)-(\text { mean of population } C) \mid}{\sigma},
$$

and $\sigma$ is the standard deviation of either $B$ or $C$ (as $\mathrm{B}$ and $\mathrm{C}$ here are assumed to have the same standard deviation) [215, 216]. Omitting details from statistics, a small d-value essentially corresponds to a big overlap between distributions $B$ and $C$, whilst a large $\mathbf{d}$-value corresponds to a small overlap between distributions $B$ and $C$, as is illustrated in Figure 6.2. Cohen decided to use the threshold $\mathbf{d}$-values for describing 'small', 'medium' and 'large' effect sizes to be $0.2,0.5$ and 0.8 respectively [215]. If we hold on to the assumption that $B$ and $C$ are two normal distributions with the same variability, and furthermore say that they contain the same number of data samples, we can use measures of overlap to get a further 'feel' for the previously discussed effect sizes, as illustrated in Figure 6.2. Cohen's $\mathbf{d}$ value can also be converted into 'the probability that a random data sample $X_{B}$ from (normal) distribution $B$ is larger than a random 

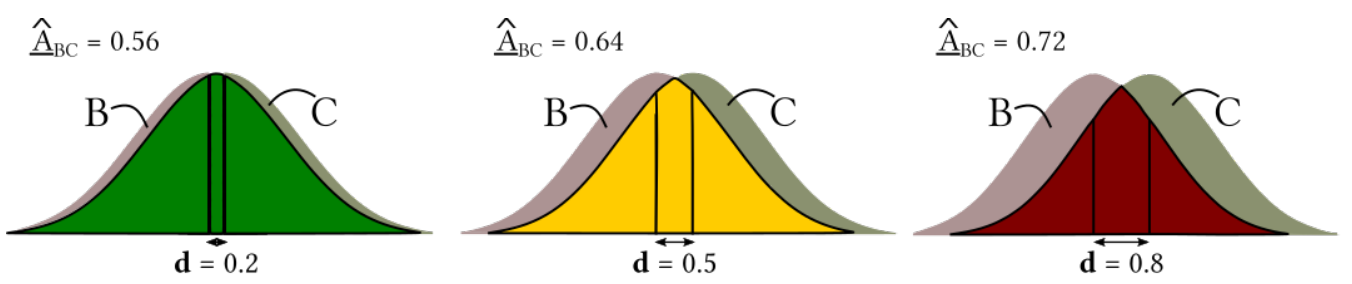

Figure 6.2: The small (left), medium (centre) and large (right) threshold values for the scaled A measure of stochastic superiority $\left(\underline{\hat{A}}_{B C}\right)$ are based on Cohen's d-values comparing two normal distributions $B$ and $C$ with the same variance. The higher the overlap between $B$ and $C$, the smaller the $\mathbf{d}$-value, and the smaller the $\underline{\hat{A}}_{B C}$-measure $\left(\underline{\hat{A}}_{B C} \in[0.5,1]\right)$.

data sample $X_{C}$ from (normal) distribution $C$ [212], but that is exactly what the $\hat{A}$ measure $\hat{A}_{B C}(X)$ measures! So this is where the threshold values for the descriptors 'small', 'medium' and 'large' statistical differences listed in Equation 6.12 come from.

Now, Cohen motivated his choice of the $\mathbf{d}$-value thresholds using a blend of intuitive 'everyday' examples and mathematical reasoning [215], but he did issue a warning regarding the fact that the threshold values should be determined based on the research methodology at hand. Thus the (modeller) should not blindly use Cohen's suggested thresholds, but instead reason what constitutes a small enough statistical significance in the study at hand. The (modeller) must also decide how fine the data samples in the data distributions should be before performing consistency analysis. In many applications, it is likely the amount of data samples required in order to achieve a small statistical significance increases with the fineness of the data. Nonetheless, scientific conventions are useful (no need for citations) and thus in the remainder of this chapter we will use the threshold values suggested by Cohen, as is done in other mathematical biology studies [64.

\subsection{Consistency Analysis}

In silico simulations based on mathematical models with built-in stochasticity will not produce the same output data every simulation run. Consistency Analysis (also called aleatory analysis) is a stochastic technique that answers the question: how many data samples do we need to produce in order to mitigate uncertainty originating from intrinsic model stochasticity? In our case, one data sample is the product of one in silico simulation, so an equivalent question is: how many in silico simulations should we run 
before describing our results in terms of for example average values, standard deviations or similar?

Let us say that one in silico simulation produces one data sample of some output variable $X$. This data sample can for example correspond to 'the population size at time point $T^{\prime}$, or something similar. It is up to the modeller to identify and decide what meaningful output variable(s) should be, and consistency analyses can be performed on multiple output variables at multiple time steps, for comprehensiveness. Before we begin, note that, when performing Consistency Analysis, we always use the calibrated model parameters.

The first step involved in performing Consistency Analysis is to produce multiple distributions of data of various sizes. We say that a distribution with $n$ data samples has a distribution-size $n$, and the goal of Consistency Analysis is to find the smallest $n$ value (here denoted $n *$ ) that yields a small stochastic significance. To do this, we create various distribution groups that all contain 20 distributions each of some distributionsize $n$, as is shown in Step 1 in Section 6.4.1. Following the methodology described in previous work by Alden et al. [64, we create one distribution group that contains 20 distributions of size $n=1$, one distribution group that contains 20 distributions of size $n=5$ and so on. Here, the $n$-values 1, 5, 50, 100 and 300 are evaluated [64] and thus we must produce a total of $20 \cdot(1+5+50+100+300)=9120$ in silico runs. (Note that, if the desired accuracy is not achieved for the highest investigated $n$-value, here $n=300$, higher values of $n$ can be explored).

We here let a distribution $D_{n, k}$ denote the $k$-th distribution of distribution size $n$ so that

$$
D_{n, k}=\left\{d_{n, k}^{1}, d_{n, k}^{2}, \ldots, d_{n, k}^{n}\right\}
$$

where $d_{n, k}^{h}$ is the the $h$-th data sample in distribution $D_{n, k}$ and $h=1,2, . ., n$. The $\hat{A}$-measure resulting from comparing two distributions $D_{n, k}$ and $D_{n, k^{\prime}}$ with respect to the variable $X$ is denoted by $\hat{A}_{k, k^{\prime}}^{n}(X)$.

Now, within every distribution-group, we compare the first distribution $(k=1)$ to 
all other distributions $\left(k^{\prime}=2,3, . ., 20\right)$ using the $\hat{A}$-measure. This yields $19 \hat{A}$-measures per distribution-group (as is shown in Step 2 in Section 6.4.1. The maximum scaled $\hat{A}$-measure with respect to $X$, occurring in a distribution-group $g$ that contains distributions of size $n_{g}$, is denoted $\underline{\hat{A}}_{\text {max }}^{n_{g}}(X)$. The smallest value $n_{g}$ for which $\underline{\hat{A}}_{\text {max }}^{n_{g}}(X) \leq 0.56$ is denoted $n *$. In other words: $n^{*}$ corresponds to the smallest distribution-size for which all of the 19 computed $\hat{A}$-measures yield a small stochastic significance, as is shown in Step 3 in Section 6.4.1. This answers the question that we set out to answer via Consistency Analysis: $n^{*}$ data samples (or in silico runs) are needed in order to mitigate uncertainty originating from intrinsic model stochasticity. The procedure on how to perform Consistency Analysis is outlined Section 6.4.1.

\subsubsection{Quick Guide to Consistency Analysis}

Here follows a quick guide for how to perform Consistency Analysis.

Consistency Analysis answers Question 1: How many data samples (or in silico runs) are needed in order to mitigate uncertainty originating from intrinsic model stochasticity? 
1) Produce multiple groups of distributions. Each group (g) contains 20 distributions comprising $\mathrm{n}_{\mathrm{g}}$ data samples each, as illustrated below.

We let $\mathrm{D}_{\mathrm{n}, \mathrm{k}}$ denotes the $\mathrm{k}$ :th distribution of distribution size $\mathrm{n}$ so that

$$
D_{n, k}=\left\{d_{n, 1}^{1}, d_{n, 2}^{2}, . ., d^{n-1}{ }_{n, 19}, d_{n, 20}^{n}\right\} .
$$
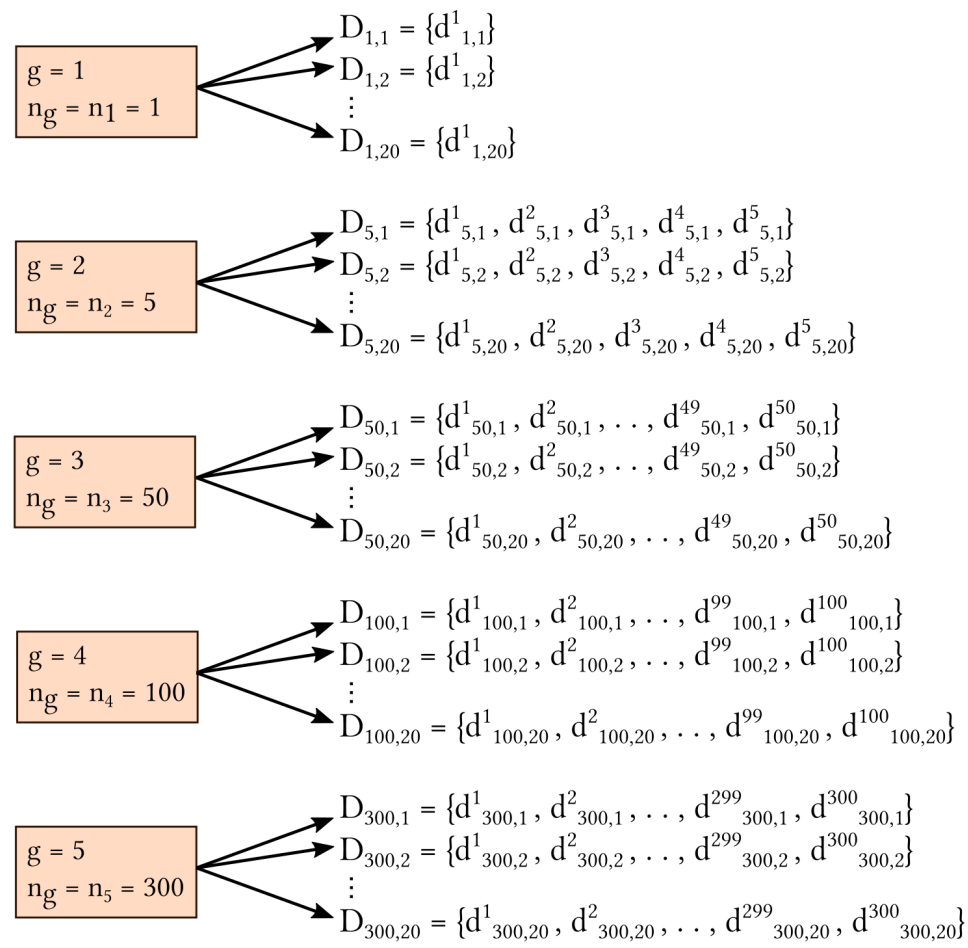

We thus need to produce $20 \sum \mathrm{n}_{\mathrm{g}}$ data samples, here $20(1+5+50+100+300)=$ $=9120$ data samples. 


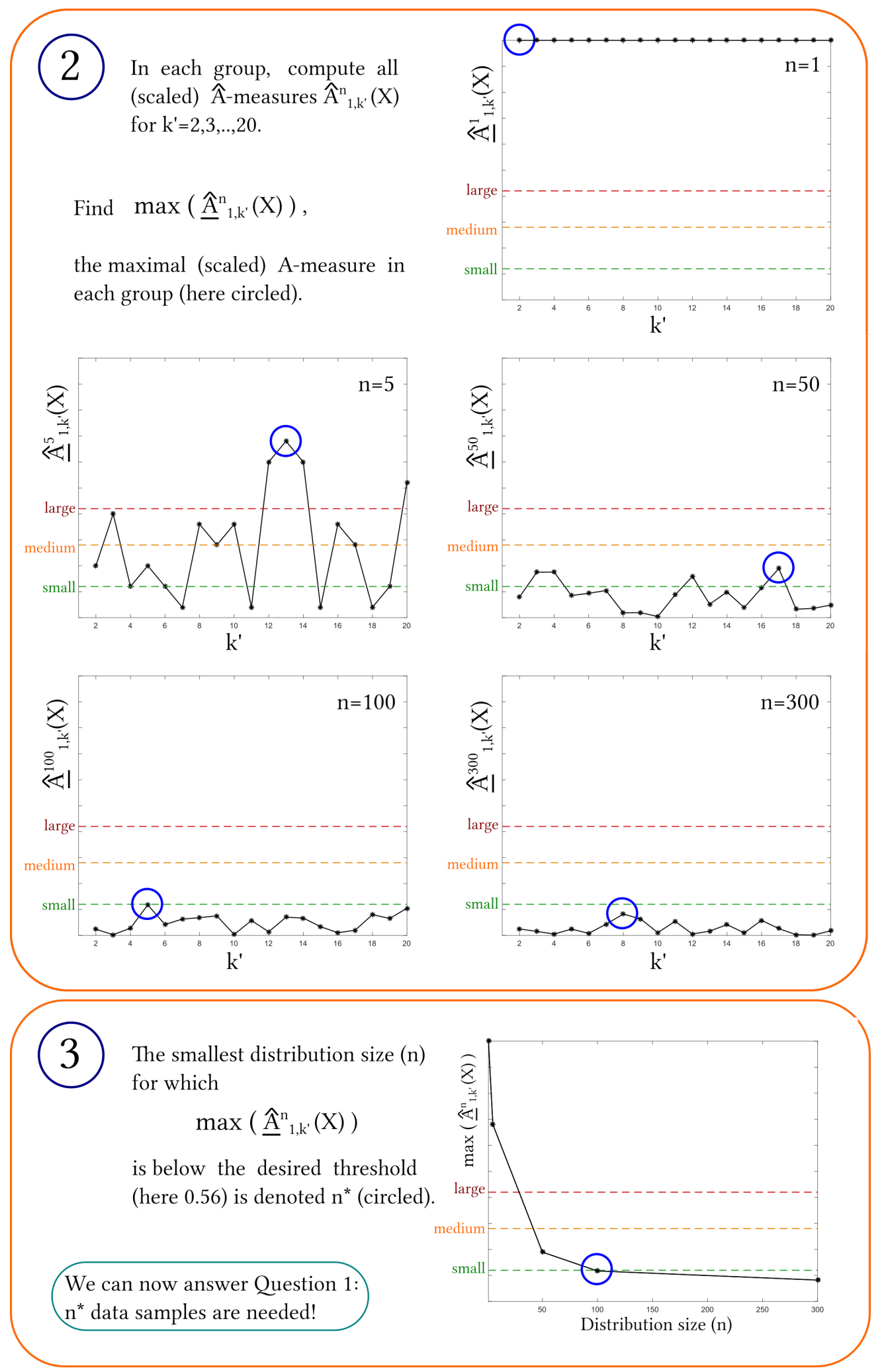




\section{A few remarks:}

- Consistency Analysis can be performed on one or multiple output variables X.

- When performing Consistency Analysis, the calibrated model parameters are used.

- The threshold values for small, medium and large statistical significance can be adjusted if appropriate.

Figure 6.3: 3 -step quick guide on how to perform Consistency Analysis.

\subsection{Robustness Analysis}

Robustness Analysis answers the question: how robust are model responses to local parameter perturbations? Robustness Analysis investigates if, and how, perturbing the value of one input parameter significantly changes an output $X$. Using the $\hat{A}$-measure, data distributions containing output data produced by perturbed input parameters, are compared to a data distribution containing output data produced by the calibrated input parameters. All perturbed data distributions are here of size $n^{*}$, where $n^{*}$ is decided in the Consistency Analysis process, previously described in Section 6.4, when analysing stochastic models.

Before commencing the Robustness Analysis, we must identify the uncertain model parameters that we want to investigate the robustness of. We denote these parameters $p^{i}$, where $i=1,2, . ., q$, and thus we have a total of $q$ parameters whose robustness we will investigate. Now, as illustrated in Step 1 in Section 6.5.1, we let each such parameter $p^{i}$ be investigated at $r\left(p^{i}\right)$ different parameter values (including the calibrated value), and thus we need to generate a total of $P$ distributions of sample size $n^{*}$ where

$$
P=\sum_{i=1}^{q} r\left(p^{i}\right) .
$$

Note that, the number of investigated parameter values, $r\left(p^{i}\right)$, need not be the same for every input parameter $p^{i}$. Investigated distributions of sample size $n^{*}$ are here denoted $D_{n^{*}, p_{j}^{i}}$, where $i=1,2, . ., q$ denotes which parameter is being perturbed and $j=$ $1,2, . ., r\left(p^{i}\right)$ denotes the specific perturbation of parameter $p^{i}$. For some perturbation $j=C$, the parameter value $p_{j}^{i}$ equals the calibrated value for input parameter $p^{i}$. 


\section{UNCERTAINTY AND SENSITIVITY ANALYSES}

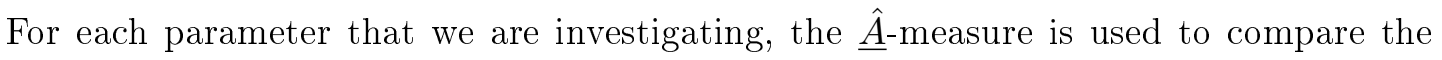
calibrated distribution $D_{n^{*}, p_{C}^{i}}$ to all distributions $D_{n^{*}, p_{j}^{i}}$. Note that, when $j=C$, the calibrated distribution is compared to itself and thus the $\underline{\hat{A}}$-measure equals 0.5 . These

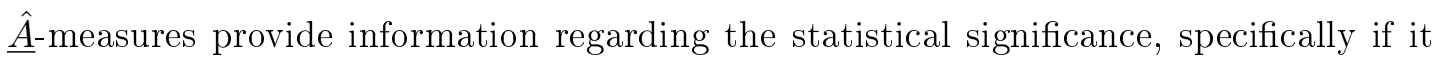
can be described to be small, medium or large under parameter perturbations. Plotting the corresponding $\underline{\hat{A}}$-measure over the parameter value $p_{j}^{i}$ for each parameter $p^{i}$, paints an informative picture of local parameter robustness, as shown in Step 2, in Section 6.5.1. Another descriptive way to demonstrate the influence that parameter values $p_{j}^{i}$ have on some output variable $X$ is to use boxplots. As is illustrated in Step 3 in Section 6.5.1, boxplots can be used to clearly show the median, different percentiles, and outliers of some data distribution $D_{n^{*}, p_{j}^{i}}$ as a function of the parameter value $p_{j}^{i}$. The methodology to perform Robustness Analysis is outlined in Section 6.5.1. Note that Robustness Analysis does not pick up on any non-linear effects, between an input parameter $p^{i}$ and an output $X$, that occur when more than one model parameter is simultaneously perturbed [211]. Such effects can however be identified using a global sensitivity analysis technique, such as Latin Hypercube Analysis, as described in Section 6.6 .

\subsubsection{Quick Guide to Robustness Analysis}

Robustness Analysis answers Question 2: How robust are model reponses (outputs) to local parameter perturbations?

(1) Decide which model parameters $\mathrm{p}^{1}, \mathrm{p}^{2}, . ., \mathrm{p}^{\mathrm{q}}$ to investigate. Then, for each $\mathrm{p}^{\mathrm{i},}$ decide which parameter perturbations $\mathrm{p}_{\mathrm{j}}^{\mathrm{i}}$ to investigate.

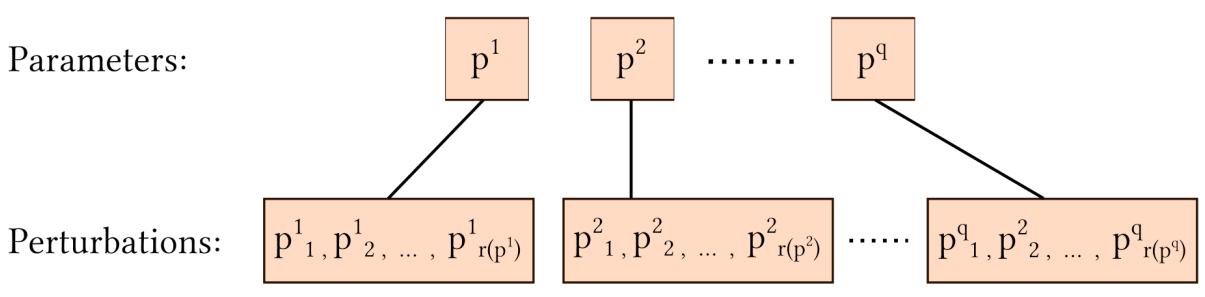

For each perturbation $\mathrm{p}_{\mathrm{j}}^{\mathrm{i}}$, produce a distribution $\mathrm{D}^{\prime}{ }_{\mathrm{n}^{*}}, \mathrm{p}_{\mathrm{j}}^{\mathrm{i}}$ containig $\mathrm{n}^{*}$ data samples resulting from in silico simulations produced using a set of model parameters in which (only) the parameter $p^{i}$ is perturbed (to the value $p_{j}^{i}$ ) and all other model parameters are kept at their calibrated value. 
(2) Using the $\hat{A}$-measure, compare all distributions $D_{n^{*}, p^{i j}}$ to the calibrated distribution $D_{n^{*}, p^{i}}$.

For each parameter $\mathrm{p}^{\mathrm{i}}$, plot these $\hat{\mathrm{A}}$-measures over the parameter value $\mathrm{p}_{\mathrm{j}}^{\mathrm{i}}$. These plots determine parameter ranges in which parameter perturbations yield a statistical significance that is small, medium or large (compared to the calibrated model parameters).

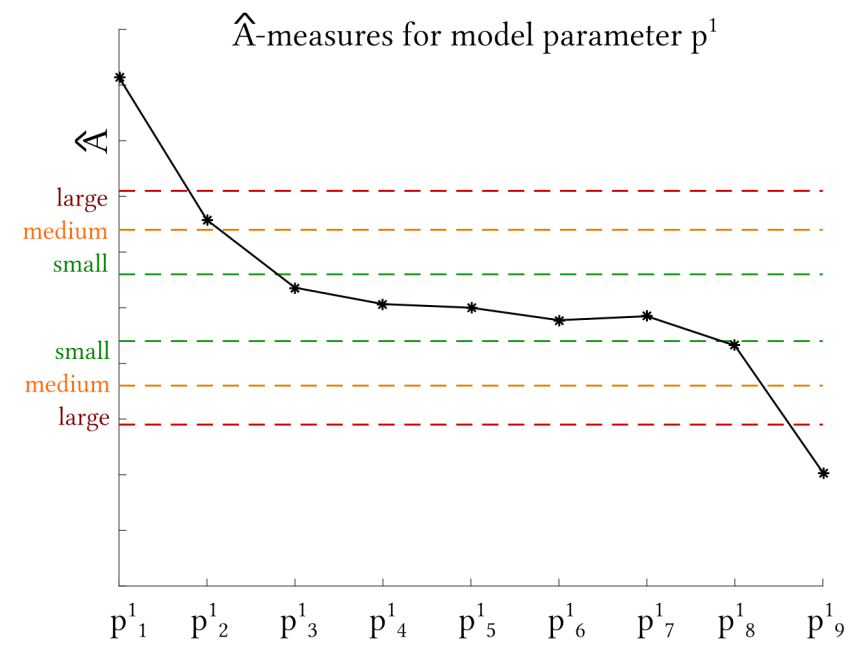

In the above plot, the statistical significance is small (within green lines) for the parameter value range $\left(\mathrm{p}_{3}^{1}, \mathrm{p}_{7}^{1}\right) \cdot \mathrm{p}_{5}^{1}$ is the calibrated value for $\mathrm{p}^{1 .}$ 
(3) For each parameter $p^{i}$, create boxplots over the perturbed value $p_{j}^{i}$. These plots graphically provide information regarding the influence that parameter value $\mathrm{p}_{\mathrm{i}}^{\mathrm{j}}$ has on the output $\mathrm{X}$.
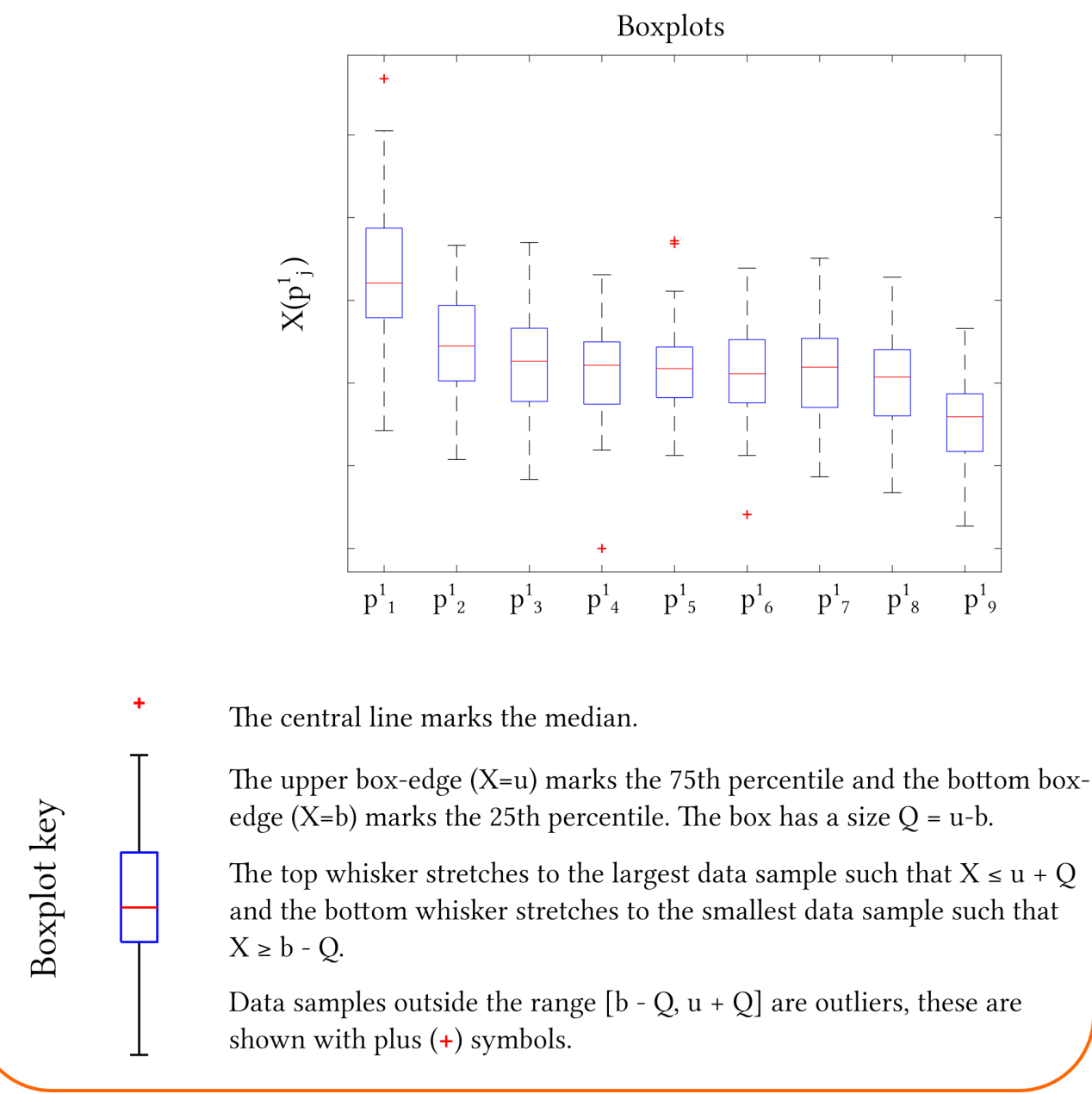

\section{A few remarks:}

- Robustness Analysis is a type of local sensitivity analysis.

- Robustness Analysis can be performed on one or multiple output variables X.

- When performing Robustness Analysis, model parameters are perturbed one at a time.

Figure 6.4: 3 -step quick guide on how to perform Robustness Analysis. 


\subsection{Latin Hypercube Sampling and Analysis}

Latin Hypercube Analysis answers the question: how robust are model responses to global parameter perturbations? Latin Hypercube Analysis is a type of global sensitivity analysis that investigates the relationship between input parameters and output responses when all input parameters are simultaneously perturbed. The parameters that we want to perturb are (as in Section 6.5) denoted $p^{i}$, where $i=1,2, . ., q$. Thus the parameters $p^{1}, p^{2}, \ldots, p^{q}$ together span a parameter space of dimension $q$. It is impossible to test every possible combination of input parameter values if they are picked from continuous ranges. In fact, even if we select a finite number of parameter values $r\left(p^{i}\right)$ to test for each parameter $p^{i}$, or if we pick discrete parameter values, comparing every possible combination of parameter values may require us to produce an impractically large number of simulation runs. Thus performing in silico simulations for all possible combinations of input parameters will in many cases be at worst impossible, and at best impractical. In order to circumvent this issue, Latin Hypercube Sampling can be used [64]. It is a sampling technique that ensures comprehensive testing coverage over the parameter space whilst keeping the number of tested parameter combinations low enough to be applicable in practice [217, 218]. After Latin Hypercube Sampling (Section 6.6.1), Latin Hypercube Analysis (Section 6.6.2 is used in order to assess global sensitivity.

\subsubsection{Latin Hypercube Sampling}

In the two-dimensional case, a Latin Square is an $\ell \times \ell$ square grid containing $\ell$ (traditionally Latin, hence the name) different symbols such that each symbol occurs exactly once in every row and exactly once in every column [219]. Analogously, in the Latin Hypercube Sampling framework, consider two parameters $p^{1}$ and $p^{2}$, spanning a parameter space of dimension $q=2$, where both $p^{1}$ and $p^{2}$ are sectioned into $\ell$ intervals. We then pick $\ell$ combinations of input parameter values (or sampling-points) $\left(p_{j}^{1}, p_{j}^{2}\right)$, where $j=1,2, \ldots, \ell$, such that every $p^{1}$-interval is sampled from exactly once and every $p^{2}$-interval is sampled from exactly once. Within the parameter range an interval, the sampled parameter value $p_{j}^{i}$ is randomly selected (unless of course the interval contains only one possible value $p_{j}^{i}$ ). Note that the $j$ index denotes the coordinate combination that $p_{j}^{i}$ belongs to, not the interval from which the parameter value $p_{j}^{i}$ was taken. Thus there is no condition demanding that the values $p_{j}^{i}$ are ordered in a way such that 


\section{UNCERTAINTY AND SENSITIVITY ANALYSES}

$p_{1}^{i}<p_{2}^{i}<\ldots<p_{\ell}^{i}$

The analogy between a Latin Square and Latin Hypercube Sampling from a twodimensional parameter space is illustrated in Figure 6.5. The Latin Square can be extended to higher dimensions to form a Latin Cube (dimension $=3$ ) or a Latin Hypercube (dimension $>3$ ) and, analogously, the two-dimensional sampling space illustrated in Figure 6.5 can be extended to $q$ dimensions, spanned by the input parameters $p^{1}, p^{2}, . ., p^{q}[219$.
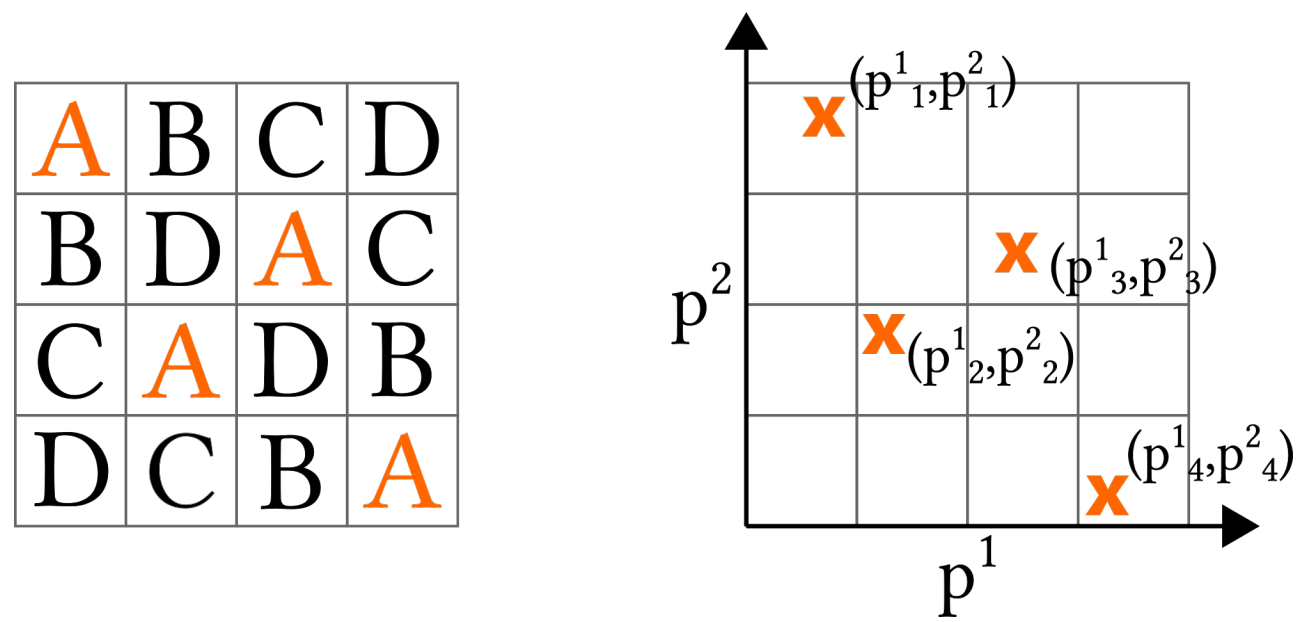

Figure 6.5: Left: An $\ell \times \ell$ Latin Square in which each Latin symbol occurs $\ell$ times, exactly once in each row and exactly once in each column. Right (analogously): A two-dimensional parameter space spanned by the input parameters $p^{1}$ and $p^{2}$ that are both sectioned into $\ell$ intervals. Using Latin Hypercube sampling, $\ell$ parameter combinations $\left(p_{j}^{1}, p_{j}^{2}\right)$ are sampled where $j=1,2, . ., \ell$ and each $p^{1}$-interval is sampled from exactly once and each $p^{2}$-interval is sampled from exactly once.

For each parameter $p^{i}$, the total investigated parameter range is $\left[\min \left(p^{i}\right), \max \left(p^{i}\right)\right]$, where $\min \left(p^{i}\right)$ and $\max \left(p^{i}\right)$ respectively denote the minimum and maximum values of $p^{i}$ to be investigated. Now each parameter range $\left[\min \left(p^{i}\right), \max \left(p^{i}\right)\right]$ is sectioned into $N$ intervals, and we denote these intervals by $u_{p^{i}}^{1}, u_{p^{i}}^{2}, \ldots ., u_{p^{i}}^{N}$. Note that, all input parameters $p^{i}$ must be sectioned into the same number of intervals. If the intervals are uniformly distributed, then the size of an interval, $w\left(p^{i}\right)$, is

$$
w\left(p^{i}\right)=\frac{\max \left(p^{i}\right)-\min \left(p^{i}\right)}{N}
$$


and the $r$-th interval $u_{p^{i}}^{r}$ has a parameter range such that

$$
u_{p^{i}}^{r}=\left[\min \left(p^{i}\right)+w \cdot(r-1), \min \left(p^{i}\right)+w \cdot r\right]
$$

where $r=1,2, \ldots, N$.

Note that there are more than one way to populate Latin symbols in a Latin Square, this can be realised by regarding Figure 6.5 and noticing that the A-symbols and the $\mathbf{B}$ symbols cover the Latin Square in different ways. Analogously, and by extension, there are multiples ways to populate sampling coordinates in a Latin Hypercube Sampling framework. Some of these ways provide better coverage of the parameter space than do others [219], but details regarding such sampling-optimisation are outside the scope of this study. In this study, we use the built-in MATLAB function Ihsdesign [205] to select which parameter combinations to use according to a Latin Hypercube Sampling approach. Note that, in our case, all $N$ intervals $u_{p^{i}}^{1}, u_{p^{i}}^{2}, \ldots ., u_{p^{i}}^{N}$ for a parameter $p^{i}$ are uniformly spaced, but the choice of spacing can be adjusted to the specific application at hand [205].

Now let us address the choice of intervals $N$, as this is not straightforward. Using the Latin Hypercube Sampling framework, every parameter $p^{i}$, where $i=1,2, . ., q$, is partitioned into $N$ intervals and, consequently, $N$ combinations comprising $q$ parameter values are sampled and tested. Compared to a small $N$-value, a large value of $N$ will provide more data to use, and draw conclusions from, in the Latin Hypercube Analysis stage, however, it will also increase the computational cost in the Latin Hypercube Sampling stage. There is no strict rule for how to choose $N$, but suggested values for $N$ in the literature are $N=2 q$ for large values of $q$ (i.e. high-dimensional parameter spaces) or $N=4 q / 3$ which has been described to be 'usually satisfactory' [220, 221]. Authors of the SPARTAN package use a lot larger numbers in their provided examples [64]. In this study, we decide to use $N=100$ uniform intervals. (We tried using only 10 intervals, in accordance with the suggestion $N=10>4 q / 3 \approx 9$, but this did not enable us to distinguish any trends in the data in the Analysis stage. However with $N=100$, we had sufficient data samples from which to draw conclusions from in the Analysis stage, as demonstrated in Section 6.6.3. At the end of the day, the choice of $N$ is up to the modeller, who must outweigh the (computational) cost of producing a 


\section{UNCERTAINTY AND SENSITIVITY ANALYSES}

large number of data samples, with the advantage of having a vast amount of data, and thus plentiful information, in the analysis stage. Quantitative choices of $N$ are outside the scope of this study.

\subsubsection{Latin Hypercube Analysis}

During the Latin Hypercube Sampling process, $N$ different points in the $q$-dimensional parameter space spanned by the input parameters $p^{1}, p^{2}, \ldots, p^{q}$ are selected as samplingpoints, as shown in Step 1 in Section 6.6.3. One such sampling-point, $C_{j}$, can be described by its coordinates in the parameter space so that $C_{j}=\left(p_{j}^{1}, p_{j}^{2}, \ldots, p_{j}^{q}\right)$. Each sampling-point $C_{j}$ is used to generate $n^{*}$ output values $X\left(C_{j}\right)$, where $n^{*}$ is determined using Consistency Analysis. Subsequently, the median output value, here denoted $\underset{\sim}{X}\left(C_{j}\right)$, is computed for every $C_{j}$. Now, our overall aim is to investigate the relationship between an input parameter $p^{i}$ and an output response $X$. We investigate this input-output relationship in two steps, one of which is qualitative and one of which is quantitative. In the first, and qualitative, step we produce two-dimensional scatterplots in which median output data,

$$
\begin{aligned}
& \underset{\sim}{X}\left(C_{1}\right), \underset{\sim}{X}\left(C_{2}\right), \ldots, \underset{\sim}{X}\left(C_{N}\right)=\underset{\sim}{X}\left(p_{1}^{1}, p_{1}^{2}, \ldots, p_{1}^{q}\right), \underset{\sim}{X}\left(p_{2}^{1}, p_{2}^{2}, \ldots, p_{2}^{q}\right), \ldots, \underset{\sim}{X}\left(p_{N}^{1}, p_{N}^{2}, \ldots, p_{N}^{q}\right), \\
& \text { are plotted over parameter values }
\end{aligned}
$$

$$
p_{1}^{i}, p_{2}^{i}, \ldots, p_{N}^{i},
$$

for one of the input parameters $p^{i}$. We do this for every input parameter $i=1,2, \ldots, q$ and thus $q$ scatterplots are created. By simply visually analysing the data in the scatterplots, we are able to make qualitative observations regarding the relationship between the input and the parameter. Examples of such observations are provided in Step 2 in Section 6.6 .3

As a second step, we use a quantitative measure, such as the Pearson Product Moment Correlation Coefficient (or the correlation coefficient for short), to quantitatively describe the correlation between input parameters and output responses, as done in Step 3 in Section 6.6.3. The correlation coefficient is denoted $r$, where $r \in[-1,+1]$, describes the linear association between the input parameter and the output response in 
terms of both magnitude and direction. A positive (linear) correlation between $p^{i}$ and $\tilde{X}\left(C_{j}\right)$ means that if either the input value or the output value increases, so does the other one, and thus $r$ is positive. Conversely, a negative correlation means that if either $p^{i}$ or $\tilde{X}\left(C_{j}\right)$ increases, the other one decreases, and thus $r$ is negative. The magnitude of $r$ describes the strength of the correlation, where a magnitude of 1 corresponds to a strong linear association, and a small magnitude corresponds to a weak correlation. An $r$-value of approximately zero indicates that there is no linear correlation between the two investigated variables. Note that the Pearson Product Moment Correlation Coefficient picks up linear associations only, thus there may exist other, non-linear correlations that are not captured by the correlation coefficient $r$. Therefore it is important to, not only quantitatively compute input-output correlations, but to also qualitatively assess the relationships between inputs and outputs, via data visualisation in scatterplots as previously described 1

The correlation coefficient, $r^{i}$, describing the correlation between an input parameter $p^{i}$, and an output response $X$ is given by [222],

$$
r^{i}=\frac{\sum_{j=1}^{N}\left(p_{j}^{i}-\bar{p}^{i}\right)\left(\underset{\sim}{X}\left(C_{j}\right)-\underset{\sim}{\bar{X}}\right)}{\sqrt{\left(\sum_{j=1}^{N}\left(p_{j}^{i}-\bar{p}^{i}\right)^{2}\right)\left(\sum_{j=1}^{N}\left(\underset{\sim}{X}\left(C_{j}\right)-\underset{\sim}{X}\right)^{2}\right)}} .
$$

The correlation coefficient $\mathrm{r}$ picks up on linear relationships only, thus the scatterplot to the left yields $r=0$. (As an intuitive example, let $\boldsymbol{x}$ represent 'statements certain politicians make' and $y$ represent 'the truth of these statements'). 


\section{UNCERTAINTY AND SENSITIVITY ANALYSES}

When it comes to interpreting quantitative input-output relationships based on the correlation coefficient $r$, there are no all-encompassing threshold values to use for descriptors such as 'weak', 'moderate', 'strong' [222 224]. Relationships quantified by correlation coefficient values close to the extrema 0 or 1 may be easy to describe as 'negligible' or 'strong', respectively but correlation coefficient values in the middle of the $[0,1]$ range are more difficult to label. Various 'rule of thumbs' have been suggested in the literature but, at the end of the day, it is up to the researcher to appropriately judge what constitutes a 'weak', 'moderate' or 'strong' input-output relationship in the specific (modelling) application at hand, taking into account the research area, the number of data samples, and the range of investigated input values [223]. However, even without rigid descriptor threshold values, we can compare the correlation coefficient values for all input-output pairs and see which input values are the most influential within the ranges of regarded input values. As a guide, suggested correlation coefficient descriptor threshold values presented in the literature are listed in Table 6.1. The methodology to perform Latin Hypercube Sampling and Analysis is outlined in Section 6.6 .3

\begin{tabular}{|c||c|c|c|c|c|}
\hline & $\begin{array}{c}\text { descriptor } \\
\text { negligi- } \\
\text { ble }\end{array}$ & weak & $\begin{array}{c}\text { moder- } \\
\text { ate }\end{array}$ & strong & $\begin{array}{c}\text { very } \\
\text { strong }\end{array}$ \\
\hline Mukaka [222] & {$[0,0.3)$} & {$[0.3,0.5)$} & {$[0.5,0.7)$} & {$[0.7,0.9)$} & {$[0.9,1]$} \\
\hline Schober et al. $[223]$ & {$[0,0.1)$} & {$[0.1,0.4)$} & {$[0.4,0.7)$} & {$[0.7,0.9)$} & {$[0.9,1]$} \\
\hline Krehbiel [224] & "A linear relationship exists if $|r| \geq 2 / \sqrt{\text { number of samples." }}$ \\
\hline \hline
\end{tabular}

Table 6.1: Suggested descriptor threshold values for the magnitude of the correlation coefficient, $|r|$, reported in the literature.

\subsubsection{Quick Guide to Latin Hypercube Sampling and Analysis}

Latin Hypercube Analysis answers Question 3: How sensitive are model reponses (outputs) to global parameter perturbations? 
1 Decide which model parameters $\mathrm{p}^{1}, \mathrm{p}^{2}, \ldots, \mathrm{p}^{\mathrm{q}}$ to investigate. Then, for each $\mathrm{p}^{\mathrm{i}}$ decide which parameter range $\left[\min \left(\mathrm{p}^{\mathrm{i}}\right), \max \left(\mathrm{p}^{\mathrm{i}}\right)\right]$ to investigate. Split every such parameter range into $N$ intervals $u_{p^{1}}^{1}, u_{p^{i}}^{2}, \ldots, u_{p^{1}}^{N}$.

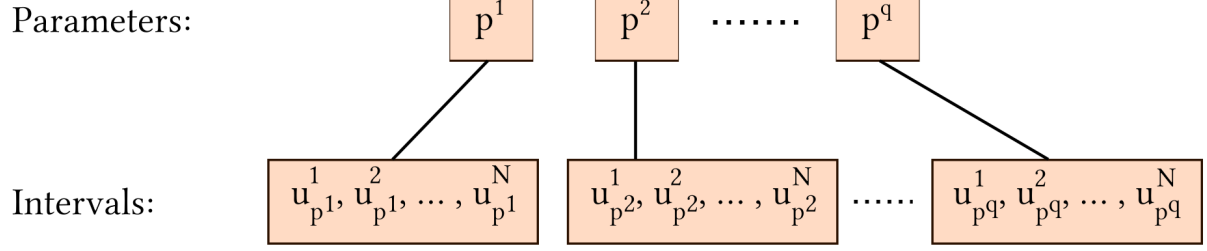

Using Latin Hypercube Sapmling, select $N$ sampling points $C_{j}=\left(p_{j}^{1}, p_{j}^{2}, \ldots, p_{j}^{q}\right)$ such that every interval is sampled from exactly once. 
2 For every sampling point $C_{j}$, perform $n^{*}$ in silico runs (where $n^{*}$ is chosen 2 via consistency analysis) and compute the median output response $\underset{\sim}{\mathrm{X}}\left(\mathrm{C}_{\mathrm{j}}\right)$.

For every investigated input parameter $\mathrm{p}^{\mathrm{i}}$, create a scatterplot that plots the median output values

$$
\underset{\sim}{\mathrm{X}}\left(\mathrm{C}_{1}\right), \underset{\sim}{\mathrm{X}}\left(\mathrm{C}_{2}\right), \ldots, \underset{\sim}{\mathrm{X}}\left(\mathrm{C}_{\mathrm{N}}\right)
$$

over their repective input parameter values

$$
\mathrm{p}_{1}^{\mathrm{i}}, \mathrm{p}_{2}^{\mathrm{i}}, \ldots, \mathrm{p}_{\mathrm{N}}^{\mathrm{i}}
$$

Observe the data, visualised by scatter-plots, to draw qualitative conclusions about the input-output relationships.
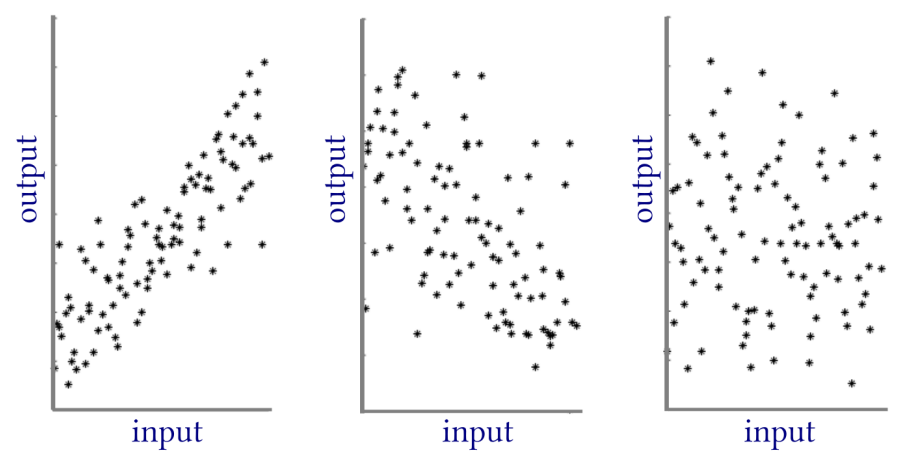

Qualitative

The input and output are strongly, positiveremarks: ly correlated.

The input and output The input and output are moderately, neg- are not correlated. atively correlated. 
3 For every investigated input parameter $\mathrm{p}^{\mathrm{i}}$, assess the (linear) quantitative correlation between the median output values

$$
\underset{\sim}{\mathrm{X}}\left(\mathrm{C}_{1}\right), \underset{\sim}{\mathrm{X}}\left(\mathrm{C}_{2}\right), \ldots, \underset{\sim}{\mathrm{X}}\left(\mathrm{C}_{\mathrm{N}}\right)
$$

and the input parameter values

$$
\mathrm{p}_{1}^{\mathrm{i}}, \mathrm{p}_{2}^{\mathrm{i}}, \ldots, \mathrm{p}_{\mathrm{N}}^{\mathrm{i}}
$$

using the correlation coefficient $\mathrm{r}$.
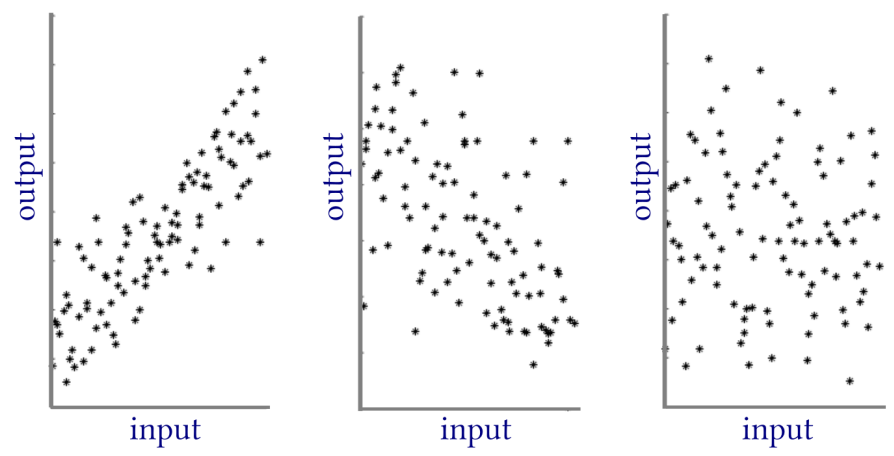

\section{Quantitative} measure:

$$
\mathrm{r}=0.84
$$

$r=-0.59$

$r=-0.02$

\section{A few remarks:}

- Latin Hypercube Analysis is a type of global sensitivity analysis.

- Latin Hypercube can be performed on one or multiple output variables X.

- When performing Robustness Analysis, all model parameters are simultaneously perturbed.

Figure 6.6: 3 -step quick guide on how to perform Latin Hypercube Sampling and Analysis.

\subsection{Case Study: Analysing the Results from Chapter 5}

To evaluate the in silico findings obtained in the in vitro study described in Chapter 5 , three uncertainty and sensitivity analyses techniques are performed. The three techniques are namely: (1) Consistency Analysis, which is used to determine how many in 


\section{UNCERTAINTY AND SENSITIVITY ANALYSES}

silico runs should be performed before defining results in terms of statistical metrics in order to mitigate uncertainty originating from intrinsic model stochasticity, (2) Robustness Analysis, which investigates model sensitivity to local parameter perturbations and (3) Latin Hypercube Analysis, which investigates model sensitivity to global parameter perturbations. To perform uncertainty and sensitivity analyses we need to specify a set of inputs and outputs. Here, the output variables are $X_{1}$ : the percentage of $\gamma \mathrm{H} 2 \mathrm{AX}-$ positive (i.e. damaged) cells at the end time of the experiment (72 hours), and $X_{2}$ : the cell count (i.e. the number of non-dead cells) at the end of the experiment. The input variables are the seven model parameters listed in Table 5.1 that we calibrate using in vitro data. These inputs are namely $\mu, \sigma, \Pi_{D-S}, \Theta_{D-S}, E C_{50}, \gamma$ and $T_{L \rightarrow D}$.

\subsubsection{Consistency Analysis}

Results from the Consistency Analysis are provided in Figures 6.7, 6.8, 6.9, 6.10, 6.11 which show the $\hat{A}$-measures, in both computed and scaled forms, for the distribution sizes $n=1,5,50,100,300$ respectively. By observing Figures 6.7 through to 6.11 , it is clear that the statistical significance decreases with increasing distribution size $n$, as is shown in Figure 6.12 and Table 6.2 which show the maximal scaled $\hat{A}$-values for all tested distribution sizes. These results demonstrate that the distribution size $n=100$ is the smallest tested distribution size that yields a small statistical significance (i.e. a maximum scaled $\hat{A}$-value smaller than 0.56 ) for both regarded output variables $X_{1}$ and $X_{2}$. From this we decide to base every in silico result (here in terms of mean values and standard deviations) on 100 simulation runs.

\begin{tabular}{|c||c|c|c|c|c|}
\hline $\begin{array}{r}\text { distribution } \\
\text { size }\end{array}$ & $\mathrm{n}=1$ & $\mathrm{n}=5$ & $\mathrm{n}=50$ & $\mathrm{n}=100$ & $\mathrm{n}=300$ \\
\hline$X_{1}$ & & & & & \\
\hline$X_{2}$ & 1 & 0.92 & 0.61 & 0.55 & 0.54 \\
\hline
\end{tabular}

Table 6.2: Maximal scaled $\hat{A}$-values produced in the Consistency Analysis for various distribution sixes $n$. The output variables are $X_{1}$, corresponding to the percentage of $\gamma \mathrm{H} 2 \mathrm{AX}$ positive (i.e. damaged) cells, and $X_{2}$, corresponding to the cell count. 


\subsubsection{Robustness Analysis}

We use Robustness Analysis to investigate how sensitive the output is to local parameter perturbations, that is to say when input parameters are varied one at a time. Figures $6.13,6.14,6.15,6.16,6.17,6.18,6.19$ provide boxplots and $\hat{A}$-measures that demonstrate the effect that local perturbations of the input variables $\mu, \sigma, \Pi_{D-s}, \Theta_{D-S}, E C_{50}, \gamma$ and $T_{L \rightarrow D}$ respectively have on the output variables $X_{1}$ and $X_{2}$. Key findings are listed below, discussing the impact of one input parameter at a time.

- Remarks regarding input parameter $\mu$ : Figure 6.13 shows that, for small parameter perturbations, increasing the average doubling times of cells, $\mu$, overall decreases the percentage of $\gamma \mathrm{H} 2 \mathrm{AX}$ positive cells and increases the cell count, however this decrease/increase is not linear. This indicates that the results of the in vitro simulation (and of the in vitro experiment nonetheless) are sensitive to the timing of the drug administration. In other words, Robustness Analyses demonstrates that treatment responses depend on how many cells are in the susceptible cell-cycle state at time of drug administration.

- Remarks regarding input parameter $\sigma$ : Figure 6.14 demonstrates that the level of cell cycle synchronisation amongst cells, quantified by the input $\sigma$, affects in silico outputs for small parameter perturbations. The results indicate that for highly asynchronised cells (i.e. high $\sigma$-values) the smoother growth curves yield higher cell counts at certain time-points (such as the end time 72 hours) and a lower percentage of $\gamma \mathrm{H} 2 \mathrm{AX}$-positive cells. As discussed in the remark above, the timing between cell cycles and drug administration affect treatment responses.

- Remarks regarding input parameter $\Pi_{D-S}$ : Figure 6.15illustrates that increasing the probability that a cell enters the damaged $S$ state, i.e. the variable $\Pi_{D-S}$, increases the percentage of $\gamma \mathrm{H} 2 \mathrm{AX}$ cells and decreases the cell count, as expected.

- Remarks regarding input parameter $\Theta_{D-S}$ : Figure 6.16 shows how the amount of time that damaged cells spend in the D-S state before attempting to repair, and thus the $\Theta_{D-S^{-}}$value, affects the output. Results show that the percentage of $\gamma \mathrm{H} 2 \mathrm{AX}$ positive cells increases with increasing values of $\Theta_{D-S}$, as more damaged cells will accumulate in the D-S state. However, this does not affect the probability of cells repairing, so the cell count is not as sensitive to small perturbations of 


\section{UNCERTAINTY AND SENSITIVITY ANALYSES}

$\Theta_{D-S}$. The value of $\Theta D-S$ implicitly affects the measured cell count at the end time of the experiment as a decreased/increased $\Theta D-S$-value yields a slightly decreased/increased time lag between a cell entering the D-S state and dying.

- Remarks regarding input parameter $E C_{50}$ : Figure 6.17demonstrates that output variables are highly sensitive to perturbations of $E C_{50}$. Increasing $E C_{50}$ results in a higher percentage of $\gamma \mathrm{H} 2 \mathrm{AX}$ positive cells and a lower cell count. Thus the input parameter $E C_{50}$ should be regarded as a highly influential on quantitative results.

- Remarks regarding input parameter $\gamma$ : Figure 6.18 illustrates that output variables measured at the end time of the experiment are not very sensitive to small perturbations of $\gamma$. This can be understood as the $\gamma$ parameter inherently corresponds to 'how quickly' a drug achieves asymptotic behaviour in the $E_{\max }$ model, the model used in our mathematical framework to express cellular drug response.

- Remarks regarding input parameter $T_{L \rightarrow}$ : Figure6.19 shows how output variables change as a result of perturbations to the input variable $T_{L \rightarrow}$, that describes how long it takes for a cell that has failed to repair to die (i.e. how long a cell with a 'death-sentence' is picked up as $\gamma \mathrm{H} 2 \mathrm{AX}$ positive in the in vitro experiment). Results show that both the percentage of $\gamma \mathrm{H} 2 \mathrm{AX}$ positive cells and the cell count increases with increasing values of $T_{L \rightarrow}$, as dying cells will categorised as $\gamma \mathrm{H} 2 \mathrm{AX}$ positive longer before being categorised as dead. When calibrating the model, we avoid the effect of this input parameter by only regarding in vitro data at time points that are early enough to correspond to systems with no (or a negligible amount of) dead cells.

\subsubsection{Latin Hypercube Analysis}

Latin Hypercube Analysis is here used to investigate how sensitive output responses are to global parameter perturbations. We here investigate parameter values within ranges that we consider to be 'plausible' from the calibration process and the Robustness Analysis. Figures 6.20, 6.21, 6.22, 6.23, 6.24, 6.25, 6.26 provide scatter-plots that demonstrate correlations between the output variables $X_{1}$ and $X_{2}$ and the input variables $\mu, \sigma, \Pi_{D-s}, \Theta_{D-S}, E C_{50}, \gamma$ and $T_{L \rightarrow D}$ respectively. The Pearson Product Moment Correlation Coefficients between the various input-output pairs are listed in Table 6.3 . 
To determine threshold values for correlation coefficient descriptors, we compromise between suggested values by other authors [204, and take into account the fact that we are only regarding parameter values within 'plausible' ranges. With this as a guide, we here decide that our obtained correlation coefficients with a magnitude in $[0,0.12]$ corresponds to the linear input-output relationship being 'negligible', [0.19, 0.35] 'weak', [0.48,0.59] 'moderate' and 0.84 'strong'. Key findings from the Latin Hypercube Analysis are listed below, where the impact of one input parameter is discussed one at a time.

- Remarks regarding input parameter $\mu$ : Figure 6.20 and the first column in Table 6.3 show that, for the allowed parameter range, $\mu$ and $X_{1}$ are moderately, negatively correlated as the correlation coefficient is -0.48 and the scatterplot displays an overall trend of the output $\left(X_{1}\right)$ decreasing with increasing values of the input $\mu$. The relationship between $\mu$ and the other output variable $X_{2}$ is, on the other hand, negligible. We explain this by the fact that treatment responses are sensitive to the timing of the drug administration, but there is a time-lag $T_{L \rightarrow D}$ between a cell's lethal event (failure to repair) and its death. As damaged (but not dead) cells are included in the cell count, the $\left(\mu, X_{2}\right)$-relationship is more weakly linearly correlated than the $\left(\mu, X_{1}\right)$-relationship.

- Remarks regarding input parameter $\sigma$ : Figure 6.21 and the second column in Table 6.3 demonstrate that the linear relationships between input variable $\sigma$ and the output variables $X_{1}$ and $X_{2}$ are both negligible, within the regarded input parameter value range.

- Remarks regarding input parameter $\Pi_{D-S}$ : Figure 6.22 and the third column in Table 6.3 indicate that the relationships between the input variable $\Pi_{D-S}$ and the output variables $X_{1}$ and $X_{2}$ are, respectively, positively and negatively weakly linearly correlated. This agrees with the intuitive notion that if the probability that a cell enters the D-S state increases, cell damage $\left(X_{1}\right)$ increases whilst the cell count $\left(X_{2}\right)$ decreases.

- Remarks regarding input parameter $\Theta_{D-S}$ : Figure 6.23 and the fourth column in Table 6.3 show that the input variable $\Theta_{D-S}$ is has a negligible linear correlation with the output variables $X_{1}$ and $X_{2}$. 


\section{UNCERTAINTY AND SENSITIVITY ANALYSES}

- Remarks regarding input parameter $E C_{50}$ : Figure 6.24 and the fifth column in Table 6.3 demonstrate that the input variable $E C_{50}$ impacts the output responses more than do other input variables, within the regarded ranges for input variables. $E C_{50}$ is negatively, moderately linearly correlated with $X_{1}$ and $E C_{50}$ is strongly, positively linearly correlated with $X_{2}$. These relationships are visually apparent in the regarded scatterplots.

- Remarks regarding input parameter $\gamma$ : Figure 6.25 and the sixth column in Table 6.3 indicate negligible linear correlations between the input parameter $\gamma$ and both output variables $X_{1}$ and $X_{2}$.

- Remarks regarding input parameter $T_{L \rightarrow D}$ : Figure 6.26 and the last column in Table 6.3 demonstrate that the input variable $T_{L \rightarrow D}$ is positively, weakly, linearly correlated with the output $X_{1}$, whilst the linear correlation between $T_{L \rightarrow D}$ and $X_{2}$ is negligible.

\begin{tabular}{|c||c|c|c|c|c|c|c|}
\hline & $\mu$ & $\sigma$ & $\Pi_{D-S}$ & $\Theta_{D-S}$ & $E C_{50}$ & $\gamma$ & $T_{L \rightarrow D}$ \\
\hline$X_{1}$ & & & & & & & \\
\hline$X_{2}$ & 0.12 & 0.06 & 0.19 & 0.06 & -0.59 & 0.05 & 0.35 \\
\hline
\end{tabular}

Table 6.3: Pearson Product Moment Correlation Coefficients between input and output variables obtained in the Latin Hypercube Analysis. 

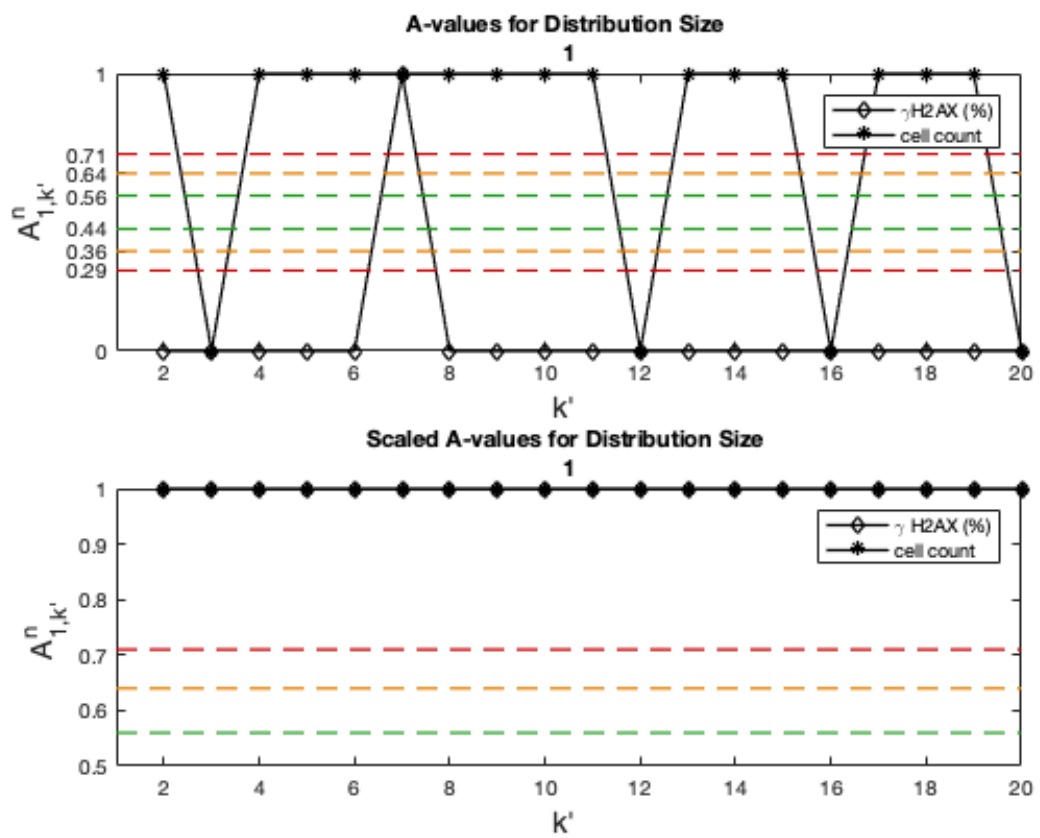

Figure 6.7: Consistency Analysis. $\hat{A}$-values in initial (top) and scaled (bottom) form for distribution size $n=1$.

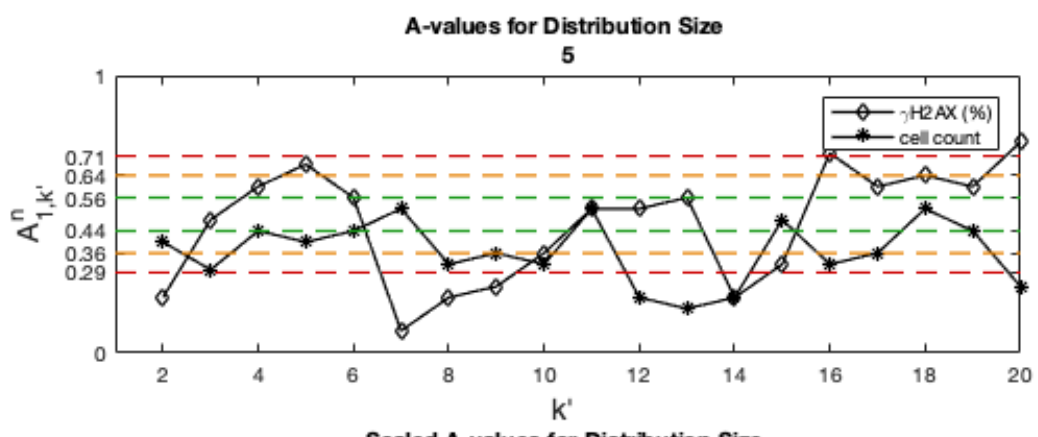

Scaled A-values for Distribution Size

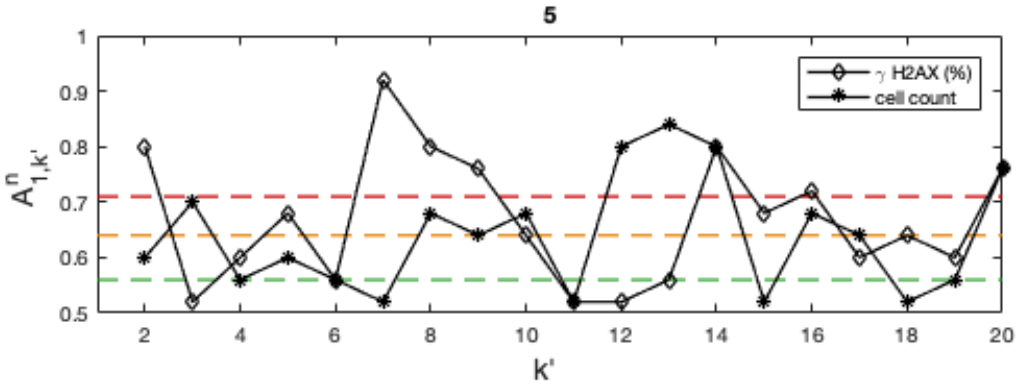

Figure 6.8: Consistency Analysis. $\hat{A}$-values in initial (top) and scaled (bottom) form for distribution size $n=5$. 


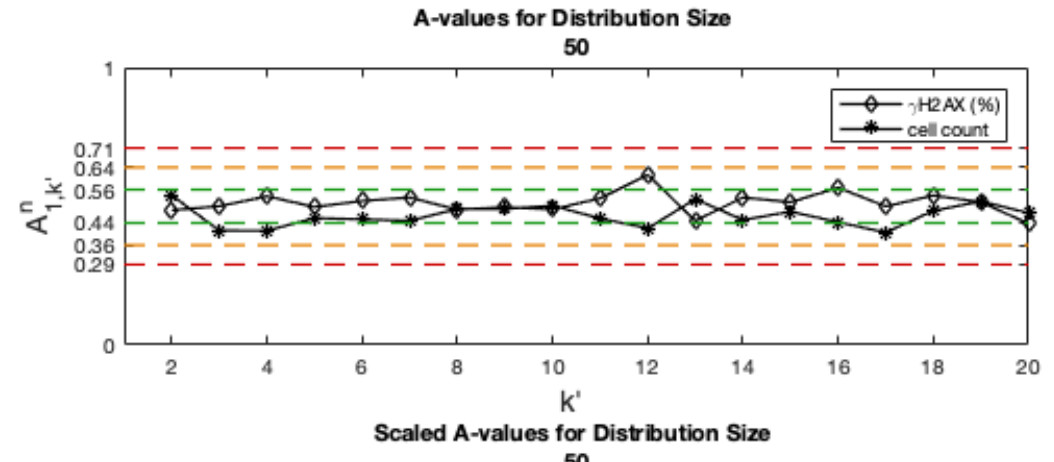

50

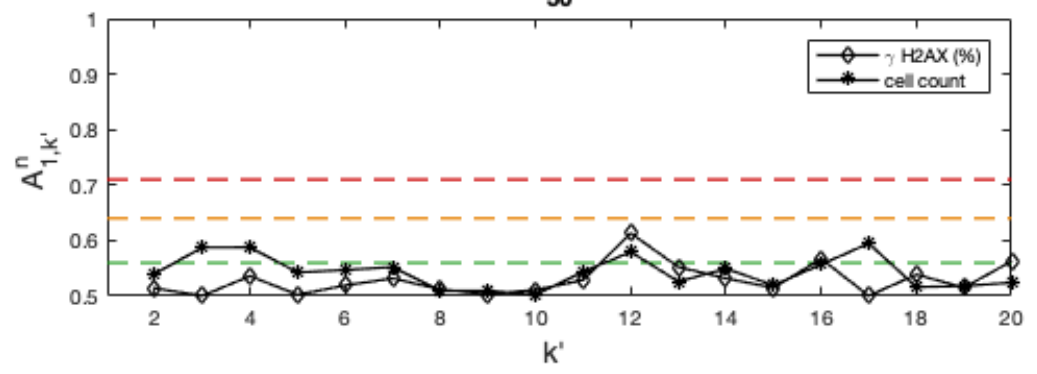

Figure 6.9: Consistency Analysis. $\hat{A}$-values in initial (top) and scaled (bottom) form for distribution size $n=50$.
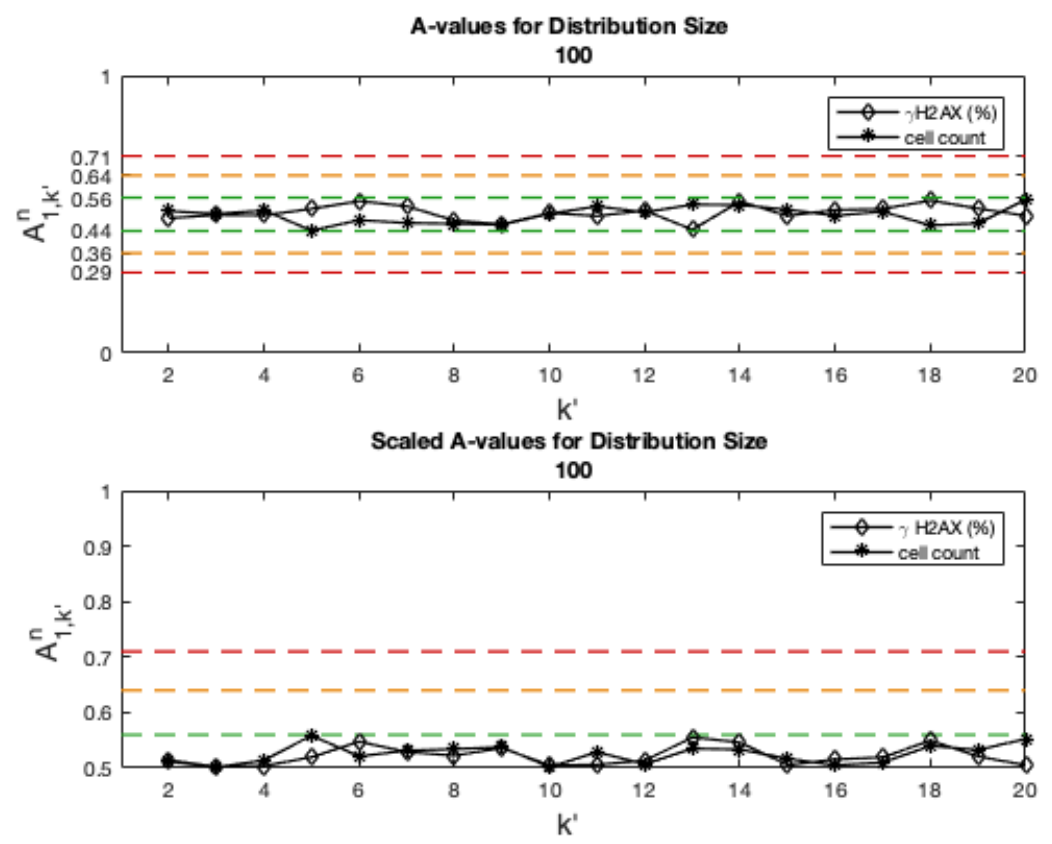

Figure 6.10: Consistency Analysis. $\hat{A}$-values in initial (top) and scaled (bottom) form for distribution size $n=100$. 


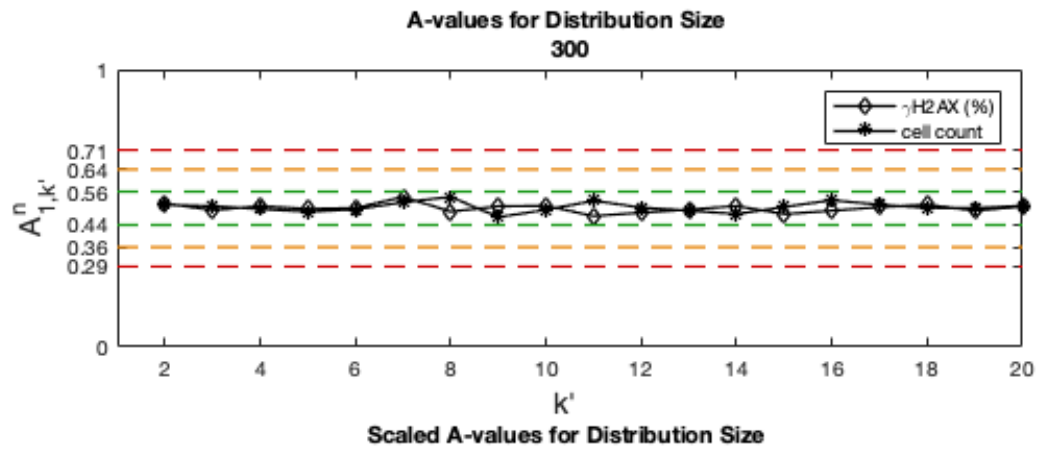

300

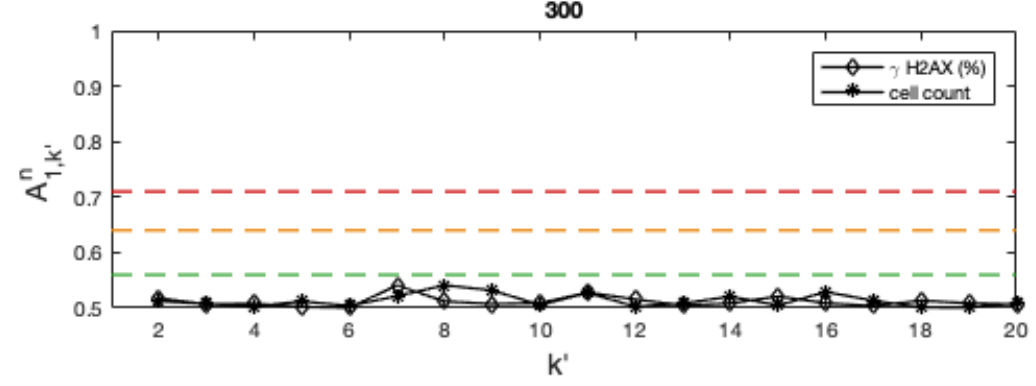

Figure 6.11: Consistency Analysis. $\hat{A}$-values in initial (top) and scaled (bottom) form for distribution size $n=300$.

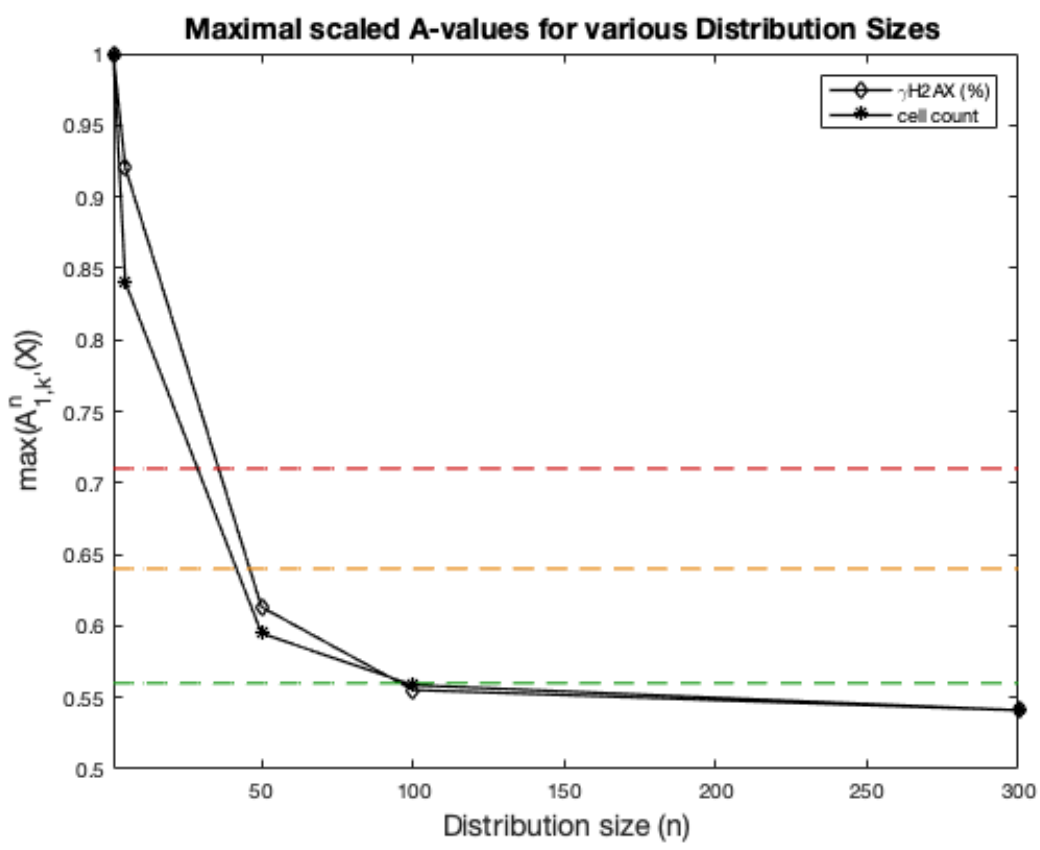

Figure 6.12: Consistency Analysis. Scaled $\hat{A}$-values for various distribution sizes tested in the Consistency Analysis. 
Output responses to perturbations of $\mu$
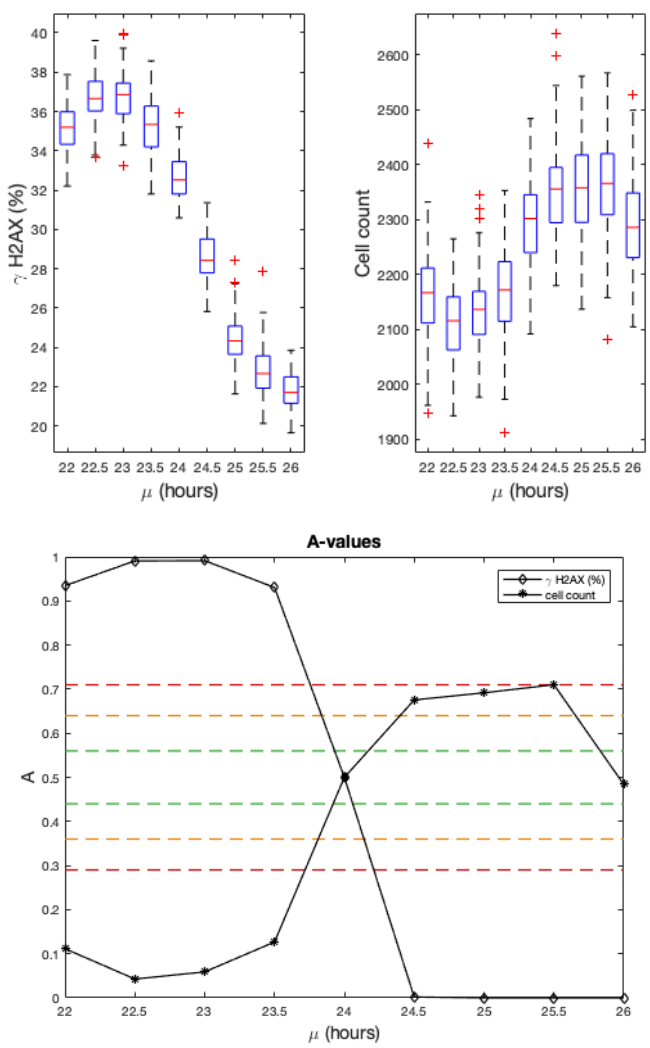

Figure 6.13: Robustness Analysis. Left: Output responses, in terms of percentage of $\gamma \mathrm{H} 2 \mathrm{AX}$ positive (i.e. damaged) cells, and cell count as a result of perturbations to the input variable $\mu$. Right: Maximal $\hat{A}$-values resulting from comparisons between distributions with perturbed data and a distribution with calibrated (unperturbed) data. 
Output responses to perturbations of $\sigma$
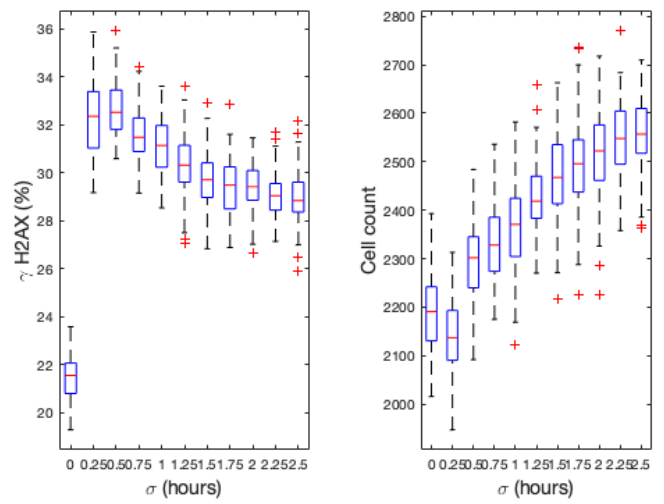

A-values

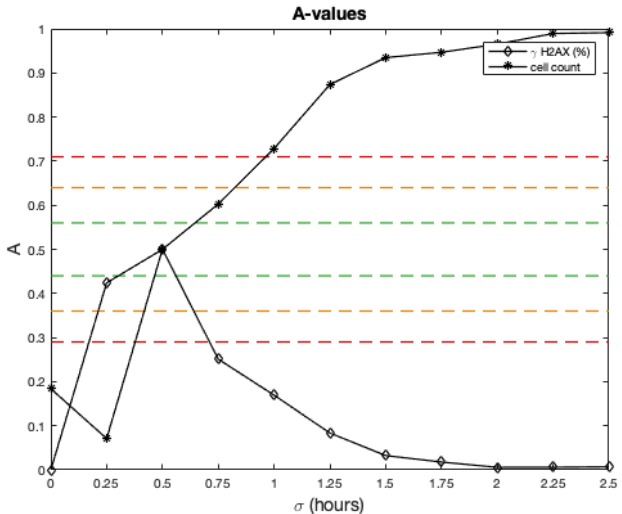

Figure 6.14: Robustness Analysis. Left: Output responses, in terms of percentage of $\gamma \mathrm{H} 2 \mathrm{AX}$ positive (i.e. damaged) cells, and cell count as a result of perturbations to the input variable $\sigma$. Right: Maximal $\hat{A}$-values resulting from comparisons between distributions with perturbed data and a distribution with calibrated (unperturbed) data. 

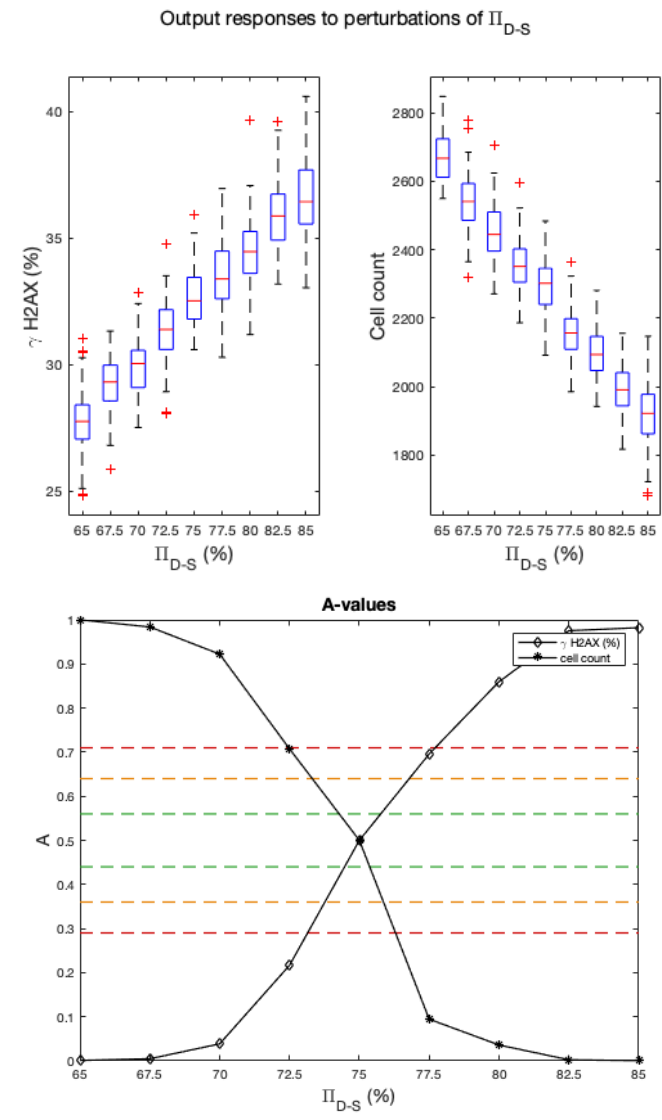

Figure 6.15: Robustness Analysis. Left: Output responses, in terms of percentage of $\gamma \mathrm{H} 2 \mathrm{AX}$ positive (i.e. damaged) cells, and cell count as a result of perturbations to the input variable $\Pi_{D-s}$. Right: Maximal $\hat{A}$-values resulting from comparisons between distributions with perturbed data and a distribution with calibrated (unperturbed) data. 
Output responses to perturbations of $\Theta_{D-S}$
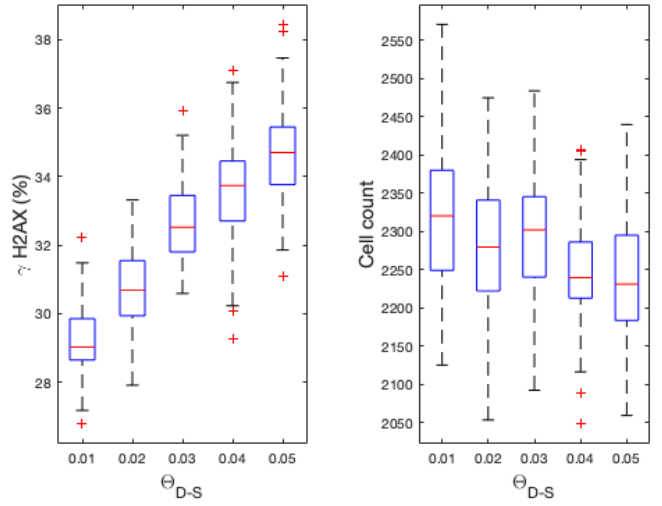

A-values

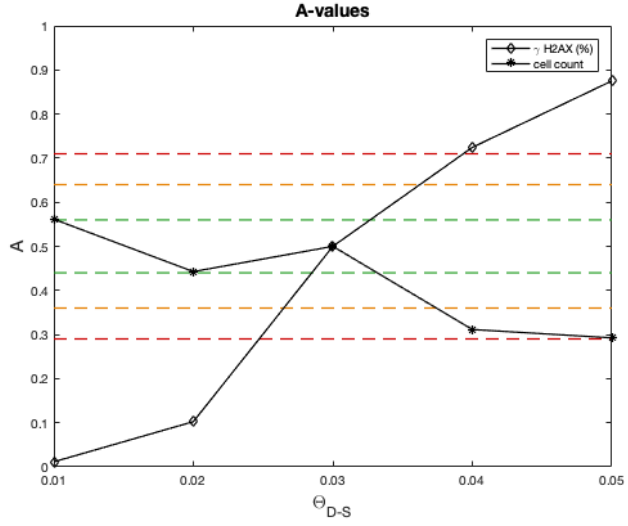

Figure 6.16: Robustness Analysis. Left: Output responses, in terms of percentage of $\gamma \mathrm{H} 2 \mathrm{AX}$ positive (i.e. damaged) cells, and cell count as a result of perturbations to the input variable $\Theta_{D-S}$. Right: Maximal $\hat{A}$-values resulting from comparisons between distributions with perturbed data and a distribution with calibrated (unperturbed) data. 

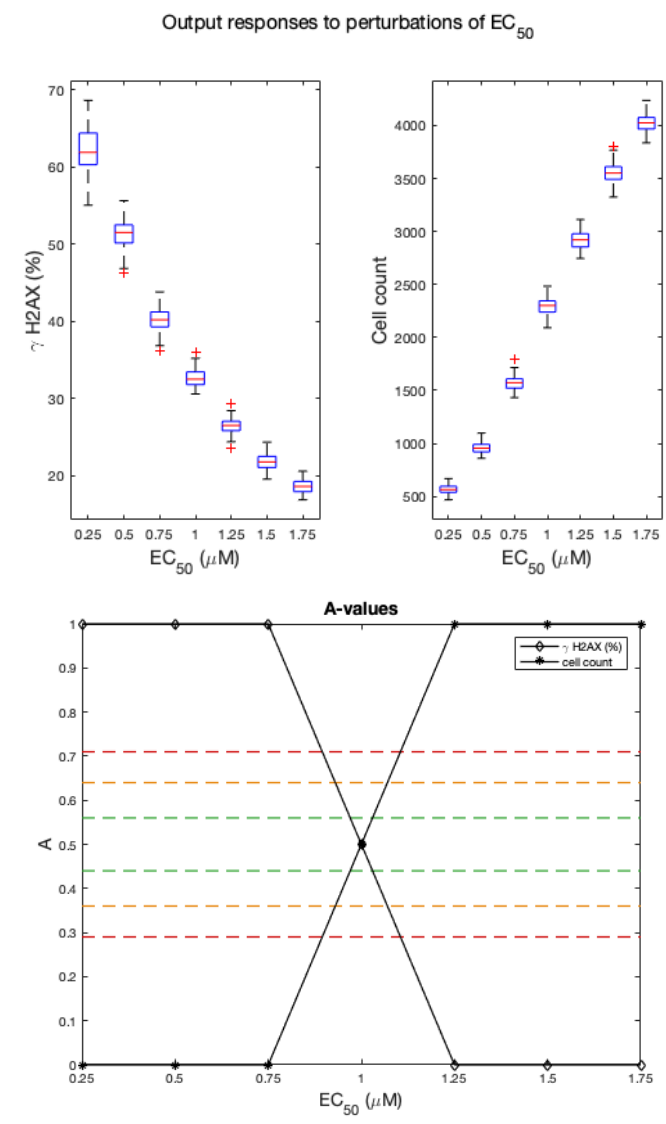

Figure 6.17: Robustness Analysis. Left: Output responses, in terms of percentage of $\gamma \mathrm{H} 2 \mathrm{AX}$ positive (i.e. damaged) cells, and cell count as a result of perturbations to the input variable $E C_{50}$. Right: Maximal $\hat{A}$-values resulting from comparisons between distributions with perturbed data and a distribution with calibrated (unperturbed) data. 

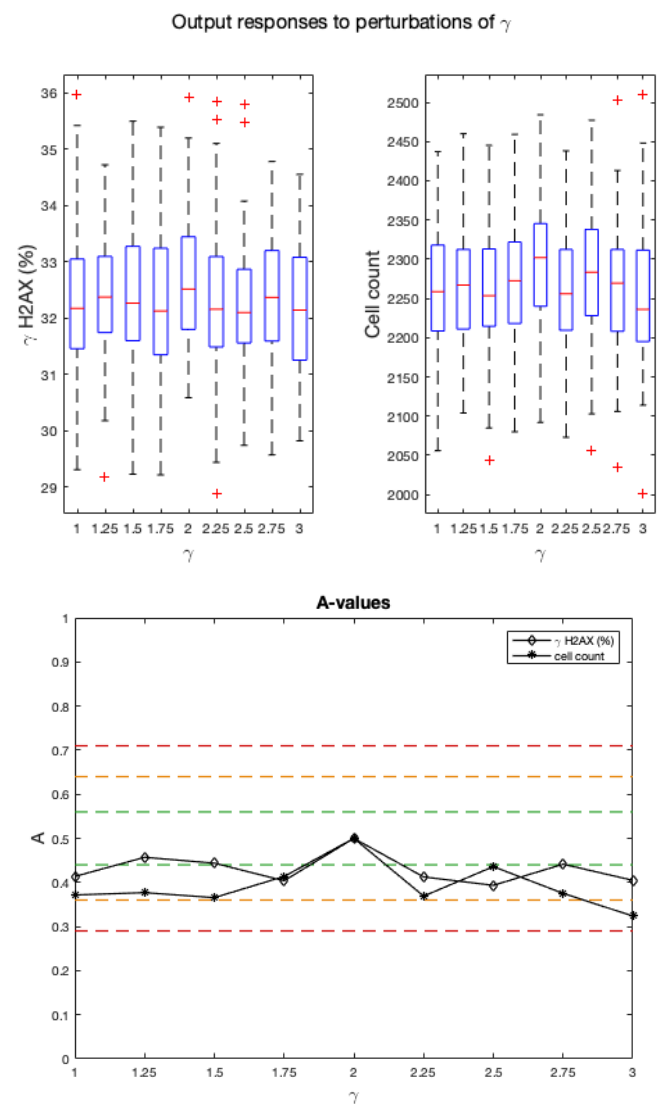

Figure 6.18: Robustness Analysis. Left: Output responses, in terms of percentage of $\gamma \mathrm{H} 2 \mathrm{AX}$ positive (i.e. damaged) cells, and cell count as a result of perturbations to the input variable $\gamma$. Right: Maximal $\hat{A}$-values resulting from comparisons between distributions with perturbed data and a distribution with calibrated (unperturbed) data. 


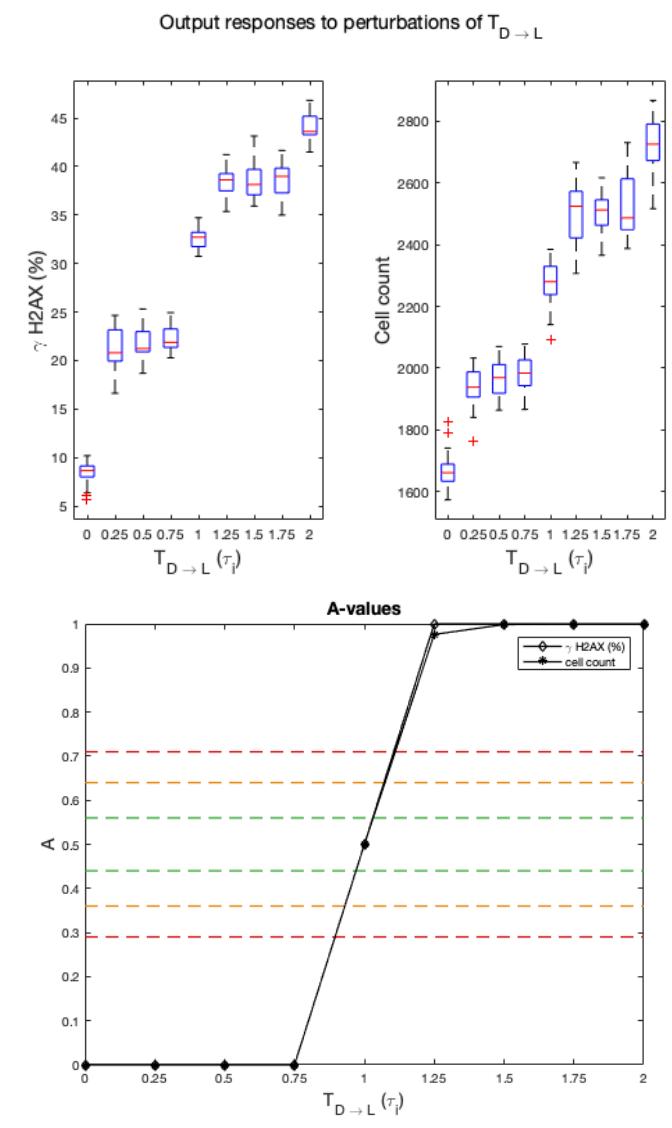

Figure 6.19: Robustness Analysis. Left: Output responses, in terms of percentage of $\gamma \mathrm{H} 2 \mathrm{AX}$ positive (i.e. damaged) cells, and cell count as a result of perturbations to the input variable $T_{D \rightarrow L}$. Right: Maximal $\hat{A}$-values resulting from comparisons between distributions with perturbed data and a distribution with calibrated (unperturbed) data. 
Correlation between output and input variable $\mu$
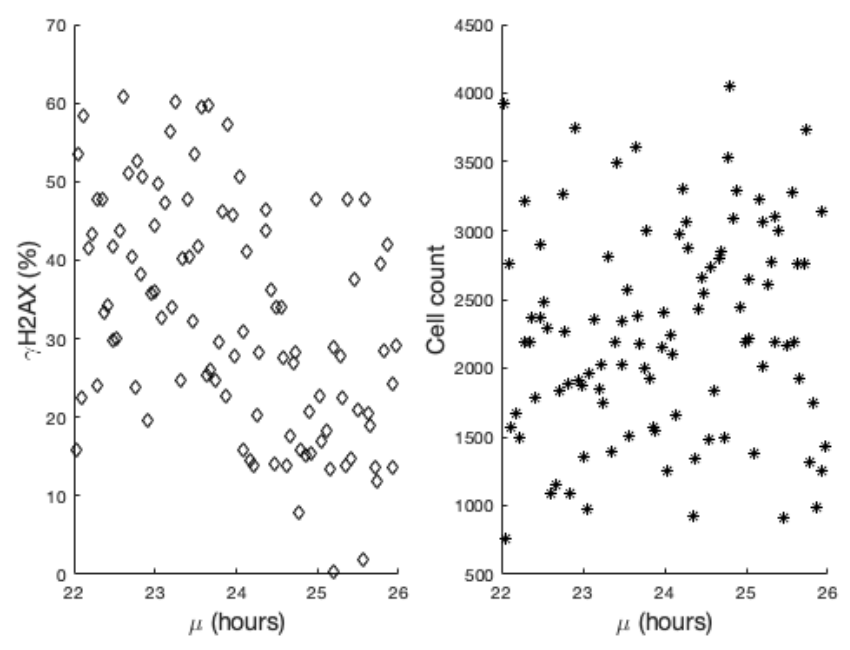

Figure 6.20: Latin Hypercube Analysis. Outputs in terms of $\gamma \mathrm{H} 2 \mathrm{AX}$ positive cells (left) and number of viable cells (right) when global parameter perturbations are performed. The scatter-plots show the correlation between outputs and the input variable $\mu$.
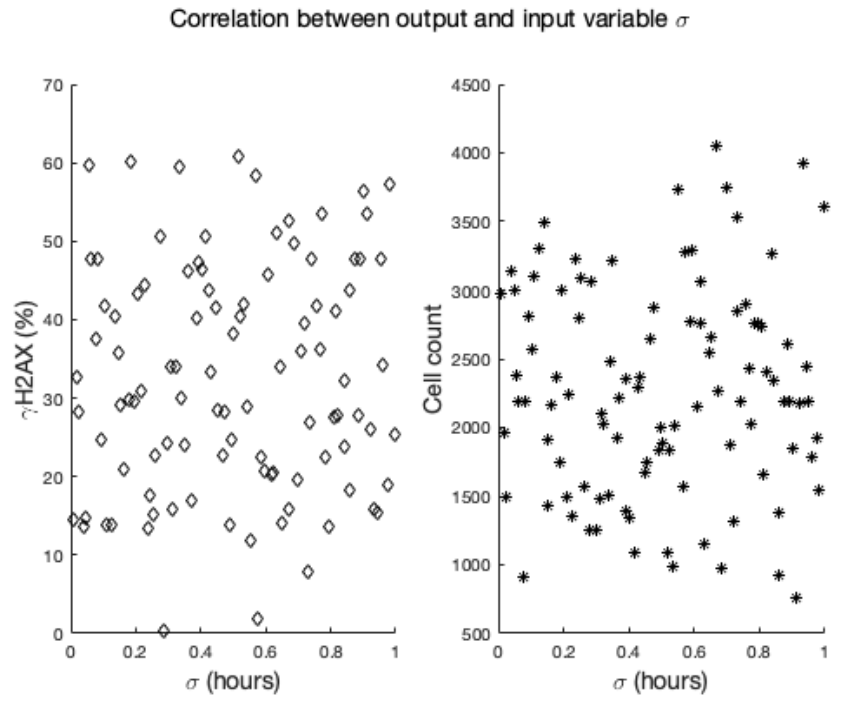

Figure 6.21: Latin Hypercube Analysis. Outputs in terms of $\gamma \mathrm{H} 2 \mathrm{AX}$ positive cells (left) and number of viable cells (right) when global parameter perturbations are performed. The scatter-plots show the correlation between outputs and the input variable $\sigma$. 


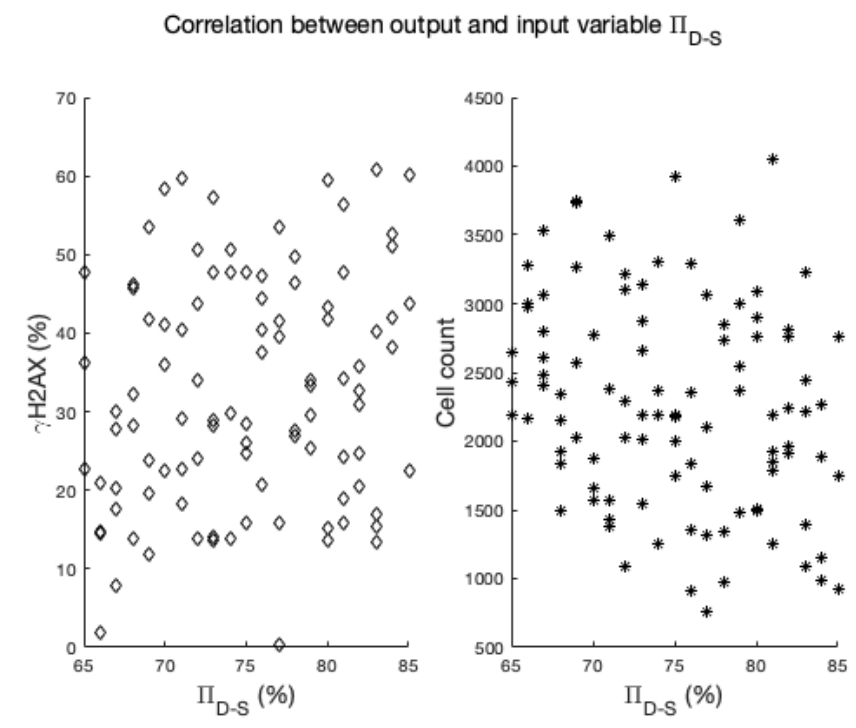

Figure 6.22: Latin Hypercube Analysis. Outputs in terms of $\gamma \mathrm{H} 2 \mathrm{AX}$ positive cells (left) and number of viable cells (right) when global parameter perturbations are performed. The scatter-plots show the correlation between outputs and the input variable $\Pi_{D-S}$.

Correlation between output and input variable $\Theta_{D-S}$
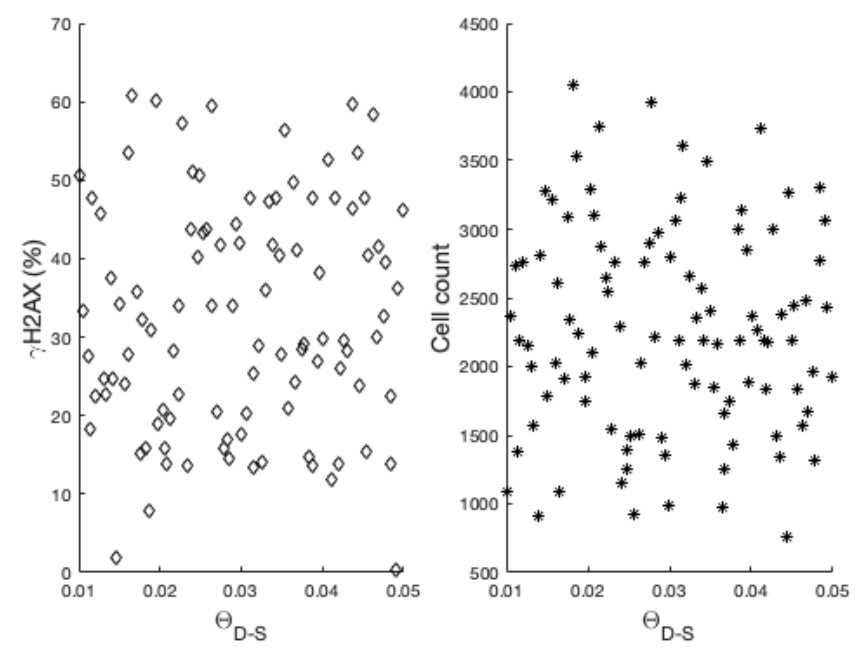

Figure 6.23: Latin Hypercube Analysis. Outputs in terms of $\gamma \mathrm{H} 2 \mathrm{AX}$ positive cells (left) and number of viable cells (right) when global parameter perturbations are performed. The scatter-plots show the correlation between outputs and the input variable $\Theta_{D-S}$. 

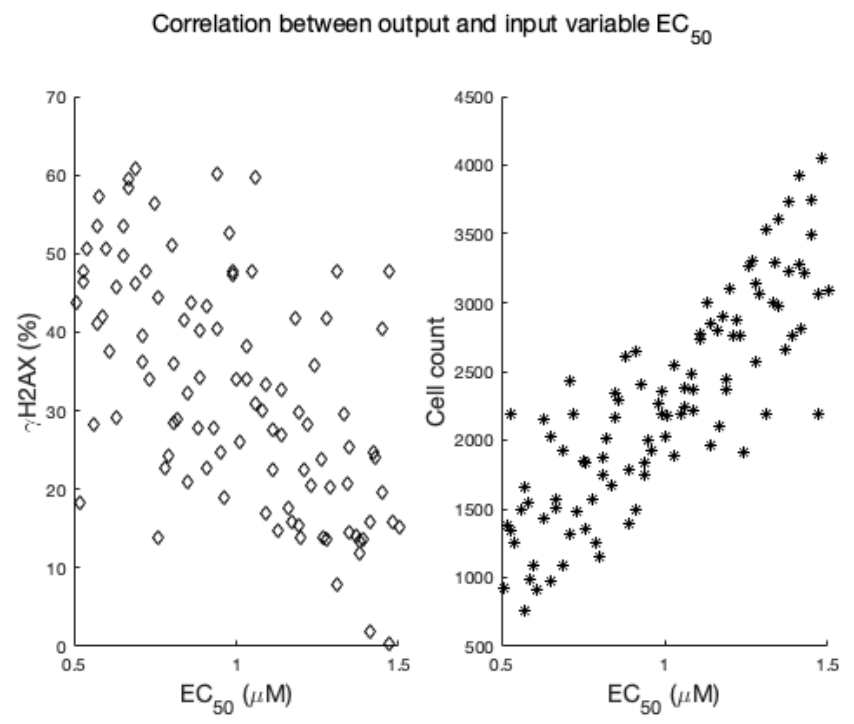

Figure 6.24: Latin Hypercube Analysis. Outputs in terms of $\gamma \mathrm{H} 2 \mathrm{AX}$ positive cells (left) and number of viable cells (right) when global parameter perturbations are performed. The scatter-plots show the correlation between outputs and the input variable $E C_{50}$.
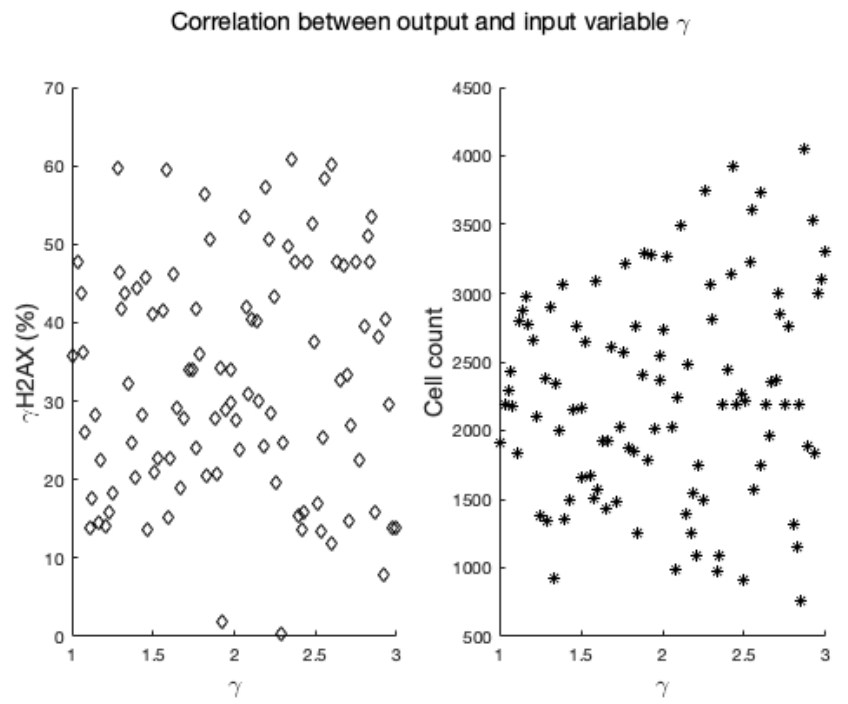

Figure 6.25: Latin Hypercube Analysis. Outputs in terms of $\gamma \mathrm{H} 2 \mathrm{AX}$ positive cells (left) and number of viable cells (right) when global parameter perturbations are performed. The scatter-plots show the correlation between outputs and the input variable $\gamma$. 
Correlation between output and input variable $\mathrm{T}_{\mathrm{L} \rightarrow \mathrm{D}}$

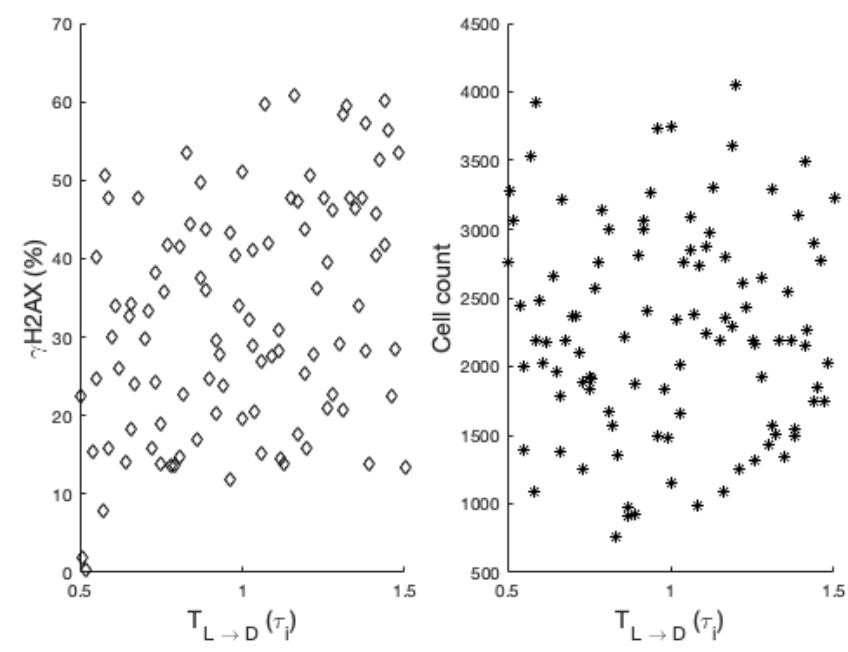

Figure 6.26: Latin Hypercube Analysis. Outputs in terms of $\gamma \mathrm{H} 2 \mathrm{AX}$ positive cells (left) and number of viable cells (right) when global parameter perturbations are performed. The scatter-plots show the correlation between outputs and the input variable $T_{L \rightarrow D}$. 


\section{Chapter 7}

\section{Conclusion}

In this thesis we discussed the role of mathematical modelling in current, and future, cancer research. We demonstrated how solid tumour models can be used to study pertinent treatment scenarios in silico. The mathematical framework used in this thesis is based on a multiscale, hybrid, cellular automaton and, as was demonstrated in the studies outlined in Chapters 3,4 and 5, this framework provides a great opportunity to study aspects of tumour dynamics and treatment responses that are not empirically observable in wet labs and clinical settings. In Chapter 5, the mathematical framework was fully calibrated by in vitro data and validated by in vivo data, signifying the potential of its use in pre-clinical settings. The mathematical framework used in this thesis can be improved by refining, expanding and/or parameterising it. More sophisticated numerical methods can be used to solve the equations describing diffusion processes and cell cycle regulation. More realistic lattice boundary conditions can be deployed in order to closer simulate in vivo tumour environments. One can also use more advanced computational techniques in situations where concerns regarding computational costs are important or in situations where one wants to simulate larger populations of agents. In research situations where sub-cellular details are of importance, more intracellular details can be included and, conversely, when such details are not important, intracellular details can be omitted. Although the mathematical framework used in this thesis can be improved in may ways, it does serve as an adaptable and easy-to-use in silico tool. As was discussed in the first chapter of this thesis, today there exist multiple mathematical models that are able to capture various aspects of cancer. Once validated, data driven, predictive mathematical models can be used as a great complement 


\section{CONCLUSION}

to traditional cancer research.

Although advancements in cancer research are being made in parallel across various research disciplines, multidisciplinary collaborations have the potential to accelerate the process of translating cancer research into applications that are successful in clinical settings. To this end, McGuire et al. [225] provide an implementable pipeline for interdisciplinary development of cancer therapies. They illustrate how to structure the workflow amongst clinicians, biologists and researchers from STEM (science, technology, engineering, mathematics) in an optimal, feasible manner. The workflow demonstrates how multidisciplinary research should alternate between being performed parallelly and sequentially. It also incorporates refining, iterative processes and an outlined order of operations which act to bring new cancer protocols to clinics as quickly and safely as possible. The concept and workflow proposed by McGuire et al. 225] acknowledges, yet transcends, practical limitations as it allows for collaboration across disciplines, distances and institutes. In order to comprehensively transfer insights from blackboardto-bedside [186], dialogue and collaboration between clinicians and mathematicians, as well as biologists and experimentalists is key [49]. This need for interdisciplinary methods is currently being pursued, integrating classical cancer research with innovative ideas from research across multiple disciplines. It is my personal aspiration to work in such interdisciplinary teams, united with a common goal to further cancer research.

Conceptually, the research field that is Mathematical Oncology can be regarded as a mathematical sub-category of oncology, or as a cancer-related sub-category of mathematics. Accordingly, the research methodology of a mathematician in the field may vary. A mathematician may choose to work with highly theoretical mathematical models that include theories that can not be (or have not been) directly validated in wetlabs. Such models may elucidate biological mechanisms and add to biological knowledge. Alternatively, a mathematician may choose to work with more practical, minimal-parameter models that are based on well established biological phenomena that are empirically observable. Such models may be more easily tested, and validated, in wet labs. Contributions to both theoretical and practical aspects of mathematical oncology are important and valid, and both research methodologies should be pursued by the research community in parallel. However, in the end, there needs to be a bridge between math- 
ematics and pre-clinical/clinical research in order for mathematical oncology to be implementable and ultimately useful in practice. After all, when it comes to mathematical oncology, the research may be mathematical but the motivation is humanitarian. 


\section{Chapter 8}

\section{Appendix}

\section{A1: Appendix to Chapter 3}

\section{Implementing Oxygen Dynamics}

The total, absolute amount of oxygen in the system fluctuates over time due to the chosen oxygen equation, parameters and boundary conditions. However scaled oxygen values are used in order to evaluate spatial oxygen distribution and determine hypoxia, these values are re-scaled at every time step [67]. In Figure 8.1 cell-maps and oxygenmaps demonstrating spatial oxygen distribution at certain times are provided, these maps are visualised using ParaView [161].

In Table 8.1, various oxygen measurements for certain time points are listed in both absolute and scaled forms. Here $\left(K_{i j}^{T}\right)$ denotes the absolute oxygen value in grid point $(i, j)$ at time $T$, and similarly $\left(\hat{K}_{i j}^{T}\right)$ denotes the scaled oxygen value in grid point $(i, j)$ at time $T$. Here $i$ and $j$ are spatial integer indices ranging from 1 to 100 as a square grid with $100^{2}$ grid points is used. For absolute oxygen values these listed measurements are specifically; the total amount of oxygen in the system $\left(\sum_{i j} K_{i j}^{T}\right)$, the average oxygen value at one grid point $\left(\bar{K}_{i j}^{T}\right)$, the oxygen value at the grid point with the maximum amount of oxygen $\left(\max \left(K_{i j}^{T}\right)\right)$ and the oxygen value at the grid point with the minimum amount of oxygen $\left(\min \left(K_{i j}^{T}\right)\right)$. The same measurements are done for scaled oxygen values, using the hat notation to denote that the values are scaled. 


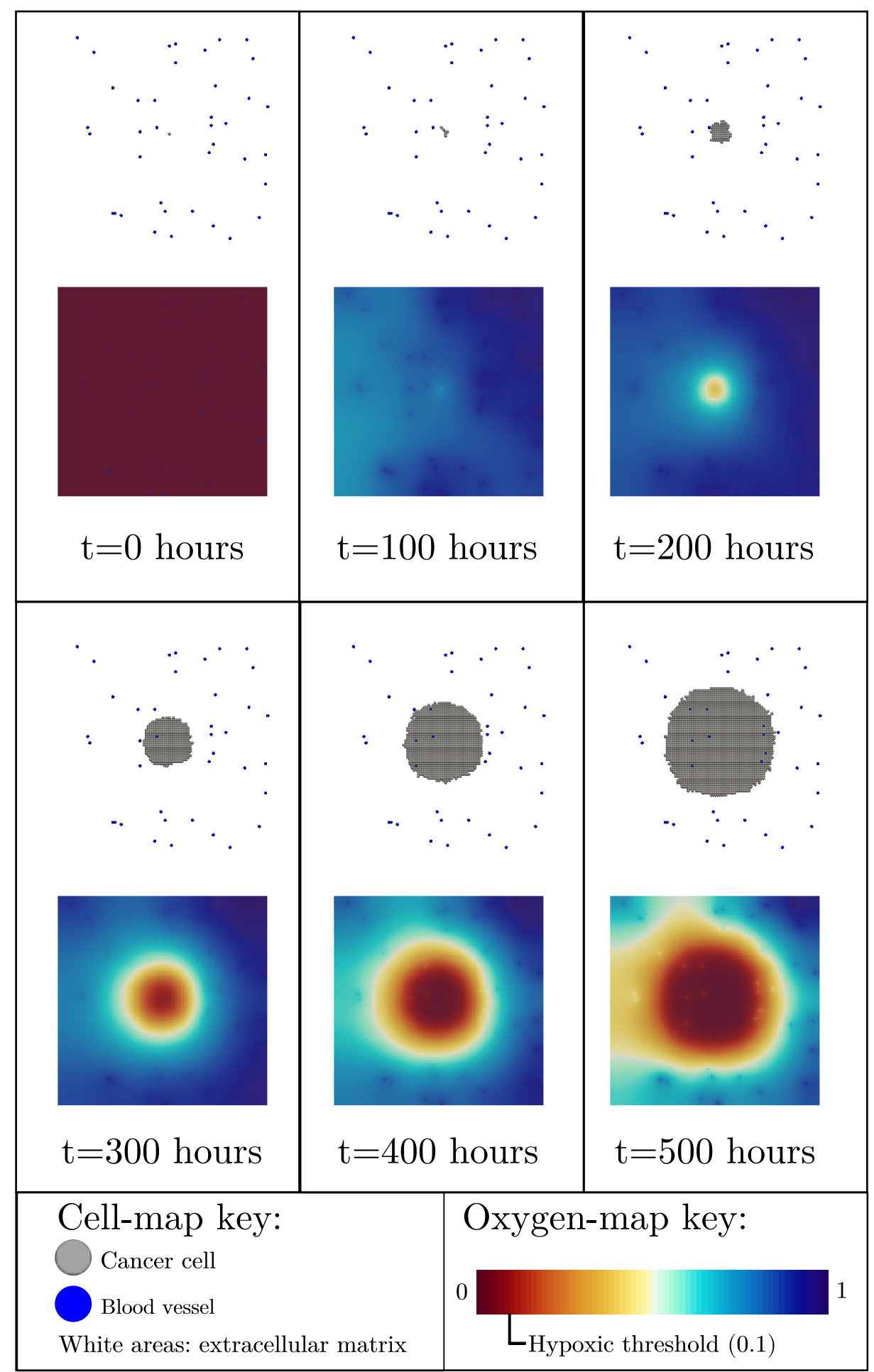

Figure 8.1: Cell-maps (top) and oxygen-maps (bottom) at certain times pre chemotherapy administration. The oxygen-maps demonstrate the spatial oxygen distribution in terms of scaled, nondimensionalised oxygen values ranging between 0 and 1 at each grid point. 


\begin{tabular}{|c|c|c|c|c|c|c|c|c|}
\hline time $[\mathrm{h}]$ & $\begin{array}{l}\sqrt{2} \\
i=2 \\
15: 5\end{array}$ & E: & 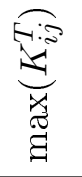 & 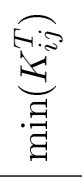 & 先 & 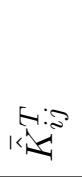 & 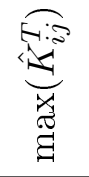 & 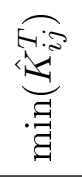 \\
\hline 0 & 0.41 & 0 & 0.01 & 0.00 & 40.05 & 0.00 & 1.00 & 0.00 \\
\hline 100 & 41048.10 & 4.10 & 4.92 & 3.50 & 8334.76 & 0.83 & 1.00 & 0.71 \\
\hline 200 & 70535.20 & 7.05 & 8.30 & 3.36 & 8498.16 & 0.85 & 1.00 & 0.40 \\
\hline 300 & 58273.21 & 5.83 & 7.75 & 0.69 & 7514.37 & 0.75 & 1.00 & 0.09 \\
\hline 400 & 29163.37 & 2.92 & 4.74 & 0.09 & 6155.72 & 0.62 & 1.00 & 0.02 \\
\hline 500 & 12093.96 & 1.21 & 2.62 & 0.02 & 4619.06 & 0.46 & 1.00 & 0.01 \\
\hline
\end{tabular}

Table 8.1: Nondimensionalised oxygen values of the system at certain time points, in both absolute form and scaled form (hat notation).

No DR: Tests (a1) to (a4)

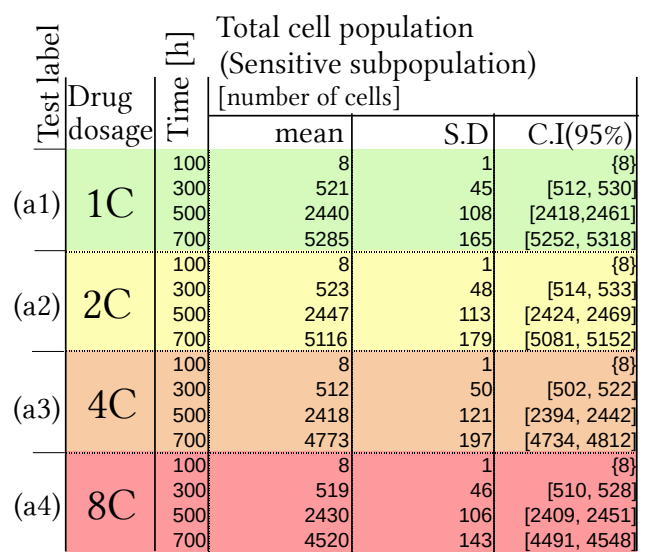

\section{Result Data}

This section provides listings of the mean value (mean), standard deviation (S.D) and $95 \%$-confidence interval (I.C(95\%)) for test results (a1) through to (e4), presented by graphs in Figure 4. Here all values have been rounded to integers, corresponding to full number of cells.

\section{Robustness Analysis}

To verify that our results are robust in regards to the chosen parameters listed in Table 3.3, a sensitivity investigation of critical parameters is preformed in which parameters are varied, one at a time, according to Table 8.2. Each such sensitivity test is performed 100 times, results are provided in Figure S7. These results show that our qualitative 
Primary DR: Tests (b1) to (b4)

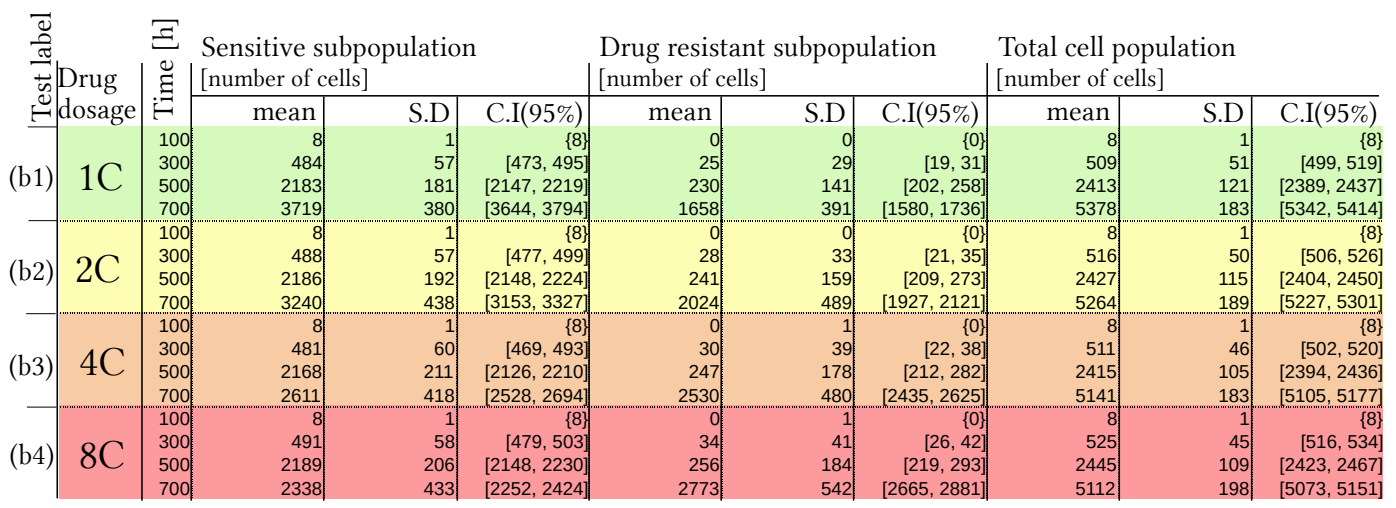

Induced DR: Tests (c1) to (c4)

\begin{tabular}{|c|c|c|c|c|c|c|c|c|c|c|c|}
\hline 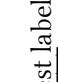 & Drug & 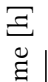 & $\begin{array}{l}\text { Sensitive s } \\
\text { [number of } \mathrm{c}\end{array}$ & slatio & & $\begin{array}{l}\text { Drug resis } \\
\text { [number of }\end{array}$ & bpop & lation & $\begin{array}{l}\text { Total cell } \mathrm{p} \\
\text { [number of } \mathrm{c}\end{array}$ & tion & \\
\hline & dosage & 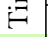 & mean & S.D & C.I $(95 \%)$ & mean & S.D & C.I $(95 \%)$ & mean & S.D & C.I $(95 \%)$ \\
\hline & & $\begin{array}{l}100 \\
300\end{array}$ & $\begin{array}{r}8 \\
520\end{array}$ & $\begin{array}{r}1 \\
46\end{array}$ & $\begin{array}{r}\{8\} \\
{[510,529]}\end{array}$ & $\begin{array}{l}0 \\
0\end{array}$ & 0 & $\begin{array}{l}\{0\} \\
\{0\}\end{array}$ & $\begin{array}{r}8 \\
520\end{array}$ & $\begin{array}{r}1 \\
46\end{array}$ & $\begin{array}{r}\{8\} \\
{[510,529]}\end{array}$ \\
\hline (c1) & 10 & 500 & 2435 & 106 & {$[2414,2456]$} & 0 & 0 & $\{0\}$ & 2435 & 106 & {$[2414,2456]$} \\
\hline & & 700 & 2828 & 155 & {$[2798,2859]$} & 2560 & 134 & {$[2533,2586]$} & 5388 & 171 & {$[5354,5422]$} \\
\hline & & 100 & 8 & 1 & $\{8\}$ & 0 & 0 & $\{0\}$ & 8 & 1 & $\{8\}$ \\
\hline & & 300 & 519 & 49 & {$[509,529]$} & 0 & 0 & $\{0\}$ & 519 & 49 & {$[509,529]$} \\
\hline (c2) & & 500 & 2436 & 115 & {$[2414,2459]$} & 0 & 0 & $\{0\}$ & 2436 & 115 & {$[2414,2459]$} \\
\hline & & 700 & 3006 & 156 & {$[2975,3037]$} & 2167 & 149 & {$[2137,2197]$} & 5173 & 182 & {$[5137,5209]$} \\
\hline & & 100 & 8 & 1 & & 0 & 0 & $\{0\}$ & 8 & 1 & $\{8\}$ \\
\hline & & 300 & 518 & 48 & {$[509,527]$} & 0 & 0 & $\{0\}$ & 518 & 48 & {$[509,527]$} \\
\hline (c3 & $4 \mathrm{C}$ & 500 & 2430 & 107 & {$[2409,2451]$} & 0 & 0 & $\{0\}$ & 2430 & 107 & {$[2409,2451]$} \\
\hline & & 700 & 3662 & 147 & {$[3632,3691]$} & 1200 & 172 & {$[1166,1234]$} & 4861 & 172. & {$[4827,4895]$} \\
\hline & & 100 & 8 & 1 & & 0 & 0 & $\{0\}$ & 8 & 1 & \\
\hline$(c 4)$ & 8 & 300 & 519 & 50 & {$[509,529]$} & & 0 & $\{0\}$ & 519 & 50 & {$[509,529]$} \\
\hline & ชC & 500 & 2432 & 120 & {$[2408,2456]$} & 0 & 0 & $\{0\}$ & 2432 & 120 & {$[2408,2456]$} \\
\hline & & 700 & 3929 & 201 & {$[3889,3968]$} & 638 & 127 & {$[613,663]$} & 4567 & 183 & {$[4531,4603]$} \\
\hline
\end{tabular}

ICC DR: Tests (d1) to (d4)

\begin{tabular}{|c|c|c|c|c|c|c|c|c|c|c|c|}
\hline \multirow{2}{*}{ 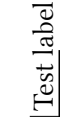 } & \multirow{2}{*}{$\left|\begin{array}{l}\text { Drug } \\
\text { dosage }\end{array}\right|$} & \multirow{2}{*}{ 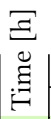 } & \multicolumn{3}{|c|}{$\begin{array}{l}\text { Sensitive subpopulation } \\
\text { [number of cells] }\end{array}$} & \multicolumn{3}{|c|}{$\begin{array}{l}\text { Drug resistant subpopulation } \\
\text { [number of cells] }\end{array}$} & \multicolumn{3}{|c|}{$\begin{array}{l}\text { Total cell population } \\
\text { [number of cells] }\end{array}$} \\
\hline & & & mean & S.D & C.I $(95 \%)$ & mean & S.D & C.I $(95 \%)$ & mean & S.D & C.I $(95 \%)$ \\
\hline \multirow{4}{*}{ (d1) } & & 100 & & 1 & & 0 & 0 & $\{0\}$ & & 1 & $\{8\}$ \\
\hline & & 300 & 527 & 45 & {$[518,536]$} & 0 & 0 & $\{0\}$ & 527 & 45 & {$[518,536]$} \\
\hline & & 500 & 2446 & 108 & {$[2425,2467]$} & 0 & 0 & $\{0\}$ & 2446 & 108 & {$[2425,2467]$} \\
\hline & & 700 & 2507 & 471 & {$[2414,2600]$} & 2910 & 484 & {$[2814,3006]$} & 5417 & 156 & {$[5386,5448]$} \\
\hline \multirow{4}{*}{ (d2) } & & 100 & 8 & 1 & $\{8\}$ & 0 & 0 & $\{0\}$ & 8 & 1 & $\{8\}$ \\
\hline & 20 & 300 & 518 & 55 & {$[507,529]$} & 0 & 0 & $\{0\}$ & 518 & 55 & {$[507,529]$} \\
\hline & & 500 & 2430 & 127 & {$[2405,2455]$} & 0 & 0 & $\{0\}$ & 2430 & 127 & {$[2405,2455]$} \\
\hline & & 700 & 2695 & 403 & {$[2615,2775]$} & 2480 & 398 & {$[2401,2559]$} & 5174 & 194 & {$[5136,5212]$} \\
\hline \multirow{4}{*}{ (d3) } & & 100 & 8 & 1 & $\{8\}$ & 0 & 0 & $\{0\}$ & 8 & 1 & $\{8\}$ \\
\hline & & 300 & 518 & 48 & {$[508,528]$} & 0 & 0 & $\{0\}$ & 518 & 48 & {$[508,528]$} \\
\hline & & 500 & 2429 & 108 & {$[2408,2450]$} & 0 & 0 & $\{0\}$ & 2429 & 108 & {$[2408,2450]$} \\
\hline & & 700 & 3428 & 340 & {$[3361,3495]$} & 1427 & 378 & {$[1352,1502]$} & 4855 & 164 & {$[4822,4888]$} \\
\hline \multirow{4}{*}{ (d4) } & & 100 & 8 & 1 & & 0 & 0 & $\{0\}$ & 8 & 1 & $\{8\}$ \\
\hline & & 300 & 517 & 47 & {$[508,526]$} & 0 & 0 & $\{0\}$ & 517 & 47 & {$[508,526]$} \\
\hline & $8 \mathrm{C}$ & 500 & 2429 & 114 & {$[2406,2452]$} & 0 & 0 & $\{0\}$ & 2429 & 114 & {$[2406,2452]$} \\
\hline & & 700 & 3740 & 320 & {$[3677,3803]$} & 830 & 309 & {$[769,891]$} & 4750 & 148 & {$[4721,4779]$} \\
\hline
\end{tabular}




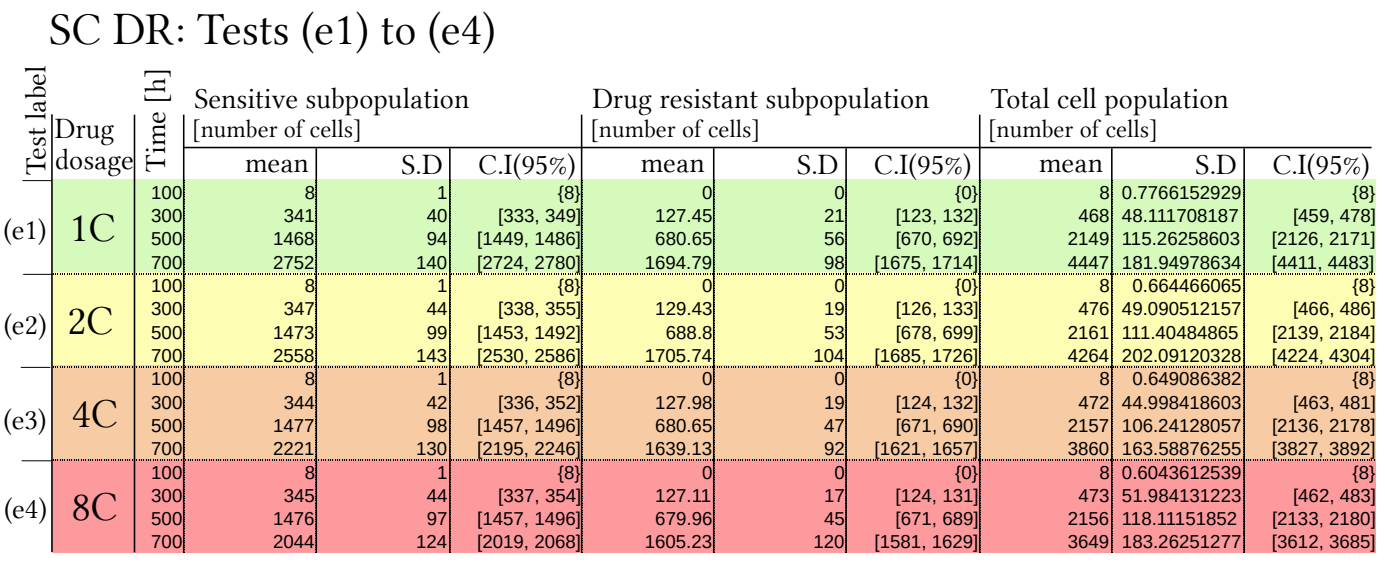

findings, concerning drug response in cancer cell populations hosting various types of drug resistance, hold for parameter variations. Indeed the ratio of drug resistant cells increases with high drug dosages in cases where drug resistance precedes chemotherapy, here in experiments (b) Primary DR and (e) SC DR. Conversely drug-induced drug resistant subpopulations are promoted in scenarios with low drug dosages, here in experiments (c) Induced DR and (d) ICC DR.

\begin{tabular}{|c|c|c|c|c|c|c|}
\hline Test & Parameter & 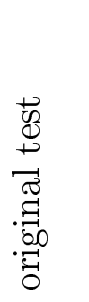 & 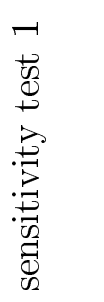 & 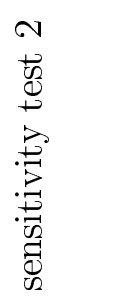 & 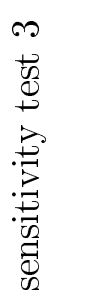 & 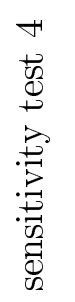 \\
\hline Primary DR & $\alpha_{p r i}$ & 0.01 & 0.005 & 0.025 & & \\
\hline Induced DR & $\chi_{i n d}$ & $\kappa / 5$ & $\kappa / 5$ & $\kappa / 5$ & $\kappa / 10$ & $\kappa / 2$ \\
\hline Induced DR & $\tau$ [minutes] & 30 & 15 & 60 & 30 & 30 \\
\hline ICC DR & $\alpha_{e x}$ & 0.05 & 0.03 & 0.1 & & \\
\hline ICC DR & $\chi_{i n d}$ & $\kappa / 5$ & $\kappa / 5$ & $\kappa / 5$ & & \\
\hline ICC DR & $\tau$ [minutes] & 30 & 30 & 30 & & \\
\hline SC DR & $\alpha_{S C}$ & 0.07 & 0.03 & 0.1 & & \\
\hline
\end{tabular}

Table 8.2: Parameters used in testing the robustness of the model. 

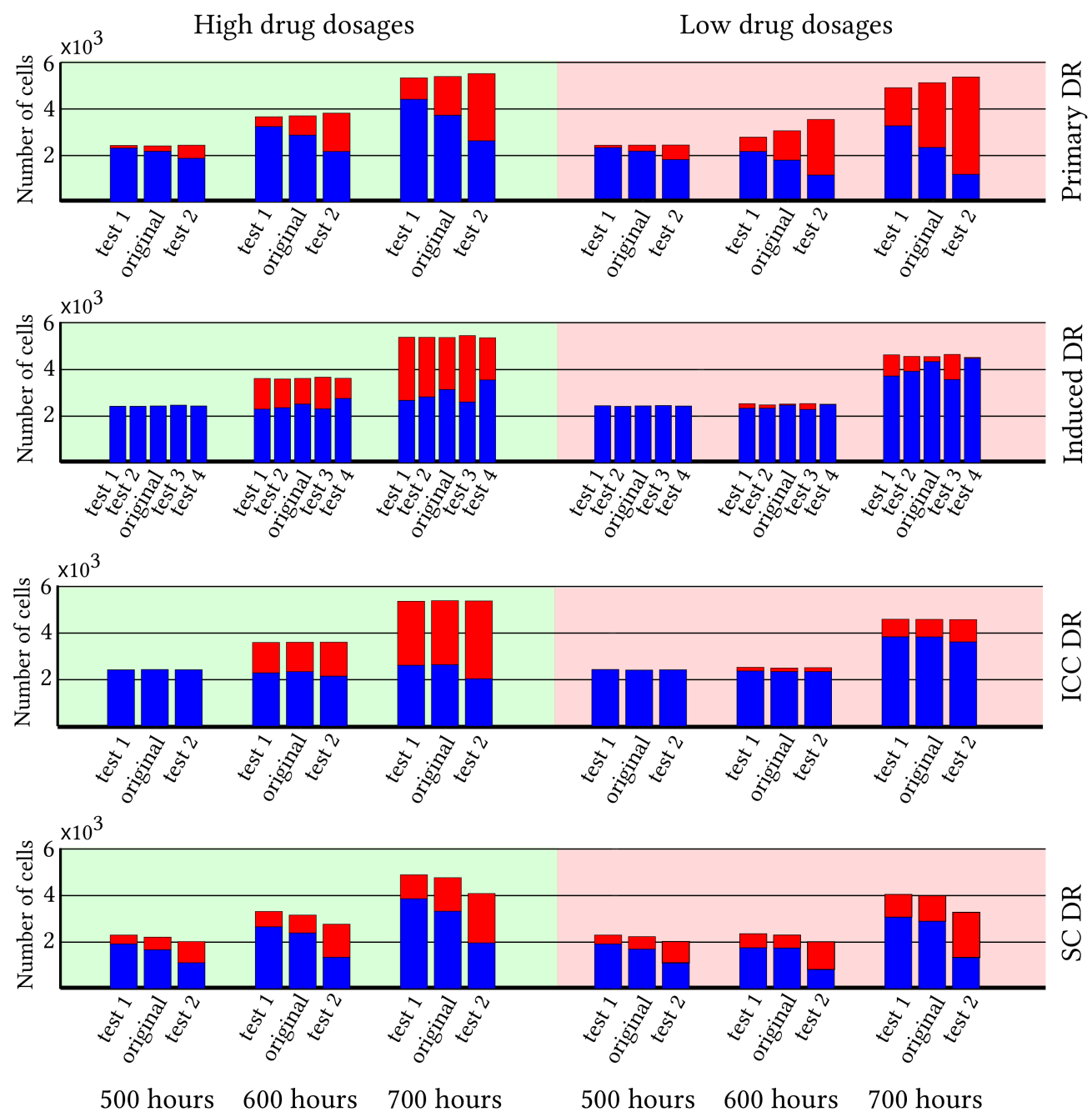

Figure 8.2: Sensitivity analysis, showing the number of sensitive (blue) and drug resistant (red) cells at three time points when low (left) and high (right) drug dosages are administered, namely $1 \mathrm{C}$ and $8 \mathrm{C}$ respectively. Each test is performed 100 times and the parameters used in each test are listed in Table 8.2. Thus for Primary DR, only the parameter $\alpha_{p r i}$ is varied. For Induced DR, $\chi_{i n d}$ and $\tau$ are both varied, one at a time, according to Table 8.2 For ICC DR only $a_{e x}$ is varied and similarly for SC DR only $a_{S C}$ is varied. 


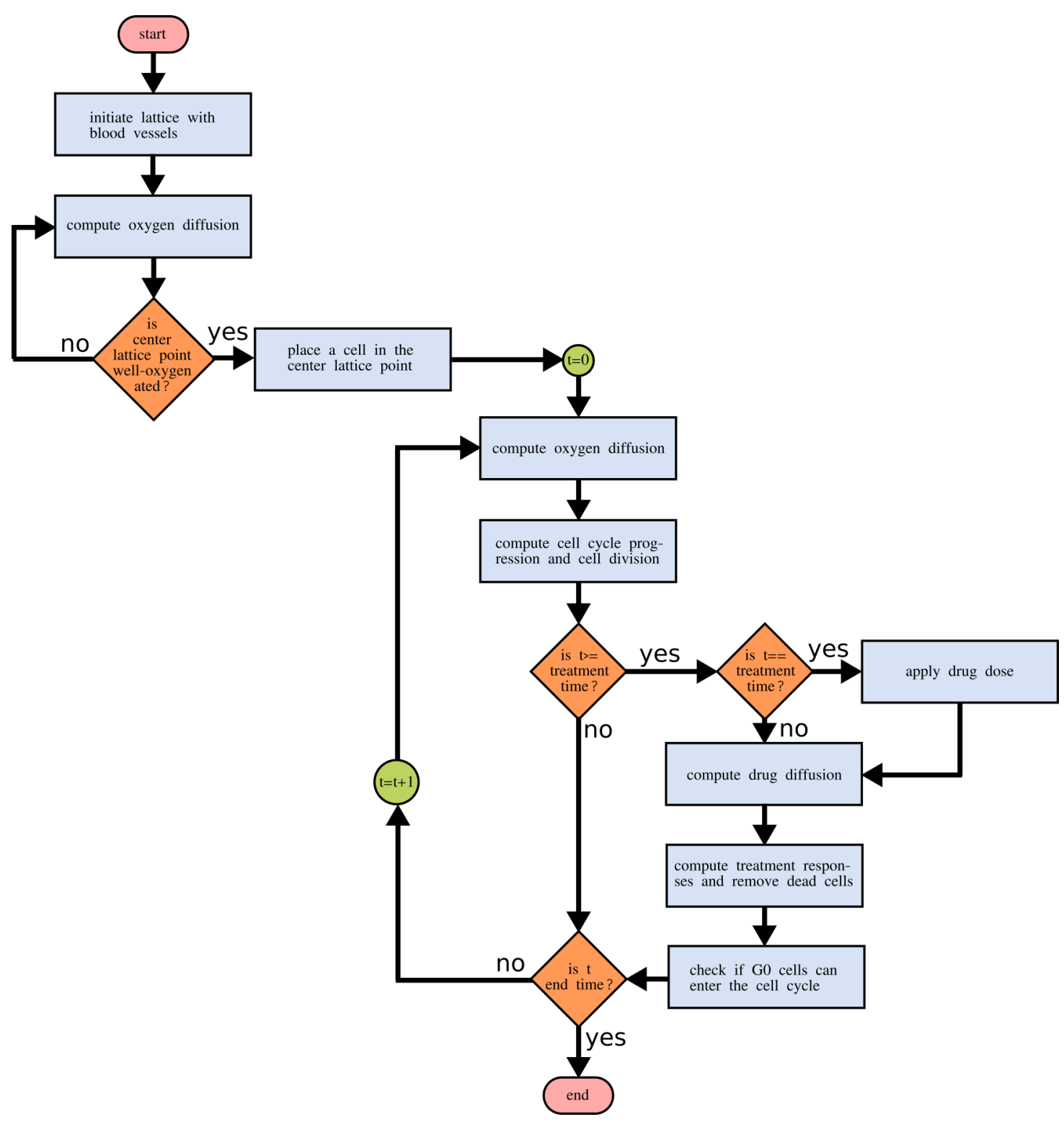

Figure 8.3: A flowchart of the code used in Chapter 3.

A flowchart of the code used in Chapter 3.

\section{A2: Appendix to Chapter 4}

Complement to Figure 4.9

Figures 8.4 and 8.5 show that the Scheduling-Experiment, performed in Section 4.4 .2 and with results provided in Figure 4.9, are qualitatively the same if a damaged cell is 
instantly removed from the lattice (Figure 8.4) or if a damaged cell is moved from the lattice after a time period corresponding to its doubling time (Figure 8.5).
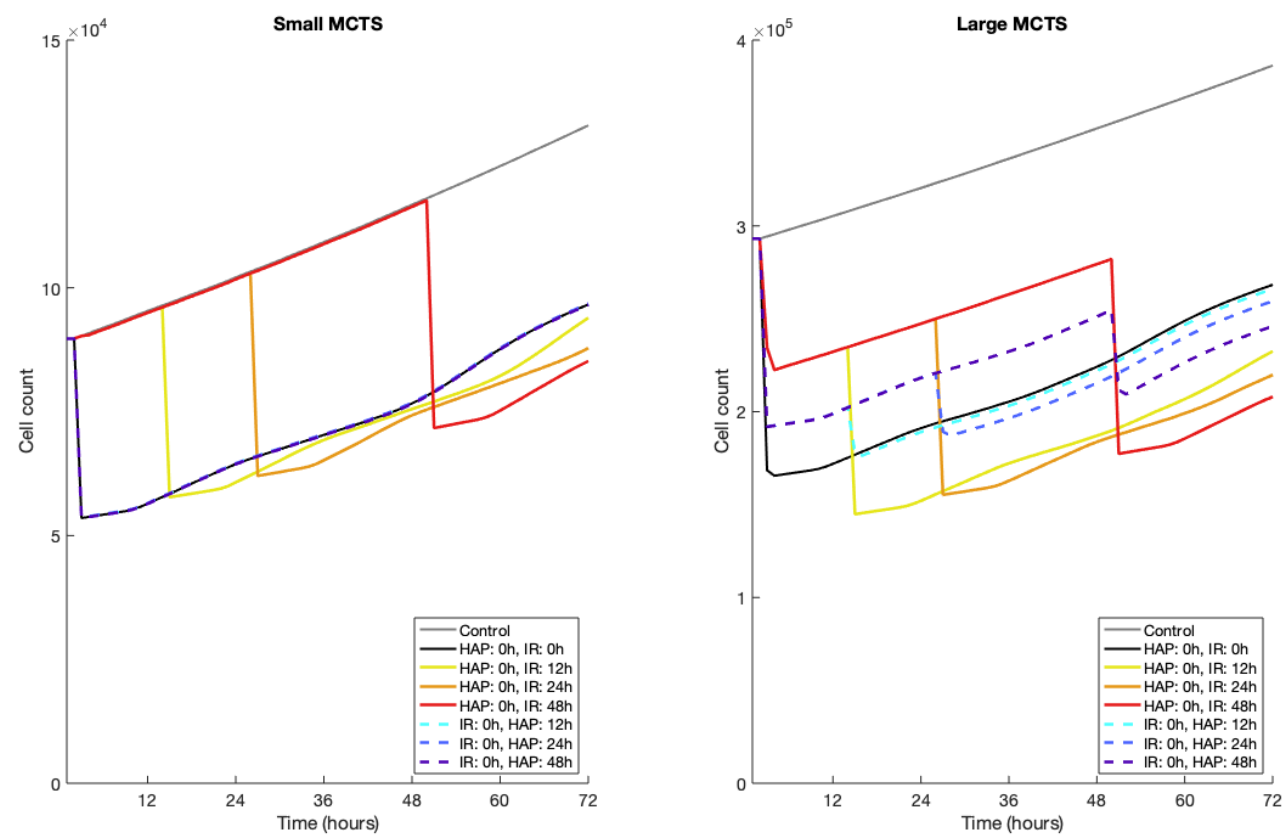

Figure 8.4: Scheduling of HAP-IR combination treatments, Complement to Figure 4.9. Cells are removed from the lattice instantaneously after the lethal event occurred.

\section{Complement to Figure 4.10}

Figures 8.6 and 8.7 show that the experiment that investigates if HAPs act as radiotherapy enhancers, discussed in Section 4.4 .3 and with results provided in Figure 4.10, are qualitatively the same if a damaged cell is instantly removed from the lattice (Figure 8.6 or if a damaged cell is moved from the lattice after a time period corresponding to its doubling time (Figure 8.7.

\section{A flowchart of the code used in Chapter 4.}

\section{A3: Appendix to Chapter 5}

Flowcharts of the codes used in Chapter 5. 

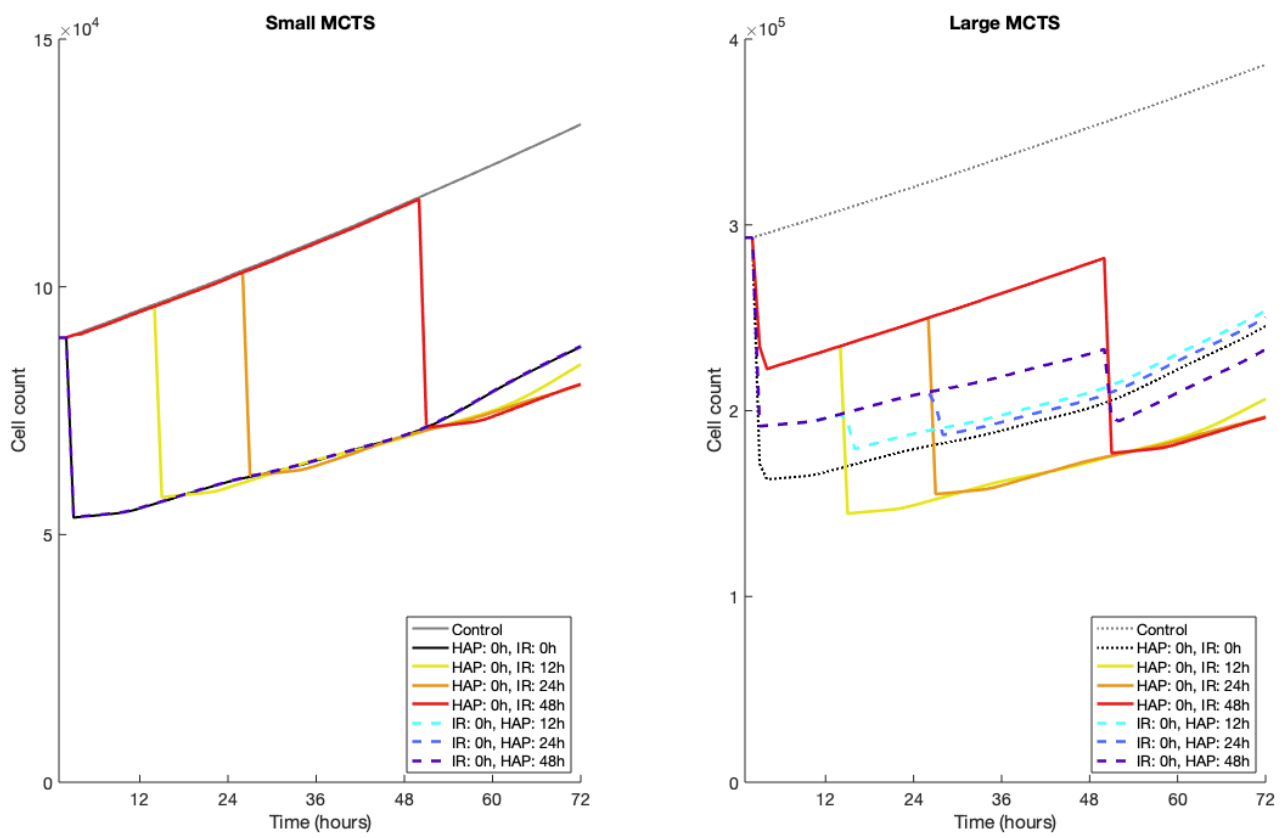

Figure 8.5: Scheduling of HAP-IR combination treatments, Complement to Figure 4.9 Cells are removed from the lattice after a time corresponding to their doubling time $\left(\tau_{i}\right)$ post the lethal event occurred. 

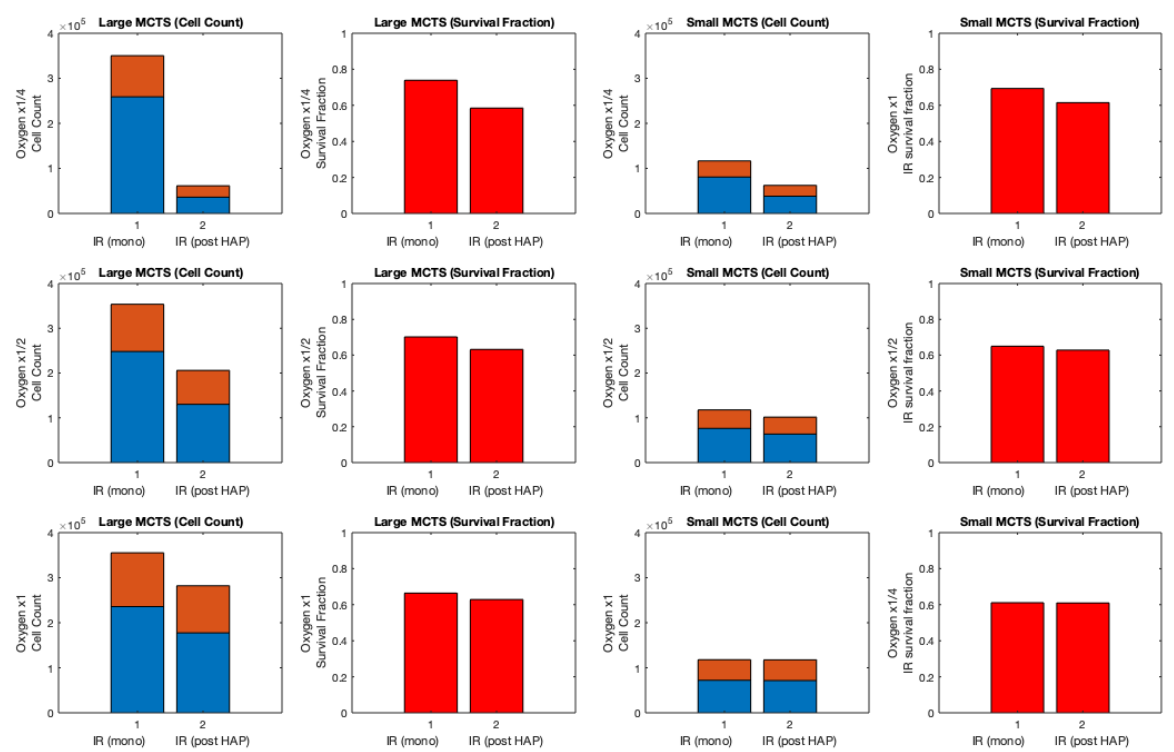

Figure 8.6: Treatment responses of radiotherapy in various MCTSs when either (1) an IR monotherapy dose is administered at $T_{0}+48$ hours or (2) IR is given at $T_{0}+48$ hours following a prior HAP dose at time $T_{0}$. Complement to Figure 4.9. Cells are removed from the lattice instantaneously after the lethal event occurred. 


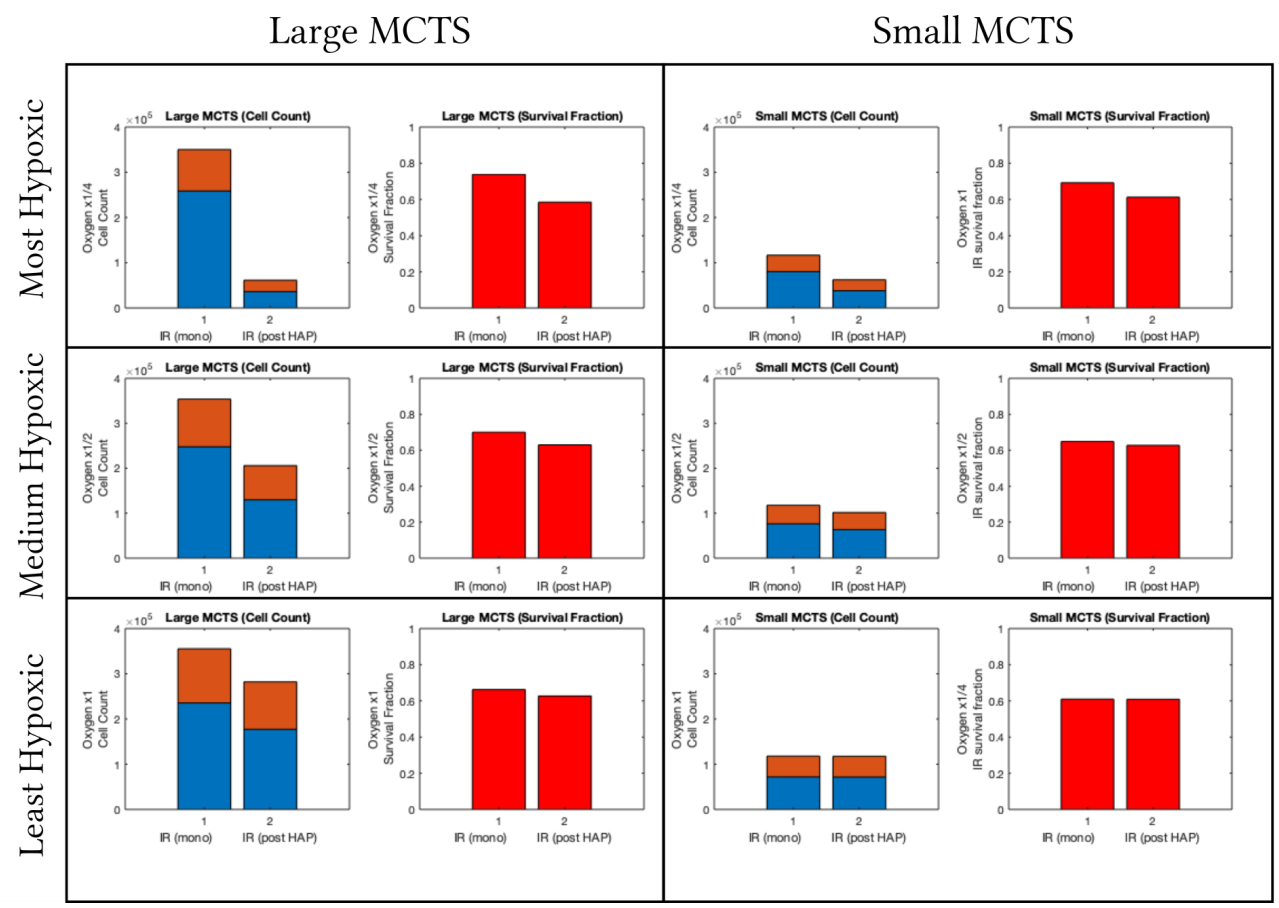

Figure 8.7: Treatment responses of radiotherapy in various MCTSs when either (1) an IR monotherapy dose is administered at $T_{0}+48$ hours or (2) IR is given at $T_{0}+48$ hours following a prior HAP dose at time $T_{0}$. Complement to Figure 4.10. Cells are removed from the lattice after a time corresponding to their doubling time $\left(\tau_{i}\right)$ post the lethal event occurred. 


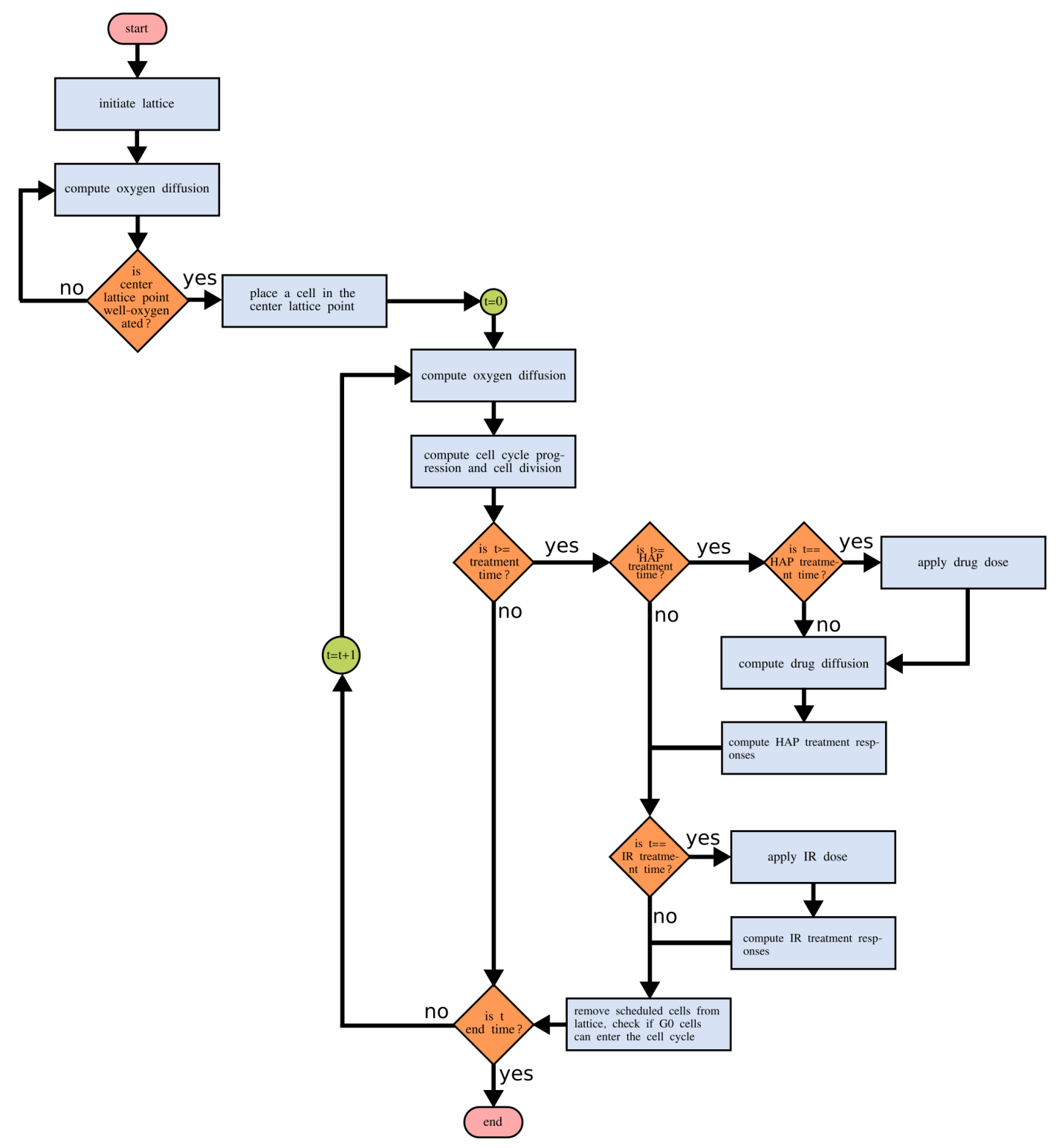

Figure 8.8: A flowchart of the code used in Chapter 4. 


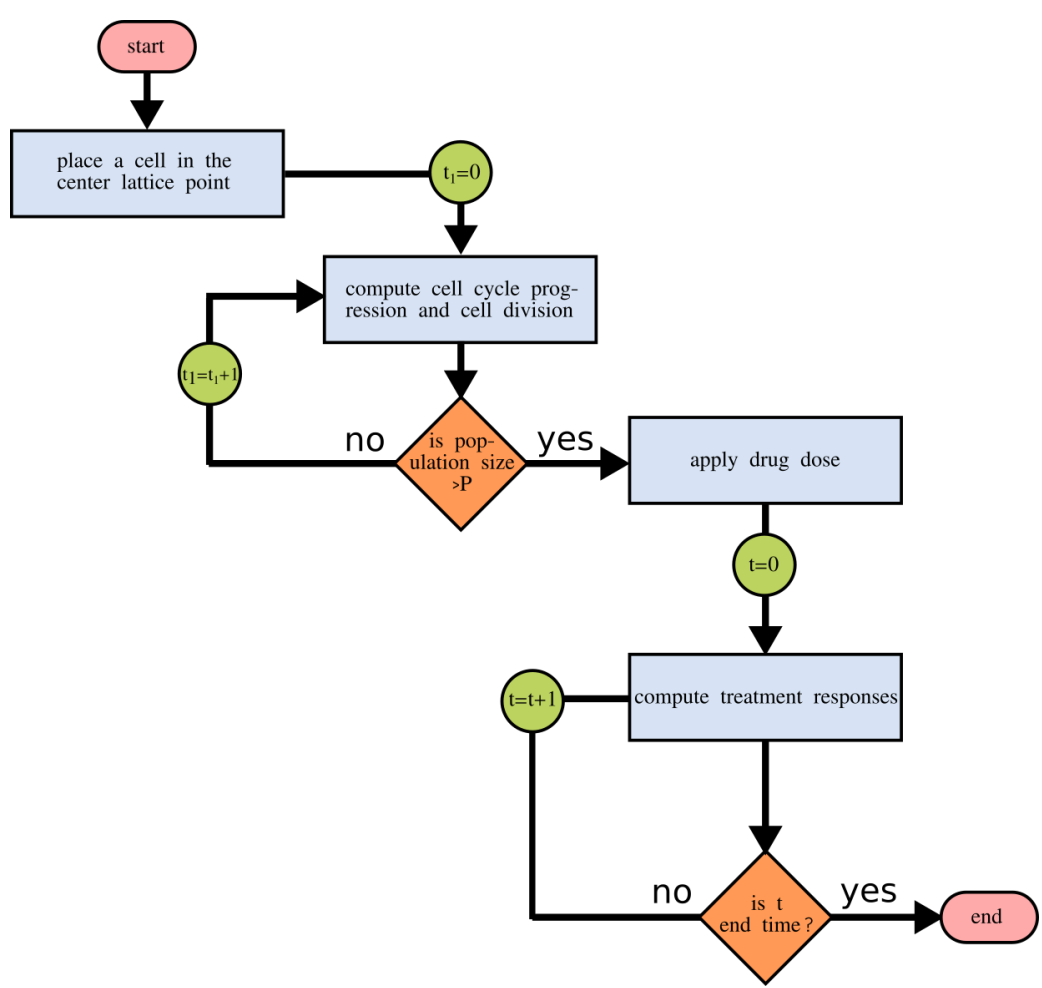

Figure 8.9: A flowchart of the code used in Chapter 5 simulating in vitro scenarios. 


\section{APPENDIX}

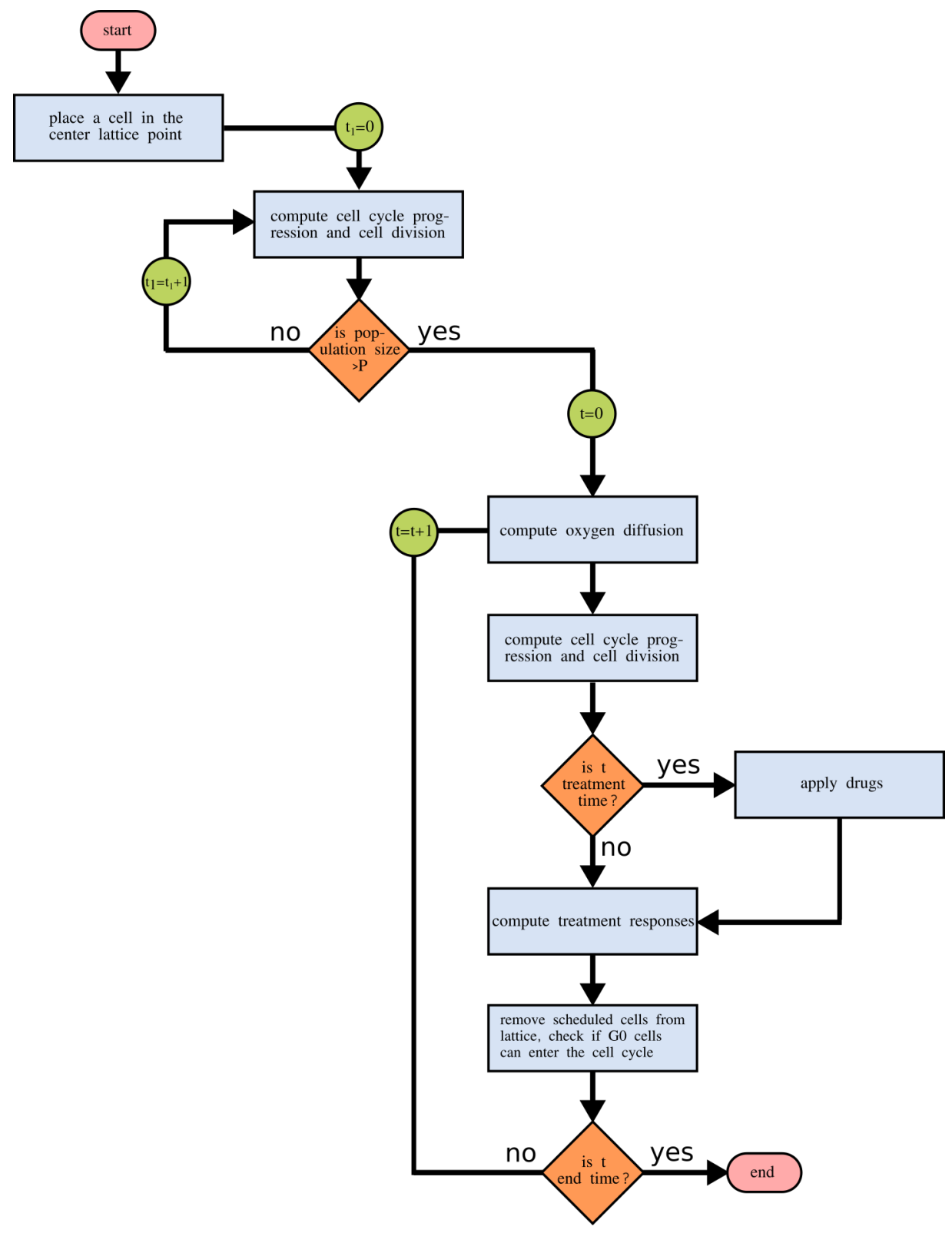

Figure 8.10: A flowchart of the code used in Chapter 5 simulating in vivo scenarios. 


\section{References}

[1] T. Alarcon, H. M. Byrne, and P. K. Maini, "A mathematical model of the effects of hypoxia on the cell-cycle of normal and cancer cells," J. Theor. Biol. 229 no. 3, (Aug, 2004) 395-411. xvi, 17, 18, 78, 109, 110, 111

[2] H. Kempf, H. Hatzikirou, M. Bleicher, and M. Meyer-Hermann, "In silico analysis of cell cycle synchronisation effects in radiotherapy of tumour spheroids," PLoS Comput. Biol. 9 no. 11, (2013) e1003295. xvi, 34, 35, 36

[3] A. Voissiere, E. Jouberton, E. Maubert, F. Degoul, C. Peyrode, J. M. Chezal, and E. Miot-Noirault, "Development and characterization of a human three-dimensional chondrosarcoma culture for in vitro drug testing," PLoS ONE 12 no. 7, (2017) e0181340. $\mathrm{xix}, \mathrm{xx}, 19,71,75,76,77,78,79,80,81,87,95,99$

[4] K. Arnold, J. Gosling, and D. Holmes, The Java programming language. Addison Wesley Professional, 2005. xix, 77

[5] S. Checkley, L. MacCallum, J. Yates, P. Jasper, H. Luo, J. Tolsma, and C. Bendtsen, "Bridging the gap between in vitro and in vivo: Dose and schedule predictions for the ATR inhibitor AZD6738," Sci Rep 5 (Aug, 2015) 13545. xxi. xxii, xxviii, 2, 18, 19, 103, 104, 105, 106, 107, 108, 109, 111, 112, 115, 120, 121, $122,123,124,125$

[6] G. G. Powathil, K. E. Gordon, L. A. Hill, and M. A. Chaplain, "Modelling the effects of cell-cycle heterogeneity on the response of a solid tumour to chemotherapy: biological insights from a hybrid multiscale cellular automaton model," J. Theor. Biol. 308 (Sep, 2012) 1-19. xxvii, 7, 10, 11, 12, 15, 17, 19, 21, $42,43,45,46,47,48,49,53,57,63,76,78,60,84,109,110,111,112,125$ 


\section{REFERENCES}

[7] M. M. Dale and H. P. Rang, "Rang \& Dale's pharmacology ," Edinburgh: Churchill Livingstone (2007) · xxvii, 10, 20, 22, 23, 24, 25, 26, 27, 47, 83, 112. 113

[8] S. Kim, J. Chen, T. Cheng, A. Gindulyte, J. He, S. He, Q. Li, B. A. Shoemaker, P. A. Thiessen, B. Yu, L. Zaslavsky, J. Zhang, and E. E. Bolton, "PubChem 2019 update: improved access to chemical data," Nucleic Acids Res. 47 no. D1, (Jan, 2019) D1102-D1109. xxvii, 47, 113

[9] L. Geris, "Regenerative orthopaedics: in vitro, in vivo...in silico," Int Orthop $\mathbf{3 8}$ no. 9, (Sep, 2014) 1771-1778. 1, 4

[10] A. Mardinoglu and J. Nielsen, "Systems medicine and metabolic modelling," $J$. Intern. Med. 271 no. 2, (Feb, 2012) 142-154. 1

[11] A. Karolak, D. A. Markov, L. J. McCawley, and K. A. Rejniak, "Towards personalized computational oncology: from spatial models of tumour spheroids, to organoids, to tissues," J R Soc Interface 15 no. 138, (Jan, 2018) · 1, 41

[12] V. Andasari, A. Gerisch, G. Lolas, A. P. South, and M. A. Chaplain, "Mathematical modeling of cancer cell invasion of tissue: biological insight from mathematical analysis and computational simulation," J. Math. Biol. 63 no. 1, (Jul, 2011) 141-171. 2

[13] S. Benzekry, C. Lamont, A. Beheshti, A. Tracz, J. M. Ebos, L. Hlatky, and P. Hahnfeldt, "Classical mathematical models for description and prediction of experimental tumor growth," PLoS Comput. Biol. 10 no. 8, (Aug, 2014) e1003800.

[14] H. M. Byrne and M. A. Chaplain, "Growth of necrotic tumors in the presence and absence of inhibitors," Math. Biosci. 135 no. 2, (Jul, 1996) 187-216.

[15] A. Gerisch and M. A. Chaplain, "Mathematical modelling of cancer cell invasion of tissue: local and non-local models and the effect of adhesion," J. Theor. Biol. 250 no. 4, (Feb, 2008) 684-704.

[16] I. Ramis-Conde, M. A. Chaplain, A. R. Anderson, and D. Drasdo, "Multi-scale modelling of cancer cell intravasation: the role of cadherins in metastasis," Phys. Biol. 6 no. 1, (2009) 016008. 
[17] I. Ramis-Conde, D. Drasdo, A. R. Anderson, and M. A. Chaplain, "Modeling the influence of the E-cadherin-beta-catenin pathway in cancer cell invasion: a multiscale approach," Biophys. J. 95 no. 1, (Jul, 2008) 155-165. 2

[18] A. R. Anderson and M. A. Chaplain, "Continuous and discrete mathematical models of tumor-induced angiogenesis," Bull. Math. Biol. 60 (Sep, 1998) 857-899. 2

[19] P. Macklin, S. McDougall, A. R. A. Anderson, M. A. J. Chaplain, V. Cristini, and J. Lowengrub, "Multiscale modelling and nonlinear simulation of vascular tumour growth," J Math Biol 58 (Apr, 2009) 765-798. 42, 43

[20] S. R. McDougall, A. R. Anderson, and M. A. Chaplain, "Mathematical modelling of dynamic adaptive tumour-induced angiogenesis: clinical implications and therapeutic targeting strategies," J. Theor. Biol. 241 no. 3, (Aug, 2006) 564-589.

[21] T. Suzuki, D. Minerva, K. Nishiyama, N. Koshikawa, and M. A. J. Chaplain, "Study on the tumor-induced angiogenesis using mathematical models," Cancer Sci. 109 no. 1, (Jan, 2018) 15-23. 2

[22] Z. Agur, R. Hassin, and S. Levy, "Optimizing chemotherapy scheduling using local search heuristics," Operations Research 54 no. 5, (Sep-Oct, 2006) 829-846. 2

[23] H. Enderling, M. A. Chaplain, and P. Hahnfeldt, "Quantitative modeling of tumor dynamics and radiotherapy," Acta Biotheor. 58 no. 4, (Dec, 2010) 341-353. 34

[24] K. Fister and J. Panetta, "Optimal control applied to cell-cycle-specific cancer chemotherapy," SIAM Journal on Applied Mathematics (2000) 1059-1072.

[25] K. Leder, K. Pitter, Q. LaPlant, D. Hambardzumyan, B. D. Ross, T. A. Chan, E. C. Holland, and F. Michor, "Mathematical modeling of PDGF-driven glioblastoma reveals optimized radiation dosing schedules," Cell 156 no. 3, (Jan, 2014) 603-616. 


\section{REFERENCES}

[26] B. Ribba, T. Colin, and S. Schnell, "A multiscale mathematical model of cancer, and its use in analyzing irradiation therapies," Theor Biol Med Model 3 (2006) 7.

[27] B. Ribba, K. Marron, Z. Agur, T. Alarcon, and P. K. Maini, "A mathematical model of Doxorubicin treatment efficacy for non-Hodgkin's lymphoma: investigation of the current protocol through theoretical modelling results," Bull. Math. Biol. 67 no. 1, (Jan, 2005) 79-99.

[28] R. Rockne, E. Alvord, M. Szeto, S. Gu, G. Chakraborty, and K. Swanson, "Modeling diffusely invading brain tumors an individualized approach to quantifying glioma evolution and response to therapy," Selected Topics in Cancer Modeling (2008) 1-15. 2

[29] J. S. Lowengrub, H. B. Frieboes, F. Jin, Y. L. Chuang, X. Li, P. Macklin, S. M. Wise, and V. Cristini, "Nonlinear modelling of cancer: bridging the gap between cells and tumours," Nonlinearity 23 (2010) R1-R9. 2

[30] F. Billy, B. Ribba, O. Saut, H. Morre-Trouilhet, T. Colin, D. Bresch, J. P. Boissel, E. Grenier, and J. P. Flandrois, "A pharmacologically based multiscale mathematical model of angiogenesis and its use in investigating the efficacy of a new cancer treatment strategy," J. Theor. Biol. 260 no. 4, (Oct, 2009) 545-562. 2

[31] S. Bruningk, G. Powathil, P. Ziegenhein, J. Ijaz, I. Rivens, S. Nill, M. Chaplain, U. Oelfke, and G. Ter Haar, "Combining radiation with hyperthermia: a multiscale model informed by in vitro experiments," J R Soc Interface $\mathbf{1 5}$ no. 138, (Jan, 2018) . 67,99

[32] H. M. Byrne, "Dissecting cancer through mathematics: from the cell to the animal model," Nat. Rev. Cancer 10 (Mar, 2010) 221-230. 2

[33] S. E. Jackson and J. D. Chester, "Personalised cancer medicine," Int. J. Cancer 137 no. 2, (Jul, 2015) 262-266. 2, 3

[34] M. Lawler and R. Sullivan, "Personalised and Precision Medicine in Cancer Clinical Trials: Panacea for Progress or Pandora's Box?," Public Health Genomics 18 no. 6, (2015) 329-337. 2 
[35] C. Roussel and C. Touboul, "Large population survey: strengths and limits. Methodology of the EDIFICE survey," Eur. J. Cancer Prev. 20 Suppl 1 (Jan, 2011) 5-7. 2

[36] C. Dumontet, S. Couray-Targe, M. Teisseire, L. Karlin, and D. Maucort-Boulch, "Real life management of patients hospitalized with multiple myeloma in France," PLoS ONE 13 no. 5, (2018) e0196596. 2

[37] A. K. Turnbull, "Personalized medicine in cancer: where are we today?," Future Oncol 11 no. 20, (2015) 2795-2798. 2

[38] W. Burke, S. Brown Trinidad, and N. A. Press, "Essential elements of personalized medicine," Urol. Oncol. 32 no. 2, (Feb, 2014) 193-197. 2, 3

[39] G. Moutel, N. Duchange, S. Darquy, S. de Montgolfier, F. Papin-Lefebvre, O. Jullian, J. Viguier, H. Sancho-Garnier, G. Moutel, P. Aiach, I. Callies, S. Darquy, S. De Montgolfier, N. Duchange, O. Ferrand, O. Jullian, A. Leplege, M. B. Orgerie, F. Papin-Lefebvre, H. Sancho-Garnier, and D. Stoppa-Lyonnet, "Women's participation in breast cancer screening in France-an ethical approach," BMC Med Ethics 15 (Aug, 2014) 64. 2

[40] A. Bredart, J. L. Kop, A. C. Antoniou, A. P. Cunningham, A. De Pauw, M. Tischkowitz, H. Ehrencrona, M. K. Schmidt, S. Dolbeault, K. Rhiem, D. F. Easton, P. Devilee, D. Stoppa-Lyonnet, and R. Schmutlzer, "Clinicians' use of breast cancer risk assessment tools according to their perceived importance of breast cancer risk factors: an international survey," J Community Genet (Mar, 2018) 2, 3

[41] K. Curtius, N. A. Wright, and T. A. Graham, "An evolutionary perspective on field cancerization," Nat. Rev. Cancer 18 no. 1, (Jan, 2018) 19-32. 2, 3

[42] M. N. Duong, A. Geneste, F. Fallone, X. Li, C. Dumontet, and C. Muller, "The fat and the bad: Mature adipocytes, key actors in tumor progression and resistance," Oncotarget 8 no. 34, (Aug, 2017) 57622-57641. 3

[43] Z. Hu, P. A. Ott, and C. J. Wu, "Towards personalized, tumour-specific, therapeutic vaccines for cancer," Nat. Rev. Immunol. 18 no. 3, (Mar, 2018) 168-182. 3 


\section{REFERENCES}

[44] M. Tremblay-Servier, "Personalized medicine: the medicine of tomorrow. Foreword," Metab. Clin. Exp. 62 Suppl 1 (Jan, 2013) S1. 3

[45] N. Buil-Bruna, T. Sahota, J. M. Lopez-Picazo, M. Moreno-Jimenez, S. Martin-Algarra, B. Ribba, and I. F. Troconiz, "Early Prediction of Disease Progression in Small Cell Lung Cancer: Toward Model-Based Personalized Medicine in Oncology," Cancer Res. 75 no. 12, (Jun, 2015) 2416-2425. 3

[46] L. Gay, A. M. Baker, and T. A. Graham, "Tumour Cell Heterogeneity," F1000Res 5 (2016) . 3, 7

[47] T. E. Yankeelov, G. An, O. Saut, E. G. Luebeck, A. S. Popel, B. Ribba, P. Vicini, X. Zhou, J. A. Weis, K. Ye, and G. M. Genin, "Multi-scale Modeling in Clinical Oncology: Opportunities and Barriers to Success," Ann Biomed Eng 44 no. 9, (09, 2016) 2626-2641. 3

[48] K. A. Rejniak, M. C. Lloyd, D. R. Reed, and M. M. Bui, "Diagnostic assessment of osteosarcoma chemoresistance based on Virtual Clinical Trials," Med.

Hypotheses 85 no. 3, (Sep, 2015) 348-354. 3

[49] M. Lawler, P. Selby, and J. C. Wright, "Personalized cancer medicine: are we there yet?," Oncologist 18 no. 6, (Jun, 2013) 649-650. 5, 174

[50] K. A. Rejniak and A. R. Anderson, "Hybrid models of tumor growth," Wiley Interdiscip Rev Syst Biol Med 3 (2011) 115-125. 5, 6, 42, 105

[51] O. Wolkenhauer, C. Auffray, O. Brass, J. Clairambault, A. Deutsch, D. Drasdo, F. Gervasio, L. Preziosi, P. Maini, A. Marciniak-Czochra, C. Kossow, L. Kuepfer, K. Rateitschak, I. Ramis-Conde, B. Ribba, A. Schuppert, R. Smallwood, G. Stamatakos, F. Winter, and H. Byrne, "Enabling multiscale modeling in systems medicine," Genome Med 6 no. 3, (2014) 21. 5, 99, 125

[52] M. Soheilypour and M. R. K. Mofrad, "Agent-Based Modeling in Molecular Systems Biology," Bioessays 40 no. 7, (07, 2018) e1800020. 6

[53] P. Valent, D. Bonnet, S. Wohrer, M. Andreeff, M. Copland, C. Chomienne, and C. Eaves, "Heterogeneity of neoplastic stem cells: theoretical, functional, and clinical implications," Cancer Res. 73 no. 3, (Feb, 2013) 1037-1045. 6, 7 
[54] S. Rodic and M. D. Vincent, "Reactive oxygen species (ROS) are a key determinant of cancer's metabolic phenotype," Int. J. Cancer 142 no. 3, (02, 2018) 440-448. 6

[55] T. Alarcon, H. M. Byrne, and P. K. Maini, "A multiple scale model for tumour growth," Multiscale Model. Sim. 3 (2005) 440-475. 7

[56] T. S. Deisboeck, Z. Wang, P. Macklin, and V. Cristini, "Multiscale cancer modeling," Annu Rev Biomed Eng 13 (Aug, 2011) 127-155. 42

[57] L. Zhang, Z. Wang, J. A. Sagotsky, and T. S. Deisboeck, "Multiscale agent-based cancer modeling," J Math Biol 58 no. 4-5, (Apr, 2009) 545-559. 7, 42

[58] L. Preziosi and A. Tosin, "Multiphase modelling of tumour growth and extracellular matrix interaction: mathematical tools and applications," J Math Biol 58 no. 4-5, (Apr, 2009) 625-656. 9, 105

[59] H. Harashima, N. Dissmeyer, and A. Schnittger, "Cell cycle control across the eukaryotic kingdom," Trends Cell Biol. 23 no. 7, (Jul, 2013) 345-356. 10

[60] J. J. Tyson and B. Novak, "Regulation of the eukaryotic cell cycle: molecular antagonism, hysteresis, and irreversible transitions," J. Theor. Biol. 210 no. 2, (May, 2001) 249-263. 12, 13, 16, 46, 110

[61] B. Novak and J. J. Tyson, "Modelling the controls of the eukaryotic cell cycle," Biochem. Soc. Trans. 31 no. Pt 6, (Dec, 2003) 1526-1529.

[62] B. Novak and J. J. Tyson, "A model for restriction point control of the mammalian cell cycle," J. Theor. Biol. 230 no. 4, (Oct, 2004) 563-579. 13, 110

[63] G. Cooper and R. Hausman, "The Cell-A Molecular Approach 4th edition,". 17. 77, 108

[64] K. Alden, M. Read, J. Timmis, P. S. Andrews, H. Veiga-Fernandes, and M. Coles, "Spartan: a comprehensive tool for understanding uncertainty in simulations of biological systems," PLoS Comput. Biol. 9 no. 2, (2013) e1002916. $18,114,127,128,129,133,136,137,145,147$ 


\section{REFERENCES}

[65] R. M. Phillips, "Targeting the hypoxic fraction of tumours using hypoxia-activated prodrugs," Cancer Chemother. Pharmacol. 77 no. 3, (Mar, 2016) 441-457. 20, 72, 73

[66] C. T. Lee, M. K. Boss, and M. W. Dewhirst, "Imaging tumor hypoxia to advance radiation oncology," Antioxid. Redox Signal. 21 no. 2, (Jul, 2014) 313-337. 20, 72, 73

[67] G. Powathil, M. Kohandel, M. Milosevic, and S. Sivaloganathan, "Modeling the spatial distribution of chronic tumor hypoxia: implications for experimental and clinical studies," Comput Math Methods Med 2012 (2012) 410602. 21, 31, 46, 80, 110, 177

[68] G. M. Currie, "Pharmacology, Part 1: Introduction to Pharmacology and Pharmacodynamics," J Nucl Med Technol 46 no. 2, (Jun, 2018) 81-86. 22, 23, 24

[69] G. M. Currie, "Pharmacology, Part 2: Introduction to Pharmacokinetics," J Nucl Med Technol 46 no. 3, (Sep, 2018) 221-230. 22, 27

[70] N. Holford, "Pharmacodynamic principles and the time course of immediate drug effects. ," Transl Clin Pharmacol 4 (2017) 157-161. 24, 25, 26, 113,118

[71] M. S. Salahudeen and P. S. Nishtala, "An overview of pharmacodynamic modelling, ligand-binding approach and its application in clinical practice," Saudi Pharm J 25 no. 2, (Feb, 2017) 165-175.

[72] G. Alvan, G. Paintaud, and M. Wakelkamp, "The efficiency concept in pharmacodynamics," Clin Pharmacokinet 36 no. 5, (May, 1999) 375-389. 25

[73] M. A. Felmlee, M. E. Morris, and D. E. Mager, "Mechanism-based pharmacodynamic modeling," Methods Mol. Biol. 929 (2012) 583-600. 26

[74] M. A. Dickson and G. K. Schwartz, "Development of cell-cycle inhibitors for cancer therapy," Curr Oncol 16 no. 2, (Mar, 2009) 36-43. 26

[75] J. Bai, Y. Li, and G. Zhang, "Cell cycle regulation and anticancer drug discovery," Cancer Biol Med 14 no. 4, (Nov, 2017) 348-362. 26 
[76] V. Schirrmacher, "From chemotherapy to biological therapy: A review of novel concepts to reduce the side effects of systemic cancer treatment (Review)," Int. J. Oncol. 54 no. 2, (Feb, 2019) 407-419. 26

[77] W. Zhou, Y. Wang, A. Lu, and G. Zhang, "Systems Pharmacology in Small Molecular Drug Discovery," Int J Mol Sci 17 no. 2, (Feb, 2016) 246. 27

[78] M. Orth, K. Lauber, M. Niyazi, A. A. Friedl, M. Li, C. Maihofer, L. Schuttrumpf, A. Ernst, O. M. Niemoller, and C. Belka, "Current concepts in clinical radiation oncology," Radiat Environ Biophys 53 no. 1, (Mar, 2014) 1-29. 28

[79] E. Hall and A. Giaccia, "Radiobiology for the Radiologist,". 28, 29, 30, 31, 72, 77. 87

[80] J. G. Bazan, Q.-T. Le, and D. Zips, “35 - radiobiology of lung cancer," in IASLC Thoracic Oncology (Second Edition), H. I. Pass, D. Ball, and G. V. Scagliotti, eds., pp. 330 - 336.e2. Philadelphia, second edition ed., 2018. 28

[81] O. Desouky, N. Din, and G. Zhou, "Targeted and non-targeted effects of ionizing radiation," Journal of Radiation Research and Applied Sciences 67 (03, 2015). 28

[82] A. D. Horan, A. R. Giandomenico, and C. J. Koch, "Effect of oxygen on radiation-induced DNA damage in isolated nuclei," Radiat. Res. 152 no. 2, (Aug, 1999) 144-153. 29, 30

[83] J. C. Forster, M. J. J. Douglass, W. M. Phillips, and E. Bezak, "Monte Carlo Simulation of the Oxygen Effect in DNA Damage Induction by Ionizing Radiation," Radiat. Res. 190 no. 3, (09, 2018) 248-261. 29

[84] D. R. Grimes and M. Partridge, "A mechanistic investigation of the oxygen fixation hypothesis and oxygen enhancement ratio," Biomed Phys Eng Express 1 no. 4, (Dec, 2015) 045209. 30

[85] N. Baran and M. Konopleva, "Molecular Pathways: Hypoxia-Activated Prodrugs in Cancer Therapy," Clin. Cancer Res. 23 no. 10, (May, 2017) 2382-2390. 30. 72, 73, 74, 75 


\section{REFERENCES}

[86] S. J. McMahon, "The linear quadratic model: usage, interpretation and challenges," Phys Med Biol 64 no. 1, (12, 2018) 01TR01. 31, 32, 33, 34

[87] W. C. Rontgen, "ON A NEW KIND OF RAYS," Science 3 no. 59, (Feb, 1896) 227-231. 32

[88] M. R. Horsman and J. Overgaard, "The impact of hypoxia and its modification of the outcome of radiotherapy," J. Radiat. Res. 57 Suppl 1 (Aug, 2016) i90-i98. 34, 72, 85

[89] T. M. Pawlik and K. Keyomarsi, "Role of cell cycle in mediating sensitivity to radiotherapy," Int. J. Radiat. Oncol. Biol. Phys. 59 no. 4, (Jul, 2004) 928-942. 34, 85

[90] W. K. Sinclair, "Cyclic X-ray responses in mammalian cells in vitro. 1968," Radiat. Res. 178 no. 2, (Aug, 2012) V112-124. 34.85

[91] G. G. Powathil, M. Swat, and M. A. Chaplain, "Systems oncology: towards patient-specific treatment regimes informed by multiscale mathematical modelling," Semin. Cancer Biol. 30 (Feb, 2015) 13-20. 34

[92] K. Abubaker, A. Latifi, R. Luwor, S. Nazaretian, H. Zhu, M. A. Quinn, E. W. Thompson, J. K. Findlay, and N. Ahmed, "Short-term single treatment of chemotherapy results in the enrichment of ovarian cancer stem cell-like cells leading to an increased tumor burden," Mol. Cancer 12 (Mar, 2013) 24. 38, 40

[93] J. Foo and F. Michor, "Evolution of acquired resistance to anti-cancer therapy," J. Theor. Biol. 355 (Aug, 2014) 10-20. 39, 40, 60

[94] H. Guo, J. Dong, S. Hu, X. Cai, G. Tang, J. Dou, M. Tian, F. He, Y. Nie, and D. Fan, "Biased random walk model for the prioritization of drug resistance associated proteins," Sci Rep 5 (Jun, 2015) 10857.

[95] O. Lavi, M. M. Gottesman, and D. Levy, "The dynamics of drug resistance: a mathematical perspective," Drug Resist. Updat. 15 no. 1-2, (2012) 90-97. 38, 39, 41, 49, 50, 58

[96] N. Moore, J. Houghton, and S. Lyle, "Slow-cycling therapy-resistant cancer cells," Stem Cells Dev. 21 no. 10, (Jul, 2012) 1822-1830. 40, 53 
[97] E. L. Niero, B. Rocha-Sales, C. Lauand, B. A. Cortez, M. M. de Souza, P. Rezende-Teixeira, M. S. Urabayashi, A. A. Martens, J. H. Neves, and G. M. Machado-Santelli, "The multiple facets of drug resistance: one history, different approaches," J. Exp. Clin. Cancer Res. 33 (Apr, 2014) 37.

[98] S. Rottenberg and P. Borst, "Drug resistance in the mouse cancer clinic," Drug Resist. Updat. 15 no. 1-2, (2012) 81-89. 38, 59

[99] S. Oskay Halacli, B. Halacli, and K. Altundag, "The significance of heat shock proteins in breast cancer therapy," Med. Oncol. 30 no. 2, (2013) 575. 38, 40, 51

[100] S. Rizzo, J. M. Hersey, P. Mellor, W. Dai, A. Santos-Silva, D. Liber, L. Luk, I. Titley, C. P. Carden, G. Box, D. L. Hudson, S. B. Kaye, and R. Brown, "Ovarian cancer stem cell-like side populations are enriched following chemotherapy and overexpress EZH2," Mol. Cancer Ther. 10 no. 2, (Feb, 2011) 325-335. 40

[101] L. M. Vargas-Roig, F. E. Gago, O. Tello, J. C. Aznar, and D. R. Ciocca, "Heat shock protein expression and drug resistance in breast cancer patients treated with induction chemotherapy," Int. J. Cancer 79 no. 5, (Oct, 1998) 468-475. 38 , 40, 51, 52

[102] G. Duan, Q. Tang, H. Yan, L. Xie, Y. Wang, X. E. Zheng, Y. Zhuge, S. Shen, B. Zhang, X. Zhang, J. Wang, W. Wang, and X. Zou, "A Strategy to Delay the Development of Cisplatin Resistance by Maintaining a Certain Amount of Cisplatin-Sensitive Cells," Sci Rep 7 no. 1, (Mar, 2017) 432. 38, 39

[103] J. D. Sun, Q. Liu, D. Ahluwalia, D. J. Ferraro, Y. Wang, D. Jung, M. D. Matteucci, and C. P. Hart, "Comparison of hypoxia-activated prodrug evofosfamide (TH-302) and ifosfamide in preclinical non-small cell lung cancer models," Cancer Biol. Ther. 17 no. 4, (Apr, 2016) 371-380. 41, 45, 73, 74, 80

[104] A. Wu, K. Loutherback, G. Lambert, L. Estevez-Salmeron, T. D. Tlsty, R. H. Austin, and J. C. Sturm, "Cell motility and drug gradients in the emergence of resistance to chemotherapy," Proc. Natl. Acad. Sci. U.S.A. 110 no. 40, (Oct, 2013) 16103-16108. 38 


\section{REFERENCES}

[105] D. P. Tabassum and K. Polyak, "Tumorigenesis: it takes a village," Nat. Rev. Cancer 15 no. 8, (Aug, 2015) 473-483. 38, 39, 41, 45

[106] H. C. Monro and E. A. Gaffney, "Modelling chemotherapy resistance in palliation and failed cure," J. Theor. Biol. 257 no. 2, (Mar, 2009) 292-302. 38. 39, 41, 43, 58

[107] M. M. Hadjiandreou and G. D. Mitsis, "Mathematical modeling of tumor growth, drug-resistance, toxicity, and optimal therapy design," IEEE Trans Biomed Eng 61 no. 2, (Feb, 2014) 415-425. 38, 41

[108] C. Munck, H. K. Gumpert, A. I. Wallin, H. H. Wang, and M. O. Sommer, "Prediction of resistance development against drug combinations by collateral responses to component drugs," Sci Transl Med 6 no. 262, (Nov, 2014) 262ra156. 38,39

[109] S. Srinivasan, F. O. Vannberg, and J. B. Dixon, "Lymphatic transport of exosomes as a rapid route of information dissemination to the lymph node," Sci Rep 6 (Apr, 2016) 24436. 38, 40, 41, 49, 52, 53

[110] T. Srinivasan, J. Walters, P. Bu, E. B. Than, K. L. Tung, K. Y. Chen, N. Panarelli, J. Milsom, L. Augenlicht, S. M. Lipkin, and X. Shen, "NOTCH Signaling Regulates Asymmetric Cell Fate of Fast- and Slow-Cycling Colon Cancer-Initiating Cells," Cancer Res. 76 no. 11, (Jun, 2016) 3411-3421. 39, 40. 53

[111] A. W. Park, J. Haven, R. Kaplan, and S. Gandon, "Refugia and the evolutionary epidemiology of drug resistance," Biol. Lett. 11 no. 11, (Nov, 2015) . 39

[112] N. A. Saunders, F. Simpson, E. W. Thompson, M. M. Hill, L. Endo-Munoz, G. Leggatt, R. F. Minchin, and A. Guminski, "Role of intratumoural heterogeneity in cancer drug resistance: molecular and clinical perspectives," EMBO Mol Med 4 no. 8, (Aug, 2012) 675-684. 39, 45, 49

[113] H. B. Frieboes, B. R. Smith, Z. Wang, M. Kotsuma, K. Ito, A. Day, B. Cahill, C. Flinders, S. M. Mumenthaler, P. Mallick, E. Simbawa, A. S. Al-Fhaid, S. R. Mahmoud, S. S. Gambhir, and V. Cristini, "Predictive Modeling of Drug 
Response in Non-Hodgkin's Lymphoma," PLoS ONE 10 no. 6, (2015) e0129433. 39

[114] Z. Kozovska, V. Gabrisova, and L. Kucerova, "Colon cancer: cancer stem cells markers, drug resistance and treatment," Biomed. Pharmacother. 68 no. 8, (Oct, 2014) 911-916. 39

[115] S. E. Luria and M. Delbruck, "Mutations of Bacteria from Virus Sensitivity to Virus Resistance," Genetics 28 no. 6, (Nov, 1943) 491-511. 39, 50, 54, 58

[116] G. Tzedakis, E. Liapis, E. Tzamali, G. Zacharakis, and V. Sakkalis, "A hybrid discrete-continuous model of in vitro spheroid tumor growth and drug response," Conf Proc IEEE Eng Med Biol Soc 2016 (Aug, 2016) 6142-6145. 40, 51

[117] G. Jego, A. Hazoume, R. Seigneuric, and C. Garrido, "Targeting heat shock proteins in cancer," Cancer Lett. 332 no. 2, (May, 2013) 275-285. 40, 51

[118] S. P. Shah, S. Lonial, and L. H. Boise, "When Cancer Fights Back: Multiple Myeloma, Proteasome Inhibition, and the Heat-Shock Response," Mol. Cancer Res. 13 no. 8, (Aug, 2015) 1163-1173. 40, 51

[119] K. Chen, W. Cao, J. Li, D. Sprengers, P. Y. Hernanda, X. Kong, L. J. Van Der Laan, K. Man, J. Kwekkeboom, H. J. Metselaar, M. P. Peppelenbosch, and Q. Pan, "Differential Sensitivities of Fast- and Slow-cycling Cancer Cells to Inosine Monophosphate Dehydrogenase 2 Inhibition by Mycophenolic Acid," Mol. Med. (Oct, 2015) . 40, 53

[120] X. Zhang, X. Yuan, H. Shi, L. Wu, H. Qian, and W. Xu, "Exosomes in cancer: small particle, big player," J Hematol Oncol 8 (Jul, 2015) 83. 40, 41, 49, 52

[121] V. D. Dang, K. K. Jella, R. R. T. Ragheb, N. D. Denslow, and A. A. Alli, "Lipidomic and proteomic analysis of exosomes from mouse cortical collecting duct cells," FASEB J. 31 no. 12, (12, 2017) 5399-5408. 41

[122] K. K. Jella, L. Yu, Q. Yue, D. Friedman, B. J. Duke, and A. A. Alli, "Exosomal GAPDH from Proximal Tubule Cells Regulate ENaC Activity," PLoS ONE 11 no. 11, (2016) e0165763. 41 


\section{REFERENCES}

[123] G. Cesi, G. Walbrecq, C. Margue, and S. Kreis, "Transferring intercellular signals and traits between cancer cells: extracellular vesicles as "homing pigeons"," Cell Commun. Signal 14 no. 1, (Jun, 2016) 13. 41, 49, 52, 53

[124] L. Gangoda, S. Boukouris, M. Liem, H. Kalra, and S. Mathivanan, "Extracellular vesicles including exosomes are mediators of signal transduction: are they protective or pathogenic?," Proteomics 15 no. 2-3, (Jan, 2015) 260-271. 41

[125] H. Hatzikirou, A. Chauviere, A. L. Bauer, A. Leier, M. T. Lewis, P. Macklin, T. T. Marquez-Lago, E. L. Bearer, and V. Cristini, "Integrative physical oncology," Wiley Interdiscip Rev Syst Biol Med 4 no. 1, (2012) 1-14. 41

[126] N. Glade and A. Stephanou, "XXXIVth Seminar of the French-Speaking Society for Theoretical Biology: Saint-Flour (Cantal), France, 26-28 May, 2014," Acta Biotheor. 63 no. 3, (Sep, 2015) 237-238. 41

[127] M. Viceconti, "Biomechanics-based in silico medicine: the manifesto of a new science," J Biomech 48 no. 2, (Jan, 2015) 193-194. 41

[128] N. Bellomo, N. Li, and P. K. Maini, "On the foundations of cancer modelling: selected topics, speculations, and perspectives," Mathematical Models and Methods in Applied Sciences 18 no. 04, (2008) 593-646. 41

[129] A. Lorz, D. A. Botesteanu, and D. Levy, "Modeling Cancer Cell Growth Dynamics In vitro in Response to Antimitotic Drug Treatment," Front Oncol 7 (2017) 189. 43

[130] J. C. Panetta, "A mathematical model of periodically pulsed chemotherapy: tumor recurrence and metastasis in a competitive environment," Bulletin of mathematical Biology 58 no. 3, (1996) 425-447. 43

[131] J. Perez-Velazquez, J. L. Gevertz, A. Karolak, and K. A. Rejniak, "Microenvironmental Niches and Sanctuaries: A Route to Acquired Resistance," Adv. Exp. Med. Biol. 936 (2016) 149-164. 43

[132] G. G. Powathil, M. A. Chaplain, and M. Swat, "Investigating the development of chemotherapeutic drug resistance in cancer: A multiscale computational study," arXiv preprint arXiv:1407.0865 (2014) . 42, 54 
[133] T. Roose, S. J. Chapman, and P. K. Maini, "Mathematical models of avascular tumor growth," Siam Review 49 no. 2, (2007) 179-208. 41

[134] A. Stéphanou and V. Volpert, "Hybrid modelling in biology: a classification review," Mathematical Modelling of Natural Phenomena 11 no. 1, (2016) 37-48. 41

[135] M. H. Swat, G. L. Thomas, J. M. Belmonte, A. Shirinifard, D. Hmeljak, and J. A. Glazier, "Multi-scale modeling of tissues using CompuCell3D," Methods Cell Biol. 110 (2012) 325-366. 42

[136] T. Alarcon, H. M. Byrne, and P. K. Maini, "A cellular automaton model for tumour growth in inhomogeneous environment," J. Theor. Biol. 225 (Nov, 2003) 257-274. 42

[137] A. R. Anderson, K. A. Rejniak, P. Gerlee, and V. Quaranta, "Microenvironment driven invasion: a multiscale multimodel investigation," J Math Biol 58 (Apr, 2009) 579-624.

[138] P. Gerlee and A. R. Anderson, "An evolutionary hybrid cellular automaton model of solid tumour growth," J. Theor. Biol. 246 (Jun, 2007) 583-603.

[139] T. L. Jackson and H. M. Byrne, "A mathematical model to study the effects of drug resistance and vasculature on the response of solid tumors to chemotherapy," Mathematical biosciences 164 no. 1, (2000) 17-38.

[140] A. R. Kansal, S. Torquato, G. R. Harsh IV, E. A. Chiocca, and T. S. Deisboeck, "Cellular automaton of idealized brain tumor growth dynamics," BioSystems $\mathbf{5 5}$ (Feb, 2000) 119-127.

[141] A. A. Patel, E. T. Gawlinski, S. K. Lemieux, and R. A. Gatenby, "A cellular automaton model of early tumor growth and invasion," J. Theor. Biol. 213 (Dec, 2001) 315-331.

[142] B. Ribba, T. Alarcon, K. Marron, P. Maini, and Z. Agur, "The Use of Hybrid Cellular Automaton Models for Improving Cancer Therapy," Lect. notes comput. sci 3305 (2004) 444-453. 42 


\section{REFERENCES}

[143] M. R. Owen, T. Alarcon, P. K. Maini, and H. M. Byrne, "Angiogenesis and vascular remodelling in normal and cancerous tissues," J Math Biol 58 (Apr, 2009) 689-721. 42, 43

[144] H. Perfahl, H. M. Byrne, T. Chen, V. Estrella, T. Alarcon, A. Lapin, R. A. Gatenby, R. J. Gillies, M. C. Lloyd, P. K. Maini, M. Reuss, and M. R. Owen, "Multiscale modelling of vascular tumour growth in 3D: the roles of domain size and boundary conditions," PLoS ONE 6 (2011) e14790. 42, 43

[145] G. G. Powathil, D. J. Adamson, and M. A. Chaplain, "Towards predicting the response of a solid tumour to chemotherapy and radiotherapy treatments: clinical insights from a computational model," PLoS Comput. Biol. 9 no. 7, (Jul, 2013) e1003120. 42

[146] F. Caraguel, A. C. Lesart, F. Esteve, B. van der Sanden, and A. Stephanou, "Towards the Design of a Patient-Specific Virtual Tumour," Comput Math Methods Med 2016 (2016) 7851789. 42, 43

[147] G. G. Powathil, A. J. Munro, M. A. Chaplain, and M. Swat, "Bystander effects and their implications for clinical radiation therapy: Insights from multiscale in silico experiments," J. Theor. Biol. 401 (Jul, 2016) 1-14. 43

[148] F. Un, "G1 arrest induction represents a critical determinant for cisplatin cytotoxicity in G1 checkpoint-retaining human cancers," Anticancer Drugs $\mathbf{1 8}$ no. 4, (Apr, 2007) 411-417. 46, 53

[149] A. Carreau, B. El Hafny-Rahbi, A. Matejuk, C. Grillon, and C. Kieda, "Why is the partial oxygen pressure of human tissues a crucial parameter? Small molecules and hypoxia," J. Cell. Mol. Med. 15 no. 6, (Jun, 2011) 1239-1253. 46

[150] M. R. Owen, H. M. Byrne, and C. E. Lewis, "Mathematical modelling of the use of macrophages as vehicles for drug delivery to hypoxic tumour sites," J. Theor. Biol. 226 no. 4, (Feb, 2004) 377-391. 47

[151] A. Matzavinos, C. Y. Kao, J. E. Green, A. Sutradhar, M. Miller, and A. Friedman, "Modeling oxygen transport in surgical tissue transfer," Proc. Natl. Acad. Sci. U.S.A. 106 no. 29, (Jul, 2009) 12091-12096. 47 
[152] P. A. Reece, I. Stafford, M. Davy, and S. Freeman, "Disposition of unchanged cisplatin in patients with ovarian cancer," Clin. Pharmacol. Ther. 42 no. 3, (Sep, 1987) 320-325. 47, 48

[153] K. R. Kutanzi, O. V. Yurchenko, F. A. Beland, V. F. Checkhun, and I. P. Pogribny, "MicroRNA-mediated drug resistance in breast cancer," Clin Epigenetics 2 no. 2, (Aug, 2011) 171-185. 49, 51

[154] A. Roberti, D. La Sala, and C. Cinti, "Multiple genetic and epigenetic interacting mechanisms contribute to clonally selection of drug-resistant tumors: current views and new therapeutic prospective," J. Cell. Physiol. 207 no. 3, (Jun, 2006) 571-581. 49, 50, 51

[155] M. Gerlinger and C. Swanton, "How Darwinian models inform therapeutic failure initiated by clonal heterogeneity in cancer medicine," Br. J. Cancer 103 no. 8, (Oct, 2010) 1139-1143. 49

[156] B. Wang, C. W. Lee, A. Witt, A. Thakkar, and T. A. Ince, "Heat shock factor 1 induces cancer stem cell phenotype in breast cancer cell lines," Breast Cancer Res. Treat. 153 no. 1, (Aug, 2015) 57-66. 51, 52, 53

[157] M. Tong, W. Zheng, X. Lu, L. Ao, X. Li, Q. Guan, H. Cai, M. Li, H. Yan, Y. Guo, P. Chi, and Z. Guo, "Identifying clinically relevant drug resistance genes in drug-induced resistant cancer cell lines and post-chemotherapy tissues," Oncotarget 6 no. 38, (Dec, 2015) 41216-41227. 52

[158] J. Adamski, A. Price, C. Dive, and G. Makin, "Hypoxia-induced cytotoxic drug resistance in osteosarcoma is independent of HIF-1Alpha," PLoS ONE 8 no. 6, (2013) e65304. 52

[159] J. L. Dembinski and S. Krauss, "Characterization and functional analysis of a slow cycling stem cell-like subpopulation in pancreas adenocarcinoma," Clin. Exp. Metastasis 26 no. 7, (2009) 611-623. 53

[160] C. L. Chaffer, I. Brueckmann, C. Scheel, A. J. Kaestli, P. A. Wiggins, L. O. Rodrigues, M. Brooks, F. Reinhardt, Y. Su, K. Polyak, L. M. Arendt, C. Kuperwasser, B. Bierie, and R. A. Weinberg, "Normal and neoplastic 


\section{REFERENCES}

nonstem cells can spontaneously convert to a stem-like state," Proc. Natl. Acad. Sci. U.S.A. 108 no. 19, (May, 2011) 7950-7955. 53

[161] Ayachit and Utkarsh, "The ParaView Guide: A Parallel Visualization Application, ISBN: 978-1930934306," Kitware (2015) . 57, 85, 115, 177

[162] L. Spiegelberg, R. Houben, R. Niemans, D. de Ruysscher, A. Yaromina, J. Theys, C. P. Guise, J. B. Smaill, A. V. Patterson, P. Lambin, and L. J. Dubois, "Hypoxia-activated prodrugs and (lack of) clinical progress: The need for hypoxia-based biomarker patient selection in phase III clinical trials," Clin Transl Radiat Oncol 15 (Feb, 2019) 62-69. 71, 72, 73, 74, 75, 98

[163] F. W. Hunter, B. G. Wouters, and W. R. Wilson, "Hypoxia-activated prodrugs: paths forward in the era of personalised medicine," Br. J. Cancer 114 no. 10, (May, 2016) 1071-1077. 72, 74

[164] W. A. Denny, "Hypoxia-activated prodrugs in cancer therapy: progress to the clinic," Future Oncol 6 no. 3, (Mar, 2010) 419-428. 72, 73

[165] V. Liapis, A. Labrinidis, I. Zinonos, S. Hay, V. Ponomarev, V. Panagopoulos, M. DeNichilo, W. Ingman, G. J. Atkins, D. M. Findlay, A. C. Zannettino, and A. Evdokiou, "Hypoxia-activated pro-drug TH-302 exhibits potent tumor suppressive activity and cooperates with chemotherapy against osteosarcoma," Cancer Lett. 357 no. 1, (Feb, 2015) 160-169. 72, 74, 109

[166] Q. Liu, J. D. Sun, J. Wang, D. Ahluwalia, A. F. Baker, L. D. Cranmer, D. Ferraro, Y. Wang, J. X. Duan, W. S. Ammons, J. G. Curd, M. D. Matteucci, and C. P. Hart, "TH-302, a hypoxia-activated prodrug with broad in vivo preclinical combination therapy efficacy: optimization of dosing regimens and schedules," Cancer Chemother. Pharmacol. 69 no. 6, (Jun, 2012) 1487-1498. 72. 74

[167] I. N. Mistry, M. Thomas, E. D. D. Calder, S. J. Conway, and E. M. Hammond, "Clinical Advances of Hypoxia-Activated Prodrugs in Combination With Radiation Therapy," Int. J. Radiat. Oncol. Biol. Phys. 98 no. 5, (08, 2017) 1183-1196. 73, 74 
[168] S. G. Peeters, C. M. Zegers, R. Biemans, N. G. Lieuwes, R. G. van Stiphout, A. Yaromina, J. D. Sun, C. P. Hart, A. D. Windhorst, W. van Elmpt, L. J. Dubois, and P. Lambin, "TH-302 in Combination with Radiotherapy Enhances the Therapeutic Outcome and Is Associated with Pretreatment [18F]HX4 Hypoxia PET Imaging," Clin. Cancer Res. 21 no. 13, (Jul, 2015) 2984-2992. 72. 74, 109,110

[169] J. D. Sun, Q. Liu, J. Wang, D. Ahluwalia, D. Ferraro, Y. Wang, J. X. Duan, W. S. Ammons, J. G. Curd, M. D. Matteucci, and C. P. Hart, "Selective tumor hypoxia targeting by hypoxia-activated prodrug TH-302 inhibits tumor growth in preclinical models of cancer," Clin. Cancer Res. 18 no. 3, (Feb, 2012) 758-770. 74, 110

[170] G. J. Weiss, J. R. Infante, E. G. Chiorean, M. J. Borad, J. C. Bendell, J. R. Molina, R. Tibes, R. K. Ramanathan, K. Lewandowski, S. F. Jones, M. E. Lacouture, V. K. Langmuir, H. Lee, S. Kroll, and H. A. Burris, "Phase 1 study of the safety, tolerability, and pharmacokinetics of TH-302, a hypoxia-activated prodrug, in patients with advanced solid malignancies," Clin. Cancer Res. 17 no. 9, (May, 2011) 2997-3004. 72, 84

[171] J. Hu, D. R. Handisides, E. Van Valckenborgh, H. De Raeve, E. Menu, I. Vande Broek, Q. Liu, J. D. Sun, B. Van Camp, C. P. Hart, and K. Vanderkerken, "Targeting the multiple myeloma hypoxic niche with TH-302, a hypoxia-activated prodrug," Blood 116 no. 9, (Sep, 2010) 1524-1527. 72, 74. 109

[172] C. P. Guise, A. M. Mowday, A. Ashoorzadeh, R. Yuan, W. H. Lin, D. H. Wu, J. B. Smaill, A. V. Patterson, and K. Ding, "Bioreductive prodrugs as cancer therapeutics: targeting tumor hypoxia," Chin J Cancer 33 no. 2, (Feb, 2014) 80-86. 77, 73

[173] W. A. Denny, "The role of hypoxia-activated prodrugs in cancer therapy," Lancet Oncol. 1 no. 1, (Sep, 2000) 25-29. 72,73

[174] J. X. Duan, H. Jiao, J. Kaizerman, T. Stanton, J. W. Evans, L. Lan, G. Lorente, M. Banica, D. Jung, J. Wang, H. Ma, X. Li, Z. Yang, R. M. Hoffman, W. S. Ammons, C. P. Hart, and M. Matteucci, "Potent and highly selective 


\section{REFERENCES}

hypoxia-activated achiral phosphoramidate mustards as anticancer drugs," $J$. Med. Chem. 51 no. 8, (Apr, 2008) 2412-2420.

[175] F. Meng, J. W. Evans, D. Bhupathi, M. Banica, L. Lan, G. Lorente, J. X. Duan, X. Cai, A. M. Mowday, C. P. Guise, A. Maroz, R. F. Anderson, A. V. Patterson, G. C. Stachelek, P. M. Glazer, M. D. Matteucci, and C. P. Hart, "Molecular and cellular pharmacology of the hypoxia-activated prodrug TH-302," Mol. Cancer Ther. 11 no. 3, (Mar, 2012) 740-751. 72, 74, 76, 84

[176] C. R. Hong, B. D. Dickson, J. K. Jaiswal, F. B. Pruijn, F. W. Hunter, M. P. Hay, K. O. Hicks, and W. R. Wilson, "Cellular pharmacology of evofosfamide (TH-302): A critical re-evaluation of its bystander effects," Biochem. Pharmacol. $156(10,2018) 265-280.73,74$

[177] A. Foehrenbacher, T. W. Secomb, W. R. Wilson, and K. O. Hicks, "Design of optimized hypoxia-activated prodrugs using pharmacokinetic/pharmacodynamic modeling," Front Oncol 3 (Dec, 2013) 314. 73,74

[178] Y. Huang, Y. Tian, Y. Zhao, C. Xue, J. Zhan, L. Liu, X. He, and L. Zhang, "Efficacy of the hypoxia-activated prodrug evofosfamide (TH-302) in nasopharyngeal carcinoma in vitro and in vivo," Cancer Commun (Lond) $\mathbf{3 8}$ no. 1, (May, 2018) 15. 74

[179] D. Jung, H. Jiao, J. X. Duan, M. Matteucci, and R. Wang, "Metabolism, pharmacokinetics and excretion of a novel hypoxia activated cytotoxic prodrug, TH-302, in rats," Xenobiotica 42 no. 4, (Apr, 2012) 372-388.

[180] D. Jung, L. Lin, H. Jiao, X. Cai, J. X. Duan, and M. Matteucci, "Pharmacokinetics of TH-302: a hypoxically activated prodrug of bromo-isophosphoramide mustard in mice, rats, dogs and monkeys," Cancer Chemother. Pharmacol. 69 no. 3, (Mar, 2012) 643-654. 84

[181] K. J. Nytko, I. Grgic, S. Bender, J. Ott, M. Guckenberger, O. Riesterer, and M. Pruschy, "The hypoxia-activated prodrug evofosfamide in combination with multiple regimens of radiotherapy," Oncotarget 8 no. 14, (Apr, 2017) 23702-23712. 74 
[182] A. Yaromina, M. Granzier, R. Biemans, N. Lieuwes, W. van Elmpt, G. Shakirin, L. Dubois, and P. Lambin, "A novel concept for tumour targeting with radiation: Inverse dose-painting or targeting the "Low Drug Uptake Volume"," Radiother Oncol 124 no. 3, (09, 2017) 513-520. 74

[183] A. Foehrenbacher, K. Patel, M. R. Abbattista, C. P. Guise, T. W. Secomb, W. R. Wilson, and K. O. Hicks, "The Role of Bystander Effects in the Antitumor Activity of the Hypoxia-Activated Prodrug PR-104," Front Oncol 3 (2013) 263. 74

[184] D. Lindsay, C. M. Garvey, S. M. Mumenthaler, and J. Foo, "Leveraging Hypoxia-Activated Prodrugs to Prevent Drug Resistance in Solid Tumors," PLoS Comput. Biol. 12 no. 8, (Aug, 2016) e1005077. 74

[185] J. W. Wojtkowiak, H. C. Cornnell, S. Matsumoto, K. Saito, Y. Takakusagi, P. Dutta, M. Kim, X. Zhang, R. Leos, K. M. Bailey, G. Martinez, M. C. Lloyd, C. Weber, J. B. Mitchell, R. M. Lynch, A. F. Baker, R. A. Gatenby, K. A. Rejniak, C. Hart, M. C. Krishna, and R. J. Gillies, "Pyruvate sensitizes pancreatic tumors to hypoxia-activated prodrug TH-302," Cancer Metab 3 no. 1, (2015) 2. 75

[186] S. Hamis, G. G. Powathil, and M. A. J. Chaplain, "Blackboard to Bedside: A Mathematical Modeling Bottom-Up Approach Toward Personalized Cancer Treatments," JCO Clin Cancer Inform 3 (02, 2019) 1-11. 75, 76, 125, 174

[187] S. Hamis, P. Nithiarasu, and G. G. Powathil, "What does not kill a tumour may make it stronger: In silico insights into chemotherapeutic drug resistance," $J$. Theor. Biol. 454 (Jun, 2018) 253-267. 76, 125

[188] C. R. Hong, W. R. Wilson, and K. O. Hicks, "An Intratumor Pharmacokinetic/Pharmacodynamic Model for the Hypoxia-Activated Prodrug Evofosfamide (TH-302): Monotherapy Activity is Not Dependent on a Bystander Effect," Neoplasia 21 no. 2, (02, 2019) 159-171. 82

[189] C. Meaney, G. Powathil, A. Yaromina, L. Dubois, P. Lambin, and M. Kohandel, "Role of hypoxia-activated prodrugs in combination with radiation therapy: An in silico approach," Mathematical Biosciences and Engineering 16 (01, 2019) 6257-6273. 82 


\section{REFERENCES}

[190] C. Hajj, J. Russell, C. P. Hart, K. A. Goodman, M. A. Lowery, A. Haimovitz-Friedman, J. O. Deasy, and J. L. Humm, "A Combination of Radiation and the Hypoxia-Activated Prodrug Evofosfamide (TH-302) is Efficacious against a Human Orthotopic Pancreatic Tumor Model," Transl Oncol 10 no. 5, (Oct, 2017) 760-765. 84

[191] O. Tredan, A. B. Garbens, A. S. Lalani, and I. F. Tannock, "The hypoxia-activated ProDrug AQ4N penetrates deeply in tumor tissues and complements the limited distribution of mitoxantrone," Cancer Res. 69 no. 3, (Feb, 2009) 940-947. 84

[192] S. Hamis, J. Yates, M. Chaplain, and G. Powathil, "Targeting cellular dna damage responses: Predicting in vivo treatment responses using an in vitro-calibrated agent-based mathematical model," (To Appear) . 99

[193] J. Cosgrove, J. Butler, K. Alden, M. Read, V. Kumar, L. Cucurull-Sanchez, J. Timmis, and M. Coles, "Agent-Based Modeling in Systems Pharmacology," CPT Pharmacometrics Syst Pharmacol 4 no. 11, (Nov, 2015) 615-629. 102, 125

[194] A. Minchom, C. Aversa, and J. Lopez, "Dancing with the DNA damage response: next-generation anti-cancer therapeutic strategies," Ther Adv Med Oncol 10 (2018) 1758835918786658. 103, 104

[195] R. Sundar, J. Brown, A. Ingles Russo, and T. A. Yap, "Targeting ATR in cancer medicine," Curr Probl Cancer 41 no. 4, (2017) 302-315. 103, 104

[196] L. Carrassa and G. Damia, "DNA damage response inhibitors: Mechanisms and potential applications in cancer therapy," Cancer Treat. Rev. 60 (Nov, 2017) $139-151.103,104,106$

[197] A. R. Nam, M. H. Jin, J. E. Park, J. H. Bang, D. Y. Oh, and Y. J. Bang, "Therapeutic Targeting of the DNA Damage Response Using an ATR Inhibitor in Biliary Tract Cancer," Cancer Res Treat (Dec, 2018) . 103

[198] L. Mei, J. Zhang, K. He, and J. Zhang, "Ataxia telangiectasia and Rad3-related inhibitors and cancer therapy: where we stand," J Hematol Oncol 12 no. 1, (04, 2019) 43. 104 
[199] K. M. Foote, J. W. M. Nissink, T. McGuire, P. Turner, S. Guichard, J. W. T. Yates, A. Lau, K. Blades, D. Heathcote, R. Odedra, G. Wilkinson, Z. Wilson, C. M. Wood, and P. J. Jewsbury, "Discovery and Characterization of AZD6738, a Potent Inhibitor of Ataxia Telangiectasia Mutated and Rad3 Related (ATR) Kinase with Application as an Anticancer Agent," J. Med. Chem. 61 no. 22, (11, 2018) 9889-9907. 104

[200] X. Tu, M. M. Kahila, Q. Zhou, J. Yu, K. R. Kalari, L. Wang, W. S. Harmsen, J. Yuan, J. C. Boughey, M. P. Goetz, J. N. Sarkaria, Z. Lou, and R. W. Mutter, "ATR Inhibition Is a Promising Radiosensitizing Strategy for Triple-Negative Breast Cancer," Mol. Cancer Ther. 17 no. 11, (Nov, 2018) 2462-2472. 104

[201] S. Hamis, A. Yaromina, L. Dubois, M. Kohandel, P. Lambin, and G. G. Powathil, "Combining Hypoxia-Activated Prodrugs and Radiotherapy in silico: Impacts of Treatment Scheduling and the Intra-Tumoural Oxygen Landscape," (To Appear) . 110, 111

[202] F. P. Vendetti, A. Lau, S. Schamus, T. P. Conrads, M. J. O’Connor, and C. J. Bakkenist, "The orally active and bioavailable ATR kinase inhibitor AZD6738 potentiates the anti-tumor effects of cisplatin to resolve ATM-deficient non-small cell lung cancer in vivo," Oncotarget 6 no. 42, (Dec, 2015) 44289-44305. 112

[203] S. A. Visser, D. P. de Alwis, T. Kerbusch, J. A. Stone, and S. R. Allerheiligen, "Implementation of quantitative and systems pharmacology in large pharma," CPT Pharmacometrics Syst Pharmacol 3 (Oct, 2014) e142. 114

[204] S. Hamis, S. Stratiev, and G. Powathil, "Uncertainty and sensitivity analyses methods for agent-based models: A review," (To Appear) . 114, 157

[205] MATLAB, version 1.8.0_202 (R2019n). The MathWorks Inc., Natick, Massachusetts, 2019. 115, 147

[206] J. Metzcar, Y. Wang, R. Heiland, and P. Macklin, "A Review of Cell-Based Computational Modeling in Cancer Biology," JCO Clin Cancer Inform 3 (02, 2019) 1-13. 125

[207] M. Read, P. S. Andrews, J. Timmis, and V. Kumar, "Techniques for grounding agent-based simulations in the real domain: a case study in experimental 


\section{REFERENCES}

autoimmune encephalomyelitis," Mathematical and Computer Modelling of Dynamical Systems 18 no. 1, (2012) 67-86. 128, 129

[208] S. Lin, W. Li, X. Qian, P. Ma, and M. Yang, "A simulation model validation and calibration platform," pp. 687-693. 12, 2018. 128

[209] J. L. Gevertz and J. R. Wares, "Developing a Minimally Structured Mathematical Model of Cancer Treatment with Oncolytic Viruses and Dendritic Cell Injections," Comput Math Methods Med 2018 (2018) 8760371. 128

[210] S. Blower and H. Dowlatabadi, "Sensitivity and uncertainty analysis of complex models of disease transmission: An hiv model, as an example," International Statistical Review $62(08,1994), 128$

[211] A. Charzyńska, A. Nałęcz, M. Rybiński, and A. Gambin, "Sensitivity analysis of mathematical models of signaling pathways," BioTechnologia. 93 (3) (2012) 291-308. 129, 142

[212] K. O. McGraw and S. P. Wong, " A common language effect size statistic.," Psychological Bulletin 111 no. 2, (1992) 361-365. 130, 136

[213] A. Vargha and H. D. Delaney, "A Critique and Improvement of the CL Common Language Effect Size Statistics of McGraw and Wong.," Journal of Educational and Behavioral Statistics 25(2) (2000) 101âĂŞ132. 130, 131, 133, 134,135

[214] J. Cohen, "The statistical power of abnormal-social psychological research: a review," J Abnorm Soc Psychol 65 (Sep, 1962) 145-153. 135

[215] O. Cohen, "Statistical Power Analysis for the Behavioral Sciences (Second Edition) ," Lawrence Erlbaum Associates (1988) . 135, 136

[216] J. Ruscio and T. Mullen, "Confidence Intervals for the Probability of Superiority Effect Size Measure and the Area Under a Receiver Operating Characteristic Curve," Multivariate Behavioral Research 47 no. 2, (2012) 201-223. 135

[217] M. D. McKay, R. J. Beckman, and W. J. Conover, "Comparison of three methods for selecting values of input variables in the analysis of output from a computer code," Technometrics 21 no. 2, (1979) 239-245. 145 
[218] M. D. McKay, "Latin hypercube sampling as a tool in uncertainty analysis of computer models," in Proceedings of the 24th Conference on Winter Simulation, WSC '92, pp. 557-564. ACM, New York, NY, USA, 1992. http://doi.acm.org/10.1145/167293.167637. 145

[219] R. Sheikholeslami and S. Razavi, "Progressive latin hypercube sampling: An efficient approach for robust sampling-based analysis of environmental models," Environmental Modelling Software $\mathbf{9 3}(07,2017)$ 109-126, 145, 146, 147

[220] G. Manache and C. Melching, "Sensitivity of Latin Hypercube Sampling to sample size and distributional assumptions," 07, 2007. 147

[221] R. Iman and J. Helton, "Comparison of uncertainty and sensitivity analysis techniques for computer models," Report NUREGICR-3904, SAND 84-1461, Sandia National Laboratories, Albuquerque, New Mexico $(3,1985)$. 147

[222] M. M. Mukaka, "Statistics corner: A guide to appropriate use of correlation coefficient in medical research," Malawi Med J 24 no. 3, (Sep, 2012) 69-71. 149. 150

[223] P. Schober, C. Boer, and L. A. Schwarte, "Correlation Coefficients: Appropriate Use and Interpretation," Anesth. Analg. 126 no. 5, (05, 2018) 1763-1768. 150

[224] T. Krehbiel, "Correlation coefficient rule of thumb," Decision Sciences Journal of Innovative Education 2 (01, 2004) 97-100, 150

[225] M. F. McGuire, H. Enderling, D. I. Wallace, J. Batra, M. Jordan, S. Kumar, J. C. Panetta, and E. Pasquier, "Formalizing an integrative, multidisciplinary cancer therapy discovery workflow," Cancer Res. 73 no. 20, (Oct, 2013) 6111-6117. 174 